Kathrin Dengler, Heiner Fangerau (Hg.)

\title{
Zuteilungskriterien im Gesundheitswesen: Grenzen und Alternativen
}

Eine Einführung mit medizinethischen und philosophischen Verortungen 
Kathrin Dengler, Heiner Fangerau (Hg.)

Zuteilungskriterien im Gesundheitswesen:

Grenzen und Alternativen 

Kathrin Dengler, Heiner Fangerau (Hg.)

\section{Zuteilungskriterien im Gesundheitswesen: Grenzen und Alternativen}

Eine Einführung mit medizinethischen

und philosophischen Verortungen

[transcript $]$ 
Entstanden im Rahmen eines Kooperationsprojektes mit Lilly Deutschland

\section{(6) $(1) \Theta$}

Dieses Werk ist lizenziert unter der Creative Commons Attribution-NonCommercial-NoDerivs 4.0 Lizenz (BY-NC-ND). Diese Lizenz erlaubt die private Nutzung, gestattet aber keine Bearbeitung und keine kommerzielle Nutzung. Weitere Informationen finden Sie unter https://creativecommons.org/licenses/by-nc-nd/4.o/deed.de/. Um Genehmigungen für Adaptionen, Übersetzungen, Derivate oder Wiederverwendung zu kommerziellen Zwecken einzuholen, wenden Sie sich bitte an rights@transcript-verlag.de

\section{(C) 2013 transcript Verlag, Bielefeld}

Die Verwertung der Texte und Bilder ist ohne Zustimmung des Verlages urheberrechtswidrig und strafbar. Das gilt auch für Vervielfältigungen, Übersetzungen, Mikroverfilmungen und für die Verarbeitung mit elektronischen Systemen.

\section{Bibliografische Information der Deutschen Nationalbibliothek}

Die Deutsche Nationalbibliothek verzeichnet diese Publikation in der Deutschen Nationalbibliografie; detaillierte bibliografische Daten sind im Internet über http://dnb.d-nb.de abrufbar.

Umschlagkonzept: Kordula Röckenhaus, Bielefeld

Satz: Mark-Sebastian Schneider, Bielefeld

Druck: Majuskel Medienproduktion GmbH, Wetzlar

Print-ISBN 978-3-8376-2290-4

PDF-ISBN 978-3-8394-2290-8

Gedruckt auf alterungsbeständigem Papier mit chlorfrei gebleichtem Zellstoff.

Besuchen Sie uns im Internet: http://www.transcript-verlag.de

Bitte fordern Sie unser Gesamtverzeichnis und andere Broschüren an unter: info@transcript-verlag.de 


\section{Inhalt}

Die Bewertung von Gesundheitsleistungen

Einführende Überlegungen

Heiner Fangerau und Kathrin Dengler $\mid 7$

\section{Die Bewertung von Gesundheitsleistungen}

Zentrale gesundheitsökonomische Bewertungsverfahren

Ein einführender Überblick in Anlehnung an Schöffski

und von der Schulenburg

Kathrin Dengler | 27

Beschreibungsdimensionen für

gesundheitsökonomische Bewertungsverfahren

Eine einführende Diskussion von Zielgrößen, Implikationen und ethischen Problemfeldern

Kathrin Dengler und Heiner Fangerau $\mid 51$

\section{Gesundheit und Gerechtigkeit}

Konzeptionen von Gerechtigkeit

und der Umgang mit begrenzten Ressourcen

Christian Lenk | 77

Prinzipien einer gerechten Rationierung

im Gesundheitswesen

Walter Pfannkuche | 113 
Anerkennung des Individuums oder des Kollektivs?

Sozialhygienische Zuteilungskriterien im Gesundheitswesen

bei Alfred Grotjahn, Max Mosse und Gustav Tugendreich im

Vergleich zur heutigen Sozialepidemiologie

Sebastian Kessler | 139

\section{Werthaltungen UND IHRE KRITISChe ValidierUNG}

Konzeptionelle und methodische Probleme der

Lebensqualitätsmessung als Grundlage der Bewertung der

Ergebnisse medizinischer Maßnahmen

Reinhold Kilian | 171

Wert und Werthaltungsbegründung

Zur Vereinbarkeit von moralischen, ökonomischen, rechtlichen und medizinischen Werten

Günter Fröhlich | 195

Ressourcenzuteilung im Gesundheitswesen

Zur Logik der Leistungssteigerung und

Effizienzmaximierung und ihren ethischen Grenzen

Arne Manzeschke | 223

Autorenverzeichnis | 247

Register | 249 


\section{Die Bewertung von Gesundheitsleistungen}

Einführende Überlegungen

Heiner Fangerau und Kathrin Dengler ${ }^{1}$

\section{Die Bewertung von Gesundheitsleistungen}

Medizinische Gesundheitsleistungen zielen auf eine Wiederherstellung, Aufrechterhaltung oder Besserung der Gesundheit. Es existieren unzählige Angebote für Gesundheitsleistungen und selbst in einem konzeptuell relativ geschlossenen Rahmen dessen, was als Gesundheit verstanden wird und wie diese herzustellen sei, können widersprüchliche Bewertungen einer medizinischen Leistung koexistieren. Die akademische Medizin (Universitätsmedizin) des 19., 20. und frühen 21. Jahrhunderts stellt keine Ausnahme dar. Sie bietet zahlreiche Beispiele für unterschiedliche Evaluationen der medizinischen Güte einzelner therapeutischer Verfahren. Verwiesen sei hier nur exemplarisch auf den seit mindestens 200 Jahren währenden innermedizinischen Streit über die Frage, ob es Beweise (heute: evidence) für prophylaktische Effekte der männlichen Zirkumzision gebe (vgl. Task Force on Circumcision 2012, Gollaher 1994) oder auf den noch ungeklärten Disput, ob im Falle einer Blinddarmentzündung eine Appendektomie oder eine konservative Behandlung die besseren Ergebnisse bringe (Simillis et al. 2009).

1 | Wir danken Uta Bittner und Christian Lenk für ihre kritischen Anmerkungen, Kommentare und Korrekturen. 
Noch komplizierter wird die Bewertung von Gesundheitsleistungen, wenn nicht nur die Frage nach der medizinischen Wertigkeit einer Behandlung im Raum steht, sondern mehrdimensional auch noch die Kosten einer Therapie in die Überlegungen mit einbezogen werden sollen oder Kosten-Nutzen Evaluationen zum Vergleich verschiedener Verfahren angestrebt werden.

Multidimensionale Bewertungen der Versuche, Gesundheit herzustellen oder zu bewahren, sind keineswegs ein neues Phänomen. Für das 19. Jahrhundert etwa haben Alfons Labisch und Reinhard Spree im von ihnen herausgegebenen »Krankenhaus-Report 19. Jahrhundert« eindrücklich gezeigt, wie vielfältig Kosten- und Nutzen-Aspekte im Zusammenhang mit dem spezifischen Leistungsanbieter Krankenhaus diskutiert wurden (Labisch, Spree 2001). Allerdings scheint sich zumindest die politische Debatte um die Bewertung von Gesundheitsleistungen seit dem Ende der 1970er Jahre in der Bundesrepublik Deutschland mit dem ersten Kostendämpfungsgesetz von 1977 beschleunigt zu haben (»Krankenversicherungs-Kostendämpfungsgesetz vom 27. Juni 1977« [BGBl. I S. 1069]; vgl. auch Ritter 1998). Angesichts steigender Kosten im Gesundheitswesen hatte zum Beispiel die Bundesregierung unter Gerhard Schröder 2004 das »Gesetz zur Modernisierung der gesetzlichen Krankenversicherung « erlassen, das mit der Zielsetzung antrat, die Kosten-Nutzen Relation der Gesundheitsversorgung in Deutschland zu verbessern (»Gesetz zur Modernisierung der gesetzlichen Krankenversicherung « vom 14. November 2003 [BGBl. I S. 2190]). Derzeit wiederum beschäftigen sich unterschiedlichste Akteure aus verschiedenen Motivationen mit der Thematik. Neben dem 2004 im Rahmen des genannten Gesetzes geschaffenen Institut für Qualität und Wirtschaftlichkeit im Gesundheitswesen (IQWiG) widmen sich Ministerien, die Pharmazeutische Industrie, Krankenkassen- und Versicherungen sowie Krankenhausverwalter, Ökonomen und Ärzte dem Problemfeld der Bewertung von Gesundheitsleistungen.

Ausgehend von zahlreichen Vorüberlegungen und einschlägigen Arbeiten zu den Methoden der Bewertung, ihren Zielpara- 
metern und ihren Problemen ${ }^{2}$ möchten wir mit diesem Band eine kurze Einführung in die Debatte geben und verfolgen dabei einen multiperspektivischen Ansatz. So werden die ethischen Implikationen einer solchen Evaluation thematisiert, indem eine Berücksichtigung der theoretischen und praktischen moralischen Folgerungen erfolgt, die sich aus der Anwendung des einen oder anderen etablierten Messinstrumentes ergeben. Da die Frage nach Gesundheit, Krankheit und Gesundheitsversorgung eine Vielzahl von ethischen Erwägungen aufwirft, stellen die beteiligten Autoren verschiedene philosophische, gerechtigkeitstheoretische, ethische, medizinhistorische sowie gesundheitsökonomische Herangehensweisen vor, die sich diesem Themenkomplex widmen.

Zur Illustration sei nur auf den im Folgenden skizzierten Einfluss des Gesundheitsverständnisses auf die Beurteilung eines möglichen ethischen Anrechts auf Gesundheit verwiesen, eine Beurteilung, die wiederum zurückwirkt auf die Frage, welches Instrument zur Kosten-Nutzen-Messung dem gesundheitsbezogenen Problemkreis überhaupt angemessen ist. Die dabei immer durchschimmernde Grundfragestellung betrifft den Wert von >Gesundheit $<$ - sowohl in individueller als auch in gesellschaftlicher Dimension.

\section{Herausforderungen maximalistischer GESUNDHEITSDEFINITIONEN}

So sehr sich alles medizinische Handeln auf die Gesundheit bezieht, so sehr ist der Grundbegriff >Gesundheit< durch eine hohe Variabilität geprägt: Gesundheit wird in diachroner und synchroner Perspektive vor dem Hintergrund jeweiliger kulturtheoretisch er-

2 | Vgl. exemplarisch Herder-Dornreich 1994; Sloan 1995; Rychlik 1999; Kielhorn und Schulenburg 2000; Marckmann, Liening, Wiesing 2003; Schmidt-Wilke 2004; Drummond et al. 2005; Fleßa 2005; Schöffski und Schulenburg 2008; Deutscher Ethikrat 2012. 
fassbarer Bezugsrahmen verschiedentlich definiert (vgl. u.a. Schäfer et al. 2008). Ärztliche Konzepte von Krankheit und Gesundheit dienen dabei nach Rothschuh der Ordnung von Krankheitssymptomen, der Erklärung der Entstehung von Krankheiten und der Rechtfertigung ärztlicher Handlungen (Rothschuh 1978). Die Ordnung der Erkrankungen bringt wiederum das Problem mit sich, Diagnosegrenzen und den Unterschied zwischen Gesundheit und Krankheit zu definieren. Hier lassen sich im historischen Verlauf einige Modelle unterscheiden, nach denen Krankheiten entweder als klassifizierbare Entitäten begriffen werden, die bipolar von Gesundheit abgegrenzt werden können oder nach denen Krankheit und Gesundheit die Pole eines Kontinuums bilden, auf dem die Grenzen fließend sind (vgl. überblicksweise und für weiterführende Literatur: Fangerau und Martin 2011).

Eine bekannte, sehr prägende und weitreichende Gesundheitsdefinition offeriert die Weltgesundheitsorganisation (WHO). Sie expliziert ein maximalistisches Grundverständnis am Extrempol dieses Kontinuums, wonach Gesundheit als »Zustand vollkommenen körperlichen, seelischen und sozialen Wohlbefindens « (WHO Präambel 1946) verstanden wird (vgl. Lenk 2011). Diese Definition bringt es mit sich, dass sich im gesellschaftlichen Diskurs auch der Krankheitsbegriff bzw. das Gesundheitsverständnis weniger am Fehlen von Krankheit als an einer Orientierung an einem möglichen Optimum an Gesundheit festmachen lässt. Thomas Lemke und Regine Kollek beschreiben mit Blick auf die jüngsten Entwicklungen diesen Gesundheitsdiskurs treffend, indem sie festhalten:

"Wurde Gesundheit in der Vergangenheit meist als ein Zustand bestimmt, in dem man über das eigene (Wohl-)Befinden nicht nachdachte, hat diese negative Vorstellung von Gesundheit als Abwesenheit von Krankheit in den letzten Jahrzehnten zunehmend an Überzeugungskraft verloren. An die Stelle einer passiven Konzeption von Gesundheit trat eine Deutung, die Gesundheit vor allem als aktives Sichwohlfühlen begreift und weniger von einem statischen Gesundheits-Zustand als von einem dynamischen - und 
prinzipiell unabschließbaren - Prozess des Immer-Gesünder-Werdens ausgeht" (Lemke und Kollek 2011, S. 171).

Dieser weitreichende Ansatz impliziert, dass das >Projekt Gesundheit< theoretisch sowohl für das Individuum als auch für die Gesellschaft nie abgeschlossen sein wird. Das Prinzip der fortlaufenden Steigerbarkeit von Gesundheit ist Kernbestandteil in solchen Konzeptionen (Lemke und Kollek 2011, S. 173). Entscheidend für eine Betrachtung der Bemessung von Gesundheitsleistungen ist in diesem Kontext, dass der Gesundheit nicht nur ein besonderer (Lebens-)Wert, sondern vielmehr ein Status als ein »besonderes Gut« (Gosepath 2007, S. 20) zugewiesen wird.

Gesundheit ist demnach kein Gut im Sinne eines Konsumgutes, denn die Nutzung von Gesundheitsleistungen kann nicht beliebig weit hinaus- bzw. aufgeschoben werden (vgl. Rosentreter 2011). Auch haben Individuen kaum die Möglichkeit, sich Gesundheit in toto zu erkaufen. Gleichzeitig besteht aber ein elementares Bedürfnis des Menschen in dem Bestreben, gesund zu sein. Gesundheit ist nach Autoren wie zum Beispiel Norman Daniels (vgl. Daniels 1985) die Ermöglichungsbedingung für die Verfolgung und potentielle Erreichung weiterer Lebensziele und -entwürfe. Daher wird Gesundheit mitunter auch als konditionales Gut bezeichnet - ähnlich wie Frieden, Sicherheit oder Freiheit. Konditional bedeutet in diesem Fall, dass diese Güter »die notwendige Bedingung aller Lebenspläne bilden.« (Gosepath 2007, S. 20) Daraus resultiert auch das Unbehagen, das mit Blick auf Versuche entsteht, Gesundheitszustände in Geldwerte umzuwandeln, um so eine einheitliche Bemessungsgrundlage zu schaffen (vgl. u.a. für ein historisches Beispiel Fangerau 2009 und die dort zitierte Literatur).

Gesundheit ist aber nicht nur ein besonderes, konditionales Gut, sondern auch ein öffentliches Gut. Denn von der Gesundheit anderer profitiert jeder einzelne (z.B. bei Infektionskrankheiten wegen einer geringeren Ansteckungsgefahr, wenn alle anderen Mitmenschen gesund sind). Zudem gilt: »Die Gesundheit der Gesellschaft ist zugleich die Bedingung der Möglichkeit für die Produktionskraft 
eines Landes « (Gosepath 2007, S. 21). Wenn folglich Gesundheit als notwendige (wenngleich auch nicht immer hinreichende) Voraussetzung vieler individueller wie gesellschaftlicher Lebensentwürfe betrachtet wird, dann mag es nicht verwundern, dass mit einem Zur-Verfügung-Stellen von Gesundheitsleistungen auch ein Mindestmaß an Chancengleichheit assoziiert wird. Im SGB V spiegelt sich beispielsweise diese Assoziation darin wider, dass Gesundheitsleistungen Patientennutzen-Parameter unterstützen sollen, die Ermöglichungsbedingungen im Blick haben. Im Kontext der Bewertung von Arzneimitteln (SGB V, $\$ 35$ b Kosten-Nutzen-Bewertung von Arzneimitteln) gelten zum Beispiel folgende Ziele:

"Beim Patienten-Nutzen sollen insbesondere die Verbesserung des Gesundheitszustandes, eine Verkürzung der Krankheitsdauer, eine Verlängerung der Lebensdauer, eine Verringerung der Nebenwirkungen sowie eine Verbesserung der Lebensqualität, bei der wirtschaftlichen Bewertung auch die Angemessenheit und Zumutbarkeit einer Kostenübernahme durch die Versichertengemeinschaft, angemessen berücksichtigt werden“ (SGB V $\S 35 b)$.

Das Problem des oben skizzierten, neuartigen maximalistischen Gesundheitsverständnisses liegt nun zum einen darin, dass ein solches in seinem Status als Ermöglichungsbedingung nicht unumstritten ist, da eventuell auch ein weniger maximaler Ansatz ausreicht, um gesellschaftlich und individuell $\mathrm{zu}$ »funktionieren «, zum zweiten sind die Kosten eines Maximalstrebens sehr hoch, zum dritten teilen Individuen und Gesellschaft nicht notwendigerweise die gleichen Zielparameter von Gesundheit. In die Definition von Gesundheit und Krankheit fließen stets sowohl objektive (medizinisch-diagnostische), subjektive (persönliche, individuelle) als auch inter-subjektive Elemente (Anerkennung von Krankheit) ein (vgl. Paul 2006). Unterschiedliche Zuschreibungen von Krankheit und Gesundheit wirken sich folglich auch auf den Umgang mit Krankheit und Gesundheit sowie die Vorstellung und Anerkennung von Behandlungsansprüchen zur Zielerreichung von Gesundheit aus. 
Hier schließen sich die beiden Fragen an, inwiefern es ein Recht bzw. einen (legitimen) Anspruch auf Gesundheitsleistungen gibt bzw. überhaupt geben kann und wie angesichts eines maximalen Gesundheitsverständnisses die Bewertung einzelner Leistungen Einfluss auf ihre Anrechenbarkeit hat.

\section{Anrecht auf Gesundheitsleistungen?}

Während es ein Recht auf Gesundheit nicht geben, da diese nicht garantiert werden kann, so ergibt sich doch eine rechtliche und moralische Begründung für ein Anrecht auf Gesundheitsleistungen schon aus seiner Formulierung als ein Menschenrecht in Artikel 25 der UN-Resolution zur Erklärung der Menschenrechte (Resolution 217 A [III] der Generalversammlung vom 10. Dezember 1948). ${ }^{3}$ Dennoch ist diese Frage nach diesem Anrecht damit nicht erledigt, denn sowohl sein Geltungsbereich als auch der Umfang der medizinischen Leistungen, auf die dieses Anrecht bestehen soll, sind diskussionsbedürftig.

Die grundsätzliche Begründung für ein Anrecht auf medizinische Leistungen kann dabei intrinsisch in der Würde des Menschen liegen, auf deren Basis zum Beispiel Schmerzen zu vermeiden sind und Menschen, die unter Schmerz leiden, zu helfen ist (Reiter-Theil et al. 2008). Auch können extrinsisch der Respekt vor anderen oder Regeln der Barmherzigkeit ein solches Anrecht evozieren. Anders

3 | In der deutschen Fassung ist von "ärztlicher Versorgung" die Rede, in der englischen Fassung von "medical care". "1. Jeder hat das Recht auf einen Lebensstandard, der seine und seiner Familie Gesundheit und Wohl gewährleistet, einschließlich Nahrung, Kleidung, Wohnung, ärztliche Versorgung und notwendige soziale Leistungen, sowie das Recht auf Sicherheit im Falle von Arbeitslosigkeit, Krankheit, Invalidität oder Verwitwung, im Alter sowie bei anderweitigem Verlust seiner Unterhaltsmittel durch unverschuldete Umstände." www.ohchr.org/en/udhr/pages/language.aspx? langid=ger (zuletzt geprüft am 08.03.2013) 
als die völkerrechtlich und grundgesetzlich verbriefte Achtung der Würde lassen sich Respekt und Barmherzigkeit aber nicht im Sinne eines Rechts einfordern. Ein geeigneter Ansatz, ein Anrecht auf medizinische Leistungen herzuleiten, scheint vor allem in der Gerechtigkeitsphilosophie und in ihrem Bezug zum Gesellschaftsvertrag zu finden zu sein, die beide in bestimmten Teilen auf menschliche Würde als Ausgangspunkt rekurrieren (vgl. Lenk 2010 und den Aufsatz Lenk in diesem Band).

Der Gesellschaftsvertrag als philosophische Argumentationsfigur zielt einerseits darauf ab, durch Kooperation Vorteile zu erreichen. Andererseits sichert er Rechte und Grundprinzipien zu, zu denen auch die schon oben angesprochene generelle Chancengleichheit zählt. Zur Wahrung dieser Chancengleichheit gehört nach Ansicht verschiedener Gerechtigkeitstheoretiker auch, dass gewisse Grundgüter gerecht verteilt sein sollten oder dass zumindest auf einen Ausgleich hingearbeitet werden sollte (vgl. Lenk 2010). Grundgüter können dabei sowohl materielle Güter als auch soziale Rechte sein. Wird Gesundheit wie oben beschrieben als »konditionales Gut« verstanden, das zu den basalen Bedürfnissen (Gosepath 2004) gehört, die die Befriedigung anderer Bedürfnisse erst ermöglichen, so gehört auch die Versorgung mit medizinischen Leistungen zu diesen Grundgütern.

Zentral für die Frage des möglichen Anrechts auf Gesundheitsleistungen erscheinen hierbei verschiedene Gerechtigkeitsprinzipien: so z.B. das Prinzip der ausgleichenden Gerechtigkeit im Zusammenhang mit dem Ausgleich natürlicher Ungleichheit (z.B. genetische Prädispositionen, Behinderungen), das Bedarfsprinzip, das die Verteilung von beispielsweise Leistungen am Bedarf bemisst (z.B. nach Unfällen, tödliche Krankheiten) und ein auf Chancengleichheit zielendes Prinzip, das gleiche (Start-)Möglichkeiten für alle Beteiligten zur Erreichung möglicher Ziele schaffen möchte (z.B. in der Geburtshilfe und Kinderheilkunde) (vgl. den Beitrag von Christian Lenk in diesem Band).

Im Sinne dieser Überlegungen wurde die Chancengleichheit von Norman Daniels in seinem viel zitierten Buch Just Health Care 
herangezogen, um ein mögliches Anrecht auf Gesundheitsleistungen zu begründen. In Anlehnung an John Rawls (Rawls 1999) und das von ihm vertretene Konzept der Fairness diskutiert Daniels das Problem der gerechten Verteilung medizinischer Leistungen in modernen Gesellschaften (Daniels 1985). Auch er sieht die Gesundheit als Grundbedingung für Chancengleichheit in der Gesellschaft. Gesundheit ermöglicht erst gesellschaftliche Teilhabe und die Verfolgung eigener Ziele. Damit wendet sich Daniels auch gegen Positionen, die Krankheit und Gesundheit im Bereich persönlichen Unglücks, der unabänderlichen natürlichen Voraussetzungen mit ihren naturgegebenen Ungleichheiten oder im Umfeld des rein Privaten verorten und fordert die Solidarität einer Gemeinschaft zur Wahrung oder Herstellung dieser Chancengleichheit ein.

Wenn sich aber auf diese Weise in einer sozialen Gemeinschaft ein Anrecht auf medizinische Leistungen begründen lässt, um möglichst große Chancengleichheit zu schaffen, so muss sowohl das Leiden bzw. die Krankheit als auch die medizinische Leistung selbst gesellschaftlich anerkannt sein. Nur so lässt sich zum Beispiel in juristischer Lesart ein Leistungsrecht (auf Gesundheitsleistungen) einfordern, das eventuell mit den Freiheitsrechten anderer kollidiert, indem es diesen zum Beispiel Solidaritätspflichten auferlegt (vgl. Seelmann 2008, S. 185). Das bedeutet, dass ein gesellschaftlicher Konsens darüber herrschen muss, welche Krankheit Anerkennung als die Chancengleichheit mindernde Beeinträchtigung findet und welche wie bewertete medizinische Leistung als Behandlung anerkannt ist (vgl. die Übersicht von Fischer 2013), womit sich der Kreis zu den obigen Ausführungen zu Gesundheitskonzeptionen wieder schließt. Verschiedene Einflüsse wirken dabei auf die mögliche Anerkennung einer Krankheit (aus sozial-gesellschaftlicher Sicht) und die Anerkennung einer entsprechenden medizinischen Kompensation. Empirisch lässt sich zum Beispiel der Einfluss von Überlegungen zur Schwere einer Erkrankung oder einer möglichen Schuldzuweisung auf die Anerkennung eines Anrechts auf medizinische Leistungen erheben. So konnten beispielsweise Georg Schomerus et al. in Bezug auf psychiatrische Erkrankungen zeigen, dass 
die Attribution einer angeblichen eigenen Verantwortung für ein Leiden, der Wunsch nach sozialer Distanz zu einem bestimmten Störungsbild und das Fehlen einer nachweislich effektiven Therapie dazu führen, dass Befragte angeben, für diese Erkrankungen Leistungen eher kürzen zu wollen (Schomerus 2006).

Wenn eine Gesellschaft also auf Basis von Gerechtigkeitserwägungen den Schluss zieht, dass sie ein Anrecht auf Gesundheitsleistungen anerkennt, so muss sie dennoch in eine Diskussion treten, welche Leistungen zur Behandlung welcher Erkrankungen sie anzuerkennen bereit ist. Letztendlich führt nur ein transparenter, im politischen Diskurs kommunizierter, juristisch verbriefter Katalog zur im Alltag umsetzbaren Anerkennung eines Anrechts auf bestimmte Leistungen. Die bei diesen Erwägungen heranzuziehenden Kriterien können nicht allein ökonomische sein. Medizinische Gesichtspunkte sind ebenso einzubeziehen (vgl. Porzsolt et al. 2010) wie ethische Erwägungen. Nicht zuletzt sind hier Fragen der individuellen wie gesellschaftlichen Präferenzen und Werthaltungen genauso zu berücksichtigen wie theoretische Überlegungen zur Begründungsfähigkeit von interpersonalen Ansprüchen und Erwartungshaltungen.

\section{Aufbau und Struktur des Buches ${ }^{4}$}

Ein solcher ganzheitlicher Ansatz ist anfällig für disziplinäre Bruchlinien und für an diesen auftretende potentielle ethische Problemfelder, die sich allein schon aus unterschiedlichen Blickwinkeln auf das Problem der Bewertung von Gesundheitsleistungen ergeben. Eine erste Orientierung über unterschiedliche Perspektiven auf

4 | Wir wollen an dieser Stelle darauf hinweisen, dass sich nicht alle in den Beiträgen vertretenen Positionen mit den Ansichten der Herausgeber decken. Aus Gründen einer ergebnisoffenen Auseinandersetzung versprechen wir uns allerdings mit dieser Herangehensweise eine möglichst umfassende und vielschichtige Debatte. 
das Grundphänomen der gerechten Bewertung von Gesundheitsleistungen zu bieten, ist das Ziel dieses Bandes. Es sollen sowohl philosophische Fragen der Fairness und Gerechtigkeit, der Bedürftigkeit und der ethischen Legitimation von Anspruchshaltungen behandelt, als auch allgemeingesellschaftliche Fragen der Wertzuschreibung sowie der gesundheitsökonomischen und medizinischen Messung von Lebensqualität im Kontext von Krankheit und Gesundheit vorgestellt werden.

$\mathrm{Zu}$ diesem Zweck wird im vorliegenden Band das mehrdimensionale Spannungsverhältnis zwischen (gesundheits)ökonomischem Methodenrepertoire zur Bewertung von Gesundheitsleistungen, gerechtigkeitstheoretischen Modellen der fairen Verteilung von Ressourcen sowie nutzenorientierten Verteilungsansätzen aus individueller und gesellschaftlicher Perspektive an exemplarischen Problemfeldern und Herausforderungen diskutiert. Der erste Abschnitt »Die Bewertung von Gesundheitsleistungen « nimmt zunächst die etablierten Methoden zur Evaluation von Gesundheitsleistungen in den Blick. Kathrin Dengler stellt in ihrem diesen Themenblock einführenden Überblicksartikel »Zentrale gesundheitsökonomische Bewertungsverfahren - Ein einführender Überblick in Anlehnung an Schöffski und von der Schulenburg« die verschiedenen, der ökonomischen Disziplin entlehnten Evaluationsverfahren vor, die von der Kosten-Analyse bis hin zur Kosten-Nutzwert-Analyse reichen. Dabei orientiert sie sich eng an den Ausführungen von Schöffski und Graf von der Schulenburg, die mit ihrem Band »Gesundheitsökonomische Evaluationen« (Schöffski und Schulenburg 2008) ein detailreiches Übersichtswerk vorgelegt haben. Dengler geht in ihren Ausführungen auf die unterschiedlichen Zielgrößen ausgewählter Berechnungsverfahren ein und expliziert überblicksartig die modellinhärenten Spezifika der einzelnen Bewertungsansätze.

An diese einführende Übersicht schließt sich der analytische Beitrag »Beschreibungsdimensionen für gesundheitsökonomische Bewertungsverfahren - Eine einführende Diskussion von Zielgrößen, Implikationen und ethischen Problemfeldern« von Kathrin 
Dengler und Heiner Fangerau an, der verschiedene Beschreibungsdimensionen für die Auseinandersetzung mit Bewertungsmethoden vorstellt. Dabei wird unter anderem auch die Stellungnahme des Deutschen Ethikrates mit dem Titel »Nutzen und Kosten im Gesundheitswesen - Zur normativen Funktion ihrer Bewertung « aufgegriffen.

Der zweite Abschnitt »Gesundheit und Gerechtigkeit« vertieft die oben angerissenen Überlegungen und wird durch den Beitrag »Konzeptionen von Gerechtigkeit und der Umgang mit begrenzten Ressourcen« von Christian Lenk eingeleitet. Lenk geht auf Basis von verschiedenen, in einem Konkurrenzverhältnis stehenden Ansätzen aus der Ethik und der politischen Philosophie der Frage nach, welche Optionen zur »Lösung konkreter Verteilungsprobleme« unter der Bedingung knapper Ressourcen denkbar sind.

Den Blick auf allgemeine Fragen der Verteilungsgerechtigkeit richtet danach Walter Pfannkuche in seinem Beitrag »Prinzipien einer gerechten Rationierung im Gesundheitswesen«. Seiner Ansicht nach ist das Problem der gerechten Versorgung im Gesundheitswesen eng an die Frage nach einer gerechten Verteilung der Einkommen innerhalb einer Gesellschaft geknüpft. Indem er für letztere eine Lösung entwirft, zeigt er zugleich die Mehrdimensionalität des zugrundeliegenden Problems auf.

Zum Abschluss dieses Abschnitts geht Sebastian Kessler in seinem historisch-analytischen Beitrag »Anerkennung des Individuums oder des Kollektivs? Sozialhygienische Zuteilungskriterien im Gesundheitswesen bei Alfred Grotjahn, Max Mosse und Gustav Tugendreich im Vergleich zur heutigen Sozialepidemiologie« der Frage nach, wie zu Beginn der Sozialepidemiologie die Anerkennung von Gesundheits- und Krankheitszuständen begründet wurde. Er expliziert am Beispiel von Alfred Grotjahns Schriften sowie dem Werk von Max Mosse und Gustav Tugendreich, welche Zuteilungskriterien für Anerkennung in Ansatz gebracht wurden, wie auf der Basis von solchen Zuteilungskriterien gesundheitspolitisch motivierte Zwangsmaßnahmen mit Verweis auf Krankheit legitimiert 
wurden und wie diese Bezugnahmen sich im Zeitverlauf verändert haben.

Im dritten Abschnitt »Werthaltungen und ihre kritische Validierung«werden zuletzt individuelle und gesellschaftliche Präferenzen in Bezug auf Gesundheit und ihre Bewertung betrachtet. Reinhold Kilian zeigt in seinem Beitrag »Konzeptionelle und methodische Probleme der Lebensqualitätsmessung als Grundlage der Bewertung der Ergebnisse medizinischer Maßnahmen« die Leistungsfähigkeit - aber auch die Grenzen - des Konzeptes der Messung von Lebensqualität auf. Bezugnehmend auf verschiedene Vorstellungen vom >guten Leben zeichnet Kilian die Entwicklung der Idee zur Messung von Lebensqualität nach und verweist darauf, dass der Ansatz der Lebensqualitätsmessung ein verhältnismäßig junges Phänomen darstellt, das erst Mitte des vorigen Jahrhunderts entfaltet wurde.

In seinem Beitrag »Wert und Werthaltungsbegründung. Zur Vereinbarkeit von moralischen, ökonomischen, rechtlichen und medizinischen Werten « geht Günter Fröhlich darauf der grundsätzlichen der Frage nach, was unter Werten zu verstehen ist, wo der begriffliche Ursprung von Werten und Werttheorien liegt und wie unterschiedliche Werte zueinander in Verbindung gebracht werden können. Fröhlich zeigt gleichfalls auf, wie der konsequentialistische Ethikansatz in Form des Utilitarismus in der Debatte um gesundheitliche Verteilungs- und Bewertungsfragen verteidigt werden kann und wendet sich dabei gegen häufig vorgebrachte Einwände, wobei er darauf hinweist, dass sich die drei Ethikbegründungstypen - Utilitarismus, Deontologie, Tugendethik - nicht in solch gravierendem Maße voneinander unterscheiden, wie es oft in der Debatte um die Bewertung von Gesundheitsleistungen dargestellt wird.

Im darauffolgenden, den Band abschließenden Beitrag »Ressourcenzuteilung im Gesundheitswesen. Zur Logik der Leistungssteigerung und Effizienzmaximierung und ihren ethischen Grenzen« plädiert Arne Manzeschke schließlich dafür, Ressourcenallokationsprobleme nicht allein aus der Perspektive der Gesundheitsökonomie zu beantworten, sondern auch aus ethischer Sicht. 
Seiner Ansicht nach ist es Aufgabe der Ethik, Pfadabhängigkeiten und Automatismen disziplinären Denkens aufzudecken und den Blick für multiperspektivische Lösungswege zu weiten. Genau diese Zielsetzung verfolgen wir mit diesem Buch.

\section{LITERATUR}

Daniels, N. (1985): Just Health Care. Cambridge: Cambridge University Press.

Deutscher Ethikrat (2012): Nutzen und Kosten im Gesundheitswesen - Zur normativen Funktion ihrer Bewertung. Stellungnahme Januar 2011. In: Honnefelder, L.; Sturma, D. (in Verbindung mit Beckmann, J.P.; Horn, C.; Siep, L.) (Hrsg): Jahrbuch für Wissenschaft und Ethik 16. Berlin/Boston: de Gruyter, 379-439.

Drummond, M.F.; Sculpher, M.J.; Torrance, G.W.; O’Brien, B.J.; Stoddart, G.L. (2005): Methods for the Economic Evaluation of Health Care Programmes. 3. Auflage. Oxford: Oxford University Press.

Engelhardt, D.v. (1998): Artikel Gesundheit. In: Korff, W.; Beck, L.; Miktat, P. (Hg.): Lexikon der Bioethik, Bd. 2. Gütersloh: Gütersloher Verlagshaus, 108-114.

Fangerau, H. (2009): Genetics and the Value of Life: Historical Dimensions. Medicine Studies 1: 105-112.

Fangerau, H.; Martin, M. (2011): Konzepte von Gesundheit und Krankheit: Die Historizität elementarer Lebenserscheinungen zwischen Qualität und Quantität. In: Viehöver, W.; Wehling, P. (Hg.): Entgrenzung der Medizin. Von der Heilkunst zur Verbesserung des Menschen? Bielefeld: transcript, 51-66.

Fischer, J. (2013): Anerkennung, Medikalisierung und Gerechtigkeit. In: Fangerau, H.; Kessler, S. (Hg.): Anerkennung und Selbstkonstitution. Geisteswissenschaftliche Schlüsselkategorien in medizinischen Deutungszusammenhängen von Armut und Krankheit. Freiburg: Karl Alber (2013). 
Fleßa, S. (2005): Gesundheitsökonomik - Eine Einführung in das wirtschaftliche Denken für Mediziner. Berlin: Springer.

Gollaher, D. (1994): From ritual to science: the medical transformation of circumcision in America. Journal of Social History 28: 5-36.

Gosepath, S. (2004): Gleiche Gerechtigkeit. Grundlagen eines liberalen Egalitarismus. Frankfurt a.M.: Suhrkamp Verlag.

Gosepath, S. (2007): Kann das Gut Gesundheit gerecht verteilt werden? In: Nationaler Ethikrat (Hg.): Gesundheit für alle - wie lange noch? Rationierung und Gerechtigkeit im Gesundheitswesen. Jahrestagung des Nationalen Ethikrates 2006. Berlin, 19-33.

Herder-Dorneich, P. (1994): Ökonomische Theorie des Gesundheitswesens: Problemgeschichte, Problembereiche, theoretische Grundlagen. Baden-Baden: Nomos-Verlag.

Kielhorn, A.; Schulenburg, J.M.v.d. (2000): The Health Economics Handbook. Tattenhall: Adis International.

Labisch, A.; Spree, R. (Hg.) (2001): Krankenhaus-Report 19. Jahrhundert. Krankenhausträger, Krankenhausfinanzierung, Krankenhauspatienten. Frankfurt a.M.: Campus.

Lemke, T.; Kollek, R. (2011): Hintergründe, Dynamiken und Folgen der prädiktiven Diagnosti. In: Viehöver, W.; Wehling, P. (Hg.): Entgrenzung der Medizin. Von der Heilkunst zur Verbesserung des Menschen? Bielefeld: transcript, 163-194.

Lenk, C. (2010): Gesellschaftsvertrag und Recht auf öffentliche Gesundheitsversorgung. In: Heubel, F.; Kettner, M.; Manzeschke, A. (Hg.): Die Privatisierung von Krankenhäusern. Ethische Perspektiven. Wiesbaden: VS Verlag für Sozialwissenschaften, 101-115.

Lenk, C. (2011): Enhancement vor dem Hintergrund verschiedener Konzepte von Gesundheit und Krankheit. In: Viehöver, W.; Wehling, P. (Hg.): Entgrenzung der Medizin. Von der Heilkunst zur Verbesserung des Menschen. Bielefeld: transcript, 67-87.

Marckmann, G.; Liening, P.; Wiesing, U. (Hg.) (2003): Gerechte Gesundheitsversorgung. Ethische Grundpositionen zur Mittelverteilung im Gesundheitswesen. Stuttgart: Schattauer Verlag. 
Marckmann, G.; Siebert, U.; (2011): Nutzenmaximierung in der Gesundheitsversorgung. Eine ethische Problemskizze. In: Gethmann-Siefert, A.; Thiele, F. (Hg.): Ökonomie und Medizinethik. 2. Auflage. München: Wilhelm Fink, 111-139.

Nord, E.; Richardson, J.; Street, A.; Kuhse, H.; Singer, P. (1995): Who cares about cost? Does economic analysis impose or reflect social values? Health Policy 34 (2): 79-94.

Paul, N.W. (2006): Gesundheit und Krankheit. In: Schulz, S.; Steigleder, K.; Fangerau, H.; Paul, N.W. (Hg.): Geschichte, Theorie und Ethik der Medizin. Eine Einführung. Frankfurt a.M.: Suhrkamp, 131-142.

Porzsolt, F.; Weiss, M.; Dietrich, E.S.; Fangerau, H. (2010): Die Bewertung von Gesundheitsleistungen durch Ökonomen und Ärzte - Eine Analyse von Konzepten, Methoden und Instrumenten. Recht und Politik im Gesundheitswesen 16 (4): 83-93.

Rawls, J. (1999): A Theory of Justice. Revidierte Fassung. Cambridge, Massachusetts: Harvard University Press.

Reiter-Theil, S.; Graf-Baumann, T.; Kutzer, K.; Müller-Busch, H.C.; Stutzki, R.; Traue, H.C.; Willweber-Strumpf, A.; Zimmermann, M.; Zenz, M. (2008): Ethik-Charta der Deutschen Gesellschaft zum Studium des Schmerzes (DGSS). Schmerz 22: 191-206.

Ritter, G. (1998): Soziale Frage und Sozialpolitik in Deutschland seit Beginn des 19. Jahrhunderts (Otto von Freising-Vorlesungen der Katholischen Universität Eichstätt-Ingolstadt). Eichstätt: Leske + Budrich Verlag.

Rosentreter, M. (2011): Zwischen Selbstbestimmung und Systemsteuerung. Zur Inanspruchnahme von Leistungen der Gesetzlichen Krankenversicherung durch die Patienten. In: Groß, D.; Rosentreter, M. (Hg.): Der Patient und sein Behandler. Die Perspektive der Medical Humanities. Berlin: Lit Verlag, 17-38.

Rothschuh, K.E. (1978): Konzepte der Medizin in Vergangenheit und Gegenwart. Stuttgart: Hippokrates.

Rychlik, R. (1999): Gesundheitsökonomie. Stuttgart: Ferdinand Enke Verlag. 
Schäfer, D.; Frewer, A.; Schockenhoff, E.; Wetzstein, V. (Hg.) (2008): Gesundheitskonzepte im Wandel. Geschichte, Ethik und Gesellschaft. Geschichte und Philosophie der Medizin. Band 6. Stuttgart: Steiner Verlag.

Schmidt-Wilke, J. (2004): Nutzenmessung im Gesundheitswesen: Analyse der Instrumente vor dem Hintergrund zielfunktionsabhängiger Informationsverwendung. Wiesbaden: Deutscher UniversitätsVerlag.

Schöffski, O.; Schulenburg, J.M.v.d. (Hg.) (2008): Gesundheitsökonomische Evaluationen. Berlin: Springer.

Schomerus, G.; Matschinger, H.; Angermeyer, M.C. (2006): Preferences of the public regarding cutbacks in expenditure for patient care: Are there indications of discrimination against those with mental disorders? Social Psychiatry and Psychiatric Epidemiology 41(5): 369-377.

Seelmann, K. (2008): Recht auf Gesundheit? Über den Wandel juristischer Perspektiven auf das Gesundheitswesen. In: Schäfer, D.; Frewer, A.; Schockenhoff, E.; Wetzstein, V. (Hg.): Gesundheitskonzepte im Wandel. Geschichte, Ethik und Gesellschaft. Geschichte und Philosophie der Medizin. Band 6. Stuttgart: Steiner Verlag.

Simillis, C.; Symeonides, P.; Shorthouse, A.J.; Tekkis, P.P. (2010): A meta-analysis comparing conservative treatment versus acute appendectomy for complicated appendicitis (abscess or phlegmon). Surgery 147(6): 818-829.

Sloan, F.A. (Hg.) (1995) Valuing Health Care - Costs, Benefits, and Effectiveness of Pharmaceuticals and Other Medical Technologies. Cambridge: Cambridge University Press.

Sozialgesetzbuch (SGB) Fünftes Buch (V) - Gesetzliche Krankenversicherung, \35b Kosten-Nutzen-Bewertung von Arzneimitteln, zu finden unter: www.gesetze-im-internet.de/sgb_5/-_35b. html, Zugriff: am 02.04.2013.

Task Force on Circumcision (2012): Circumcision policy statement. Pediatrics 130(3): 585-586. 
UN-Resolution zur Erklärung der Menschenrechte (Resolution 217 A (III) der Generalversammlung vom 10. Dezember 1948, zu finden unter: www.ohchr.org/en/udhr/pages/language.aspx?langid=ger, Zugriff: 08.03 .2013 .

WHO, Präambel zur Verfassung der Weltgesundheitsorganisation (Preamble to the Constitution of the World Health Organization as adopted by the International Health Conference, New York, 19 June - 22 July 1946), zu finden unter: www.who.int/governance/ eb/who_constitution_en.pdf, Zugriff: o8.03.2013. 


\section{Die Bewertung von Gesundheitsleistungen}





\title{
Zentrale gesundheitsökonomische Bewertungsverfahren
}

\author{
Ein einführender Überblick in Anlehnung an Schöffski \\ und von der Schulenburg
}

Kathrin Dengler

\section{EINFÜHRUNG ${ }^{1}$}

Wenn gesundheitsökonomische Bewertungsverfahren diskutiert werden, wird häufig der Einwand geäußert, dass menschliches Leben nicht monetär bewertet werden dürfe, weil dies unmoralisch sei. Dieser Einwand ist rein intuitiv berechtigt, und doch wäre es ebenso unmoralisch, den Kosten, die gewisse Gesundheitsleistungen verursachen, gar keine Aufmerksamkeit zu schenken und diesen Aspekt gänzlich aus dem Gesundheitssystem auszuklammern, denn die (monetären) Ressourcen einer jeden Volkswirtschaft sind begrenzt und es wäre höchstwahrscheinlich nicht im gesamtgesellschaftlichen Interesse, wenn das gesamte Bruttoinlandsprodukt

1 | Dieser Überblick orientiert sich stark an den spezifischen Ausführungen in dem Sammelband Gesundheitsökonomische Evaluationen, der von Oliver Schöffski und J.-Matthias Graf von der Schulenburg im Jahr 2008 in einer dritten, vollständig überarbeiteten Auflage herausgegeben wurde. Zentrale Beiträge aus dem Sammelband, die für die hier vorgelegte Zusammenstellung von Bewertungsverfahren herangezogen wurden und auf die sich der folgende Überblick maßgeblich bezieht, sind: Greiner 2008; Greiner und Schöffski 2008; Schöffski 2008a sowie Schöffski 2008b. 
(BIP) (also die gesamte Wertschöpfung eines Jahres) in das Gesundheitssystem fließen würde. ${ }^{2}$ Ziel ist es daher, die zur Verfügung stehenden Mittel sinnvoll zu allozieren.

Hierzu werden auf verschieden Ebenen Entscheidungen getroffen ${ }^{3}$ : Auf politischer Ebene wird eine Art Globalbudget für Gesundheitsleistungen beschlossen; hier finden gesundheitsökonomische Analysen keine Betrachtung. ${ }^{4}$ Wenn jedoch innerhalb des medizinischen Versorgungssystems die Mittel zugeteilt werden sollen, sind konkrete gesundheitsökonomische Regeln oder Vorgaben notwendig. Diese Regeln können sich im Zeitverlauf ändern und unterschiedliche Formen und Ausprägungen annehmen. Gesundheitsökonomische Bewertungsverfahren sollen unter anderem dazu dienen, Transparenz im Prozess der Verteilung von zur Verfügung stehenden Ressourcen zu schaffen. Grundsätzlich lassen sich hier zwei Typen von Bewertungsverfahren unterscheiden: Solche, die nur den Mitteleinsatz betrachten und solche, die zusätzlich eine Ergebnisbetrachtung vollziehen.

Bevor in diesem Beitrag einzelne Bewertungsverfahren vorgestellt werden, soll eine Bestimmung der Kosten- und Nutzenbegriffe im Gesundheitswesen erfolgen. Im Anschluss an die Betrachtung der Evaluationsverfahren werden verschiedene Methoden zur

2 | Nach OECD-Gesundheitsdaten (OECD 2012) belief sich der Anteil der Gesundheitsausgaben am BIP im Jahr 2010 auf 11,6 \% und liegt dabei 2,1 Prozentpunkte über dem Schnitt der OECD-Länder.

3 | Vergleiche hierzu Teil 1 des Beitrags von Walter Pfannkuche in diesem Band.

4 | Wobei es natürlich durchaus spannend wäre, nicht nur zwischen verschiedenen Gesundheitsleistungen eine Art Kosten-/Nutzen-Analyse vorzunehmen, sondern eventuell auch zwischen verschiedenen Bereichen. So wäre es zumindest theoretisch möglich, die optimale Aufteilung der Mittel in die verschiedenen Ressorts (Bildung, Gesundheit, Infrastruktur...) zu finden oder Bereiche aufzuspüren, in denen die monetären Mittel mehr bewirken könnten als in anderen. 
Messung des Nutzwerts näher betrachtet und abschließend kommentiert.

\section{Definitorische Vorklärungen}

Die Bewertungssysteme zur Evaluation von Gesundheitsleistungen orientieren sich an den Kosten und teilweise am Nutzen der erbrachten Leistungen, wobei sowohl die Bestimmung der einzubeziehenden Kosten als auch gegebenenfalls die Bestimmung dessen, was konkret unter »Nutzen« zu verstehen ist, im Prinzip bei Berechnungen immer eindeutig und präzise angegeben werden sollte, da es unzählige Auslegungsvarianten für diese beiden Parameter gibt. Ohne diese präzisen Angaben bleiben gewichtige Prämissen intransparent und die Bewertungsergebnisse sind nicht nachvollziehbar.

Im Gesundheitswesen hängt die Zurechnung von Kosten und Nutzen auf bestimmte Leistungen in erster Linie von der Perspektive der jeweiligen Kosten- und/oder Nutzenanalyse ab. So sind im Wesentlichen drei Perspektiven zu unterscheiden: Erstens die volkswirtschaftliche Sicht, deren Ziel eine umfassende Betrachtung ist; $z$ weitens die Perspektive des Leistungserbringers, beispielsweise des Krankenhausmanagements oder des Arztes sowie drittens der Blickwinkel der Patienten. Die Unterscheidung dieser Perspektiven ist unter anderem deshalb relevant, weil die Einnahme dieser sich unterscheidenden Bewertungspositionen es mit sich bringt, dass der gesamte Ressourcenverzehr nicht immer allen relevanten Kosten entspricht, da beispielsweise aus der Perspektive des Krankenhauses nicht die entstehenden Kosten etwa durch den Arbeitsausfall des Patienten oder die nachfolgende Pflege zu Hause erfasst werden (vgl. Greiner 2008, S. 51-52).

Da auch die im Folgenden betrachteten gesundheitsökonomischen Evaluationsverfahren spezifische Perspektiven der Bewertung abbilden, werden hier kurz die Messgrößen »Kosten« und 
»Nutzen $\aleph^{5}$ vorgestellt, um eine einheitliche begriffliche Basis für die nachfolgenden Bewertungsverfahren zu schaffen. ${ }^{6}$

\subsection{Der Kostenbegriff im Gesundheitswesen ${ }^{7}$}

Wenn es darum geht, die Kosten medizinischer Therapieverfahren zu erfassen, muss zuallererst die Frage gestellt werden, welche Kosten erfasst werden sollen. Grundsätzlich lassen sich direkte und indirekte Kosten unterscheiden. Bei direkten Kosten handelt es sich um Kosten, die einem Bezugsobjekt unmittelbar zugerechnet werden können (z.B. Medikamente oder die Arbeitsstunden des Arztes). Indirekte Kosten können einem Bezugsobjekt hingegen nur mittelbar, bspw. über einen Schlüssel, zugerechnet werden (klassische Beispiele sind hier die Kosten für die Verwaltung eines Krankenhauses oder die Reinigung der Eingangshalle). Eine weitere Differenzierungsdimension stellt die Quantifizierbarkeit dar. Es gibt Kosten, beispielsweise das Gehalt des Arztes oder die Kosten für Medikamente, die klar monetär fassbar sind. Demgegenüber stehen jedoch Kosten, die nicht so klar in monetären Einheiten auszudrücken sind. Hierunter fallen beispielsweise Schmerzen, die während der Behandlung auftreten, Unwohlsein oder folgende Einschränkungen der Mobilität. Aber auch generelle Einbußen in der Lebensqualität oder Verringerungen der Produktivität zählen zu dieser Art von Kosten (vgl. hierzu Greiner 2008, S. 55). Bevor also eine gesundheitsökonomische Analyse durchgeführt werden kann, muss klar

5 Als Synonym zum Begriff des Nutzens findet sich häufig auch der Ausdruck "Outcome".

6 | Für weiterführende Informationen und Überlegungen zu Kosten und Nutzen in gesundheitsökonomischen Evaluation vgl. Greiner 2008.

7 | Eine detaillierte Ausführung zu Kosten und Nutzen im Gesundheitswesen findet sich beispielsweise bei Dranove 1995; Oberender 1995; Gold et al. 1996; Greiner 2008. 
definiert werden, welche Kosten als so genannte Input-Faktoren ${ }^{8}$ in die Analyse miteinbezogen werden. Der einfachste Weg besteht zumeist darin, nur klar quantifizierbare Kosten zu berücksichtigen.

\subsection{Der Nutzenbegriff im Gesundheitswesen}

Der Nutzenbegriff im Gesundheitswesen ist ebenfalls schwer fassbar, was das Problem mit sich bringt, dass eine Vielzahl an ähnlichen Begriffen zum Nutzen in der Literatur zu finden ist. Nachfolgend werden ein paar ausgewählte, häufig anzutreffende Begriffe, die alle eine Form von Nutzen charakterisieren, vorgestellt:

Unter dem Nutzen bzw. dem Benefit kann entweder ein Prinzip verstanden werden (»This principle asserts that we ought always to produce the maximal balance of good consequences over bad consequences « [Beauchamp et al. 2008, S. 12]) oder ein Ausdruck einer Präferenzstruktur. Hiernach ist Nutzen »a measure of the preference for, or desirability of, a specific level of health status or specific health outcome (Kielhorn et al. 2000, S. 164). Davon zu unterscheiden ist der Begriff des Nutzwerts (Utility). Dabei handelt es sich um eine Zielgröße, die versucht, den Behandlungserfolg einer Maßnahme aus Patientensicht zu bewerten, das heißt, es werden Effekte auf die Lebensqualität und die Lebenserwartung des Patienten berücksichtigt (vgl. Schöffski 2008a, S. 92). Der Begriff Beneficence (auch Wohlergehen/Wohltun) steht prinzipiell dafür, anderen Gutes zu tun. In vielen Moraltheorien steht er auch für die als moralische Verpflichtung, dem Mitmenschen Gutes zu tun (vgl. Savulescu 2007; Kahn und Mastroianni, 2007; Gillon 1998). Wenn es darum geht, die Wirksamkeit medizinischer Interventionen im Alltagsgeschehen zu überprüfen, wird meist von Effectiveness gesprochen. Sie beschreibt die »in der Realität der medizinischen Versorgung tat-

8 | Unter Input-Faktoren werden alle Mittel verstanden, die zur Bereitstellung oder Erstellung eines Gutes benötigt werden. An dieser Stelle erfolgt die verkürzte Betrachtung, in der nur die Kosten als relevant erachtet werden. 
sächlich erzielte bzw. erzielbare Effektivität« (Greiner 2008, S. 50). In Abgrenzung dazu gibt die Efficacy die Wirksamkeit einer medizinischen Maßnahme unter Optimalbedingungen an, also die »unter optimalen Bedingungen zu erreichende Effektivität« (Busse 2006, S. 7; vgl. auch Busse et al. 2002; Strech 2005; Schöffski 2008a). Es geht hier um die »klinische Wirksamkeit unter kontrollierten Bedingungen, also bei bestmöglicher Indikation und Anwendung « (Greiner 2008, S. 50; vgl. auch Busse et al. 2002; Strech 2005). Ein weiterer, häufig in diesem Feld anzutreffender Begriff ist der der Efficiency (Effizienz). Mit Efficiency ist »die unter Berücksichtigung der gegebenen Mittel optimale Produktion einer Gesundheitsleistung gemeint (Effizienz)« (Greiner 2008, S. 50).

Bei den im nächsten Abschnitt betrachteten zentralen ökonomischen Bewertungsverfahren finden sich die Begriffe Wirksamkeit (bei der Kosten-Wirksamkeits-Analyse), Nutzen (Kosten-NutzenAnalyse) und Nutzwert (Kosten-Nutzwert-Analyse) wieder.

\section{3. ÜBERBLICK: GRUNDFORMEN GESUNDHEITSÖKONOMISCHER EvalUationen}

Die Grundformen einer gesundheitsökonomischen Evaluation von Gesundheitsleistungen unterscheiden sich insbesondere darin, welche Kosten- und Nutzenkomponenten berücksichtigt werden und welche Vergleiche zwischen den eingesetzten Ressourcen und dem Ergebnis der Maßnahme gezogen werden. Dabei ist anzumerken, dass mithilfe der meisten Bewertungsmethoden sowohl Arzneimittel, aber auch alle anderen medizinischen Leistungen im Hinblick auf Kosten und Nutzen bewertet werden können (siehe bspw. Schöffski 2008a, S. 65ff.). Gleichzeitig können gesundheitsökonomische Bewertungsverfahren unterschiedlich systematisiert werden. Zum einen besteht die Möglichkeit, zwischen Studien ohne vergleichendem Charakter und Studien mit vergleichendem Charakter zu unterscheiden (so geht z.B. Schöffski 2008a vor). Zum anderen kann eine Unterscheidung anhand der Input-/Output-Per- 
spektive vorgenommen werden. Letzterer Systematisierung wird in diesem Beitrag gefolgt. Es werden Verfahren betrachtet, die nur die Seite des Mitteleinsatzes in den Blick nehmen (Input), und Verfahren beschrieben, die neben dem Mitteleinsatz auch eine Ergebnisperspektive (Output) berücksichtigen.

\subsection{Ausschließliche Betrachtung des Mitteleinsatzes (Input-Perspektive)}

Grundsätzlich kann bei einer ausschließlichen Betrachtung des Mitteleinsatzes bei einer Maßnahme erst einmal festgestellt werden, wie viel sie kostet. Dieser Frage gehen die Kosten-Analyse und die Krankheitskosten-Analyse nach. Für eine Optimierung der Ressourcenallokation im Gesundheitswesen sind generell Studien erforderlich, die eine gewisse Vergleichbarkeit ermöglichen. Wenn der Fokus jedoch nur auf dem Aspekt des Mitteleinsatzes liegt, also lediglich eine Input-Perspektive eingenommen wird, ist eine Vergleichbarkeit verschiedener Maßnahmen nur unter sehr eingeschränkten Bedingungen möglich. Der Output der verschiedenen Maßnahmen muss daher quasi gleich sein, da er nicht in die Bewertung einfließt. Nichtsdestotrotz finden sich Fragestellungen, in denen auch diese Art der Evaluationen ihre Berechtigung haben. Sinnvoll ist diese Evaluationsmöglichkeit, wenn zum Beispiel die Beschaffung von Mullbinden oder Desinfektionsmittel evaluiert werden soll. Kurzum, sie lohnt sich bei all jenen Produkten oder Leistungen, bei denen hohe Vergleichbarkeit und ein Massencharakter vorliegen. In dieser Kategorie erfolgt ein Vergleich zweier Maßnahmen mithilfe der Kosten-Kosten-Analyse.

\section{Kosten-Analyse}

Die Kosten-Analyse (cost analysis, cost identification analysis) ist die einfachste Form einer ökonomischen Evaluation, die so auch in jedem anderen betriebswirtschaftlichen Umfeld angewandt werden kann. Sie umfasst ausschließlich die Ermittlung der Kosten einer 
bestimmten Maßnahme, das heißt, den Input an Ressourcen (vgl. Schöffski 2008a, S. 66f. und Drummond et al. 2005, S. 2ff.).

Wichtig ist hier, wie oben bereits erwähnt, dass klar entschieden werden muss, welche Kosten berücksichtigt werden. Als Ergebnis einer Kosten-Analyse erhält man einen Betrag z.B. in Euro, der widerspiegelt, wie viel eine Behandlungsmethode kostet. Allein aus der Kenntnis dieser Kosten lässt sich jedoch keine Entscheidung für oder gegen die Methode treffen, da dazu ein Vergleich mit Alternativen notwendig wäre (wie es z.B. bei der Kosten-Kosten-Analyse erfolgt). Die Kosten-Analyse ist sehr einfach durchzuführen, denn schließlich erfasst sie nur die Kosten einer bestimmten Maßnahme.

\section{Krankheitskosten-Analyse}

Bei der Krankheitskosten-Analyse (cost-of-illness analysis) handelt es sich um einen Spezialfall der Kostenanalyse. Sie wird in Deutschland relativ häufig durchgeführt (vgl. Schöffski et al. 2008, S. 67f). Mithilfe dieser Analysemethode kann festgestellt werden, wie groß die Bedeutung einer Krankheit für die gesamte Gesellschaft ist (vgl. hierzu Meldungen der Tagespresse, beispielsweise der Bericht »Krankheitskosten in Deutschland - So teuer kommen dicke Menschen das Gesundheitssystem « von Danuta Szarek auf Focus Online aus dem Jahr 2012). Das Ziel dieser Analyseform besteht darin, zu erkennen, wie hoch die gesellschaftliche Relevanz und die volkswirtschaftlichen Kosten einer Krankheit sind, um so festzustellen, wo Forschungsgelder sinnvoll zu investieren sind bzw. wo konkret Handlungsbedarf besteht (näheres hierzu findet sich beispielsweise bei Drummond 1992; Hodgson 1994; Rice 1966; Kielhorn und der Schulenburg 2000; Rychlik 1999 und Schöffski 2008a).

Zusammenfassend lässt sich festhalten, dass es sich bei der Krankheitskosten-Analyse um eine spezielle Ausprägung der Kostenanalyse handelt, die es sich zum Ziel setzt, alle Kosten, die bei einer Krankheit erstehen, als Ganzes zu erfassen. Folglich müssen alle direkten und indirekten Kosten in vollem Umfang berücksichtig werden. 


\section{Die Kosten-Kosten-Analyse}

Die Kosten-Kosten-Analyse ist häufig auch unter der Bezeichnung Kostenminimierungs-Analyse oder Kostenvergleichsrechnung zu finden (cost-cost analysis, cost-minimization analysis). Hier handelt es sich um vergleichende Studien, bei denen herausgefunden werden soll, welche Maßnahme die kostengünstigste ist. Zuerst werden die relevanten Kostenbestandteile festgesetzt, danach werden sie bei allen zu vergleichenden Maßnahmen aufsummiert und schließlich miteinander verglichen. Wie schnell klar wird, ist diese Analyse nur dann möglich, wenn die Outputs bei allen zu vergleichenden Maßnahmen gleich sind, denn hier liegt der Focus rein auf den InputFaktoren (d.h. Kosten). Wenn Ergebnisgleichheit vorliegt, ist diese Analysemethode leicht und schnell durchführbar. Die Schwierigkeit aber liegt in der Realität darin, dass hier sehr selten exakte Ergebnisgleichheit vorherrscht (vgl. hierzu Schöffski 2008a, S. 79ff.; Fleßa 2005 und Rychlik 1999).

\subsection{Betrachtung des Mitteleinsatzes und des Ergebnisses (Input/Output-Betrachtung) ${ }^{9}$}

Bei vergleichenden ökonomischen Evaluationsstudien im Gesundheitswesen werden allerdings kaum nur die Kosten betrachtet, sondern es wird meist auch das Ergebnis einer medizinischen Intervention mitberücksichtigt. Die einzelnen Studienformen unterscheiden sich dabei hauptsächlich darin, wie dieses >medizinische Ergebnis< gemessen wird. Während der Begriff Kosten verhältnismäßig eindeutig verwendet wird, geht die Begrifflichkeit des Gegenstücks des Nutzens stark auseinander. Nutzen wird teilweise allgemein und umfassend verstanden, kann aber auch nur monetäre Einheiten umfassen.

9 | Die folgenden kurzen Ausführungen sind stark angelehnt an die ausführlichen Erläuterungen von Oliver Schöffski in: Schöffski 2008a. 


\section{Die Kosten-Nutzen-Analyse}

Die Kosten-Nutzen-Analyse (cost-benefit analysis, benefit-cost analysis) stellt die klassische Form der ökonomischen Analyse dar. Sie wird daher vor allem auch in Bereichen außerhalb des Gesundheitswesens regelmäßig angewendet ${ }^{10}$ (vgl. Schöffski 2008a, S. 81). Bei der standardmäßigen Kosten-Nutzen-Analyse wird zum einen der gesamte Input, also alle anfallenden Kosten, und zum anderen der Output gemessen. Die Besonderheit liegt nun hierin, dass auch der Output in monetären Einheiten, also in Geld gemessen wird (vgl. Schöffski 2008a, S. 82). Die klassische ökonomische Vorgehensweise, sowohl Kosten als auch Nutzen in Geldeinheiten zu bewerten, wurde hier einfach auf den Bereich der Gesundheitsökonomie übertragen (vgl. ebd., S. 81). Bei einem Investitionsprojekt oder der Anlage in einem Aktienfonds mag dieses Vorgehen sinnvoll sein, da hier das Ergebnis, also der Output auch real in Geldeinheiten anfällt. Problematisch wird es jedoch, den Nutzen einer medizinischen Maßnahme in Geldeinheiten zu bewerten: Wie ist beispielsweise der Nutzen einer neuen Hüfte für eine 90-jährige monetär zu bewerten? Oder wie bewertet man den Nutzen einer Grippeschutzimpfung in Geldeinheiten? Die Schwierigkeit derartige Nutzenbestimmungen in Geldeinheiten zu fassen, erklärt die häufig vorgebrachte Kritik an der monetären Bewertung von Ergebnissen medizinischer Maßnahmen. Auch andere Besonderheiten des Gesundheitssystems finden bei dieser Analysemethode keinerlei Betrachtung. So lässt sie etwa auch Fragen außer Acht, welcher Therapieform oder welcher Person in welchen Fällen des Behandlungsbedarfs eine Priorität eingeräumt wird oder bei wem sich welche Therapie »lohnt«. Gerade an diesen Bruchlinien besteht erhebliches ethisches Konfliktpotential (siehe die folgenden Kapitel dieses Bandes).

10 | Anwendungen finden Kosten-Nutzen-Analysen beispielsweise in der Investitionsrechnung, wenn berechnet wird, ob sich die Investition in ein Projekt lohnt. D.h. wenn die zu erwarteten monetären Rückflüsse den monetären Wert der Anfangsinvestition übersteigen. 
Das Prinzip der Kosten-Nutzen-Analyse lässt sich folgendermaßen umschreiben: Wenn der bewertete Nutzen einer Behandlungsmethode in Geldeinheiten erfasst wurde, dann können von ihm entweder die Kosten abgezogen werden oder er kann durch die Kosten dividiert werden und als Ergebnis bekommt man einen Saldo. Nach diesem Saldo können in absteigender Reihenfolge alle zu bewertenden Maßnahmen einem Ranking unterzogen werden und so ist eindeutig erkennbar, welche Maßnahmen den höchsten Saldo haben, also im Rahmen der angewandten Berechnungen am lohnenswertesten sind. Diese Anordnung der Alternativen ist zweifelsfrei nur eindimensional (denn die einzig erfasste Dimension ist die der Geldeinheiten). Effekte wie erhöhtes Wohlbefinden oder verstärkte Leistungsfähigkeit finden in diesem Maß keinerlei Berücksichtigung (vergleiche hierzu Schöffski 2008a, S. 81ff. und Drummond et al. 2005 , S. 2 ff.).

\section{Die Kosten-Wirksamkeits-Analyse}

Die Kosten-Wirksamkeits-Analyse (Kosten-Effektivitäts-Analyse, Cost-effectiveness analysis) berücksichtigt auch Effekte, die sich nicht problemlos in Geldeinheiten ausdrücken lassen, dabei werden nicht-monetäre Parameter in »naheliegenden natürlichen Einheiten « (Schöffski 2008a, S. 83; Hervorhebung im Original) gemessen. Naheliegende natürliche Einheiten sind etwa Parameter, die der Arzt am Körper des Patienten messen kann, d.h. Blutdruck, Leberwerte, Gewicht usw. Der Mediziner kann hierbei die Größe festlegen, anhand derer der Erfolg einer zu evaluierenden Maßnahme festgestellt werden soll (vgl. Schöffski 2008a, S. 83). Dieser Parameter liefert bei dieser Analysemethode die Output-Perspektive. Die InputPerspektive bilden wiederum die Kosten, die für die Behandlung anfallen. Im Endeffekt wird danach gefragt, wie viel Kosten pro Einheit des festgelegten Parameters entstehen. Ermöglicht wird im Rahmen dieses Analyseverfahrens die Beurteilung zweier Maßnahmen, die auf demselben Gebiet wirken sollen, jedoch unterschiedliche Kosten und unterschiedliche Ergebnisse haben. Wichtig ist, dass die Ergebnisdimension (also der Parameter, der vom Arzt fest- 
gelegt wird) jeweils derselbe ist (vgl. hierzu bspw. Schöffski 2008a; Rychlik 1999, S. 49; Drummond et al., S. 2 ff.).

Als Kritikpunkt an dieser Analysemethode kann angeführt werden, dass neben der Kostenperspektive nun die Perspektive des Arztes berücksichtigt wird, jedoch das Befinden des Patienten (das durchaus von dem Wert eines festgelegten Parameters abweichen kann) außen vor bleibt (vgl. Schöffski 2008a, S. 91). So schreibt beispielsweise Schöffski:

"Für den Patienten ist es erst einmal unerheblich, wie hoch der Blutdruck oder wie groß der Tumor ist. Für inn ist einzig und allein relevant, wie sich seine Lebensqualität und seine Lebenserwartung entwickelt" (Schöffski 2008a, S. 91).

Doch genau hier liegt das Problem: Wenn eine Behandlungsmethode für den Patienten wesentlich angenehmer ist oder mit weniger Schmerzen verbunden ist, dann ist das in der Kosten-WirksamkeitsAnalyse in der Regel vollkommen irrelevant. Was zählt, sind Kosten und Ergebnis (in Form eines technischen Parameters) (vgl. Schöffski 2008a, S. 91). Hinzu kommt, dass mithilfe dieser Methode keinerlei Vergleiche innerhalb des Gesundheitssystems möglich sind, da die Output-Perspektive von Fall zu Fall variiert. Für indikationsübergreifende, effiziente Allokationen (die schließlich gewünscht sind, sonst bestände keine Notwendigkeit für gesundheitsökonomische Evaluationen) ist diese Analyseform daher nur von eingeschränktem Nutzen (vgl. Schöffski 2008a, S. 91).

\section{Die Kosten-Nutzwert-Analyse}

An diesem Punkt unternimmt die Kosten-Nutzwert-Analyse (costutility analysis) den Versuch, der oben genannten Kritik an den anderen Bewertungsmethoden nachzukommen und eine neue Perspektive einzunehmen (vgl. Schöffski 2008a, S. 92). Schöffski bringt diese Zielsetzung der Kosten-Nutzwert-Analyse mit der Einschätzung auf den Punkt, dass hier »die Bewertung des Behandlungserfolgs einer medizinischen Maßnahme aus Patientensicht« 
erfolge, »d.h. es werden die Effekte auf die Lebensqualität und die Lebenserwartung des Patienten berücksichtigt« (Schöffski 2008a, S. 92). Dieser so ermittelte Nutzwert wird mit den anfallenden Kosten ins Verhältnis gesetzt (siehe hierzu u.a. Schöffski 2008a; Drummond 2005; Rychlik 1999; Kielhorn und Schulenburg 2000; Butler 1990 und Gerard 1992).

Wichtig bei dieser Analysemethode ist die Output-Dimension, der sogenannte Nutzwert. Es gibt verschiedene Verfahren, wie dieser ermittelt wird, häufig angewandt wird das Konzept der qualitätsadjustierten Lebensjahre (QALY). Dieses Konzept sowie mögliche Alternativen werden im folgenden Abschnitt kurz vorgestellt. Der große Vorteil an dieser Analyse (insbesondere gegenüber der Kosten-Wirksamkeits-Analyse) liegt darin, dass nun alle medizinischen Maßnahmen nach demselben Muster bewertet werden. Die Normierung in der Output-Perspektive (im Fall der qualitätsadjustierten Lebensjahre zum Beispiel Lebensqualität verknüpft mit Lebensdauer) ermöglicht eine indikationsübergreifende vergleichende Analyse aller möglichen Behandlungsmethoden. Alle Maßnahmen lassen sich mithilfe dreier Dimensionen, nämlich zum Beispiel Kosten, Lebensqualität und Lebensdauer bewerten.

\subsection{Verfahren zur Nutzwertmessung: Das QALY-Konzept und alternative Konzepte ${ }^{11}$}

\section{Messung des Nutzwertes}

Die Kosten-Nutzwert-Analyse stellt sich als ein Konzept dar, das sich vor allem darum bemüht, der Patientensicht gerecht zu werden. Hierfür müssen in der Dimension des Outputs, also des Ergebnisses der medizinischen Maßnahme, Nutzwerte ermittelt werden. Neben dem schon erwähnten Konzept der QALYs gibt es eine Reihe weiterer Alternativen, wie zum Beispiel die Healthy year equivalents

11 | Die folgenden Ausführungen zur Nutzwertmessung orientieren sich stark an Schöffski und Greiner 2008. 
(HYE), das Saved young life equivalent (SAVE)-Konzept und die Disability-adjusted life years (DALY).

\section{Das Konzept der qualitätskorrigierten Lebensjahre (QALYs)}

Das QALY-Konzept (quality-adjusted life-years) ist wohl die bekannteste Möglichkeit, Nutzwerte zu ermitteln. Das Ziel dieses Ansatzes ist es, eine Komplexitätsreduktion von einer Fülle an Informationen vorzunehmen (vgl. Schöffski und Greiner 2008, S. 95f.). Der Nutzwert besteht hierbei aus zwei Komponenten, der Lebensqualität und der restlichen Lebenserwartung. Die restliche Lebenserwartung ist eine relativ einfach $\mathrm{zu}$ bestimmende, quantitative Dimension. Es wird lediglich bestimmt, wie viele zusätzliche Lebensjahre der $\mathrm{zu}$ Behandelnde durch die medizinische Maßnahme zu erwarten hat. Die andere Komponente, die Lebensqualität, stellt den schwierigeren Aspekt dieses Konzeptes dar, denn in diesem Parameter sind selbst schon unterschiedliche Dimensionen mitinbegriffen (vgl. den Beitrag von Reinhold Kilian in diesem Band). Hier ist eine qualitative Bewertung nötig. Wer nach einer einfachen Bewertung seiner Lebensqualität gefragt wird, wird wohl große Schwierigkeiten haben, eine solche Frage zu beantworten. Aus diesem Grund werden hierzu in der Praxis mehr oder weniger standardisierte Methoden verwendet, um anhand verschiedener Teilaspekte möglicher Dimensionen und Konzeptionen von Lebensqualität ein sinnvolles Aggregat zu bekommen ${ }^{12}$. Von Vorteil ist hier, dass auch Aspekte wie beispielsweise eine unangenehme oder schmerzhafte Behandlung mit in die Bewertung einfließen können, da sie ja auch in einer Veränderung der Lebensqualität (in diesem Falle negativ) berücksichtigt werden können.

12 | Exemplarisch nennt Schöffski folgende mögliche Methoden: a) Telefoninterview, b) Fremdeinschätzung durch Freunde, Verwandte, c) Fremdeinschätzung durch einen Arzt, d) Persönliches Interview, e) selbstauszufüllender Fragebogen, f) Tagebuch und g) Internetbefragung (vgl. Schöffski 2008b, S. 326). 
Wenn nun die beiden Dimensionen Restlebensdauer und Lebensqualität erfasst sind, dann können sie zu einem neuen »eindimensionalen Outcome-Maß« (Schöffski und Greiner 2008, S. 97) zusammengefasst werden. Dieses sind die so genannten QALYs. Auf diese Weise werden indikationsübergreifende Vergleichsstudien ermöglicht, die die Patientenperspektive berücksichtigen (vgl. Schöffski und Greiner 2008, S. 97). Ein QALY kann somit zu einem für das gesamte Gesundheitswesen normierten Nutzenmaß werden. Wenn dieses Maß im Rahmen der Kosten-Nutzwert-Analyse verwendet wird, ist nach ökonomischer Logik der Maßnahme der Vorzug zu geben, die den größtmöglichen Nutzen pro Geldeinheit erbringt, also den höchsten Wert von QALYs pro Geldeinheit hat (vgl. Schöffski und Greiner 2008, S. 97).

\section{Healthy years equivalents (HYEs)}

Die healthy year equivalents stellen eine Alternative zum QALY-Konzept dar. Sie messen das Outcome von Gesundheitsmaßnahmen, indem sie zwei Dimensionen betrachten: Lebensqualität und Lebensdauer. Im Gegensatz zu dem QALYs (die auch mit diesen zwei Dimensionen operieren) repräsentieren HYEs jedoch die Präferenzen des Patienten (oder die anderer Individuen). Dies ist der Fall, da die Präferenzen direkt aus der individuellen Nutzenfunktion der Individuen berechnet werden (vgl. Mehrez und Gafni, 1991, S. 140). Die Grundidee ist hier, dass nach der Anzahl an Jahren mit perfekter Gesundheit (gefolgt vom Tod) gesucht wird, die denselben Nutzen stiftet wie ein lebenslanges Gesundheitsprofil, das einer zu bewertenden medizinischen Intervention folgt. Da das HYE-Konzept dabei direkt auf der individuellen Nutzenfunktion aufbaut, bedeutet das, dass diese zuerst festgestellt werden muss. Das zieht aufwendige Befragungen und lange Interviews mit sich. Die Machbarkeit einer solchen Studie hängt dabei von den finanziellen Ressourcen und der Bereitschaft der Individuen zur Teilnahme ab. Die Ermittlung der HYEs ist dementsprechend relativ komplex. Individuen müssen in einem mehrstufigen Verfahren verschiedene Gesundheitszustände hypothetisch bewerten; das ist zweifelsfrei mit 
einigen Schwierigkeiten belastet (vgl. hierzu Schöffski und Greiner 2008, S. 128ff.; Kielhorn und Schulenburg 2000, S. 81f.). Zur Anwendung kommt hier das sogenannte Standard-Gamble Verfahren ${ }^{13}$, das herangezogen wird, wenn individuelle Präferenzen eines Individuums unter Unsicherheit gemessen werden, um die Outcomes verschiedener therapeutischer Interventionen in Nutzwerten anzugeben. Diese Nutzwerte können dann bei der medizinischen Entscheidungsfindung berücksichtigt werden, um unterschiedliche Maßnahmen zu evaluieren (Gafni 1994, S. 207).

\section{Saved young life equivalent (SAVE-)Konzept}

Das SAVE-Konzept stellt eine alternative Evaluationsform von Gesundheitsleistungen dar, bei der es um die Wertschätzung der Gesellschaft eines speziellen Outcomes geht: dem geretteten jungen Leben (saving a young life). Die Einheit, mit der bei dieser Methode argumentiert wird, ist demnach auch als SAVE bezeichnet. Der Wert eines geretteten jungen Lebens dient als Referenzwert. Die Outcomes anderer Gesundheitsleistungen können dann einfach in die Einheit SAVE umgerechnet werden. Ebenso wie die QALYs kann bei den SAVEs ein Vergleich verschiedener Behandlungen durch Bildung einer Kosten-Nutzen-Relation angestellt werden. Jedoch ermöglicht das SAVE-Konzept im Gegensatz zu den QALYs die Berücksichtigung verschiedener ethischer Aspekte, z.B. verteilungspolitische Interessen (vgl. Nord 1992, S. 875). Wie Schöffski und Greiner explizieren, kommt es beim SAVE-Konzept im Gegen-

13 | Beim Standard-Gamble-Verfahren handelt es sich um einen Prozess mit dessen Hilfe Nutzwerte für Gesundheitszustände abgeleitet werden können, die dann in Nutzwert-Verfahren Verwendung finden. Um die Nutzwerte zu bestimmen, müssen Patienten zwischen einem bestimmten Krankheitszustand und einem "Spiel" entscheiden, bei dem es nur die beiden Alternativen a) komplette Genesung oder b) den Tod gibt. Die Eintrittswahrscheinlichkeiten der beiden Alternativen a) und b) werden so lange modifiziert, bis der Patient indifferent ist zwischen seinem Krankheitszustand und dem Spiel (Kielhorn und Schulenburg 2000, S. 166). 
satz zum QALY-Konzept nicht auf die individuelle Bewertung an, sondern es wird eine »soziale Evaluierung« vorgenommen (Schöffski und Greiner 2008, S. 133). Beim QALY-Konzept liegt der Fokus darauf, was man für sich selbst wählen würde, also auf dem eigenen Nutzen (vgl. Schöffski und Greiner 2008, S. 133). Das SAVE-Prinzip interessiert sich jedoch für die sozialen Präferenzen, was man also für andere Menschen wählen würde. Auch bei diesem Ansatz wird mit Probanden gearbeitet, denen zwei Alternativen vorgestellt werden, die zu gleichen Kosten führen (vgl. Schöffski und Greiner 2008, S. 133). Wenn Alternative 1 realisiert wird, wird in jedem Folgejahr das Leben eines jungen Menschen gerettet (wer genau als jung gilt, ist nicht näher spezifiziert). Alternative 2 steht für eine festgelegte Gesundheitsverbesserung in $n$ Fällen. Die Probanden müssen, wie Schöffski und Greiner hervorheben, nun jeweils entscheiden, wie hoch $n$ sein muss, damit die beiden Alternativen für sie gleichwertig sind. Je schlimmer die in Alternative 2 beschriebene Krankheit ist, desto kleiner ist tendenziell $n$ (vgl. Schöffski und Greiner 2008, S. 133).

Die beim SAVE-Konzept vorliegende Bindung an den Referenzwert eines geretteten jungen Lebens soll dazu dienen, ein besseres Verständnis bei den Adressaten der Kosten-Nutzwert-Analyse zu erreichen. Dieses Konzept stammt aus den skandinavischen Ländern (vgl. Schöffski und Greiner 2008, S. 133. Die Adressaten sollen im Idealfall nicht nur ihre eigenen Interessen, »sondern auch ethische und distributive Aspekte (Schöffski und Greiner 2008, S. 134) antizipieren. Gegen die Verwendung dieses Konzepts spricht, wie Schöffski und Greiner weiter ausführen, »dass die Probanden mit sehr detaillierten Informationen [...] versorgt werden müssen und sie diese häufig als medizinische Laien kaum einschätzen können« (Schöffski und Greiner 2008, S. 134). Außerdem ist das Verfahren noch nicht besonders etabliert; so weisen Schöffski und Greiner darauf hin, dass bisher »SAVEs bislang nur anhand verschiedener Schweregrade von Gehbehinderungen gemessen worden« sind (Schöffski und Greiner 2008, S. 134). 


\section{Disability-adjusted life years (DALY-)Konzept}

Zuletzt können DALYs als eine Möglichkeit der Bestimmung des Nutzwertes dienen. DALYs kombinieren dabei die verlorene Zeit durch den vorzeitigen Tod mit der Zeit, die man mit einer Erkrankung lebt (vgl. Schöffski und Greiner 2008, S. 135). Die verlorene Zeit durch den vorzeitigen Tod ergibt sich dadurch, dass ein Vergleich mit »eine[r] standardisierte[n] altersspezifischen Lebenserwartung « (Schöffski und Greiner 2008, S. 135) angestellt wird. Die Jahre, die mit der Krankheit gelebt werden, werden bei diesem Verfahren in Zeitverluste umgerechnet (vgl. Schöffski und Greiner 2008, S. 135). Damit soll, wie Schöffski und Greiner hervorheben, der eingeschränkten Funktionalität (oder Lebenstauglichkeit, Lebensqualität) durch die Erkrankung Rechnung getragen werden. Bei den DALYs wird somit ein »Schlecht« minimiert. Im Gegensatz dazu wird bei den QALYs ein »Gut«, wie Schöffski und Greiner schreiben, maximiert, denn es geht darum, möglichst viele qualitätsadjustierte Lebensjahre pro Kosteneinheit zu erzielen (vgl. Schöffski und Greiner 2008, S. 135).

Wie Schöffski und Greiner einleitend schreiben, wurde das Konzept der disability-adjusted-life-years (DALY) zu Beginn der 1990er Jahre im Umfeld der Weltbank und der Weltgesundheitsorganisation (WHO) entwickelt (vgl. Schöffski und Greiner 2008, S. 135). Das Konzept soll einen stark aggregierten Vergleich zwischen verschiedenen Ländern ermöglichen (vergleichbar einem Bruttoinlandsprodukt für Gesundheit) (vgl. ebd.).

\section{Abschliessende Betrachtung}

Generell lässt sich sowohl in der öffentlichen Debatte als auch in der wissenschaftlichen Literatur feststellen, dass gesundheitsökonomische Evaluationsmethoden international verstärkt zum Einsatz kommen. Jedoch ist zudem zu beobachten, dass die einzelnen Bewertungsmethoden ihren Einsatz in unterschiedlichen Ländern und in unterschiedlicher Ausprägung finden. So macht beispiels- 
weise Australien seit 1993 die Erstattungsfähigkeit von Medikamenten davon abhängig, wie kosteneffektiv diese sind. Umfassende Berücksichtigung finden gesundheitsökonomische Bewertungsverfahren unter anderem in Australien, den USA, Kanada und Großbritannien. Dort wird hauptsächlich die Kosten-Nutzwertanalyse angewandt, wobei die bevorzugte Ergebnisgröße die QALYs sind (vgl. Foos et al. 2010). Vor allem das Konzept der QALYs hat international eine große Debatte angestoßen. Häufiger Kritikpunkt ist, dass durch die Vereinheitlichung der Output-Perspektive auf den Parameter der qualitätskorrigierten Lebensjahre der Individualität des Einzelnen keinerlei Beachtung mehr geschenkt wird. Dieses eine Maß ist einfach für Berechnungen, doch schwierig zu generieren. Auf dem Weg der Berechnung, die der Komplexitätsreduktion dient, gehen viele relevante Informationen verloren. ${ }^{14}$

Darüber hinaus richten sich die verschiedenen Bewertungssysteme auf jeweils unterschiedliche Ergebnisdimensionen aus, d.h. sie setzen sich aus verschiedenen Messgrößen zusammen und verfolgen unterschiedliche übergeordnete Ziele. So wird entweder die Wirksamkeit, die Verträglichkeit, die Lebensqualität, die qualitätsadjustierten Lebensjahre, die Patientenzufriedenheit, die verbesserte Compliance oder der Grad der Arbeitsfähigkeit als Zielgröße genommen (vgl. Schulenburg et al. 2007).

Zusammenfassend lässt sich festhalten: Die Kostenanalyse verfolgt das Ziel der Kostenminimierung. Sie sagt allerdings nichts über die Wirksamkeit aus und auch nichts darüber, wie die Ressourcen zugeteilt werden sollen. Die Krankheitskostenanalyse hat die volkswirtschaftliche Ressourcenallokation zum Ziel. Allerdings stellt sich auch hier das Problem, dass die Betrachtung der Kosten nichts

14 | Vorstellen kann man sich hier beispielsweise einen professionellen Piano-Spieler, der eine Verletzung an der Hand hat. Eine komplette Wiederherstellung wird für inn wahrscheinlich einen wesentlich höheren subjektiven Wert haben, als für einen Sänger. Diese unterschiedliche Wertschätzung, basierend auf den individuellen Präferenzen, kann das Konzept der QALYs nicht berücksichtigen. 
darüber aussagt, ob überhaupt eine effektive Behandlungsmöglichkeit existiert. Die Kosten-Kosten-Analyse bevorzugt die kostengünstigere von zwei oder mehr vergleichbaren Gesundheitsleistungen. Sie ist aber nur sinnvoll bei Ergebnisgleichheit und gleichen Nebenwirkungen der untersuchten Gesundheitsleistungen, ansonsten ist sie ungeeignet. Die Kosten-Nutzen-Analyse stellt den Bewertenden vor die Herausforderung, den Nutzen in Geldeinheiten zu bewerten, was sehr umstritten ist - denn es erscheint schwierig bis unmöglich, zu bestimmen, wie viel z.B. ein niedrigerer Blutdruck wert ist. Die Kosten-Wirksamkeits-Analyse birgt die Schwierigkeit, dass auch nur Gesundheitsleistungen innerhalb einer Indikation verglichen werden können, da der Parameter Wirksamkeit keinen indikationsübergreifenden Vergleich zulässt.

Bei den genannten fünf Evaluationsinstrumenten erfolgt keine Berücksichtigung von Patientenpräferenzen. An diese Evaluationsvorgänge anschließende komplexere Konzepte versuchen diesen mehr Rechnung zu tragen: Das prominenteste Konzept ist hier die Berechnung von QALYs, die sowohl die Lebenserwartung als auch die Lebensqualität des Patienten berücksichtigen, dennoch treten auch hier Probleme auf, so zum Beispiel der Umstand, dass Ältere theoretisch aufgrund der geringeren Lebenserwartung weniger QALYs erreichen können als Jüngere (vgl. Schöffski und Greiner 2008, S. 12 of.). Außerdem werden lediglich Durchschnittspräferenzen berücksichtigt. Die HYEs messen ein Äquivalent in gesunden Lebensjahren, allerdings werden hier Durchschnittspräferenzen in einer künstlichen Entscheidungssituation gemessen, was als sehr problematisch angesehen wird. Das SAVE-Konzept misst den Referenzwert eines »jungen Lebens«. Hier findet eine soziale Evaluierung und keine individuelle Bewertung statt. Es wird demnach nach der gesamtwirtschaftlich erwünschten Ressourcenallokation gesucht. Allerdings ist es schwer festzulegen, bis wann ein Leben als »junges Leben« gilt. Außerdem hängt eine hypothetische Bewertung von Zuständen immer von der jeweiligen eigenen Position ab; diese kann sich jedoch im Zeitverlauf durchaus ändern, was einen nicht $\mathrm{zu}$ unterschätzenden Unsicherheitsfaktor darstellt. DALYS 
zuletzt stellen eine Kombination von verlorener Zeit und Leiden durch die Krankheit dar und messen somit die globale »Last« einer Krankheit. Nicht berücksichtigt werden hingegen die individuellen wirtschaftlichen und sozialen Umstände einer Person, die ebenfalls großen Einfluss auf die empfundene »Last« durch die Erkrankung haben. So ist es sicher >angenehmer<, in einem warmen Bett, umgeben von Familie und mit gutem Essen, krank zu sein und zu leiden, als auf einer kalten Liege, allein und hungernd.

Kurzum, die verschiedenen Verfahren haben unterschiedliche Herangehensweisen, um den Nutzwert einer Gesundheitsleistung festzustellen. Sie alle zielen darauf ab, komplexe Informationen zu verdichten, um so die Entscheidungssituation zu vereinfachen.

\section{LITERATUR}

Beauchamp, T.L.; Walters, L.; Kahn, J.P.; Mastroianni, A.C. (2008): Contemporary Issues in Bioethics. Belmont: Thomson Wadsworth.

Busse, R.; Orvain, J.; Velasco, M.; Perleth, M.; Drummond, M.; Gürtner, F.; Jørgensen,T.; Jovell, A.; Malone, J.; Rüther, A.; Wild, C. (2002): Best Practice in Undertaking and Reporting Health Technology Assesments. International Journal of Technology Assessment in Health Care 18 (2): 361-422.

Busse, R. (2006): Gesundheitsökonomie: Ziele, Methodik und Relevanz. Bundesgesundheitsblatt - Gesundheitsforschung - Gesundheitsschutz 49(1): 3-10.

Butler, J.R.G. (1990): Welfare Economics and Cost-Utility Analysis. In: Zweifel, P.; Frech, H.E. (Hg.): Health economics worldwide. Dordrecht: Kluwer, 143-157.

Dranove, D. (1995): Measuring costs. In: Sloan, F.A. (Hg.): Valuing Health Care-Costs, Benefits, and Effectiveness of Pharmaceuticals and Other Medical Technologies. Cambridge: Cambridge University Press.

Drummond, M.F. (1992): Cost-of-Illness Studies - A Major Headache. Pharmacoeconomics 2 (1): 1-4. 
Drummond, M.F.; Sculpher, M.J.; Torrance, G.W.; O’Brien, B.J.; Stoddart, G.L.(2005): Methods for the Economic Evaluation of Health Care Programmes. 3. Auflage. Oxford: Oxford University Press.

Fleßa, S. (2005): Gesundheitsökonomik - Eine Einführung in das wirtschaftliche Denken für Mediziner. Berlin: Springer

Foos, V.; Repschläger, U.; Riedel, R. (2010): Gutachten zu KostenNutzen-Bewertungsverfahren (KNB) für Arzneimittel in Deutschland und im internationalen Vergleich. Köln: Rheinische Fachhochschule Köln gGmbH.

Gafni, A. (1994): The Standard Gamble Method: What is Being Measured and how it is interpreted. Health Service Research 29(2): 207-224.

Gerard, K. (1992): Cost-utility in practice: a policy maker's guide to the state of the art. Health Policy 21: 249-279.

Gillon, R. (1998): Bioethics, Overview. In: Chadwick, R. (Hrsg): Encyclopedia of Applied Ethics, Volume 1. San Diego: Academic Press, 305.

Gold, M.R.; Siegel, J.E.; Russell, L.B.; Weinstein, M.C. (1996): CostEffectiveness in Health and Medicine. Oxford: Oxford University Press.

Greiner, W. (2008): Die Berechnung von Kosten und Nutzen. In: Schöffski, O.; Schulenburg, J.M.v.d.: Gesundheitsökonomische Evaluationen. Berlin: Springer, 49-62.

Hodgson, T. (1994): Cost of Illness in Cost-Effectiveness Analysis: A Review of the Methodology. Pharmacoeconomics 6 (6): 536-52.

Kahn, J.; Mastroianni, A. (2007): The Implications of Public Health for Bioethics. In: Steinbock, B. (Hg.): The Oxford Handbook of Bioethics. New York: Oxford University Press, 674.

Kielhorn, A.; Schulenburg, J.M.v.d. (2000): The Health Economics Handbook. Tattenhall: Adis International.

Mehrez, A.; Gafni, A. (1991): The Healths-years Equivalents: How to measure them Using the Standard Gamble Approach. Medical Decision Making 11: 140-146. 
Nord, E.(1992): An Alternative to QALYs: the saved young life equivalent (SAVE). British Medical Journal 305: 875-877.

Oberender, P. (Hg.) (1995): Kosten-Nutzen-Analysen in der Pharmaökonomie, Möglichkeiten und Grenzen. Gräfelfing: Socio-Medico.

OECD (2012): OECD-Gesundheitsdaten 2012. $\mathrm{Zu}$ finden unter: www.oecd.org/germany/BriefingNoteDEUTSCHLAND2012in German.pdf, Zugriff: 02.04.2013.

Rice, D.P. (1966): Estimating Cost of Illness. Washington, DC: United States Government Printing Office.

Rychlik, R. (1999): Gesundheitsökonomie. Stuttgart: Ferdinand Enke Verlag.

Savulescu, J. (2007): Genetic Interventions and the Ethics of Enhancement of Human Beings. In: Steinbock, B. (Hg.): The Oxford Handbook of Bioethics. New York: Oxford University Press, 523.

Schöffski, O.; Greiner, W. (2008): Das QALY-Konzept als prominentester Vertreter der Kosten-Nutzwert-Analyse. In: Schöffski, O.; Schulenburg, J.M.v.d. (Hg.): Gesundheitsökonomische Evaluationen. Berlin: Springer, 95-138.

Schöffski, O.; Schulenburg, J.M.v.d. (2008) (Hg.): Gesundheitsökonomische Evaluationen. Berlin: Springer.

Schöffski, O. (2008a): Grundformen gesundheitsökonomischer Evaluationen. In: Schöffski, O.; Schulenburg, J.M.v.d. (Hg.): Gesundheitsökonomische Evaluationen. Berlin: Springer, 65-92.

Schöffski, O. (2008b): Lebensqualität als Ergebnisparameter in gesundheitsökonomischen Studien. In: Schöffski, O.; Schulenburg, J.M.v.d. (Hg.): Gesundheitsökonomische Evaluationen. Berlin: Springer, 65-92.

Schulenburg, J.M.v.d.; Greiner, W.; Jost, F.; Klusen, N.; Kubin, M.; Leidl, R.; Mittendorf, T.; Rebscher, H.; Schöffski, O.; Vauth, C.; Volmer, T.; Wahler, S.; Wasem, J.; Weber, C., Mitglieder des Hannoveraner Konsens (2007): Deutsche Empfehlungen zur gesundheitsökonomischen Evaluation - dritte und aktualisierte Fassung des Hannoveraner Konsens. Gesundheitsökonomie \& Qualitätsmanagement 12: 285-290. 
Sloan, F.A. (Hg.) (1995): Valuing Health Care - Costs, Benefits, and Effectiveness of Pharmaceuticals and Other Medical Technologies. Cambridge: Cambridge University Press.

Strech, D. (2005): Der Umgang mit Wahrscheinlichkeiten und das Vertrauen in die Medizin - Ethische und wissenschaftstheoretische Aspekte einer Evidenz-basierten Medizin am Beispiel der Krebsfrüherkennung. Ethik in der Medizin 17: 103-113.

Szarek, D. (2012): Krankheitskosten in Deutschland - So teuer kommen dicke Menschen das Gesundheitssystem. Focus Online. Zu finden unter: www.focus.de/finanzen/versicherungen/krankenversi cherung/krankheitskosten-in-deutschland-so-teuer-kommendicke-menschen-das-gesundheitssystem_aid_844652.html, Zugriff: 15.11.2012.

Zweifel, P.; Frech, H.E. (Hg.) (1990): Health economics worldwide. Dordrecht: Kluwer 


\section{Beschreibungsdimensionen für gesundheitsökonomische Bewertungsverfahren}

Eine einführende Diskussion von Zielgrößen, Implikationen und ethischen Problemfeldern

Kathrin Dengler und Heiner Fangerau

\section{EINFÜHRUNG}

Gesundheitsleistungen können mittels unterschiedlicher (ökonomischer) Verfahren bewertet werden. ${ }^{1}$ Dabei stellen beispielsweise entweder die Kosten, das Verhältnis von Kosten und Nutzen, der Nutzwert oder auch die Wirksamkeit einer medizinischen Leistung die zu messende Zielgröße dar. Diese Zielgrößen variieren je nach dem gewähltem gesundheitsökonomischen Bewertungsansatz (vgl. Schöffski 2008). Oftmals basieren die Verfahren zur Evaluation von Gesundheitsleistungen auf dem Grundgedanken einer Maximierung von Gesundheit bei möglichst vielen Menschen (vgl. Marckmann und Siebert 2011). Die Größe und die Charakteristika der in Frage stehenden Gruppe sind dabei näherzu bestimmen. So ist es etwa denkbar, dass auf der einen Seite nur bei einer bestimmten Patientengruppe mit einem konkreten Krankheitsbild Gesundheitsverbesserungen erfasst werden sollen oder auf der anderen Seite der

1 Siehe hierzu die vorgestellten Bewertungsverfahren im Beitrag von Kathrin Dengler in diesem Band. 
Nutzen von Gesundheitsleistungen in Bezug auf eine ganze Gesellschaft (kategorial zum Beispiel als Staat, Bundesstaat oder Stadt gefasst) bestimmt werden soll. ${ }^{2}$

Im deutschen Gesundheitswesen stellt nach Oliver Schöffski die Etablierung und Nutzung von speziell gesundheitsökonomischen Evaluationen ein relativ junges Phänomen dar (vgl. Schöffski 2008, S. 8). ${ }^{3}$ Die Kosten des medizinischen Fortschritts, Medikalisierungsprozesse, der demographische Wandel (vgl. Marckmann 2003, S. 334), die staatliche Überschuldung und in der Folge immer wieder an ihre Grenze gelangende Sozialsysteme haben jedoch die Vorzeichen eines zunehmenden Ressourcenengpasses im Gesundheitswesen verstärkt (siehe auch Schmidt-Wilke 2004). Zumindest wird ein Bedarf an Rationalisierung, Priorisierung und Rationierung innerhalb des Gesundheitssystems konstatiert (vgl. Deutscher Ethikrat 2012, S. 384 ff.). Vor diesem Hintergrund scheint es folgerichtig, dass auch in der Bundesrepublik Deutschland nun vermehrt Ansätze zur Evaluation von Gesundheitsleistungen in die Debatte um Zuteilung mit einbezogen werden.

Schöffski benennt hierfür »zwei Gründe« (Schöffski 2008, S. 9):

"Zum einen kann es sein, dass mit den Ergebnissen [von Evaluationen] Entscheidungen über die Verwendung der evaluierten Maßnahmen beeinflusst werden können (in welcher Form auch immer), zum anderen ist es möglich, dass Vorschriften existieren, die die Durchführung [von Evaluationen] zwingend vorschreiben“ (Schöffski 2008, S. 9).

2 | Vgl. hierzu etwa die Prioritätenliste, die im US-amerikanischen Bundesstaat Oregon erstellt wurde mit dem Ziel, einen optimalen Ressourceneinsatz in der Gesundheitsversorgung zu etablieren. Vgl. Marckmann und Siebert 2002; Ubel et al. 1996; Nord 1993.

3 | Für einen kurzen historischen Überblick über die Entwicklung der Gesundheitsökonomie (in Deutschland) vgl. Schöffski 2008, S. $17 \mathrm{ff}$. 
In dieser Lesart werden gesundheitsökonomische Bewertungen von Gesundheitsleistungen genutzt, um den Weg für Entscheidungen über ihren Einsatz zu bahnen. Gesundheitsökonomische Evaluationen haben so ein großes Gewicht. In Ländern wie zum Beispiel Kanada, Australien, Schweden oder Großbritannien (vgl. Schöffski 2008, S. 7 fff.) werden gesundheitsökonomische Evaluationen im Kontext der Bestimmung der Erstattungsfähigkeit von medizinischen Mitteln vorgeschrieben. Im Sozialgesetzbuch (SGB) in Deutschland sind, wie Schöffski hervorhebt, ebenfalls Forderungen enthalten, die eine Wirtschaftlichkeitsprüfung verlangen (vgl. Schöffski 2008, S. 10). Auch das Institut für Qualität und Wirtschaftlichkeit im Gesundheitswesen (IQWiG) wendet für die Preisfestsetzung ein Effizienzgrenzenkonzept an (vgl. Deutscher Ethikrat 2012, S. $396 \mathrm{ff}$.).

Der zunehmende Gebrauch von gesundheitsökonomischen Verfahren und Methoden im Gesundheitsbereich - auch mit dem Ziel einer Rationierung von Leistungen - läßt es notwendig erscheinen, die Aussagekraft und Zielstellung dieser Bewertungsansätze zu prüfen (vgl. exemplarisch Deutscher Ethikrat 2012; Schmidt-Wilke 2004). Dabei ist sowohl danach $z u$ fragen, an welchen Zielgrößen sich die jeweiligen Verfahren orientieren und was jeweils gemessen wird, als auch zu prüfen, welche Faktoren und Bewertungsdimensionen keine oder nur wenig Berücksichtigung finden. Eine solche Betrachtung anzuregen, stellt das Ziel dieses Beitrags dar, wobei die hier vorgelegteDarstellung von ausgewählten Dimensionen und Zielgrößen keinen Anspruch auf Vollständigkeit erheben kann. Vielmehr soll die hier vorgelegte einführende Analyse exemplarisch einzelne auffällige Aspekte herausgegreifen und - u.a. in Anlehnung an eine Stellungnahme des Deutschen Ethikrates zur normativen Funktion der Bewertung von Nutzen und Kosten im Gesundheitswesen (Deutscher Ethikrat 2012) - einzelne auffällige Aspekte herausgreifen und erörtern. 


\section{SPANNUNGSFELDER IN GESUNDHEITSÖKONOMISCHEN BEWERTUNGSSYSTEMEN ${ }^{4}$}

Zunächst ist zu konstatieren, dass die verschiedenen Bewertungssysteme sich auf jeweils unterschiedliche Ergebnisdimensionen ausrichten, d.h sie setzen sich aus verschiedenen Meßgrößen zusammen und verfolgen unterschiedliche übergeordnete, nicht unbedingt miteinander vergleichbare Ziele. So werden entweder die Wirksamkeit, die Verträglichkeit, die Lebensqualität des Patienten, die qualitätsadjustierten Lebensjahre, die Patientenzufriedenheit, die verbesserte Compliance oder der Grad der Arbeitsfähigkeit als Zielgrößen erfasst (vgl. Schulenburg et al. 2007). Bei den fünf Evaluationsinstrumenten der 1) Kosten-Analyse, 2) KrankheitskostenAnalyse, 3) Kosten-Kosten-Analyse, 4) Kosten-Nutzen-Analyse und 5) Kosten-Wirksamkeits-Analyse (siehe der Artikel von Kathrin Dengler in diesem Band) erfolgt keine Berücksichtigung von Patientenpräferenzen innerhalb des Rechenmodells bzw. werden diese nicht abgefragt. Diese Lücke versuchen Kosten-Nutzwert-Analysen (wie sie zum Beispiel das QALY-Konzept, der Ansatz der HYE oder der DALY, sowie das SAVE-Konzept darstellen) zu schließen, indem sie den Wünschen, Bedürfnissen, Interessen und Präferenzen der Patienten bzw. Bürger mehr Rechnung tragen. Dabei werden diese Präferenzen jedoch in differenter Weise erhoben. Vielfach ist offen, wer genau befragt worden ist, wird oder werden soll (direkt von Krankheiten Betroffene, Angehörige, Menschen aus medizinischen Berufen usw.). Je nach gewählter Befragungsgruppe können die Aussagen mitunter stark variieren (vgl. hierfür die Ausührungen des Deutschen Ethikrates zur Bestimmung der Lebensqualität bei den QALYs in: Deutscher Ethikrat 2012, S. 395 sowie S. 407, dort vor allem auch Fußnote 72).

4| Eine ausgiebige Vorstellung der hier diskutierten Kosten-Nutzwert-Verfahren findet sich im vorangegangenen Beitrag von Kathrin Dengler. Um Redundanzen zu vermeiden, verweisen wir an dieser Stelle lediglich auf die dortigen Ausführungen und die dort verwendete Literatur. 
Insgesamt gilt, dass es von den Befragungsmethoden und -ansätzen abhängt, welche Bewertungsergebnisse am Ende zum Beispiel in Bezug auf eine Veränderung des Gesundheitszustandes durch eine bestimmte Therapie herauskommen. So schreibt der Deutsche Ethikrat in seiner oben erwähnten Stellungnahme in Bezug auf die Ermittlung derQALYs: »Insbesondere führen unterschiedliche Erhebungsverfahren für dasselbe Befragungsziel zu unterschiedlichen Ergebnissen, ohne dass man sagen könnte, welches Verfahren >besser misst « (Deutscher Ethikrat 2012, S. 407, Fußnote 72). Je nach getroffener Wahl entstehen also andere Evaluationsergebnisse, die es unserer Ansicht nach in pluralistischdemokratischen Gesellschaften zu explizieren, transparent zu machen und zu diskutieren gilt. Folglich ist nicht nur die Art und Weise der Präferenzerhebung innerhalb von gesundheitsökonomischen Bewertungsverfahren zu begründen und zu rechtfertigen, sondern gleichfalls auch schon die Wahl der gesundheitsökonomischen Evaluationsmethode selbst.

\section{ZiELGRÖSSEN UND MÖGLICHE KONFLIKTFELDER VON KOSTEN-NUTZWERT-VERFAHREN - EINE EXEMPLARISCHE BETRACHTUNG}

\subsection{Beschreibungsdimensionen von Nutzwert-Verfahren - Ein Vorschlag}

Die Kosten-Nutzwert-Verfahren fokussieren jeweils auf unterschiedliche Zielgrößen und berücksichtigen verschiedene Wertmaßstäbe. Im Folgenden sollen in exemplarischer Form acht Beschreibungsdimensionen vorgestellt werden, mit denen Kosten-Nutzwert-Verfahren charakterisiert und einer über ökonomische Evaluationen hinausgehenden Betrachtung zugänglich gemacht werden können. Die Darstellung ist offen und gelegentlich rhetorisch zugespitzt konzipiert, um Brisanz, Komplexität und dilemmatische Grundkonstellationen zu verdeutlichen. Sie erhebt dabei keinen Anspruch auf eine 
vollständige Wiedergabe ethischer Konflikt- und Problemfelder. Vielmehr soll sie als Diskussionsbasis und als Beitrag für eine Auseinandersetzung zur Frage nach einer gerechten Rationierung von Gesundheitsleistungen verstanden werden. Exemplarisch werden acht Beschreibungsdimensionen skizziert (siehe Abbildung 1) und einige mit ihnen assoziierte Problemfelder diskutiert. Die Beschreibungsdimensionen und Problemfelder wurden aus der Vielzahl der betroffenen Aspekte, die in der Literatur verschiedentlich verhandelt werden, ${ }^{5}$ von den Autoren in Zusammenarbeit mit Uta Bittner nach einem Workshop mit den anderen Beiträgern zu diesem Band und unter Bezugnahme auf die schon zitierte Stellungnahme des Deutschen Ethikrates (Deutscher Ethikrat 2012) als besonders relevant und hervorstechend ausgewählt. Kernpunkte und Grundfragestellungen der acht Beschreibungsdimensionen werden zudem in den folgenden Beiträgen dieses Bandes exemplarisch aufgegriffen, vertiefend erörtert und diskutiert. Andere Akzentuierungen bei der Auswahl von Beschreibungsdimensionen sind möglich. Außerdem sei an dieser Stelle darauf hingewiesen, dass die Dimensonen als auf unterschiedlichen Ebenen der Betrachtung liegend aufgefasst werden können. Dies wird von den Autoren weniger als methodischer Nachteil gewertet, sondern vielmehr als Perspektivenerweiterung und Auffächerung der Thematik angesehen.

5 | Siehe zum Beispiel die Bewertung von Nutzenmessungsverfahren bei Schmidt-Wilke 2004. Schmidt-Wilke arbeitet mit insgesamt sieben Bewertungskriterien zur Evaluation von Nutzenmessungsinstrumenten: „Im einzelnen sind dies Transparenz, Objektivität, Vollständigkeit, Genauigkeit, Vergleichbarkeit, geringer Aufwand und Ethik" (Schmidt-Wilke 2004, S. 197). Schmidt-Wilke bewertet die von ihr untersuchten Nutzenmessungsverfahren mit diesen sieben Kriterien u.a. in Bezug auf ihre Tauglichkeit bzw. Angemessenheit für eine "zielfunktionsabhängige Informationsverwendung", die sie in die drei Bereiche konzeptionelle, instrumentelle und symbolische Informationsverwendung einteilt (Schmidt-Wilke 2004). 


\subsection{Erläuterung der Beschreibungsdimensionen}

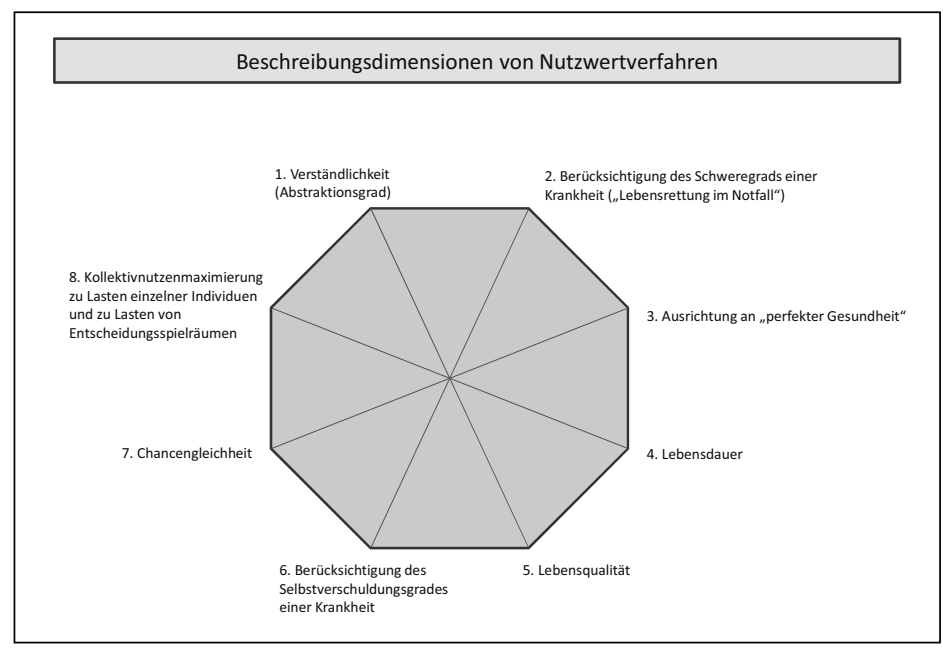

Abbildung 1: Acht Beschreibungsdimensionen von Nutzwertverfahren

\subsubsection{Verständlichkeit (Abstraktionsgrad)}

Mit dem Abstraktionsgrad ist die intuitive Verständlichkeit bei der Bewertung bzw. Evaluierung der Präferenzen und Priorisierungen gemeint. In Anlehnung an Schmidt-Wilke können hier auch die Kriterien der »Transparenz«, »Vollständigkeit« oder auch »geringer Aufwand« subsummiert bzw. mit einbezogen werden (SchmidtWilke 2004, S. 197-206). Die Dimension der Verständlichkeit orientiert sich daran, a) wie leicht die angestellten Berechnungen und Erhebungen der Präferenzen für den Laien nachvollziehbar sind, b) welches Reflexionsvermögen als notwendig vorausgesetzt wird und c) wie groß der Aufklärungsumfang und Erläuterungsaufwand zur Durchführung von Bewertungsmethoden ist. ${ }^{6}$

6 | Für eine ausführliche Analyse verschiedener Kosten-Nutzen-Messungsmethoden siehe die detaillierte Ausarbeitung bei der schon öfter zitierten Schmidt-Wilke 2004. Es sei angemerkt, dass Schmidt-Wilke andere Definitionen für Nutzenmessungsmethoden verwendet als die in diesem Bei- 
Als Beispiel für intuitive Verständlichkeit kann genannt werden, dass beim SAVE-Verfahren mitunter hervorgehoben wird, dass es von Vorteil sei, dass die Befragten sich eine Situation im Vergleich zu einem geretteten jungen Leben vorstellen sollen. So beschreibt Erik Nord zu den Hauptvorteilen von SAVE (vgl. Nord 1992, S. 877): »use easily understandable unit of measurement - namely, value of saving a young life« (Nord 1992, S. 877). Andere Berechnungsverfahren sind ebenfalls entsprechend ihrer Komplexität und Übersichtlichkeit zu evaluieren, sodass sich die relative und absolute Verständlichkeit jeweils bestimmen lässt.?

\subsubsection{Berücksichtigung des Schweregrades einer Krankheit ${ }^{8}$}

Im Gegensatz zum QALY-Verfahren wird beispielsweise bei der SAVE-Methode, wie Nord expliziert, auch der Schweregrad der Krankheit berücksichtigt (vgl. Nord 1992, S. 876). Wenn etwa die befragten Personen eine Verbesserung von einem sehr schlechten Zustand zu einem schlechten Zustand wichtiger finden als eine Verbesserung von einem guten zu einem sehr guten Zustand, dann kann dies, wie Nord ausführt, über das Äquivalent der SAVEs abgebildet werden (vgl. Nord 1992, S. 876).

Eine allgemeine, intuitive Einstellung der Bürger scheint $\mathrm{zu}$ sein, dass lebensrettende Maßnahmen stärker gewichtet werden als Maßnahmen, die einen ohnehin schon guten Gesundheitszustand verbessern (vgl. Deutscher Ethikrat 2012, S. 408-410). Auch Eva-

trag und im Beitrag von Kathrin Dengler in diesem Band gewählten. Vgl. Schmidt-Wilke 2004.

7 | Siehe den ähnlich gelagerten Ansatz von Schmidt-Wilke 2004.

8 | Der Deutsche Ethikrat benennt die "Missachtung der Relevanz des Schweregrads der Erkrankung“ (Deutscher Ethikrat 2012, S. 408) als einen zentralen Kritikpunkt am QALY-Konzept. Dieser Einwand sowie seine anschließende Diskussion innerhalb der Stellungnahme des Deutschen Ethikrates (vgl. Deutscher Ethikrat 2012, S. 408-410) bilden die Grundlage der folgenden Überlegungen zur zweiten Beschreibungsdimension. 
luationsverfahren sollten diesen Gedanken im Kern berücksichtigen. QALY-Gewinne etwa von 0,1 auf 0,2 müssten höher bewertet werden als QALY-Zuwächse von ०,8 auf ०,9 (vgl. Marckmann 2011, S. 135). In plakativer Form könnten hier die Lebensrettung am Unfallort auf der einen Seite (angenommen, dies entspricht hypothetisch einer QALY-Steigerung von 0,1 auf 0,2 ) und die ästhetische Nasenkorrektur wegen leichterer psychischer Belastung (angenommen, dies entspricht hypothetisch einer QALY-Steigerung von 0,9 auf 1) einander gegenübergestellt werden. ${ }^{9}$ Beide Gesundheitsleistungen leisten eine QALY-Verbesserung von o,1 Punkten, aber beide Leistungen sind in der Frage der Priorität der Leistung noch zusätzlich anders zu gewichten. Hieraus folgt die Notwendigkeit einer Ergänzung zum Beispiel des QALY-Modells, und zwar in dem Sinne, dass zunächst die Schlechtestgestellten zuvorderst behandelt werden sollten und somit prioritär Gesundheitsleistungen erhalten sollten (vgl. Deutscher Ethikrat 2012, S. 408f.). Erst dann sollen diejenigen Gesundheitsleistungen erhalten, denen es im Vergleich der Ausgangssituationen besser geht. Eine solche Abstufung kann durch sogenannte »equity weights « (Deutscher Ethikrat 2012, S. 409) erreicht werden: »Das sind Multiplikationsfaktoren, die bewirken, dass Zugewinne am unteren Ende der QALY-Skala [...] bei der indikationsübergreifend vergleichenden Bewertung von Therapien stärker ins Gewicht fallen als Zugewinne am oberen Ende der Skala [...]« (Deutscher Ethikrat 2012, S. 409). Zudem spiegelt sich im Hinblick auf die Handhabung von Notfallbehandlungen bzw. dringenden Behandlungen die allgemeine Frage nach dem Anspruch bzw. den Rechten der Bürger (Versicherten) auf medizinische Versorgung wider: so könnte die Forderung einer medizinischen Minimalversorgung etwa darin bestehen, das Überleben sicherzustellen. Die zu untersuchenden Evaluationsverfahren sind daher hinsichtlich ihrer Berücksichtigung von Krankheitsschweregraden zu bewerten.

9 | Vgl. für eine ähnliches Rechenbeispiel: Deutscher Ethikrat 2012, S. 409. 


\subsubsection{Ausrichtung an 'perfekter Gesundheit،}

Die Dimension Ausrichtung an >perfekter Gesundheit< kann speziell bezogen auf das Verfahren der healthy year equivalents (HYEs) verdeutlicht werden. Darin wird ein Zustand mit einer gegebenen Krankheit vom Patienten ins Verhältnis gesetzt zu einer gewissen Anzahl von Lebensjahren, die er in perfekter Gesundheit verbringen würde. Beispielsweise soll sich ein Patient mit einem Raucherbein, der aber noch 15 Jahre mit amputiertem Bein leben würde, überlegen, wieviele Jahre in vollständiger (= >perfekter $<$ ) Gesundheit er dagegen >eintauschen (=gleichsetzen) würde (vgl. Mehrez und Gafni 1991, S. 141). Bei den HYEs wird daher nach der Anzahl der Jahre bei bester (perfekter) Gesundheit gesucht, die das Äquivalent zur gegebenen Situation darstellen (vgl. Mehrez und Gafni 1991).

$\mathrm{Zu}$ fragen ist hier nach dem jeweiligen Verständnis von »perfekter Gesundheit«. Die Verbindung zu Modellen der Bewertung von Gesundheit entweder im Sinne einer physiologischen, psychischen und anatomischen Gesamtintaktheit oder im Sinne unterschiedlicher Modelle der Lebensqualität (siehe Dimension 5 und den Beitrag von Reinhold Kilian in diesem Band) liegt auf der Hand. Die Idee der »perfekten Gesundheit« konzentriert sich daher in dieser Bewertungsdimension auf diejenige medizinische Perspektive, die versucht, Gesundheitsdaten anhand objektiver Parameter der Unversehrtheit zu erheben. Probleme liegen in a) der Abbildung von vollständiger Gesundheit, b) der Differenzierung kultureller Gesundheitsverständnisse, nach der ein universelles Bild von perfekter Gesundheit nicht existiert und c) interindividuellen temporalen Verschiebungen des Gesundheitsbildes (zum Beispiel im Rahmen eines altersassoziierten »response shift« (vgl. u.a. Schwartz et al. 2007), siehe auch die Dimensionen 4 und 5).

\subsubsection{Lebensdauer}

Bei den QALYs wird als relevante Zielgröße die (Rest-)Lebensdauer explizit berücksichtigt und verrechnet (vgl. Schöffski und Greiner 2008, S. 96). Auch bei den HYEs und den SAVEs wird die verbleibende Lebenszeit berücksichtigt, allerdings in weniger expliziter 
und offensichtlicher Form. So wird bei den HYEs zum Beispiel die Restlebensdauer in der individuellen Nutzenfunktion berücksichtigt (vgl. Mehrez und Gafni 1991, S. 140). In der Dimension >Lebensdauer ist entsprechend auch die Frage nach der Berücksichtigung von älteren vs. jüngeren Patienten zu verorten (vgl. Deutscher Ethikrat 2012, S. 409). Gegenüber dem QALY-Bewertungsinstrument äußert beispielsweise Meran den Kritikpunkt, dass QALYs besonders alte Menschen diskriminerten, »weil ein Jüngerer immer eine höhere Lebenserwartung hat und somit mehr QALYs erwarten kann« (Meran 1994, S. 74; vgl. auch Schurz 1996, S. 38; Deutscher Ethikrat 2012, S. 409). Dies erscheint vor dem Hintergrund einer allgemeinen Konzeption der Menschenwürde, die das Gebot der Nicht-Diskriminierung nach Alter, Geschlecht, religiöser Ausrichtung usw. beinhaltet, durchaus problematisch. Auch in dieser Dimension wäre dann entsprechend zu prüfen, inwiefern die betrachteten Evaluationsverfahren den Aspekt der Lebensdauer relativ und absolut miteinbeziehen.

\subsubsection{Lebensqualität}

Die >Lebensqualität< wird anhand von verschiedenen Erhebungsmethoden ermittelt; dadurch kann in unterschiedlicher Weise gemessen werden, wie verschiedene Zustände bewertet werden (vgl. Schöffski 2008a, S. 326). Die Bewertung hängt u.a. von der Wahl der Erhebungsmethode ab, aber auch von der befragten Person. Daher kann es zu Verschiebungen bzw. Verzerrungen kommen. Die Bezugnahme auf eine meßbare Form der »Lebensqualität« ist umstritten. So weisen verschiedene Autoren auf Probleme und Herausforderungen in Bezug auf Lebensqualitäts-Konzepte hin (vgl. zum Beispiel Schöffski 2008a und Schöffski 2008b; Meran 1994). Für Meran beispielsweise stellt die »Eigenschaft des Menschen, kompensatorische Gegengewichte zu Einschränkungen jeglicher Art entwickeln zu können« (Meran 1994, S. 68) 
ein Meß-Problem für den Lebensqualitäts-Ansatz dar. ${ }^{10}$ Allein deshalb korreliere »Lebensqualität nicht einfach linear mit physischem, sozialem, psychischem und spirituellem Wohlbefinden, sondern kann auch und gerade Ausgleichsmöglichkeiten und Bewältigungsstrategien als Kompensation zur Krankheit mit einbeziehen« (Meran 1994, S. 68). Bei den HYEs wiederum erfolgt die Berücksichtigung der Lebensqualität innerhalb der individuellen Nutzenfunktion (vgl. Mehrez und Gafni 1991, S. 140). Anhand der Beschreibungsdimension >Lebensqualität wäre folglich zu klären, a) ob und in welchem Maße ein zu untersuchendes Messverfahren die Lebensqualität berücksichtigt, b) in welchem Umfang und wie Lebensqualität jeweils definiert wird sowie c) welche Aspekte, die mit dem Wohlbefinden zusammenhängen, nicht berücksichtigt werden.

\section{Berücksichtigung des Selbstverschuldungsgrades einer Krankheit}

In die Bewertung und Priorisierung von Gesundheitsleistungen durch die allgemeine Bevölkerung und damit in die intuitiven Reaktionsmuster der Menschen scheint auch die Ätiologie, die Genese von Krankheitszuständen in (indirekter) Weise einzufließen. Während gesundheitsökonomische Bewertungsverfahren meist nicht berücksichtigen, ob ein Krankheitszustand selbst- oder fremdverschuldet ist und daher auch diese Differenzierung nicht explizit zu beinhalten scheinen, zeigen Untersuchungen von beispielsweise Schomerus et al. (2006) zur Bewertung psychischer Erkrankungen, dass bei der Allokation von Gesundheitsleistungen die Bereitschaft zur Zu- und Verteilung von Ressourcen stark davon abhängt, ob die zu diagnostizierenden oder zu behandelnden Krankheiten vom Patienten selbst verschuldet sind (etwa durch starken Alkohol- oder Tabakkonsum), oder ob es sich um Krankheiten handelt,

10 | Zu der Anpassungsfähigkeit und den daraus resultierenden Herausforderungen bei der Messung von Lebensqualität vgl. auch den Beitrag von Reinhold Kilian in diesem Band. 
die nicht in eine direkte Korrelation zum Verhalten des Patienten gebracht werden können (zu verstehen als nicht selbstverschuldet bzw. >fremdverschuldet<) (vgl. Schmoreus et al. 2006). Hier spielt folglich der Wert der wahrgenommenen Eigenverantwortlichkeit gegenüber dem eigenen Leben/der eigenen Gesundheit eine zusätzliche zentrale Rolle bei der Frage nach der Zuteilung von Gesundheitsleistungen. In der Bevölkerung scheint die Akzeptanz für Ausgaben von fremdverschuldeten Krankheiten größer zu sein als die Akzeptanz für Ausgaben von selbstverschuldeten Krankheiten (vgl. Schomerus et al. 2006). Auch der Wunsch nach sozialer Distanz zu mit sozialer Devianz assoziierten Krankheiten wie zum Beispiel Alkoholismus oder Schizophrenie führt dazu, dass Menschen weniger stark bereit zu sein scheinen, Geld für die Therapie solcher Krankheiten aufzubringen (vgl. Schomerus et al. 2006). Das heißt, mit einer antizipierten hohen persönlichen Verantwortlichkeit für eine Krankheit korreliert negativ die sallgemeine< Bereitschaft, Geld für Gesundheitsleistungen (auf solidarischer Basis) bereitzustellen.

Hier ist aus ethischer Sicht zu hinterfragen, ob und wann tatsächlich eine solche antizipierte Verantwortlichkeit und Kausalität für die eigene Gesundheit eine Mittelkürzung rechtfertigt. So könnte man wie Georg Marckmann einwenden, dass auch bei Personen, die Sportarten mit hohen Risikofaktoren ausüben (etwa Klettern, Motorradfahren) gleichfalls die Mittel für Gesundheitsleistungen gekürzt werden müssten (was zum Teil bereits in einigen Krankenkassen so gehandhabt wird) (vgl. ähnlich Marckmann 2006). Die ethisch brisante Frage betrifft hier wie so oft die nach der Grenzziehung der Anerkennung: Wann fängt ein selbstverschuldeter Zustand an, und wo beginnt möglicherweise ein Zustand, der nicht selbstverschuldet ist? Die graduellen Grenzziehungen erscheinen willkürlich bzw. individuell aushandelbar was eine gesamtgesellschaftliche Bewertung anhand von gesundheitsökonomischen Bewertungsverfahren erneut erschwert oder gar unmöglich macht. 
In Bezug auf die Dimension >Selbstverschuldungsgrad einer Krankheit< wäre zum einen zu prüfen, ob und inwiefern intuitive Reaktionsmuster bzgl. der Bereitschaft zur Kürzung bzw. Gewährung von Gesundheitsleistungen in den gängigen gesundheitsökonomischen Bewertungsverfahren berücksichtigt werden. Gleichfalls wäre zum anderen zu untersuchen, ob auf politischer Ebene errechnete Allokationen und Prioritätenlisten auf Unverständnis und Nicht-Akzeptanz in der Bevölkerung stoßen könnten. Zudem gilt es aber ferner auch zu verhandeln, ob und inwiefern solche intuitiven Bewertungen überhaupt in Entscheidungen zu Leistungsbegrenzungen einfließen sollten. Das heißt, die hier zu klärende, ethisch äußerst komplexe Frage wäre folglich, ob eine Differenzierung in selbst- und nicht-selbst-verschuldete Krankheiten gewünscht ist und inwiefern diese Berücksichtigung der Geneseform von Krankheiten in die Zuteilungsdebatte von Gesundheitsleistungen einfließen sollte.

\section{Chancengleichheit}

Eine weitere wichtige, allgemein zu berücksichtigende Zielgröße bei der Genese von Entscheidungen im Kontext der Leistungsbegrenzung im Gesundheitswesen stellt das Prinzip der Chancengleichheit dar (siehe auch den einleitenden Beitrag von Heiner Fangerau und Kathrin Dengler sowie die Beiträge von Christian Lenk und Walter Pfannkuche in diesem Band). So gibt es Haltungen, die es als wichtig erachten, dass alle Menschen zumindest einen Grundstamm an gleichen Ausgangsbedingungen - Chancen - erhalten sollten (man denke etwa an die Gerechtigkeitskonzeption von Amartya Sen oder an den Ansatz von Norman Daniels, vgl. Sen 2002, Daniels 1985). Man muss dabei nicht so weit gehen, für alle Menschen immer das Gleiche zu fordern. Vielmehr zielt der Ansatz der Chancengleichheit im Allgemeinen darauf ab, allen Menschen prinzipiell die Möglichkeiten zu offerieren, die sie benötigen, um ein in ihren Augen glückliches, gesundes Leben führen zu können. Gerade die Frage nach der Gesundheit spielt hier eine entscheidende Rolle. 
Allerdings besteht die Schwierigkeit dieses Ansatzes darin, diesen Grundstock an gleichen Ausgangschancen zu benennen und einen diesbezüglichen Konsens zu finden. In Bezug auf Gesundheitsleistungen variieren die Ansichten in den aktuellen politischen Debatten, was zum Teil auf den inzwischen schon sprichwörtlichen Wertepluralismus der westlichen Industrienationen zurückzuführen ist, zum Teil aber auch kulturellen und historischen Bedingtheiten geschuldet zu sein scheint (vgl. die Übersicht $z u$ verschiedenen internationalen Ansätzen zur Leistungsbewertung und -begrenzung in: Deutscher Ethikrat 2012, S. 387-389). In pluralistischen Gesellschaften ist es mitunter schwierig, sich auf ein Set minimaler und als gerecht empfundener unveräußerlicher Grund-Gesundheitsstandards bzw. weniger abstrakt auf einen klar definierten und konkretisierten »Umfang einer medizinischen Grundversorgung (Marckmann 2003, S. 338) zu einigen. In Bezug auf die Verteilung von Gesundheitsleistungen bzw. den Zugang zu Behandlungsangeboten bedeutet dies, dass es für den Austausch von Gütern - speziell im Kontext des Gutes >Gesundheit «11 - gewisse unverfügbare Bereiche bzw. Grundgüter gibt, die nicht in ein Tauschverhältnis eingebracht werden können und dürfen (vgl. Kersting 2008, S. 30-33). Die Chancen, die sich Menschen für ihr Leben wünschen, können stark differerieren - je nach individuellem Werte-Rahmen. Diese Schwierigkeit überträgt sich entsprechend in gesundheitsökonomische Bewertungsansätze, die eine Verallgemeinerung von Präferenzen und allgemein akzeptierten Ausgangschancen abzubilden versuchen. So können bereits vor der Durchführung von gesundheitsökonomischen Evaluationen erste Probleme zutage treten, etwa dann, wenn Uneinigkeit darüber herrscht, ob die für das Gesundheitssystem geplanten Ressourcen tatsächlich in die Gesundheitsversorgung fließen sollen oder nicht doch besser zur Befriedigung anderer Bedürfnisse und

11 | Vgl. für den besonderen Status des Gutes 'Gesundheit، den Beitrag von Heiner Fangerau und Kathrin Dengler in diesem Band und die dort aufgeführte Literatur. 
Wünsche (etwa Bildung, Altersvorsorge oder das Bereitstellen von Verkehrsinfrastruktur o.ä.) verwendet werden sollten. ${ }^{12}$ Es ist offensichtlich, dass gesundheitsökonomische Evaluationsverfahren zur Beantwortung dieser grundsätzlichen Fragen nicht allzu geeignet erscheinen. So ist anzunehmen, dass gesundheitsökonomische Evaluationsmethoden die Ausformulierung dessen, was unter >Chancen< bzw. >gleichen/gleichwertigen Chancen< im Gesundheitsbereich zu verstehen ist, kaum leisten können. Doch genau diese Präzisierung und ihre gesamtgesellschaftliche Diskussion und Anerkennung sind unerlässlich, will man zu überzeugenden, einheitlich akzeptierten Bewertungen, Zuteilungen sowie potentiellen Begrenzungen von Gesundheitsleistungen in einer Gesellschaft gelangen.

12 | Zum Problem der Ressourcen- bzw. Budgetbestimmung im Gesundheitswesen und den verschiedenen Ebenen der Allokation vgl. exemplarisch die Übersicht von Marckmann 2003 oder siehe auch Deutscher Ethikrat 2012. 


\section{Kollektivnutzenmaximierung zu Lasten einzelner Individue ${ }^{13}$ und zu Lasten von Entscheidungsspielräumen}

Die Möglichkeit, Gesundheitsleistungen in Modellen zu bewerten und zu vergleichen, hat aufgrund der Logik des Modellierens folgende wichtige Grenzen: Jede Übertragung von Einzelfällen in eine Modellstruktur, die auf den Gesamtnutzen fokussiert, verlangt notwendigerweise eine Abstraktion, die mitunter nicht jedem Einzelfall gerecht zu werden vermag. Zudem sind Modelle zur individuellen Nutzenmaximierung immer in einen Kontext großer Unsicherheiten eingebettet (vgl. Gold et al. 1996, S. 3of.). In den gesundheitsökonomischen Bewertungsverfahren wird eine Perspektive eingenom-

13 | Auf diesen Aspekt weist etwa der Deutsche Ethikrat in seiner Stellungnahme hin, wenn er in Bezug auf das QALY-Verfahren schreibt: "Wenngleich die Maximierung des Nutzens für den einzelnen Patienten zweifellos ein hochrangiges Ziel ist, bringt die Maximierung der Nutzensumme auf gesamtgesellschaftlicher Ebene ethische Probleme mit sich. [...] Die auffälligste Komponente der QALYs besteht darin, dass eine interindividuelle Nutzenmaximierung intendiert ist. Ziel ist es, eine möglichst große Zahl an zusätzlichen QALYs zu generieren, wobei diese über Personengrenzen hinweg aggregiert werden können. Bei der übergeordneten Anwendung der Ergebnisse geht es also nicht um die Erreichung der maximalen Zahl von QALYs für einen bestimmten Patienten, sondern - auf das Kollektiv bezogen - um die Optimierung der QALYs entsprechend einem zur Verfügung stehenden finanziellen Budget. Bei dieser Vorgehensweise können Rechte individueller Personen verletzt werden, indem ihr individueller Nutzen gegen den Kollektivnutzen aufgerechnet wird. Damit kann man diese Methode dem Utilitarismus zuordnen. Die Erzielung des größten Glücks für die größte Zahl an Betroffenen gilt als das Leitprinzip der im 18. und 19. Jahrhundert von Jeremy Bentham und James Mill begründeten Ethik des Utilitarismus. Eine Handlung bewertet sich demnach allein nach ihren Folgen; sie ist moralisch richtig, wenn sie den Gesamtnutzen über alle Betroffenen hinweg maximiert." (Deutscher Ethikrat 2012, S. 407) Den folgenden Überlegungen zur achten Dimension liegen diese Ausführungen zugrunde. 
men, die eine Gesellschaft bzw. eine klar definierte Personengruppe umfasst. Diese auf Gruppen und deren Gesamtnutzenmaximierung orientierten Ansätze können allerdings dazu führen, dass nur die Kollektive, nicht aber die individuellen Einzelwünsche und -bedürfnisse berücksichtigt werden (vgl. zur Nichtberücksichtigung der Interessen einzelner in konsequentialistischen Ethikansätzen: Bleisch und Huppenbauer 2011, S. 58). Die Mittelverteilung orientiert sich an der Maximierung der Gesamtsumme (»Kollektivnutzen«, Deutscher Ethikrat 2012, S. 407). Das kann aber zu Konflikten führen: So bildet etwa die Basis von Rawls` gerechtigkeitstheoretischen Überlegungen die Grundannahme, wonach »jeder Mensch eine Unverletzlichkeit« (Höffe 2010, S. 66) besitzt, die es zu schützen und zu achten gilt und die nicht aufgerechnet werden darf. Das heißt, auch wenn durch die Verletzung eines Einzelnen (sehr) vielen anderen geholfen werden könnte (etwa indem ihr Wohlergehen gefördert oder gar maximiert würde), so ist dennoch die Unverletzlichkeitsregel einzuhalten: jeder Einzelne ist zu schützen.

In diesem Zusammenhang ist noch ein weiterer Aspekt zu thematisieren. Denkbar wäre, dass der Einzelne durch die Beurteilung aus der Perspektive eines Kollektivs eventuell individuellen Entscheidungs- und Handlungsspielraum einbüßen kann (vgl. Bleisch und Huppenbauer 2011, S. 58; siehe auch den Beitrag von Sebastian Kessler in diesem Band). Das kann auch bedeuten, dass die individuelle Entscheidungskompetenz durch die errechneten Ergebnisse aus standardisierten gesundheitsökonomischen Bewertungsverfahren teilweise verdrängt werden. ${ }^{14}$ Übertragen auf das zumindest im tradierten medizinethischen Ideal individuell und situativ angelegte Arzt-Patient-Verhältnis bedeutet dies, dass durch die Vorschriften, die aus gesundheitsökonomischen Bewertungsverfahren für die Akteure im Gesundheitswesen resultieren könnten, der Arzt immer

14 | Für einen Ansatz, der die Entscheidungen zur Gesundheitsversorgung bzw. zu den für jede Person erbrachten Gesundheitsleistungen auf die Ebene des Individuums verlagert, vgl. die Ausführungen im Beitrag von Walter Pfannkuche in diesem Band. 
weniger Spielraum für individuelle therapeutische Entscheidungsfindungen erhält. Indem Ressourcen über Bewertungsmodelle, die sich am Kollektivnutzen orientieren, zugeteilt werden, könnten wichtige und für das individuelle Arzt-Patienten-Verhältnis elementare therapeutische Entscheidungsspielräume (und auch das Vertrauensverhältnis ${ }^{15}$ ) mitunter subtil aberkannt werden. ${ }^{16}$ Dies lässt sich aber auch umgekehrt auslegen: so könnte man argumentieren, dass der Arzt Entlastung finden könnte, weil ihm der Handlungsrahmen durch gesundheitsökonomische Evaluationen und daraus resultierende Entscheidungen vorgegeben wird - und er nicht mehr selber entscheiden muss, wie er die knappen Ressourcen unter seinen Patienten verteilt (Marckmann 2003, S. 341).

So wäre folglich zu klären, für welche Entscheidungssituationen und Kontexte welche Art von Evaluationsverfahren herangezogen werden sollte (vgl. Schmidt-Wilke 2004) und bezüglich der Einengung von Entscheidungsspielräumen ist zudem zu bestimmen, ob und inwiefern eine Stärkung der auch erfahrungsbasierten Entscheidungskompetenz des Arztes zulasten der Verfolgung starrer Vorgaben aus Evaluationsberechnungen sich ergeben würde bzw. wünschenswert wäre.

15 | So schreibt der Deutsche Ethikrat zur Frage "Ärzte als ,Rationierer??: "Unzumutbar sind Budgetierungen, die Ärzte dazu zwingen, einige inrer Patienten - offen oder verdeckt - schlechter zu behandeln, als es den Standards guter Behandlung entspricht, ohne das die Gesellschaft dies offen verantwortet und transparente Zuteilungskriterien erarbeiten lässt und akzeptiert. Diese Zwänge überfordern Ärzte, führen unter Umständen zu inakzeptablen, insbesondere gleichheitswidrigen Verteilungsmustern und gefährden die Vertrauensgrundlage des Verhältnisses zwischen Arzt und Patient. Das heißt aber nicht im Umkehrschluss, dass Ärzte gegebenenfalls keine Rolle bei der Erarbeitung und Umsetzung von Priorisierungsvorgaben spielen sollen." (Deutscher Ethikrat 2012, S. 391)

16 | Gleichwohl ist anzumerken, dass hier kein strikter Kausalzusammenhang bestehen muss. 


\section{FAZIT}

Zusammenfassend lässt sich festhalten, dass unterschiedliche Methoden der Evaluation von Gesundheitsleistungen die hier geschilderten Bewertungsdimensionen in unterschiedlicher Weise adressieren, wodurch sich Profillinien und »Fingerabdrücke « für einzelne Verfahren ergeben können. Wenn Gesundheit und entsprechende Leistungen zu ihrer Förderung, Wiederherstellung oder Wahrung bewertet werden sollen, so schlagen wir vor, zunächst die sachlich in Frage kommenden Evaluationsinstrumente durch die möglichen Anwender darauf prüfen zu lassen, wie die Instrumente nach Anwendermeinung die hier geschilderten Bewertungsdimensionen adressieren, welche Profillinie sich für welche Instrumente ergibt und welche einer von den Anwendern gewünschten »Ideallinie« am nächsten kommt. Dabei kann die Präferenz für ein Messverfahren, wie Schmidt-Wilke dies anhand ihrer sieben Bewertungskriterien (s.o.) herausgearbeitet hat, je nach Zielfunktion (Informationsverwendung) unterschiedlich ausfallen (Schmidt-Wilke 2004). So ist es auch in Bezug auf die hier vorgestellten acht Beschreibungsdimensionen denkbar, dass sich für verschiedene Kontexte und Informations- bzw. Entscheidungssituationen jeweils unterschiedliche »Ideallinien« herausbilden lassen (vgl. Schmidt-Wilke 2004).

In Bezug auf den Aspekt der Kosten stellt sich die Frage, welchen Stellenwert sie bei der Bewertung von Gesundheitsleistungen einnehmen sollen. So schreibt etwa Weyma Lübbe: »Das Kriterium der Kosteneffektivität sollte nicht als unabhängiger Bestandteil eines multikriterial gewichteten Priorisierungskonzepts eingesetzt werden « (Lübbe Sondervotum 2012, S. 438). Zumindest aus historischer Sicht liegen mit der rassenhygienischen Bewertung von Leistungen Modelle vor, die die Gefahr des Beschreitens einer »schiefen Ebene « benennen, wenn Gesundheit allein auf ein Kollektiv bezogen in vornehmlich ökonomischen Kriterien bemessen wird (vgl. Fangerau 2009 und vor allem Allen 2001). Somit könnte noch - als weitere Beschreibungsdimension - explizit der Kostenaspekt in den Beschreibungsdimensionen aufgegriffen werden. 
Es zeigt sich also, dass die Arbeit an den Beschreibungsdimensionen nicht als abgeschlossen anzusehen ist, sondern eine Weiterentwicklung vielmehr erwünscht und erhofft ist. Somit haben wir erste Schritte für die Erstellung eines Modells zur Erfassung von möglichen Grenzen und Problembereichen von gesundheitsökonomischen Evaluationsverfahren mit der Formulierung von Dimensionen vorgestellt, deren zentralste Teilaspekte in den folgenden Beiträgen eine Vertiefung erfahren.

\section{LITERATUR}

Allen, G.E. (2001): Essays on science and society. Is a new eugenics afoot? Science 294(5540): 59-61.

Bleisch, B.; Huppenbauer, M. (2011): Ethische Entscheidungsfindung. Ein Handbuch für die Praxis. Zürich: Versus Verlag.

Daniels, N. (1985): Just Health Care (Studies in Philosophy and Health Policy). Cambridge: Cambridge University Press.

Deutscher Ethikrat (2012): Nutzen und Kosten im Gesundheitswesen - Zur normativen Funktion ihrer Bewertung. Stellungnahme Januar 2011. In: Honnefelder, L.; Sturma, D.; (in Verbindung mit Beckmann, J.P.; Horn, C.; Siep, L.) (Hrsg): Jahrbuch für Wissenschaft und Ethik 16. Berlin/Boston: de Gruyter, 379-439.

Fangerau, H. (2009): Genetics and the Value of Life: Historical Dimensions. Medicine Studies 1: 105-112.

Gold, M.R.; Siegel, J.; Russell, L.; Weinstein, M. (1996): Cost-Effectiveness in Health and Medicine. Oxford/New York: Oxford University Press.

Höffe, O. (2010): Gerechtigkeit. Eine philosophische Einführung. München: C.H.Beck.

Kersting, W. (2008): Gerechtigkeitsethische Überlegungen zur Gesundheitsversorgung. In: Schöffski, O.; Schulenburg, J.M.v.d. (Hg.): Gesundheitsökonomische Evaluationen. 3. Auflage. Berlin/ Heidelberg: Springer, 23-47. 
Lübbe, W. (2012): Sondervotum (innerhalb der Stellungnahme Januar 2011 des Deutschen Ethikrates »Nutzen und Kosten im Gesundheitswesen - Zur normativen Funktion ihrer Bewertung«). In: Honnefelder, L.; Sturma, D. (in Verbindung mit Beckmann, J.P.; Horn, C.; Siep, L.) (Hrsg): Jahrbuch für Wissenschaft und Ethik 16. Berlin/Boston: de Gruyter, 379-439, hier: 425-439.

Marckmann, G. (2003): Verteilungsgerechtigkeit in der Gesundheitsversorgung. In: Düwell, M.; Steigleder, K. (Hg.): Bioethik. Frankfurt a.M.: Suhrkamp, 333-343.

Marckmann, G. (2006): Verteilungsgerechtigkeit in der Gesundheitsversorgung. In: Schulz, S.; Steigleder, K.; Fangerau, H.; Paul, N. (Hg.): Geschichte, Theorie und Ethik der Medizin. Eine Einführung. Frankfurt a.M.: Suhrkamp, 183-208.

Marckmann, G.; Siebert, U. (2002): Prioritäten in der Gesundheitsversorgung: Was können wir aus dem »Oregon Health Plan« lernen? Deutsche Medizinische Wochenschrift 127 (30): 1601-1604.

Marckmann, G.; Siebert, U. (2011): Nutzenmaximierung in der Gesundheitsversorgung. Eine ethische Problemskizze. In: Gethmann-Siefert, A.; Thiele, F. (Hg.): Ökonomie und Medizinethik. 2. Auflage. München: Wilhelm Fink, 111-139.

Mehrez, A.; Gafni, A. (1991): The Healthy-year equivalents: How to Measure Them Using the Standard Gamble Approach. Medical Decision Making 11(2), 140-146.

Meran, J.G. (1994): Lebensqualität in der Medizin. In: Benzenhöfer, U. (Hg.): Herausforderung Ethik in der Medizin. Beiträge aus der Medizinischen Hochschule Hannover. Frankfurt a.M.: Peter Lang, 63-79.

Nord, E. (1992): An alternative to QALYs: the saved young life equivalent (SAVE). British Medical Journal 305: 875-877.

Nord, E. (1993) Unjustified use of the Quality of Well-Being scale in priority setting in Oregon. Health Policy 24: 45-53.

Schmidt-Wilke, J. (2004): Nutzenmessung im Gesundheitswesen. Analyse der Instrumente vor dem Hintergrund zielfunktionsabhängiger Informationsverwendung. Wiesbaden: Deutscher UniversitätsVerlag/GWV Fachverlage. 
Schöffski, O. (2008): Einführung. In: Schöffski, O.; Schulenburg, J.M.v.d. (Hg.): Gesundheitsökonomische Evaluationen. 3. Auflage. Berlin/Heidelberg: Springer, 3-12.

Schöffski, O. (2008a): Lebensqualität als Ergebnisparameter in gesundheitsökonomischen Studien. In: Schöffski, O.; Schulenburg, J.M.v.d. (Hg.): Gesundheitsökonomische Evaluationen. 3. Auflage. Berlin/Heidelberg: Springer, 321-334.

Schöffski, O. (2008b): Nutzentheoretische Lebensqualitätsmessung. In: Schöffski, O.; Schulenburg, J.M.v.d. (Hg.): Gesundheitsökonomische Evaluationen. 3. Auflage. Berlin/Heidelberg: Springer, 335-385.

Schöffski, O.; Schulenburg, J.M.v.d. (Hg.): Gesundheitsökonomische Evaluationen. 3. Auflage. Berlin/Heidelberg: Springer.

Schomerus, G.; Matschinger, H.; Angermeyer, M.C. (2006): Preferences of the public regarding cutbacks in expenditure for patient care. Social Psychiatry and Psychiatric Epidemiology 41: 369-377.

Schulenburg, J.M.v.d.; Greiner, W.; Jost, F.; Klusen, N.; Kubin, M.; Leidl, R.; Mittendorf, T.; Rebscher, H.; Schöffski, O.; Vauth, C.; Volmer, T.; Wahler, S.; Wasem, J.; Weber, C., und die Mitglieder des Hannoveraner Konsens (2007): Deutsche Empfehlungen zur gesundheitsökonomischen Evaluation - dritte und aktualisierte Fassung des Hannoveraner Konsens. Gesundheitsökonomie a Qualitätsmanagement 12: 285-290.

Schurz, G. (1996): Der Wert des menschlichen Lebens. Ethica 4(1): $23-45$.

Schwartz, C.E.; Andresen, E.M.; Nosek, M.A.; Krahn, G.L., RRTC Expert Panel on Health Status Measurement (2007): Response Shift Theory: Important Implications for Measuring Quality of Life in People With Disability. Archives of Physical Medicine and Rehabilitation 88(4): 529-536.

Sen, A. (2002): Ökonomie für den Menschen: Wege zu Gerechtigkeit und Solidarität in der Marktwirtschaft. München: Deutscher Taschenbuch Verlag. 
Ubel, P.A.; Loewenstein, G.; Scanlon, D.; Kamlet, M. (1996): Individual Utilities Are inconsistent with Rationing Choices: A Partial Explanation of Why Oregon`s Cost-Effectiveness List Failed. Medical Decision Making 16: 108-116. 
Gesundheit und Gerechtigkeit 



\section{Konzeptionen von Gerechtigkeit und der Umgang mit begrenzten Ressourcen}

Christian Lenk

\section{EINFÜHRUNG UND THEORETISCHE VorüberLegungen}

Wer sich mit Verteilungsproblemen und Fragen zur Gerechtigkeit beschäftigt, sieht sich zunächst mit dem Problem konfrontiert, dass unterschiedliche, letztlich miteinander konkurrierende Ansätze in Ethik und politischer Philosophie existieren. Im Bereich der Gerechtigkeit stehen sich in der internationalen Diskussion heute zwei sehr einflussreiche Ansätze gegenüber: einmal John Rawls' Theorie der Gerechtigkeit von 1971, die vom Autor in den nachfolgenden Jahrzehnten immer wieder weiterentwickelt wurde. Im Gefolge und in der kritischen Auseinandersetzung mit Rawls wurden eine Reihe alternativer Ansätze entwickelt, von denen sich insbesondere Michael Walzers Ansatz der Sphären der Gerechtigkeit in den letzten Jahren als einflussreich herauskristallisiert hat. Während sich der Rawls'sche Ansatz durch ausführliche Überlegungen zur normativen Begründung von Gerechtigkeit sowie zur gesellschaftlichen Grundstruktur der gerechten Gesellschaft auszeichnet, liegt der Schwerpunkt bei Walzer auf unterschiedlichen Kontexten (»Sphären«) der Gerechtigkeit sowie der Frage, wie sich die normative Logik der einzelnen Sphären voneinander unterscheidet.

Hinsichtlich der Möglichkeit einer Lösung konkreter Verteilungsprobleme, wie sie uns in dem vorliegenden Beitrag interessieren werden, erscheint der Ansatz von Rawls jedoch als defizitär, 
da er stark auf gerechtigkeitstheoretische Grundlagen fokussiert, während konkrete Probleme der Gerechtigkeit in den Hintergrund treten. Da dies eine direkte Folge des Theoriedesigns ist, ist es wenig verwunderlich, dass es auf der Anwendungsebene zu Defiziten kommt und wenig über die Bewertung konkreter Handlungen und Verteilungskonstellationen gesagt werden kann.

Eine Ausnahme stellen in dieser Hinsicht etwa die Arbeiten von Norman Daniels dar, der für den Bereich der Gerechtigkeit im Gesundheitswesen den Rawls'schen Ansatz in Richtung einer angewandten Ethik weiterentwickelt hat. ${ }^{1}$ Insbesondere in seiner Monographie Just Health Care aus dem Jahr 1985 vertritt Daniels dabei den Standpunkt, dass Rawls' grundlegende Prinzipien der Gerechtigkeit (Rawls 1999, Kap. 2, 11, S. 52 ff.) in die Institutionen des Gesundheitswesens eingebracht werden müssen. Wenn man davon ausgeht, dass diese Prinzipien bei Rawls für die gesamte Gesellschaft gelten sollen, stellt sich allerdings die Frage, unter welchen Bedingungen dies möglich ist. Weiterhin wurde Rawls` Theory of Justice ausdrücklich als eine ideale Theorie für eine wohlgeordnete Gesellschaft konzipiert (Rawls 1999, S. 7f., Kap. 8, 69, S. 397ff.). Es bleibt jedoch nach wie vor ein ungelöstes Problem, wie die Vorgaben dieser Theorie unter nicht-idealen Umständen umgesetzt werden können.

\section{Pluralismus der Prinzipien der Gerechtigkeit IN DER "NICHT-IDEALEN GESELLSCHAFT॥?}

Charakteristisch für Ansätze, die auf konkrete Verteilungsprobleme fokussieren, ist jeweils, dass sie unterschiedliche Kontexte oder Sphären (bei Walzer z.B. Sicherheit und Wohlfahrt, Geld und Waren, Arbeit, Erziehung und Bildung usw.) unterscheiden (siehe Abbildung 1), in denen bestimmte Regeln der Gerechtigkeit relevant sind. In diesem Zusammenhang kann man auch von einem »Kontextua-

1 | Vgl. dazu auch den aktuellen Beitrag von Birnbacher 2012. 
lismus der Gerechtigkeit« sprechen. ${ }^{2}$ Die Existenz dieser verschiedenen Sphären besitzt selbst einen normativen Hintergrund, wie Stefan Gosepath unter Verweis auf David Miller (1995) darlegt: »Wenn alles käuflich oder alles eine Frage der Macht ist, [...] [haben] die bereichsspezifischen Verteilungsregeln [...] ihre Zuständigkeit überschritten. Der Übergriff ist zu unterbinden, sonst wird besonders evident, daß Personen nicht als Gleiche geachtet werden« (Gosepath 2004, S. 283).

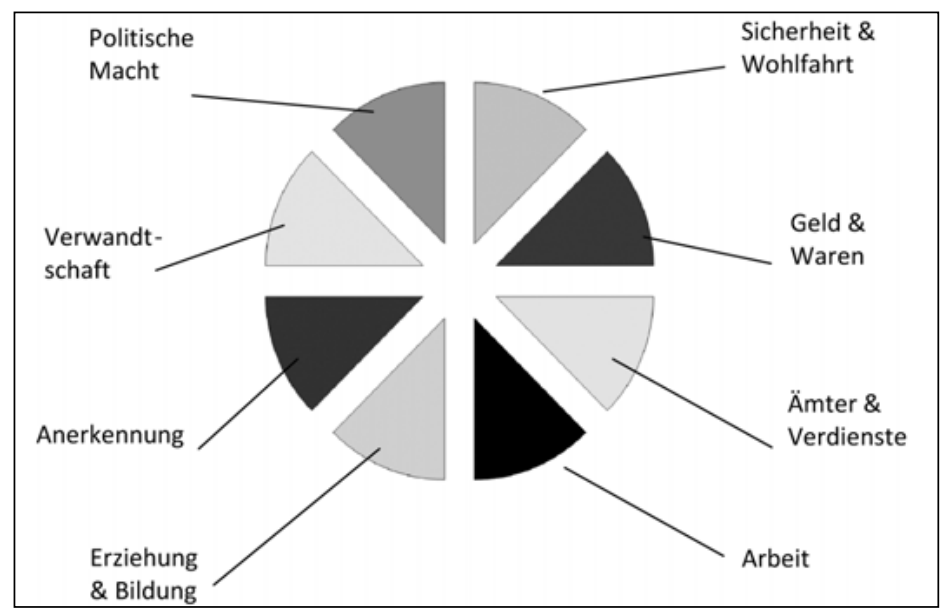

Abbildung 1: „Sphären“ der Gerechtigkeit (Walzer/modifizierte Darstellung durch den Autor)

Wie Walzer argumentiert, ist in Bezug auf soziale Gerechtigkeit jedoch nicht der Charakter der Dinge als individueller Besitz oder als Wertgegenstand von Bedeutung, sondern als soziales Gut, d.h. insofern diese Güter eine allgemein anerkannte gesellschaftliche Relevanz haben (Walzer 2006, S. 32). Die persönlichen Präferenzen, die der Einzelne hat, werden überlagert von der gesellschaftlichen Be-

2 | Dabei gilt es jedoch, wie bereits Rainer Forst (Forst 1996, S. 10) formuliert, einen Ansatz zur Gerechtigkeit zu formulieren, "der den Vorwurf der Kontextblindheit ebenso vermeidet wie einen Kontextualismus, der den universalistischen Kern der Forderung nach 'Gerechtigkeit، verkennt." 
deutung von Gütern wie etwa Nahrungsmitteln, Leistungen im Gesundheitswesen, ökonomischen Ressourcen, Zugang zu Bildungsgütern usw. Es ist nun eine wesentliche Beobachtung, dass diese Güter, gerade weil und insofern sie soziale Güter darstellen, in ihren Bereichen unterschiedlichen Verteilungslogiken unterliegen. Wie Walzer es ausdrückt, konstituiert »[d]as je einzelne soziale Gut oder Set von Gütern [...] gewissermaßen seine eigene Distributionssphäre, innerhalb deren sich nur ganz bestimmte Kriterien und Arrangements als angemessen und dienlich erweisen«(Walzer 2006, S. 36).

So werden etwa lebensnotwendige Güter wie Nahrung, Wasser und medizinische Versorgung im Katastrophenfall nach Bedarf zugeteilt, während ein spezialisierter Arbeitsplatz an die Bewerberin oder den Bewerber vergeben werden soll, der die geforderte Qualifikation aufweist. Wenn diese Verteilungen in dem genannten Modus durchgeführt werden, nennen wir sie nicht nur (wie Walzer) »angemessen und dienlich« (ebd.), sondern »gerecht«. Wenn aber diese Verteilungsmodi verletzt werden, wenn die lebensnotwendigen Güter im Katastrophenfall z.B. nicht den Bedürftigsten zukommen, sondern auf dem freien Markt an den Meistbietenden verkauft werden, so empfinden wir dies als einen Verstoß gegen die gerechte Vorgehensweise. An anderer Stelle spricht Walzer auch davon, dass die Grenzen dieser Sphären gegen Übergriffe aus anderen Bereichen (z.B. gegen wirtschaftliche Gesichtspunkte) »verteidigt« werden müssen (Walzer 2006, S. 327). Die Aufrechterhaltung des gerechten Zustandes folgt also nicht allein aus der Zuordnung eines Verteilungsmodus zu einem bestimmten Gut, sondern bedarf des aktiven Handelns, um eine als gerecht verstandene normative Ordnung zu bewahren.

Walzer's Rede von den »Kriterien und Arrangements« (s.o.) meidet die Sprache der Prinzipienethik und Deontologie. Doch wäre es überhaupt möglich, Walzers Ansatz in ein prinzipienethisches Kalkül zu übertragen? Diesen Versuch hat der Autor dieses Textes in der folgenden Tabelle (1) unternommen. Sicherlich bedürfte es, um die Tragweite und Produktivität eines solchen Ansatzes abschätzen zu können, einer weitaus ausführlicheren Behandlung sowie einer zusätzlichen theoretischen Fundierung, als sie an dieser Stelle möglich 
ist. Doch immerhin zeigt die Übersicht und Zuordnung von Prinzipien zu einzelnen Bereichen, dass ein derartiger prinzipienethischer Ansatz immerhin möglich ist und einige Plausibilität besitzt.

\begin{tabular}{|c|c|c|}
\hline Art des Prinzips & Anwendungsbereich & Beispiele \\
\hline $\begin{array}{l}\text { Egalitäres } \\
\text { Verteilungsprinzip }\end{array}$ & $\begin{array}{l}\text { Güter, die allen } \\
\text { Menschen oder allen } \\
\text { Mitgliedern einer Ge- } \\
\text { meinschaft zukom- } \\
\text { men sollen }\end{array}$ & $\begin{array}{l}\text { Bildung; Chancen- } \\
\text { gleichheit im Zugang } \\
\text { zu öffentlichen Äm- } \\
\text { tern und Positionen; } \\
\text { Grundrechte; bedin- } \\
\text { gungsloses Grundein- } \\
\text { kommen }\end{array}$ \\
\hline $\begin{array}{l}\text { Ausgleichende } \\
\text { Gerechtigkeit }\end{array}$ & $\begin{array}{l}\text { Gericht; gesellschaft- } \\
\text { liche oder natürliche } \\
\text { Ungleichheiten }\end{array}$ & $\begin{array}{l}\text { Kompensation eines } \\
\text { entstandenen Scha- } \\
\text { dens; Ausgleich sozia- } \\
\text { ler Benachteiligung }\end{array}$ \\
\hline Bedarfsprinzip & $\begin{array}{l}\text { Elementare Bedürf- } \\
\text { nisse }\end{array}$ & $\begin{array}{l}\text { Ressourcen im } \\
\text { Gesundheitswesen; } \\
\text { Verteilung von Res- } \\
\text { sourcen im Katastro- } \\
\text { phenfall }\end{array}$ \\
\hline Tauschgerechtigkeit & $\begin{array}{l}\text { Markt; gesellschaft- } \\
\text { liche/ökonomische } \\
\text { Kooperation }\end{array}$ & $\begin{array}{l}\text { Freiwilliger Tausch } \\
\text { oder Kauf von Gütern } \\
\text { in der Marktwirt- } \\
\text { schaft }\end{array}$ \\
\hline $\begin{array}{l}\text { Verdienstprinzip/ } \\
\text { Leistungsprinzip }\end{array}$ & $\begin{array}{l}\text { Güter oder Positionen, } \\
\text { die aufgrund beson- } \\
\text { derer Leistungen oder } \\
\text { Verdienste verteilt } \\
\text { werden sollen }\end{array}$ & $\begin{array}{l}\text { Ämter und Positionen } \\
\text { im Staat oder in pri- } \\
\text { vaten Organisationen; } \\
\text { finanzielle Gratifika- } \\
\text { tionen; Löhne }\end{array}$ \\
\hline $\begin{array}{l}\text { Nützlichkeitsprin- } \\
\text { zip/»Das größte Glück } \\
\text { der größten Zahl“ }\end{array}$ & $\begin{array}{l}\text { Knappe Güter sollten } \\
\text { mit maximalem } \\
\text { Nutzen für die Gesell- } \\
\text { schaft verteilt werden }\end{array}$ & $\begin{array}{l}\text { Priorisierung/Poste- } \\
\text { riorisierung von Gü- } \\
\text { tern und Leistungen }\end{array}$ \\
\hline
\end{tabular}

Tabelle 1: Verschiedene Verteilungsprinzipien mit Anwendungsbeispielen 


\section{Zum Nutzenbegriff in der Medizin}

»Nutzen« ist ein Begriff, der in der Alltagssprache eher unkontrovers verwendet wird, wobei man jedem Sprecher zugesteht, den Nutzenbegriff aus seiner subjektiven Sichtweise zu bestimmen. Ganz anders steht es allerdings in der wissenschaftlichen, ethischen oder politischen Diskussion des Nutzenbegriffs, wenn es darauf ankommt, einen für eine größere Gruppe von Personen oder eine Institution verbindlichen Nutzenbegriff zu definieren. Neben der Problematik des Gegensatzes von subjektiv und objektiv zu bestimmendem Nutzen besteht zusätzlich die Schwierigkeit, dass sich verschiedene Disziplinen oder Subdisziplinen auf verschiedene Nutzenbegriffe beziehen. So gibt es Überlegungen zum Nutzen in der Ethik (z.B. im Utilitarismus oder im Rahmen des Benefizienzprinzips), in der Ökonomie und ihren Subdisziplinen (von betriebswirtschaftlicher oder volkswirtschaftlicher Seite) ebenso wie in der Medizin und Gesundheitsökonomie, wobei jeweils unterschiedliche Definitionen zugrunde gelegt werden. Für die vorliegende Untersuchung möchte ich mich auf vier Aspekte des Nutzenbegriffs beschränken, nämlich (a) den individuellen Nutzen, (b) den ökonomischen Nutzen, (c) den Gebrauch von QALYs ${ }^{3}$ sowie (d) den gesellschaftlich agglomerierten Nutzen, und zwar jeweils bezogen auf den Bereich der Medizin.

\section{a) Individueller Nutzen}

Innerhalb des medizinischen Systems kann man im Allgemeinen davon ausgehen, dass bei den meisten Behandlungen ein gewisser Konsens zwischen Arzt und Patient besteht, worin der Nutzen einer medizinischen Behandlung liegt. So formuliert die Musterberufsordnung (BÄK 2011), es sei die »Aufgabe der Ärztinnen und Ärzte

3 | VgI. hierzu auch die Ausführungen zum QALY-Konzept und anderen gesundheitsökonomischen Nutzen-Definitionen im Beitrag von Kathrin Dengler in diesem Band. 
[...], das Leben zu erhalten, die Gesundheit zu schützen und wiederherzustellen, Leiden zu lindern [und] Sterbenden Beistand zu leisten [...].« Wenn dies die Kernaufgaben der Ärzte sind, so lässt sich andersherum folgern, dass sich in der Erfüllung dieser Aufgaben wohl auch der wesentliche individuelle Nutzen ergibt, den die Patienten bei den Ärzten suchen.

Im Vorgriff auf die anderen Nutzenbegriffe (ökonomischer Nutzen etc.) können wir jedenfalls schon festhalten, dass der individuelle Nutzen der Patienten normalerweise strikt auf die individuelle Gesundheit bezogen ist. In anderen Konstellationen der Gesundheitsversorgung, insbesondere in anderen Gesundheitssystemen, werden sich die Patienten zusätzlich fragen, ob es ihrem ökonomischen Nutzen entspricht, eine bestimmte Behandlung durchführen zu lassen oder eine bestimmte Form der Versicherung zu wählen. ${ }^{4}$ Da die Gesetzliche Krankenversicherung (GKV) in Deutschland einerseits obligatorisch und in ihrem Umfang weitgehend festgelegt ist, die Versicherten andererseits auch nicht erfahren, wie teuer ihre Behandlungen sind, ist die Frage nach dem individuellen, ökonomischen Nutzen der GKV in Deutschland für den einzelnen Versicherten sinnlos. Selbst wenn er erfahren würde, dass er z.B. im Laufe eines Jahres 4.000 Euro mehr in die GKV einzahlt, als für seine Behandlungen ausgegeben wurde, so folgen daraus keine möglichen Konsequenzen (außer unter bestimmten Bedingungen der Wechsel in die Private Krankenversicherung).

Allerdings kann die individuelle Vorstellung des Patienten vom Nutzen einer Behandlung verschieden sein von der des Arztes oder dem Nutzen, der durch das Sozialrecht oder die zuständigen Gremien festgelegt wird. Auch können verschiedene Patienten einem vergleichbaren Eingriff einen unterschiedlichen, individuellen Nutzen zuordnen. Ein klassischer Fall ist die Therapie am Lebensende, wobei der Schwerpunkt einerseits auf Lebensverlängerung (als klassische ärztliche Aufgabe), andererseits auf die Bewahrung einer

4 | Vgl. hierzu die Ausführungen von Walter Pfannkuche in diesem Band. 
möglichst hohen Lebensqualität gelegt werden kann. Der Fall des Child $B$ aus Großbritannien ist ein ähnlich gelagerter Fall unter umgekehrten Vorzeichen, in dem bei einem an Leukämie erkrankten Kind (Entwistle et al. 1996) die zuständigen Behörden die Kostenübernahme für die weitere Behandlung ablehnten, die Familie der jungen Patientin die Behandlung aber weiterführen wollte.

Weitere Bereiche, in denen der individuelle Nutzen von der vorgesehenen Form der Behandlung abweicht, sind seltene Erkrankungen, innovative Behandlungen, die noch nicht von Versicherungen abgedeckt werden, sowie nicht medizinisch indizierte Behandlungen. Ein Beispiel aus dem letzten Bereich, das auch Eingang in die ethische und rechtliche Diskussion gefunden hat (Markus 2006), ist der medizinisch nicht indizierte Kaiserschnitt. Dabei kann der gesuchte Nutzen für die nicht medizinisch indizierte Sectio ebenso in der Vermeidung der Schmerzen der natürlichen Geburt liegen wie in (sub-)kulturell determinierten Vorstellungen des »schönen« Frauenkörpers.

Während wir im Normalfall der medizinischen Behandlung davon ausgehen, dass zwischen Arzt und Patient ein Konsens über das $\mathrm{zu}$ erreichende Behandlungsziel und damit den zu verwirklichenden Nutzen besteht, gibt es also eine Reihe von Bereichen, in denen individueller und allgemein definierter Nutzen auseinanderfallen. Wenn dies der Fall ist, wird das gewöhnlich dazu führen, dass eine medizinische Behandlung nicht von der Krankenversicherung bezahlt wird. Eventuell besteht dann die Möglichkeit, die Behandlung als Privatleistung erbringen zu lassen. Eine Implikation der Ressourcenknappheit ist also, dass der »nur« individuelle Nutzen einer Behandlung im allgemeinen nicht als hinreichend angesehen wird, um die Kosten für eine Behandlung aus der öffentlichen Krankenversicherung erstatten zu lassen, und dass ein allgemein anerkannter Nutzen nachgewiesen werden muss. Bei schweren Erkrankungen, die evtl. mit einer innovativen Behandlung therapiert werden könnten bzw. bei Fragen der Therapiebegrenzung wie im Fall des Child B kann die dahinter stehende Problematik durchaus existenzielle Konsequenzen nach sich ziehen. 


\section{b) Ökonomischer Nutzen}

Dem klassischen Verständnis nach ist »Ökonomie zunächst einmal die Kunst des guten Haushaltens, also des optimalen Einsatzes von knappen Mitteln zur Erreichung bestimmter Ziele« (Rüegger et al. 2012, S. 155). Umgangssprachlich wird der ökonomische Nutzen jedoch in erster Linie mit der Erwirtschaftung eines finanziellen Gewinns verbunden. Der ökonomische Nutzen kann nun einerseits darin bestehen, eine bestimmte Leistung mit geringem Ressourcenaufwand zu erbringen, andererseits darin, einen Gewinn aus ökonomischer Tätigkeit zu ziehen und zu maximieren. Das Verhältnis des Patienten zur Frage des ökonomischen Nutzens muss in einem marktwirtschaftlich organisierten Gesundheitssystem stark von der Bedeutung dieser Frage in einem öffentlichen Versicherungssystem divergieren. In einem marktwirtschaftlichen System muss der Patient sich selbst fragen, ob der geforderte Preis dem Nutzen einer zu erbringenden Leistung entspricht, während die Beantwortung dieser Frage in einem regulierten Versicherungssystem in die Institutionen ausgelagert wird. ${ }^{5}$ Da sich der Patient im letztgenannten Modell in vielen Fällen um die Bezahlung einer Behandlung nicht zu kümmern braucht, ist die Frage, die ihn hier interessiert, eher die, ob der erwartete Nutzen die mit der Behandlung verbundenen Belastungen und Risiken rechtfertigt. Durch Zuzahlungsregeln (Praxisgebühr, Gebühr für Krankenhausaufenthalt) versucht man, die Versicherten von der Inanspruchnahme nicht oder nicht ausreichend notwendiger Gesundheitsleistungen abzuhalten. Dies ändert aber nichts an der Tatsache, dass die eigentliche Kaufentscheidung (Entspricht der erwartete Nutzen dem geforderten Preis?) in einem Versicherungssystem letztlich suspendiert ist (wobei man zurecht darauf hingewiesen hat, dass in einer Reihe von Fällen, z.B. bei der Versorgung von Notfällen, das Marktmodell auch inadäquat für die Regulierung des Gesundheitswesens ist).

5 | Dieser Frage nach der Verortung der Entscheidung geht Walter Pfannkuche in seinem Beitrag in diesem Band ausführlich nach. 
Neben dem ökonomischen Nutzen, den sich der Einzelne von einem medizinischen Eingriff verspricht (z.B. die Erhaltung oder Wiederherstellung seiner Arbeitskraft), gibt es auch aus der gesellschaftlichen Perspektive einen hohen oder niedrigen Nutzen, der aus einem medizinischen Eingriff resultieren kann. Dementsprechend hat man die Frage untersucht, ob man diese Art von Nutzen als Kriterium für Allokationskriterien verwenden sollte. So kann in der zeitlichen Perspektive ein junger Mensch stärker z.B. von einer transplantierten Niere profitieren als ein alter Mensch. Und eine teure und lebenserhaltende medizinische Maßnahme erzeugt, bezogen auf die verbleibende Lebensspanne, bei einem jungen Patienten einen höheren Nutzen als bei einem alten Patienten. Solche Überlegungen konfligieren aber offensichtlich mit unseren Vorstellungen von Gerechtigkeit, nach denen junge und alte Menschen denselben Zugang zu medizinischen Behandlungen haben sollten, unabhängig von einer Abschätzung darüber, bei welchem Patienten sich die Behandlung am Besten »rentiert«.

\section{c) QALYs}

Das Konzept der QALYs (Quality Adjusted Life Years) beruht letztlich auf der Idee einer unterschiedlichen Gewichtung von Lebensjahren gemäß der in diesen Jahren vorhandenen Lebensqualität. Ein Jahr bei perfekter Lebensqualität wird demnach mit dem Wert »1« gewichtet, während der Wert »o « das andere Extrem, den Tod des Patienten (keine vorhandene Lebensqualität) wiedergibt. Die zeitweilige Popularität des QALY-Konzeptes speist sich nach Beobachtung des Autors aus zwei verschiedenen Quellen, nämlich einmal dem Anspruch, lebensweltliche Erfahrungen im medizinischen Bereich (gesundheitliche Zustände und die Ergebnisse medizinischer Behandlungen) in einem theoretisch übersichtlichen Konzept handhabbar und anschlussfähig an ökonomische Modelle zu machen. Durch die Anschlussfähigkeit an die Ökonomie werden zugleich Verbindungen zu philosophischen Theorien wie dem Utilitarismus möglich, da ja mit dem QALY-Konzept, zumindest theoretisch, das 
"größte Glück der größten Menge « nicht nur als Ziel postuliert, sondern auch faktisch evaluiert werden kann. ${ }^{6}$ Entscheidungen werden möglich, z.B. welche medizinische Behandlung eher in der Lage ist, mehr QALYs, also letztendlich mehr »Glück« und Wohlbefinden zu produzieren. Da der Utilitarismus auch ein radikaldemokratisches Konzept darstellt (das Wohlbefinden aller Individuen wird gleich gewichtet, es gibt keine Rechtfertigung der Bevorzugung einer Person vor einer anderen), ist er tief in den Ideen der Moderne verwurzelt und damit grundsätzlich attraktiv für sozialreformerische Ansätze.

Die zweite Quelle für die Popularität des QALY-Konzeptes besteht sicherlich in dem Bezug zur »Lebensqualität«, der eine Abwendung von der »Tonnenideologie« der ersten Hälfte des 20. Jahrhunderts darstellte (vgl. Musschenga 1997), nach welcher die Masse wichtiger ist als die Qualität der hergestellten Güter und Dienstleistungen. ${ }^{7}$ Lebensqualität stellt dann einen Oberbegriff dar, in den nicht nur die faktischen Lebensumstände einer Bevölkerung eingehen, sondern auch deren Bewertung durch die betroffenen Individuen. Der Erfolg der »grünen« Parteien in verschiedenen Ländern Europas in den letzten 30 Jahren wird auch als Ergebnis des Umdenkens breiter (meistens eher gebildeter und begüterter) Bevölkerungsteile im Bereich der Lebensqualität im Sinne eines »postmaterialistischen Denkens« gedeutet. Dennoch zeigt gerade das Phänomen der QALYs die Ambivalenz Lebensqualität-geleiteter staatlicher Maßnahmen, da die zugrundeliegende Annahme einer unterschiedlichen Gewichtung von Lebensjahren nur bedingt kompatibel mit denjenigen Verfassungswerten ist, die die Gleichheit aller Bürger vor dem Gesetz vorschreiben. Die Gleichheit muss aber

6 | Zur utilitaristischen Theorie vgl. den Beitrag von Günter Fröhlich in diesem Band.

7 | Der deutsche Begriff der "Tonnenideologie" scheint dabei insbesondere auf die 5-Jahrespläne der sozialistischen DDR und die in diesen ausformulierten ökonomischen Ziele zurückzugehen (vgl. etwa Malycha 2011, S. 30). 
durch einen Ansatz verletzt werden, der unterschiedlichen Lebensjahren einen unterschiedlichen $»$ Wert $\aleph^{8}$ zuordnet.

Auf der einen Seite wird niemand etwas dagegen haben, wenn der Staat versucht, mit allgemeinen Maßnahmen die Lebensqualität seiner Bürger anzuheben. Wenn Lebensqualität aber mit einem Effizienzkalkül verbunden wird (es gibt lohnende und weniger lohnende Investitionen in die Lebensqualität) und an die individuelle Versorgung zurückgebunden ist (ein Patient soll eine medizinische Leistung nicht erhalten, da sich diese für ihn nicht mehr »lohnt«), kommen problematische Implikationen des QALY-Ansatzes zum Vorschein.

Damit soll nicht bestritten werden, dass QALYs in einigen Bereichen durchaus einen sinnvollen Ansatz zum Vergleich z.B. verschiedener Therapieoptionen darstellen können. Sie eignen sich aus den genannten Gründen heraus aber nicht zur globalen Bewertung von Gesundheitsleistungen sowie als alleinige Systematik zur Mittelallokation im Gesundheitswesen. Wie Franz Porzsolt schreibt, muss man sich auch hüten, mit theoretischen Ansätzen Kommensurabilität und Vergleichbarkeit vorzutäuschen, wo diese de facto nicht besteht:

"Für die mehrdimensionale Bewertung stehen verschiedene Konzepte zur Verfügung, z.B. die quality of well being-Skala (QWB), der health utility-Index (HUI) und das Konstrukt der quality adjusted life years (QALYs). Diese Konzepte liefern jedoch keineswegs übereinstimmende Ergebnisse. Es ist unschwer zu erkennen, dass es äußerst problematisch sein kann, z.B. bei Schwerhörigkeit und bei Kniegelenksarthrose, jeweils vergleichbare Schweregrade zu definieren“ (Porzsolt 2011, S. 182).

Alle genannten Ansätze basieren aber letztlich auf der Idee der Möglichkeit einer Vergleichbarkeit verschiedenster Zustände und Krankheitsentitäten. Hier muss möglicherweise noch mehr Arbeit in die bessere Eingrenzung theoretischer Konstrukte auf bestimm-

8 | Zum Wertbegriff vgl. Günter Fröhlich in diesem Band. 
te Anwendungsgebiete (verbunden mit einer Diskussion der daraus entstehenden ethischen Implikationen) investiert werden, da nur so ein sinnvoller Beitrag zur Verteilungsdebatte entstehen kann.

\section{d) Gesellschaftlich agglomerierter Nutzen}

Während aus Sicht des Patienten legitimerweise die Frage nach dem individuellen Vorteil im Vordergrund steht, hat aus Sicht der Organisation des Gesundheitswesens die Zusammenfassung der Gesamtkosten wie des Gesamtnutzens einige Bedeutung. Die Frage ist allerdings, in welche Maßzahl man den Gesamtnutzen eines Gesundheitssystems zusammenfassen kann. Während die Kosten gewöhnlich als prozentualer Anteil am Bruttoinlandsprodukt dargestellt werden (also als Anteil an den insgesamt erzeugten Waren und Dienstleistungen, wobei reichere Gesellschaften dementsprechend in absoluten Zahlen mehr Ressourcen für Gesundheit aufwenden als ärmere Gesellschaften), könnte natürlich theoretisch der Gesamtnutzen durch eine ähnliche Kennzahl wie die der QALYs erfasst werden. Da es sich bei QALYs allerdings in den meisten Fällen um eine relative Erfassung des Nutzens (also eine Behandlung oder Therapie im Vergleich zu einer anderen Therapie) handelt, wäre dann die Frage, an welchem Basiswert sich eine solche Kalkulation orientieren sollte.

De facto hat sich eine andere Bewertungsmethode eingebürgert, nämlich wichtige Kennzahlen verschiedener Gesundheitssysteme wie z.B. die durchschnittliche Lebenserwartung oder die Säuglingssterblichkeit mit den aufgewandten Kosten in Beziehung zu setzen. Dieses Verfahren kann dann für einzelne Versorgungsbereiche detaillierter durchgeführt werden, also z.B. für die Behandlung von Patientinnen mit Brustkrebs oder der Patienten mit kardiovaskulären Erkrankungen. ${ }^{9}$ Diese Vorgehensweise ist weniger standardi-

9 | Dabei wird in einem ersten Schritt die Zahl der geheilten/und oder überlebenden Patientinnen und Patienten in Beziehung zu den entstandenen Kosten gesetzt. Ein Vergleich verschiedener Gesundheitssysteme offenbart 
siert, erlaubt aber aufschlussreiche Vergleiche verschiedener Versorgungsbereiche in unterschiedlichen Ländern, wobei natürlich regionale oder nationale Faktoren, die ebenfalls die Gesundheit beeinflussen, korrigiert werden müssten, um die Effektivität eines Gesundheitswesens bewerten zu können. Interessanterweise ist nun ein System mit starken marktwirtschaftlichen Elementen wie das der USA relativ ineffektiv in einem Vergleich der genannten Kennzahlen (s.u., in Bezug auf die Zahlen aus dem OECD-Bericht 2011). Der Grund dürfte darin bestehen, dass die Zuordnung medizinischer Ressourcen nach dem Vermögen (bzw. dem fehlenden Vermögen) der Patienten im Vergleich zum tatsächlich vorhandenen medizinischen Behandlungsbedarf zur Fehlallokation therapeutischer Ressourcen führt, wie sie z.B. auch für das deutsche System beschrieben wurde.

D.h. den Schwerkranken und stark Behandlungsbedürftigen werden in einem ineffektiven System signifikant weniger Ressourcen zugeordnet, als für eine erfolgreiche Behandlung notwendig wäre, während für die Behandlung der nur leicht Erkrankten signifikant mehr Ressourcen aufgewandt werden, als für die erfolgreiche Behandlung eigentlich notwendig gewesen wäre (z.B. durch Facharzt- oder Chefarztbehandlung, Einsatz teurer diagnostischer Methoden etc.). Im Ergebnis operiert dann ein System wie der britische National Health Service (NHS) als der Prototyp eines staatlich regulierten Gesundheitssystems erfolgreicher als z.B. das U.S.-amerikanische System.

Laut dem OECD-Report Health at a Glance in der letzten Fassung von 2011 gaben z.B. die USA insgesamt 17,4 \% ihres Bruttoinlandsproduktes (BIP) oder pro Kopf 7.960 US-Dollar für Gesundheit und medizinische Versorgung aus (OECD 2011, S. 149, 151). Im Vergleich dazu haben die EU-Staaten Griechenland, Italien und Spanien nur 9,6 \% (GR) bzw. 9,5\% (ITA, SP) des BIP bzw. pro Kopf nur 2.724

dann ein günstiges oder ungünstiges Versorgungsprofil in dem jeweiligen Behandlungsbereich. Siehe im Folgenden die unterschiedlichen Beispiele zur allgemeinen Lebenserwartung wie für den Bereich der Kardiologie. 
US-Dollar (GR), 3.137 US-Dollar (ITA) bzw. 3.067 US-Dollar (SP) ausgegeben (ebd.) Die durchschnittliche Lebenserwartung bei Geburt lag in den EU-Staaten jedoch bei 80,3 (GR) und 81,8 (ITA, SPA) Jahren, in den USA hingegen nur bei 78,2 Jahren. Noch deutlicher fällt der Unterschied bei der Kindersterblichkeit ins Auge, die in den Mittelmeerländern bei 3,1 (GR), 3,7 (ITA) und 3,3 (SP) verstorbenen Kinder auf 1.000 Lebendgeburten lag, in den USA hingegen rund doppelt so hoch bei 6,5 Kindern auf 1.000 Lebendgeburten (ebd., S. 37). Unter diesem Gesichtspunkt besteht also die Attraktivität eines staatlich (und »solidarisch«) organisierten Gesundheitssystems u.a. darin, dass eine Fehlallokation von Ressourcen entsprechend der finanziellen Leistungsfähigkeit der Patienten vermieden werden und ein größerer medizinischer Nutzen bei (durch Umverteilung) gleichmäßiger Ressourcenverteilung erzielt werden kann.

Geht man stärker in die Details, so findet man etwa für Deutschland (das für seine gut ausgebaute Herzmedizin bekannt ist), einen internationalen Spitzenwert von 582 Koronarangioplastien ${ }^{10}$ pro 100.000 Einwohner, während z.B. in der Schweiz als partiell vergleichbarem Land lediglich 134 Angioplastien pro 100.000 Einwohner (23\% des deutschen Wertes) durchgeführt werden. Während in Deutschland im Jahr 2009 jedoch im Durchschnitt 6,8 von 100 Patienten nach Krankenhauseinweisung an einem Herzinfarkt verstarben, waren es in der Schweiz nur 4,5 Patienten von 100 (also $66 \%$ des deutschen Wertes), in Dänemark sogar nur durchschnittlich 2,3 von 100 Patienten (ca. $34 \%$ des deutschen Wertes, OECD 2011, S. 108). Ohne an dieser Stelle zu sehr in die Details dieser sicherlich diffizilen und hochkomplexen Materie eindringen zu wollen, ist auf diese Weise also ein Vergleich der allgemeinen Kennzahlen von Gesundheitssystemen sowie auch von einzelnen Bereichen (z.B. Herzerkrankungen) möglich, wobei Schwerpunkte, Erfolge und (an definierten Kriterien bemessene) Defizite einzelner Gesundheitssysteme sichtbar werden. Relativierend muss selbstver-

10 | Koronarangioplastie=Weitung verengter Herzkranzgefäße, z.B. mit der Technik der Ballondilatation. 
ständlich noch hinzugefügt werden, dass ein methodisch einwandfreier Vergleich nur auf der Grundlage gleicher Arbeitsbedingungen der Gesundheitssysteme möglich wäre, d.h. in diesem Fall insbesondere unter der Voraussetzung vergleichbarer Patientenkollektive in den genannten Ländern. Einerseits gibt die OECD-Statistik nun selbst darüber Auskunft, dass diese Vergleichbarkeit nur bedingt gegeben ist, da es unterschiedliche Prävalenzen für Erkrankungen wie Diabetes, Adipositas, Herz-Kreislauf-Erkrankungen etc. gibt. Da allerdings im Wesentlichen die Industrie- und Schwellenländer Mitglieder der OECD sind, lässt sich eine Vergleichbarkeit im weiteren Sinne sicherlich nicht von der Hand weisen.

\section{Allgemeine Überlegungen zum Phänomen der Knappheit}

Zurückgehend auf David Humes Enquiry Concerning the Principles of Morals $(1777,1975)$ werden in der heutigen philosophischen Diskussion gewöhnlich zwei Anwendungsbedingungen der Gerechtigkeit unterschieden, nämlich einmal die (objektive) Bedingung der »gemäßigten Güterknappheit« sowie zweitens die (subjektive) Bedingung des »eingeschränkten Egoismus« (Schramme 2006, S. 30). Humes Idee war dabei die Feststellung, dass Fragen der Gerechtigkeit generell nur unter gewissen Bedingungen interessieren, nämlich wenn erstens weder Überfluss noch absolute Knappheit der Ressourcen herrschen, und zweitens, wenn die Subjekte nicht völlig altruistisch oder völlig egoistisch agieren. Denn wenn Überfluss herrscht und eine Ressource für alle in ausreichendem Maß vorhanden ist, so bedarf es keiner Überlegungen zur Verteilungsgerechtigkeit; dasselbe ist der Fall, wenn absolute Knappheit vorherrscht, so dass beim besten Willen nicht daran zu denken ist, eine gerechte Verteilung durchzuführen. Auch im Fall hochgradig selbstlos und altruistisch agierender Akteure (z.B. einer Republik von Heiligen) bedarf man keiner Überlegungen zur Verteilungsgerechtigkeit, und dasselbe Ergebnis tritt ein, wenn alle Akteure so 
egoistisch agieren, dass sie jeweils alle Ressourcen für ihre eigenen Zwecke einfordern. In diesem Zusammenhang ist es eine gute Frage, wie z.B. der Homo oeconomicus hinsichtlich der subjektiven Gerechtigkeitsbedingung agiert, und ob dieser als »eingeschränkter« oder als »absoluter« Egoist zu charakterisieren ist.

Wenn man so will, ist (gemäßigte) Güterknappheit also eine der Voraussetzungen und damit auch eines der konstitutiven Elemente jeder Debatte über Verteilungsgerechtigkeit. Diese gemäßigte Güterknappheit, so können wir mit Sicherheit feststellen, gehört nun auch zu den konstitutiven Elementen unseres Gesundheitssystems, so dass Überlegungen zur Gerechtigkeit (ganz im Einklang mit Humes Beobachtungen) hier am rechten Platz sind.

Von einer Mengenangabe als solcher können wir jedoch nicht auf die Knappheit einer bestimmten Ressource schließen. Knapp, ausreichend oder im Überfluss vorhanden ist eine Ressource nur in Hinsicht auf den Bedarf und die Ziele, die damit verfolgt werden sollen. Dementsprechend kann die Knappheit wiederum auf zwei konstitutive Elemente zurückgeführt werden, nämlich die Menge der Ressourcen und dem Bedarf danach. Entsprechend hat Schramme (Schramme 2006, S. 31f.) die oben angesprochene Objektivität des Knappheitskriteriums auch in Frage gestellt und unter Verweis auf den Bedarf von »relationaler Knappheit« gesprochen. Wir können folgern, dass mit Knappheit auf mindestens zwei verschiedene Arten umgegangen werden kann, nämlich einmal mit einer Erhöhung der Menge der zur Verfügung stehenden Ressourcen (wenn dies möglich ist), zum anderen mit einer Senkung des Bedarfs (was z.B. im medizinischen Bereich nicht so ohne Weiteres möglich ist). Es wird häufig als ein Axiom der Ökonomie dargestellt, dass die wirtschaftlichen Subjekte ein Interesse daran haben, von allen Ressourcen möglichst viel zu besitzen bzw. u.U. eine Monopolisierung der Ressourcen herbeizuführen. In seiner Arbeit Gerechtigkeit und soziale Praxis plädiert Schramme (Schramme 2006) aber mit überzeugenden Argumenten dafür, in der ethischen Diskussion an der Unterscheidung zwischen Bedürfnissen im engeren Sinne und darüber hinausführenden Wünschen nach einem Maximum an Res- 
sourcen zu unterscheiden. Nicht zuletzt führt eben das Streben nach einem »Mehr« an Ressourcen selbst wieder dazu, dass Knappheit entsteht: »Doch gerade die Auszeichnung des Wunsches nach so viel wie möglich führt erst zur relationalen Knappheit, denn nur wenn alle Ziele als legitim gelten, sind Güter notwendigerweise knapp« (Schramme 2006, S. 32). Zur Gerechtigkeitstheorie gehört also auch eine Debatte darüber, für welche Ziele Ressourcen im gesellschaftlichen Rahmen legitimerweise aufgewandt werden sollen.

\begin{tabular}{|l|l|}
\hline $\begin{array}{l}\text { Notwendig knappe Güter } \\
\text { Bsp.: Organe zur Transplantation }\end{array}$ & $\begin{array}{l}\text { Notwendig nicht knappe Güter } \\
\text { Bsp.: Luft, Wasser }\end{array}$ \\
\hline Kontingent knappe Güter & Kontingent nicht knappe Güter \\
Bsp.: Medizinische Versorgung & Bsp.: Lebensmittel, »ausreichend« \\
& vorh. Güter \\
\hline & \multicolumn{2}{|c|}{$\longrightarrow$} \\
\hline
\end{tabular}

Tabelle 2: Formen der Knappheit nach Schramme 2006, S. 33

Folgt man den Argumenten von Schramme, muss man vier verschiedene Arten von Knappheit bzw. der nicht vorhandenen Knappheit unterscheiden (vgl. Tabelle 2), die jeweils aus unterschiedlichen Gründen auftreten, damit aber auch unterschiedliche Implikationen für die Verteilungsgerechtigkeit haben: Notwendig knappe Güter sind deshalb knapp, weil sich ihre Menge nicht beliebig vermehren lässt. Dazu zählen z.B. die »positionellen Güter«, d.h. Güter, von denen es aus prinzipiellen Gründen immer nur sehr wenige gibt wie z.B. hohe Ämter im Staat. Im medizinischen Bereich ergibt sich die notwendige Knappheit teilweise aus anderen Umständen. So kann man zwar mit unterschiedlichen Gewinnungsregimes auf die Menge der für Transplantationen zur Verfügung stehenden Organe einwirken. Sie bleiben jedoch notwendigerweise knapp, weil ihre Gewinnung mit hohem Aufwand verbunden ist und der Bedarf die 
Zahl der zur Verfügung stehenden Organe gewöhnlich übersteigt. Weiterhin gibt es die notwendig nicht knappen Güter, die laut philosophischer Tradition »im Überfluss « vorhanden sind. Auch diese Kategorie ist mit einer gewissen Vorsicht zu betrachten, da, wie wir heute wissen, auch die natürlichen Ressourcen endlich sind, und bezogen auf die Zahl der zu versorgenden Menschen ernsthafte Limitierungen auftreten können.

In der nächsten Kategorie kommen nun mit dem Beispiel der medizinischen Versorgung die kontingent knappen Güter, die deshalb nur »kontingent knapp« sind, weil unsere Gesellschaft im Prinzip über genug Ressourcen verfügt, um solche Güter auch bedarfsdeckend oder sogar im Überfluss herzustellen. Aus Allokations- oder organisatorischen Gründen unterbleibt dies jedoch (es wird für wichtiger gehalten, die Ressourcen an anderer Stelle einzusetzen), so dass das Phänomen der Knappheit auftritt. Schramme führt an dieser Stelle auch das Beispiel der zur Verfügung stehenden Arbeitsplätze an. Es gab oder gibt Gesellschaftstypen, die in erster Linie versuchen, Arbeitsplätze zu schaffen und Fragen der Produktivität und Effizienz als weniger zentral ansehen. In Bezug auf die kontingente Knappheit ist nun die spannende Frage, ob ein rein marktwirtschaftliches System diese Form der Knappheit in der medizinischen Versorgung verschwinden lassen würde. Zu dieser Frage finden sich noch einige Überlegungen im weiteren Verlauf dieses Beitrages. Ein Beispiel, bei welchem der Zustand der Knappheit überwunden wurde, sind - zumindest in unserer Gesellschaft die kontingent nicht knappen Güter wie etwa Lebensmittel, aber auch die meisten Gebrauchsgegenstände des täglichen Lebens. Auch in der medizinischen Versorgung gibt es weite Bereiche der Versorgung kleinerer und größerer Erkrankungen (z.B. leichte Verletzungen, Brüche, Infektionen usw.), in denen de facto Versorgung ohne Knappheit stattfindet.

Umgangssprachlich gehen wir eher davon aus, dass es sich bei der Knappheit um einen problematischen Endzustand von Verteilungsprozessen handelt, bei welchem wir eben feststellen, dass eine Ressource nicht in ausreichendem Maß vorhanden und daher 
»knapp« ist. Folgt man der Hume'schen Analyse, so steht das Faktum der Knappheit (welches aus unterschiedlichen Faktoren resultiert) jedoch $z u$ Beginn der Diskussion um die gerechte Verteilung. Vergleichbar verhält sich die Sache interessanterweise in der klassisch-liberalen Nationalökonomie, die die Voraussetzung der Güterknappheit (oder anders gesagt: dass der Bedarf im Allgemeinen das Angebot übersteigt) als ein ökonomisches Grundaxiom oder die Voraussetzung allen Wirtschaftens betrachtet. So argumentiert Ludwig von Mises in seiner Nationalökonomie:

"Knappheit des Angebots im Hinblick auf den Umfang der Nachfrage ist Voraussetzung dafür, dass ein Gut überhaupt als wirtschaftliches Gut behandelt wird. Güter, die so reichlich zur Verfügung stehen, dass jede beabsichtigte Verwendung voll befriedigt werden kann, werden überhaupt nicht bewirtschaftet; für solche Güter werden keine Preise bezahlt" (von Mises 1940, S. 325).

Die Schlussfolgerung, welche die Ökonomie daraus zieht, ist jedoch eine andere als in der Theorie der Gerechtigkeit, nämlich dass knappe Ressourcen auf dem Markt gehandelt werden sollten. Dabei gehen die klassisch-marktwirtschaftlichen Ansätze mit einiger Emphase davon aus, dass der Markt, wenn er nur »richtig « reguliert und ausreichend liberalisiert ist, zugleich auch »gerecht« ist. Zusammenbrüche des Marktes werden daher gewöhnlich der Politik angelastet, die den Markt eben »falsch« reguliert hat, aber nicht der Gier der einzelnen Akteure. Gemeinhin negativ besetzte Phänomene wie Spekulation, exorbitante Preissteigerungen oder Insolvenzund Pleitewellen werden aus der Perspektive dieser Schulen als »Signale des Marktes« bzw. »schöpferische Zerstörung« (Schumpeter 2005) angesehen, aber zugleich als ethisch gerechtfertigt betrachtet, da die betreffenden wirtschaftlichen Akteure im freien Wettbewerb des Marktes versagt haben.

Dies führt notwendigerweise zu einem Konflikt zwischen ethischer und ökonomischer Theorie in Bezug auf das Phänomen der Knappheit, der zum jetzigen Zeitpunkt noch nicht als aufgelöst be- 
trachtet werden kann. Einerseits kannte auch schon Aristoteles die Tauschgerechtigkeit als eine »Gerechtigkeit des Marktes« und setzte voraus, dass ein freiwilliger Gütertausch nur zustande kommen kann, wenn beide Tauschparteien von der Gleichwertigkeit der auszutauschenden Güter ausgehen (Aristoteles Nikomachische Ethik 2008, Buch V). Aus dieser Voraussetzung kann jedoch auch ein Zustand der Güterknappheit resultieren, wenn der Tausch aufgrund von Armut oder ungleich verteilter Ressourcen nicht zustande kommen kann. Auf der anderen Seite hat sich die Ökonomie mit den Neuerungen des 18. Jahrhunderts, wie sie sehr deutlich bei Adam Smith in The Wealth of Nations ausgeführt werden, von der traditionellen antiken ebenso wie der christlichen Ethik entfernt. Bekanntlich setzten Smith und andere Autoren ja voraus, dass der Egoismus der Marktakteure ebenso das Gemeinwohl und den »Reichtum der Nationen« befördert. Die antike Ethik, insbesondere in der einflussreichen aristotelischen Ausprägung, setzte demgegenüber auf eine Ökonomie des Maßhaltens, eine Ethik der »Goldenen Mitte« und der Vermeidung der Extreme und kritisierte das »Mehr-Haben-Wollen« (als aristotelischer Fachbegriff die Pleonexie) als eine Form der Ungerechtigkeit.

\section{Ressourcenknappheit in der Medizin}

Verfolgen wir den Gedanken aus dem letzten Abschnitt weiter, kommen wir in Bezug auf das Problem der Ressourcenknappheit in der Medizin zu dem Ergebnis, dass exakt in diesem Bereich eine Parallelität von marktförmiger Verteilung und anderen Verteilungsformen besteht. Es wurde verschiedentlich diskutiert, inwiefern Ressourcen aus dem Bereich des Gesundheitswesens von einer ökonomischen und marktförmigen Verteilung ausgenommen werden sollten. Dazu werden im Folgenden in verkürzter Form einige 
Argumente angeführt, die in der bisherigen Diskussion eine Rolle gespielt haben ${ }^{11}$ :

- Insbesondere der schwerkranke Patient befindet sich nicht in einer Situation, in welcher er sich als Käufer oder Konsument frei und unabhängig für oder gegen den Erwerb einer bestimmten medizinischen Dienstleistung entscheiden kann.

- Die Beziehung zwischen Arzt und Patient ist keine rein geschäftliche, sondern der Patient erwartet vom Arzt einen Vorschlag, der im Sinne des besten Interesses des Patienten und seiner Gesundheit sein soll.

- Die Konsequenzen der marktförmigen Verteilung wie z.B. die Ungleichheit der Verteilung nach dem Vermögen der potenziellen Käufer, unerschwingliche Preise seltener und aufwendiger Behandlungen sowie die unterbleibende Befriedigung medizinischer Bedürfnisse sind im Gesundheitswesen unakzeptabel.

- Wenn der Staat die Verpflichtung hat, eine Basisversorgung mit existenziellen Gütern bereitzustellen, darf er gravierende Unterversorgung einzelner Individuen oder ganzer Bevölkerungsgruppen aufgrund gesellschaftlicher Ungleichheit nicht tolerieren.

Aus ökonomisch-marktliberaler Sicht wären alle diese Argumente zu kritisieren, da es ohne einen Markt mit freier Preisbildung auch nicht zu einer adäquaten Ressourcenallokation kommen kann. Insbesondere im medizinischen Bereich kommt aber dem in Tabelle 2 nach Schramme abgeleiteten Zusammenhang einige Bedeutung zu. Einige der Güter im medizinischen Bereich sind »notwendigerweise « knapp (wie z.B. Organe), da sie nicht beliebig vermehrbar sind bzw. da ihre Beschaffung (ob postmortal oder im Rahmen einer Lebendspende) die vorrangigen Rechte anderer Personen tangiert. Es gibt keine absolute Pflicht zur Organspende, und eben-

11 Siehe dazu z.B. die Beiträge in den Sammelbänden von Heubel et al. 2010 sowie Wild et al. 2011. 
so gibt es kein absolutes Recht, ein Organ zu erhalten, da dies die anerkannten Rechte anderer Personen berührt, die wiederum das Recht haben, über ihre Organe selbst entscheiden zu dürfen. Dieser Zusammenhang besteht unbenommen der Tatsache, dass es verschiedene Regelungen zur Organbeschaffung geben kann, die mehr oder weniger Organe zur Transplantation zur Verfügung stellen können.

Abhängig von der historischen und ökonomischen Entwicklung haben wir es in der Medizin überwiegend mit »kontingent knappen Gütern«, aber teilweise eben auch mit »kontingent nicht knappen Gütern« zu tun. Die kontingent knappen Güter sind deshalb knapp, weil sich die Gesellschaft (bzw. die dafür verantwortlichen Personen oder Institutionen) aufgrund expliziter oder impliziter Entscheidungen dazu entschlossen hat, nicht mehr von diesen Gütern produzieren zu lassen. Diese Entscheidung kann nun aus ethischer Sicht gut oder weniger gut begründet sein. Wie weiter unten noch zu klären sein wird, existieren aber jedenfalls Grenznutzenentscheidungen, bei denen die Kosten sehr hoch, aber der potenzielle Nutzen sehr gering ist, so dass sich eine entsprechende Behandlung mit Blick auf das Gesamtbudget verbietet. Die Behandlung könnte erbracht werden, aber die Kosten wären unverhältnismäßig im Vergleich zum Nutzen, so dass das bestehende Budget bei Erbringung derartiger Leistungen gesprengt werden würde und man dann Einsparungen in anderen gesellschaftlichen Bereichen vornehmen müsste (also in Bereichen wie Bildung, Arbeitsmarkt, Rüstung, Wirtschaftsförderung usw.). Es ist eine interessante Frage, ob diese Behandlungen unter einem Marktregime erbracht werden würden, d.h. wenn allein Angebot und Nachfrage die Preise und damit auch die in Anspruch genommenen Leistungen regulieren würden. Unter einem Marktregime wäre die Art der erbrachten Leistungen sowie die gesamte Versorgungssituation jedenfalls eine andere als in der Situation einer obligatorischen Versicherungspflicht.

Eine interessante Einsicht besteht jedenfalls darin, dass es auch im Bereich der Gesundheitsversorgung große Bereiche gibt, in denen 
wir letztlich kontingent nicht knappe Güter vorfinden. Viele Arten medizinischer Eingriffe (z.B. die Versorgung eines gebrochenen Beines) sind einerseits wichtig und andererseits mit den gegebenen Ressourcen unproblematisch $\mathrm{zu}$ erbringen. Mit ihrer Erbringung wird zugleich der bestehende Bedarf gedeckt.

Wie Franz Porzsolt (Porzsolt 2011, S. 8off.; vgl. Gossen 1854) ausführt, sind im Verhältnis von Ressourcenaufwand zu generierendem Nutzen die beiden Gossenschen Gesetze auch und gerade im Bereich der medizinischen Versorgung einschlägig. Das erste Gossensche Gesetz oder Gesetz vom abnehmenden Grenznutzen besagt, »dass der Konsum eines Gutes mit zunehmender Menge einen immer geringeren Zusatznutzen (Grenznutzen) stiftet« (Porzsolt, S. 8o) Wenn wir eine genügend leistungsfähige Infrastruktur für die Versorgung gebrochener Arme und Beine vorhalten, so stiftet es keinen zusätzlichen Nutzen, wenn wir noch weitere Ressourcen in diesen Bereich investieren. Das zweite Gossensche Gesetz bezieht sich auf die Frage, wie Ressourcen innerhalb eines Haushaltes oder eines Budgets derart auf verschiedene Bedürfnisse (oder z.B. medizinische Behandlungsformen) verteilt werden können, dass der größte mögliche Gesamtnutzen entsteht. Die ökonomisch optimale Lösung besteht darin, die Ressourcen so auf verschiedene Bedürfnisse (oder z.B. medizinische Behandlungsformen) zu verteilen, dass der »Grenznutzen für alle Güter, jeweils geteilt durch den Preis des Gutes, übereinstimmt« (Porzsolt, S. 80).

Eine Person, die mit einem Budget haushalten muss, wird deshalb ihre Ausgaben so auf die einzelnen Bereiche verteilen, dass erstens alle fundamentalen Bedürfnisse abgedeckt werden und zweitens alle weiteren Bedürfnisse, gemessen an ihrer Wichtigkeit, in einem ähnlichen Ausmaß befriedigt werden, so dass daraus dann ein vergleichbarer Nutzen entsteht. Wer Ressourcen über ein vertretbares Maß des Grenznutzens hinaus in eher unwichtigen Bereichen verschleudert, der läuft Gefahr, für die entscheidenden Lebensbereiche keine Mittel mehr übrig zu behalten. Ebenso sollten wir in der medizinischen Versorgung nur dann z.B. große Ressourcen in den Bereich der MRT-Diagnostik investieren, wenn 
wir damit einen vergleichbaren Nutzen wie in anderen Bereichen erzielen. Andernfalls sollten diese Mittel besser dort eingesetzt werden, wo ein besseres Kosten-Nutzen-Verhältnis besteht. Wenn die in die einzelnen Bereiche investierten Mittel einen vergleichbar großen therapeutischen Nutzen für die jeweilige Patientengruppe erzielen, dann wird auch der Gesamtnutzen für alle Patienten am Größten sein.

Vor dem Hintergrund dieser Überlegungen wird verständlich, dass in einigen Gesundheitssystemen wie z.B. dem britischen NHS eine universelle Kostengrenze (30.000 Pfund) pro gewonnenem QALY als Richtwert zur Beurteilung einer neuen therapeutischen Maßnahme angewandt wird. Denn ein solcher Richtwert verhindert zugleich, dass in einigen Bereichen des Gesundheitswesens übermäßig teure Mittel angewandt werden, ohne einen entsprechenden Nutzen zu generieren. Dieses utilitaristische Kalkül, das für den einzelnen Patienten durchaus inhumane Konsequenzen haben kann (und deshalb von vielen Kritikern kategorisch abgelehnt wird), hat in der Mittelverteilung aus der »Systemsicht« also durchaus eine gewisse Berechtigung. Zugleich wird plausibel, warum ein reines Marktsystem wie das U.S.-amerikanische in diesem Fall weniger effektiv ist als die gesteuerten Systeme, da es weder die Grenznutzenproblematik (das erste Gossensche Gesetz) noch die Verteilung entsprechend den verschiedenen Gesundheitsbedürfnissen einer Bevölkerung adäquat berücksichtigt.

Bereits im Abschnitt zu den allgemeinen Überlegungen zur Knappheit wurde festgestellt, dass wir von einer bestimmten Menge an Ressourcen oder einem bestimmten Anteil am Bruttosozialprodukt noch nicht auf den Grad der Knappheit einer Ressource schließen können. Ob eine bestimmte medizinische Versorgung zu knapp oder ausreichend vorhanden ist, ergibt sich erst in Hinblick auf den Bedarf und die Wichtigkeit der Ziele, die wir mit einer bestimmten Ressource verfolgen. Diese Wichtigkeit wird in einem reinen Marktsystem anhand der Preisbildung bestimmt. Wie wir gesehen haben, sprechen aber im Bereich des Gesundheitssystems wichtige Argumente gegen eine Anwendung des Marktprinzips im 
Gesundheitswesen. Die Wichtigkeit einer bestimmten Behandlung im Vergleich zu anderen muss daher in einem demokratischen Prozess unter Berücksichtigung medizinischer, ethischer, rechtlicher und politischer Faktoren gewonnen werden. Man sollte allerdings nicht zu pessimistisch sein und dem »common sense« bei der Beurteilung dieser Fragestellungen vollständig misstrauen. Wie wir im nächsten Abschnitt sehen werden, gibt es durchaus nachvollziehbare Überlegungen, warum bestimmte medizinische Behandlungen wichtiger sind als andere und daher auch prioritär erbracht werden sollten.

\section{Der Umgang mit begrenzten Ressourcen}

\subsection{Ergebnisse der norwegischen Kommission zur Priorisierung der Gesundheitsversorgung}

Insbesondere in den skandinavischen Ländern gibt es bereits seit den 1980ern Jahren eine dezidierte Diskussion zur Verteilung medizinischer Ressourcen. Diese Diskussion ist für uns an dieser Stelle gerade deshalb besonders interessant, weil auch dort die Entscheidung getroffen wurde, keine rein marktmäßige Steuerung des Gesundheitswesens vorzusehen, sondern sich vielmehr über sinnvolle medizinische Kriterien der Ressourcenverteilung Gedanken zu machen. Die Norwegische Kommission zur Priorisierung der Gesundheitsversorgung entwickelte aus dieser Diskussion heraus eine Stufenleiter der Priorität von Gesundheitsleistungen (Tabelle 3). 
Stufe 1

Lebensrettende Maßnahmen

Stufe 2

Notwendige Eingriffe mit guter Wirksamkeit, um die

langfristige Schädigung von Patienten zu vermeiden

Stufe 3

Eingriffe mit dokumentierter Wirkung, bei denen die

Folgen einer Nicht-Behandlung weniger schwer sind

Stufe 4

Dienste, die nachgefragt werden, aber mit geringem oder

keinem Schaden bei Nicht-Behandlung

Priorität 0

Dienste, die nachgefragt werden, die aber keinen nach-

gewiesenen Nutzen bringen

Tabelle 3: Ergebnisse der Norwegischen Kommission (Lønning Committee) zur Priorisierung der Gesundheitsversorgung. Aus:

Norwegische Kommission 1987, Calltorp et al. 2009, S. 217f.; Sabik

und Lie 2008, S. 3.

Das Ergebnis ist auch deshalb interessant, weil die klassischen nutzenorientierten Kandidaten für Prinzipien der Ressourcenverteilung wie z.B. QALYs, aber auch das Patientenalter (etwa für aufwendige und teure Behandlungsformen) dabei keine Rolle spielen. Eine unterschiedliche »Wertigkeit« der Behandlung individueller Patienten wird damit vermieden.

Im Zentrum dieser Überlegungen stehen vielmehr die Vermeidung schwerwiegender Schädigungen, die medizinische Notwendigkeit der Eingriffe, die Wirksamkeit einer therapeutischen Maßnahme sowie der damit potenziell zu erbringende Nutzen. Der Nutzen bzw. nicht vorhandene Nutzen der Behandlungen für verschiedene Patientengruppen muss dabei (ähnlich der bisherigen Vorgehensweise im System der deutschen GKV) im Expertenkonsens ermittelt werden (im Einklang mit klinischen Studien usw.) 
Damit sind auch gewisse Anknüpfungspunkte an die »mittleren Prinzipien« der Medizinethik gegeben (Prinzip der Schadensvermeidung, Benefizienzprinzip), ohne aber grundsätzliche Bedenken preiszugeben. Medizinisch notwendige Behandlungen sollen in jedem Fall erbracht werden. Das vorhandene Budget wird dabei prioritär für die als wichtig definierten medizinischen Leistungen aufgewandt, während die unteren Behandlungsstufen nur nachrangig aus öffentlichen Mitteln finanziert werden. Unter der Prämisse, dass es im medizinischen Bereich mit den lebensrettenden Maßnahmen eine Kategorie von Eingriffen gibt, die in jedem Fall erbracht werden müssen, werden Überlegungen der Kosteneffizienz gegenüber Fragen der Effektivität in den Hintergrund gestellt. Die dahinterstehende Rationale zielt mehr darauf, notwendige und bekanntermaßen wirksame Eingriffe durchzuführen, nicht notwendige und wenig wirksame Eingriffe aber zurückzustellen. Eine solche Herangehensweise kann Forderungen von verschiedener Seite erfüllen, da auch unter ethischen und medizinischen Gesichtspunkten unwirksame und überflüssige Eingriffe nicht erbracht werden sollten. Wenn eine Krankheit nach allgemeiner Einschätzung eine gewisse Schwere und Behandlungsbedürftigkeit aufweist, wirksame Behandlungsmethoden bestehen, und die Nichtbehandlung bei der betroffenen Person eine weitere Schädigung hervorruft, kann sie in die Stufen 1-3 des in Tabelle 3 beschriebenen Schemas verortet und entsprechend in den Katalog der zu vergütenden Gesundheitsleistungen aufgenommen werden. Ein weiterer Vorteil einer solchen kategorialen Einteilung besteht darin, dass unangemessen wirkende Abwägungsentscheidungen (z.B. welche Behandlung ist »wichtiger«: die Versorgung eines Armbruchs oder die Behandlung einer Lungenentzündung) nach Art von League-Tables o.ä. vermieden werden können. Dass Gesamtbudget müsste dann entsprechend so gestaltet werden, dass Leistungen der Prioritätsstufen 1-3 in jedem Fall erbracht werden können, während es dem politischen Ermessen obliegt, darüber hinausgehende Behandlungen in den Leistungskatalog mit aufzunehmen. 
Die Effektivität medizinischer Eingriffe lässt sich nicht nur für ein Land erheben, sondern auch international, so dass man hier eine konzertierte Aktion der OECD-Staaten durchführen könnte. In diesem Bereich scheint es allerdings trotz einer Reihe internationaler Organisationen in punkto gemeinsamer Aktivitäten noch einige Defizite zu geben, so dass man nicht von einer ausreichenden Abstimmung und Weiterentwicklung vergleichbarer Gesundheitssysteme zum Beispiel der OECD-Länder oder der Länder der Europäischen Union ausgehen kann.

\subsection{Unterschiedliche Priorisierungs- und Verteilungsformen im Vergleich}

Worin besteht nun der Unterschied der im letzten Abschnitt beschriebenen Form der Priorisierung medizinischer Behandlungen im Vergleich zu einer globalen Bewertung von Gesundheitsleistungen mit QALYs bzw. einer Verteilung von Gesundheitsdienstleistungen auf einem deregulierten Markt? Wahrscheinlich wird eine sachkundig durchgeführte QALY-Bewertung von medizinischen Eingriffen tatsächlich zu einer Erhöhung der Zielgröße, also eben der Quality Adjusted Life Years führen. Wie wir gesehen haben, liegen die Probleme aber in der Akzeptanz der QALYs als Zielgröße sowie den damit verbundenen problematischen normativen Implikationen. Derselbe, z.B. lebensrettende Eingriff, bei derselben Krankheit, aber bei Patienten unterschiedlichen Alters angewandt, kann eine stark abweichende Anzahl von QALYs zum Ergebnis haben. Ebenso profitiert eine Patientengruppe mit einem durchschnittlich höheren Alter in der Bewertung des QALY-Kalküls weniger stark von einer Behandlung als eine jüngere Patientengruppe. Dasselbe gilt im Prinzip für Patientengruppen, die stärker oder weniger stark von Komorbiditäten betroffen sind. Eine Evaluation mit QALYs wird also zu dem paradoxen Ergebnis führen, dass bei Patienten höheren Alters mit vergleichsweise mehr Komorbiditäten ein insgesamt geringerer Behandlungsnutzen entstehen wird als bei jüngeren und gesünderen Patienten. Wie wir im letzten Absatz gesehen haben, 
konzentriert sich der Vorschlag zur Priorisierung der norwegischen Kommission aber auf die Schwere einer Erkrankung sowie die Effektivität der Behandlung und sieht von den Eigenschaften der Patienten weitestgehend ab. ${ }^{12}$

Eine ausschließlich marktwirtschaftliche Zuteilung medizinischer Ressourcen würde wiederum dazu führen, dass sich finanziell schlechter gestellte Patienten in Bezug auf ihr Vermögen all$\mathrm{zu}$ teure Behandlungen nicht leisten können. Die Situation sieht leicht anders aus, wenn man sich ein privatwirtschaftliches Versicherungssystem als Vergleichsstandard nimmt. Wenn in einem solchen System aber nicht ein breiter Behandlungsumfang als obligatorischer Standard definiert wird, muss es unweigerlich zum Phänomen der Unterversicherung kommen, welches dann nur durch staatliches Eingreifen verhindert oder wenigstens gemildert werden kann. Wie bereits im Abschnitt zum gesellschaftlich agglomerierten Nutzen ausgeführt wurde, kann ein marktwirtschaftliches System im Bereich der Gesundheitsversorgung zur Fehlallokation von Ressourcen führen, da Bedarf und medizinische Versorgung durch die Ressourcenverteilung in einer Gesellschaft gewissermaßen »überformt « werden. Die Orientierung an der Krankheitsschwere und der Effektivität einer Behandlung kann dem jedoch entgegen steuern.

12 | Die Schwere einer Erkrankung ergibt sich dabei letztlich aus den gesundheitlichen Einschränkungen und Gefährdungen des Patienten in einem dreidimensionalen Krankheitsmodell (subjektive, objektive, relationale Dimension; vgl. Lenk 2002 sowie Lenk 2011), während die Effektivität einer Behandlung (bezogen auf die jeweilige Erkrankung) aus den Heilungsraten, der Morbidität und Lebensqualität, sowie der Mortalität einer Patientengruppe abgeleitet werden muss. Auch wenn es unterschiedliche Ansätze zur Feststellung der Behandlungseffektivität gibt, so wird genau dieses Ziel von klinischen Arzneimittelprüfungen, Behandlungsregistern sowie Studien zur Anwendungsbeobachtung verfolgt. 


\section{Schlussfolgerungen}

Der vorliegende Beitrag hat versucht, das Phänomen der Knappheit und damit verbunden die Frage des Nutzens verschiedener Formen der Güterverteilung nicht wie üblich aus der Sicht einer einzigen wissenschaftlichen Disziplin mit der dabei zwangsläufigen Verengung der Perspektive darzustellen, sondern mehrere Perspektiven und Traditionen zu Wort kommen zu lassen. Dies entspricht auf der theoretischen Ebene Ansätzen zur Gerechtigkeit wie z.B. dem Walzerschen, die von einem Prinzipienpluralismus und unterschiedlichen »Sphären« der Gerechtigkeit ausgehen. Dem Bereich der Gesundheitsversorgung kommt dabei, wie oben ausgeführt wurde, eine besondere Bedeutung zu. Insbesondere in unserer heutigen Gesellschaft kann Gesundheit als eine der in der öffentlichen Meinung anerkannten Grundlagen individuellen wie gesellschaftlichen Wohlergehens gelten. Wie wir gesehen haben, produziert diese allgemein anerkannte Wichtigkeit als Nachfrage dabei fast zwangsläufig Formen der Knappheit, die jedoch insbesondere im Bereich der Medizin differenziert betrachtet werden müssen. Es gibt unterschiedliche Formen medizinischen Bedarfs, von denen viele relativ unproblematisch gedeckt werden können.

Die Wahrnehmung einer zunehmenden Kommerzialisierung oder Ökonomisierung der Medizin hat dabei die Aufmerksamkeit für die Frage geschärft, inwiefern marktwirtschaftliche Elemente Eingang in die medizinische Versorgung finden sollten. Während aus Sicht der liberalen Marktwirtschaft eine freie Preisbildung die Voraussetzung der Güterallokation darstellt, überwiegen in den meisten Gesundheitssystemen Formen staatlich regulierter Gesundheitsversicherungen. Die Argumente gegen einen deregulierten Gesundheitsmarkt wurden entsprechend dargestellt.

Die Überlegungen zur Ressourcenverteilung im Gesundheitswesen müssen zwei unterschiedlichen Maßstäben entsprechen, nämlich einmal übergeordneten Kriterien wie z.B. der Menschenwürde, die in demokratischen Staaten letztlich durch die Verfassung vorgegeben werden, und andererseits der Eigenlogik der »Sphären«, in 
denen eine besondere Konstellation aus der gesellschaftlichen Bedeutung eines bestimmten Gutes, bestimmten Formen der Knappheit und vorhandenen Möglichkeiten der Verteilung vorliegt. Im Bereich der Gesundheitsversorgung spielt dabei insbesondere die »Bedarfsgerechtigkeit« oder das »Bedarfsprinzip« eine wichtige Rolle.

Nach wie vor wird immer noch diskutiert, ob nicht persönliche Eigenschaften der Patienten wie z.B. das Alter zur Güterallokation in der Medizin herangezogen werden sollen. Im vorliegenden Beitrag wurde dabei das Problem der Güterallokation nach solchen Kriterien zumindest im Zusammenhang mit QALYs angerissen. Das in Tabelle 3 dargestellte Priorisierungsschema folgt jedoch der ethischen Tradition (und auch der landläufigen moralischen Intuition) insofern, dass bei Verteilungsfragen bei Gütern von existenzieller Bedeutung wie in einigen Bereichen der Medizin eine Allokation ohne Ansehen der Person vorgenommen werden sollte.

Dabei stellt eine solche Form der Priorisierung nach Ansicht des Autors ein gelungenes Beispiel dar, wie aus verschiedenen wichtigen Kriterien die normative Eigenlogik eines Bereiches herausdestilliert werden kann. Auch die Auseinandersetzung über die medizinischen Fragen z.B. der Effektivität einer Behandlung birgt aber leider in Zeiten verschärften Lobbyismus reichlich Konfliktstoff. Doch mit der Schaffung von Institutionen wie z.B. NICE (National Institute for Health and Clinical Excellence) in Großbritannien oder dem IQWiG (Institut für Qualität und Wirtschaftlichkeit im Gesundheitswesen) in Deutschland wurden in diesem Zusammenhang sicherlich die richtigen Schritte unternommen. Noch defizitär ist möglicherweise der internationale Vergleich sowie die Vernetzung der Gesundheitspolitik, um aus Entwicklungen und Erkenntnissen vergleichbarer Länder noch besser als bisher lernen zu können. 


\section{LITERATUR}

Aristoteles (2008): Nikomachische Ethik (übersetzt und hg. von Ursula Wolf). Reinbek bei Hamburg: Rowohlt.

Birnbacher, D. (2012): Wie gerecht ist das deutsche System der Gesundheitsversorgung? In: Brudermüller, G. und Seelmann, K. (Hg.): Zweiklassenmedizin? Würzburg: Königshausen \& Neumann, 9-30.

Bundesärztekammer (BÄK) (2011): (Muster-)Berufsordnung für die in Deutschland tätigen Ärztinnen und Ärzte - MBO-Ä 1997 - in der Fassung der Beschlüsse des 114. Deutschen Ärztetages 2011 in Kiel.Zu finden unter:www.bundesaerztekammer.de/downloads/ MBO_08_20111.pdf, Zugriff: 17.11.2012.

Calltorp, J.; Larivaara, M.; Johnsen, J. (2009): Changing Perceptions of Equity and Fairness. In: Magnussen, J.; Vrangbaek, K.; Saltman, R.B. (Hg.) (2009): Nordic Health Care Systems. Recent Reforms and Current Policy Challenges. Maidenhead/Berkshire (England): Open University Press, 214-232.

Entwistle, V.A.; Watt, I.S.; Bradbury, R.; Pehl L.J. (1996): Media Coverage of the Child B Case. British Medical Journal 312: 1587-1591.

Forst, R. (1996): Kontexte der Gerechtigkeit - Politische Philosophie jenseits von Liberalismus und Kommunitarismus. Frankfurt a.M.: Suhrkamp Verlag, 1996.

Gosepath, S. (2004): Gleiche Gerechtigkeit. Grundlagen eines liberalen Egalitarismus. Frankfurt a.M.: Suhrkamp Verlag.

Gossen, H.H. (1854): Entwickelung der Gesetze des menschlichen Verkehrs, und der daraus fließenden Regeln für menschliches Handeln. Braunschweig: Friedrich Vieweg \& Sohn.

Heubel, F.; Kettner, M.; Manzeschke, A. (Hg.) (2010): Die Privatisierung von Krankenhäusern. Ethische Perspektiven. Wiesbaden: Verlag für Sozialwissenschaften.

Hume, D. (1777): An Enquiry Concerning the Principles of Morals. Oxford: Clarendon Press 3rd Ed. 1975. 
Lenk, C. (2002): Therapie und Enhancement. Ziele und Grenzen der modernen Medizin. Münsteraner Bioethische Schriften, Bd. 2, Münster: Lit Verlag.

Lenk, C. (2011): Enhancement vor dem Hintergrund verschiedener Konzepte von Gesundheit und Krankheit. In: Viehöver, W.; Wehling, P. (Hg.): Entgrenzung der Medizin. Von der Heilkunst zur Verbesserung des Menschen? Bielefeld: transcript Verlag, 67-87.

Magnussen, J.; Vrangbaek, K.; Saltman, R.B. (Hg.) (2009): Nordic Health Care Systems. Recent Reforms and Current Policy Challenges. Maidenhead/Berkshire (England): Open University Press.

Malycha, A. (2011): Der Ausbau des neuen Systems (1949 bis 1961). In: Bundeszentrale für Politische Bildung (Hg.): Geschichte der $D D R$. Informationen zur politischen Bildung Nr. 312, Bonn, 1936.

Markus, N. (2006): Die Zulässigkeit der Sectio auf Wunsch. Eine medizinische, ethische und rechtliche Betrachtung. Frankfurt a.M.: Peter Lang Verlag.

Miller, D. (1995): Complex Equality. In: Miller, D.; Walzer, M. (Hg.): Pluralism, Justice and Equality. Oxford: Oxford University Press, 197-225.

Mises, L. von (1940): Nationalökonomie. Genf: Editions Union Genf. Musschenga, B. (1997): The Relation Between Concepts of Qualityof-Life, Health and Happiness. Journal of Medicine and Philosophy 22(1): 11-28.

Norwegische Kommission zur Priorisierung der Gesundheitsversorgung (1987): Retningslinjer for prioritering innen helsevesenet (Richtlinien zur Priorisierung der Gesundheitsversorgung). Oslo: Norges offentlige utredninger (NOU).

OECD (2011): Health at a Glance: OECD Indicators, OECD Publishing. $\mathrm{Zu}$ finden unter: http://dx.doi.org/10.1787/health_glance-2011en. Zugriff: 17.11.2012.

Porzsolt, F. (2011): Grundlagen der Klinischen Ökonomik. Berlin: PVS Verlag.

Rawls, J. (1999): A Theory of Justice. Revidierte Fassung, Cambridge, Mass.: Harvard University Press. 
Rüegger, H.; Pfister Lipp, E.; Heuss, L.; Hillewerth, K.; Widmer, W. (2012): Zur sogenannten Ökonomisierung des Gesundheitswesens. Ethik Med 24: 153-157.

Sabik, M.L.; Lie, R.K. (2008): Priority Setting in Health Care: Lessons From The Experiences of Eight Countries. International Journal for Equity in Health 7: 4.

Schramme, T. (2006): Gerechtigkeit und soziale Praxis. Frankfurt a.M.: Campus Verlag.

Schumpeter, J.A. (2005): Kapitalismus, Sozialismus und Demokratie. Stuttgart: UTB.

Walzer, M. (2006): Sphären der Gerechtigkeit. Ein Plädoyer für Pluralität und Gleichheit. Frankfurt a.M.: Campus Verlag.

Wild, V.; Pfister, E.; Biller-Andorno, N. (Hg.) (2011): DRG und Ethik. Ethische Auswirkungen von ökonomischen Steuerungselementen im Gesundheitswesen. Basel: EMH Schweizerischer Ärzteverlag. 



\section{Prinzipien einer gerechten Rationierung im Gesundheitswesen}

Walter Pfannkuche

\section{Was sind die Probleme?}

In der Diskussion über die Ausgestaltung des Gesundheitswesens stehen angesichts knapper Ressourcen heute auf verschiedenen Ebenen folgende Fragen im Mittelpunkt:

a) Auf der politischen Ebene wird diskutiert, wie groß das Globalbudget für medizinische Leistungen sein soll. Dabei muss von den Gesetzgebern das Gut der medizinischen Hilfe gegen andere Güter abgewogen werden - gegen Sicherheit, Bildung, Kultur usw. Hier setzt sich die Erkenntnis durch, dass die heutigen Finanzierungsprobleme im Gesundheitswesen durch eine Rationalisierung der entsprechenden Einrichtungen (Vermeidung von Doppeluntersuchtungen, Verkürzung der Verweildauer usw.) nicht zu lösen sind. Es gibt keine prästabilierte Harmonie der Art, dass nach Beseitigung aller Ineffizienzen gerade genug Geld für alle medizinisch sinnvollen Maßnahmen vorhanden ist. Unabhängig von der Vermeidung von Ineffizienzen stellt sich die Frage, wieviel Geld man überhaupt für Gesundheitsfürsorge ausgeben will.

b) Innerhalb des medizinischen Versorgungssystems geht es um die Frage, wie das medizinische Globalbudget verteilt werden soll. Für welche Typen von Maßnahmen (Prävention, Notfallversorgung, Intensivmedizin usw.) sollen wieviel Prozent der Mittel eingesetzt werden? Welche neuen Behandlungsmethoden sollen in den Leistungskatalog der Krankenkassen aufgenommen, welche 
sollen gestrichen werden? Eine wichtige Frage im Vorfeld solcher Abwägungen ist dabei die, woran die Erfolge der zu vergleichenden Maßnahmen gemessen werden sollen (QALY1 usw.).

c) Für den einzelnen Arzt oder den Leiter einer Krankenhausabteilung schließlich stellt sich die Frage, wie - im Rahmen der Vorgaben unter (a) und (b) - die Leistungen auf die Patienten verteilt werden sollen. Welcher Patient soll angesichts eines vorab definierten ökonomischen Rahmens welche Leistungen erhalten?

Die Überlegungen auf allen diesen drei Ebenen entscheiden über das Wohl und Wehe und zuletzt über das Leben und Sterben von Menschen. Diese Überlegungen werden gleichwohl kaum öffentlich kommuniziert und werden auch von Patienten normalerweise gar nicht oder als nicht beeinflussbare Vorgänge wahrgenommen. Der Patient sieht nur das Resultat: Bestimmte Leistungen werden von seiner Krankenkasse nicht oder nur teilweise bezahlt oder er erhält sie nur nach einer mehr oder weniger langen Wartezeit. Nur selten haben Patienten den Eindruck, dass ihnen essentielle Leistungen vorenthalten werden - etwa ein vielleicht wirksameres, aber auch erheblich teureres Medikament. Das liegt vor allem daran, dass solche Wahrnehmungen einen gut informierten Patienten voraussetzen. Zumeist kann der Arzt solche von Kostengesichtspunkten mitbestimmten Überlegungen vor dem Patienten verbergen. Er schlägt ihm eine Behandlungsmethode oder ein Medikament als die am besten geeignete Maßnahme vor, und das wird von den Patienten in den allermeisten Fällen schlicht akzeptiert.

Gleichwohl, es gibt solche Erfahrungen der Leistungsverweigerung und es gibt das allgemeine Gefühl, dass Privatversicherte eine bessere Gesundheitsfürsorge erhalten als Kassenpatienten, dass also etwas dran ist an dem alten Slogan »Wer arm ist, muß früher sterben«. Das vorzeitige Sterbenmüssen aus diesem Grund wird dabei als ein gravierendes Unrecht wahrgenommen. Wenn die Mitglieder der Gesellschaft auch sonst in vielen Hinsichten ver-

1 | Für eine Darstellung des QALY-Konzepts vgl. den Beitrag von Kathrin Dengler in diesem Band. 
schieden gut dastehen mögen, hinsichtlich der Gesundheit, so die vorherrschende Meinung, sollten doch alle gleich gut gestellt werden. Gesundheit, so meinen die meisten, ist ein so wichtiges Gut, dass auf deren Erhaltung und Wiederherstellung jeder Bürger ein gleiches Recht hat. Ungleichheiten an dieser Stelle werden als moralisches Versagen eines Gemeinwesens wahrgenommen.

\section{Das Modell der unparteitschen Abwägung}

Im Folgenden werde ich der Frage nachgehen, inwiefern die Vorenthaltung möglicher medizinisch sinnvoller Leistungen und inwiefern Ungleichheiten in der medizinischen Versorgung Formen von Unrecht darstellen. ${ }^{2}$ Das lässt sich nicht beantworten, ohne sich mit der allgemeineren Frage auseinanderzusetzen, wie wir überhaupt zu der Überzeugung kommen, Rechte zu haben. Wie werden Rechte konstituiert, also gerechtfertigt und etabliert?

An dieser Stelle sehen wir uns mit einer Vielzahl von Theorien konfrontiert, die einander mehr oder weniger stark widersprechen. Manche gehen von anspruchsvollen Voraussetzungen aus. Etwa davon, dass Rechte etwas Gott- oder Naturgegebenes sind, etwas, das in der menschengemachten Rechtsordnung lediglich abzubilden ist. Oder davon, dass jeder Mensch eine Würde besitzt, und dass diese als solche jedem anderen Menschen einen Grund gibt, ihn rücksichtsvoll zu behandeln. So kann man denken, aber solche Voraussetzungen sind erstens sehr umstritten und zudem ist zweitens dann strittig, was hinsichtlich der Gesundheitsfürsorge nun genau dem Willen Gottes oder der Würde des Menschen entspricht. Zur Herstellung eines möglichst breiten Konsensus erscheint es mir angeraten, nicht mit derartig großformatigen Begriffen zu arbeiten. Erfolgversprechender erscheint mir die Suche nach einer mi-

2| Für den im Folgenden entwickelten Lösungsvorschlag bin ich Ulla Wessels (Universität Saarbrücken) zu Dank verpflichtet. Ohne ihre Kritik und Anregungen wären meine Überlegungen schwerlich so weit gediehen. 
nimalistischen Begründungsstrategie. Das wäre eine, die nur mit solchen Elementen zu arbeiten versucht, die sich aus dem ergeben, was mit dem Einnehmen einer moralischen Perspektive überhaupt zusammenhängt. Wenn man überhaupt moralisch argumentieren will, was muss man dann beachten? Was charakterisiert eine moralische Denkweise, Einstellung oder Begründung?

Meine These hierzu lautet, dass die moralische Perspektive auf ein Problem durch die Frage zu charakterisieren ist: Welche Regelung oder Handlungsweise aller würde man in Hinblick auf ein Problem befürworten, wenn man unparteiisch darüber nachdenkt, d.h. wenn man sich vorstellt, dass man in jeder Rolle sein könnte, die zu dem Problem gehört?

Das Verfahren der unparteiischen Abwägung erfordert, sich in die Position aller Handlungsbetroffenen hineinzuversetzen und deren Interessen gleich wichtig zu nehmen. Zum moralischen Denken gehört es deshalb, die Vor- und Nachteile möglicher Handlungsweisen unter Berücksichtung aller Betroffenen neutral abzuwägen. Diese Aufforderung, die Sache einmal aus der Position des anderen $\mathrm{zu}$ betrachten, ist in der Tat die wohl weitestverbreitete Grundregel für moralisches Denken in der Geschichte der Menschheit. Wir finden sie als Goldene Regel ebenso in den Reden des Buddha wie in der Bergpredigt Christi (vgl. Lukas-Evangelium, VI, 31; Reden des Buddha 1993, S. 4off). Als moralisch richtig wird man jene Handlungsweise anerkennen, die sich nach unparteiischer Abwägung aller damit verbundenen Vor- und Nachteile als die am meisten $\mathrm{Zu}$ friedenstellende erwiesen hat.

Auf diese Weise kann man den Begriff des Rechts so definieren: Rechte werden dann all diejenigen Handlungsweisen oder Prinzipien sein, von denen man aus der unparteiischen Perspektive wollen würde, dass sie durch ein für alle gültiges Regelsystem etabliert und durch ein allgemeines Sanktionssystem gestützt werden. ${ }^{3}$ Zwei Beispiele zur Verdeutlichung:

3 I Rechte kann man nur auf etwas haben, was man von anderen als Handlung oder Unterlassung erzwingen kann. Es kann ein Recht auf elterliche Fürsorge geben, aber keines auf Liebe. 
- Wenn mein Leben gegen Angriffe anderer durch ein Recht geschützt wird, dann kann ich ruhiger leben, aber dann muss ich meine Neigung, mir unliebsame Mitmenschen aus dem Weg zu räumen, ebenfalls zügeln, weil ich sonst mit rechtlichen Sanktionen $\mathrm{zu}$ rechnen habe.

- Wenn ich ein Recht auf Bildung haben will, dann muss ich auch bereit sein, zu den dafür notwendigen Einrichtungen beizutragen.

Auf der Basis dieses Modells ist man mit zwei Problemen konfrontiert, die sich daraus ergeben, dass Menschen sowohl in ihrer Gewichtung verschiedener Güter, wie auch in ihrer Risikobereitschaft voneinander abweichen:

Erstens: Diese Unterschiede werden auf der allgemeinsten und grundlegenden Ebene zur Befürwortung unterschiedlicher Aggregationsprinzipien führen. Zum moralischen Denken gehört das Gleichwichtignehmen der Interessen aller Betroffenen. Aber was soll man tun, wenn man im Zuge dieser unparteiischen Betrachtung bemerkt, dass die Interessen der Subjekte einander auf vielfache Weise entgegengesetzt sind und infolge knapper Ressourcen nicht alle vollständigt befriedigt werden können? Was soll man tun, wenn man, was immer man tut, nicht alle vollständig glücklich machen kann? Soll man dann - wie es die Utilitaristen ${ }^{4}$ befürworten - auf die Maximierung des Gesamtnutzens zielen? Oder - wie die Egalitaristen - eher auf die Gleichverteilung des Wohlbefindens? Oder ist beides falsch und es geht - wie die Rawlsianer meinen darum, die schlechtestmögliche Position so gut wie möglich auszugestalten (Maximin-Prinzip)?

Zweitens: Auch innerhalb einer jeden dieser Fundamentaltheorien gehen die Meinungen darüber auseinander, wodurch das Wohl einer Person gemehrt wird. Personen haben normalerweise eine Vielzahl von Interessen, von deren Befriedigung ihr Wohlbe-

4 | Für eine ausführliche Darstellung des Utilitarismus siehe auch den Beitrag von Günter Fröhlich in diesem Band. 
finden abhängt. Wer moralisch über ein Problem nachdenken will, müsste also wissen, wie wichtig welche dieser Interessen für eine Person sind, und er müsste in der Lage sein, die Zufriedenheitszustände von Personen miteinander zu vergleichen. Das scheint ein hoffnungslos überkomplexes Unterfangen zu sein. Angesichts dieses Ozeans der Interessen besteht eine plausible Entlastungsstrategie vieler Moraltheorien darin, sich auf grundlegende, so genannte transzendentale Interessen zu beschränken. Darunter versteht man das Interesse an Gegebenheiten oder Dingen, über die eine Person notwendig verfügen muss, wenn sie überhaupt irgendwelche Ziele verfolgen will. So muss man gesund sein und über bestimmte Kenntnisse und einen geschützten Freiheitsspielraum verfügen, um seine spezifischen Ziele wirksam verfolgen zu können. Die Konzentration auf diese transzendentalen Interessen entlastet das moralische Denken von vielen Problemen, aber sie kann das Gewichtungsproblem nicht umschiffen, denn auch innerhalb der transzendentalen Güter stellen sich Gewichtungsfragen. Geht es einer Person besser, wenn sie über einen großen Freiheitsspielraum und wenig Absicherung verfügt, oder geht es ihr besser, wenn diese Güter andersherum gewichtet sind?

Die hier möglichen und wahrscheinlichen Divergenzen werden sich insbesondere bei der Bestimmung des Kanons der akzeptablen positiven Rechte auswirken. Ihrer Struktur nach sind positive Rechte Ansprüche auf bestimmte Leistungen und damit zumeist Absicherungen gegen typische Lebensrisiken. Betrachten wir gleich das positive Recht auf medizinische Fürsorge: $\mathrm{Ob}$ man Krebs bekommt oder nicht, ist (artifiziell erzeugte Belastungen mal außen vor gelassen) eine Frage von Glück oder Pech. Aber in modernen Gesellschaften stehen die Bürger vor der Wahl, ob sie im Erkrankungsfall dem natürlichen Lauf der Dinge ausgeliefert bleiben oder ob sie ein zusätzliches Sicherheitsnetz in Form eines Anspruchs auf medizinische Betreuung einziehen wollen. Da eine Krankenversicherung Geld kostet, muss man nun abwägen, wieviel mehr an Sicherheit einem wieviel wert ist, d.h. welche Einschränkungen in anderen Lebensbereichen man dafür hinzunehmen gewillt ist. 


\section{WAS FOLGT DARAUS FÜR EIN RECHTFERTIGBARES GESUNDHEITSWESEN?}

Jeder der oben angeführten Fundamentaltheorien wird ein anderer Begriff der Gerechtigkeit entsprechen. Wenn die Anhänger des Maximin-Prinzips es für geboten halten, Ressourcen speziell zur Verbesserung der schlechtestmöglichen Position einzusetzen, werden Utilitaristen dies als eine Vergeudung von Ressourcen begreifen, wenn sich mit einer anderen Verwendung der Mittel das Gesamtglück besser vermehren lässt. Zwischen diesen Fundamentaltheorien und den ihnen entsprechenden Gerechtigkeitsbegriffen gibt es einen ewigen Streit. Es scheint mir deshalb wenig erfolgversprechend, einen weiteren Beitrag dazu zu leisten. Angemessener und fruchtbarer erscheint es mir, diese Fundamentaltheorien als Positionen eines vertretbaren moralischen Pluralismus zu begreifen und im Folgenden zu erkunden, was man angesichts dieses Pluralismus hinsichtlich einer für alle akzeptablen Rechtsordnung noch sagen kann. Wie kann man angesichts des moralischen Pluralismus und angesichts der abweichenden Güterabwägungen - insbesondere hinsichtlich des Gutes Gesundheit - noch zu Rechten kommen, die alle für gerechtfertigt halten können?

Man könnte vermuten, dass sich hinsichtlich des Gutes einer Krankenversicherung hier kein großes Problem ergibt. Denn naheliegende Kandidaten für die Absicherung durch rechtsförmigen Schutz sind ja all die Güter, die uns besonders wichtig sind. Die Gesundheit gehört klarerweise dazu und ist also prima facie innerhalb jeder der Theorien im Rahmen des moralischen Pluralismus ein plausibler Kandidat für eine rechtsförmige Absicherung. Doch diese anfängliche Gemeinsamkeit trägt nicht sehr weit: Zwar ist ein rechtsförmiger Schutz des Gutes Gesundheit insofern unstrittig, als es um das Verbot von Verletzungshandlungen geht. Jeder fürchtet sich vor den Beschädigungen, die andere ihm zufügen können. Und nahezu jeder wird auch angesichts der Einschränkungen, die ihm das selbst auferlegt, die Sanktionierung von Verletzungshandlungen, befürworten, eben deshalb, weil die Zügelung der eigenen 
Aggressionen weniger übel ist als das Verletztwerden oder die ständige Furcht davor. Weitaus weniger einhellig fallen jedoch die Urteile hinsichtlich des Umfangs der positiven Absicherungsintensität aus. Zwar werden die meisten die Einrichtung eines Polizeiwesens zum Schutz vor Beschädigungen durch andere befürworten. Aber schon bei der Frage, wieviele Mittel für diesen Schutz ausgegeben werden sollen, wie gut der Schutz also sein soll, werden die Meinungen auseinandergehen. Und diese Divergenzen werden noch größer sein, wenn es um die Höhe der Absicherungsintensität gegen Krankheiten geht.

Bei solchen Fragen geht es um Güterabwägungen - Gesundheit versus Kultur versus Bildung usw. Bei dieser Abwägung hilft es nichts, darauf hinzuweisen, dass Gesundheit ein transzendentales, alle anderen Güter erst ermöglichendes Gut ist. Kaum jemand wird alles Mögliche für seine Gesunderhaltung tun wollen, bevor er sich eines der anderen guten Dinge im Leben gönnt (etwa eine Urlaubsreise). Auch wenn man ggf. alle seine Ressourcen aufwenden würde, um eine eingetretene Krebserkrankung zu behandeln, daraus folgt nicht, dass man präventiv alle verfügbaren Ressourcen beiseite legen würde, um im Fall des Eintretens möglichst gut gewappnet zu sein. Diese Einschränkung gilt auch für das weite Feld präventivmedizinischer Maßnahmen wie Luftreinhaltung oder Vorsorgeuntersuchungen. Daraus, dass die Gesundheit ein transzendentales Gut ist, folgt nicht, dass ein rationaler Mensch alle verfügbaren Ressourcen für die Minimierung des Eintretensrisikos von Erkrankungen aufwenden würde. Es gibt hier keine in der Natur der rationalen Abwägung begründete absolute Priorität. Jeder muss für sich abwägen, und die Individuen werden zu verschiedenen Resultaten kommen. Deshalb ist es auch irreführend, angesichts schwer erkrankter Menschen darauf hinzuweisen, diese hätten doch einen Anspruch auf Hilfe, das ergebe sich unmittelbar aus ihrer Würde als Menschen. Natürlich werden diese Menschen sich dringend wünschen, dass ihnen geholfen wird. Aber daraus folgt eben noch nicht, dass 
sie in unparteiischer Abwägung auch selbst bereit sind, die Mittel aufzubringen, die solche Hilfe ermöglichen würden. ${ }^{5}$

Es ist also keineswegs so, dass ein rechtsförmiger Anspruch auf Gesundheitsfürsorge zum unstittigen Kern aller plausiblen Moraloder Rechtstheorien gehört. Bei jeder in einem Gemeinwesen für alle rechtlich verbindlichen Regelung wird es, eben weil die Güterabwägungen der Individuen voneinander abweichen, vielmehr Personen geben, die die realisierte Absicherung zu stark oder zu schwach finden. Diese Divergenzen kann man nicht rational auflösen, man kann hier nur eine zweite Reflexionsebene betreten und fragen: Wie soll ein Gemeinwesen mit solchen Divergenzen umgehen?

\subsection{Individualisierung}

Angsichts dieser abweichenden Beurteilungen besteht ein möglicher Ausweg darin, in dem betreffenden Lebensbereich auf eine für alle rechtsverbindliche Regelung zu verzichten. Man etabliert keine staatlich garantierten Anspruchsrechte. Jeder muss die für ihn angemessene, seinen Präferenzen entsprechende Absicherungsintensität wählen. Eine Krankenversicherung wäre strukturell dann dasselbe wie eine Hausratversicherung. Deren Vorhandensein und Beschaffenheit wäre gar kein moralisch bedeutsamer Tatbestand mehr.

5 | Eben deshalb ist es auch vorschnell, aus Art.2, Abs.2 des GG ("Jeder hat das Recht auf Leben und körperliche Unversehrtheit") abzuleiten, dass dem Recht auf Leben positive Rechte korrespondieren und dass es einen Kernbereich der Leistungspflicht gibt, zu dem in jedem Fall alle Leistungen gehören, die möglich und erforderlich sind, um lebensbedrohliche Krankheiten zu bekämpfen. Der Artikel-Text ist vielmehr so allgemein, dass nicht einmal klar ist, ob damit überhaupt positive oder nur Unterlassungspflichten gemeint sind. Dementsprechend könnte die staatliche Schutzpflicht sich auch darin erschöpfen, aktive Angriffe auf Leben und Gesundheit zu unterbinden oder zu sanktionieren. 
Eine solche Individualisierung ist allerdings nur dann moralisch akzeptabel, wenn die Rahmenbedingungen der individuellen Wahlentscheidungen moralisch akzeptabel sind. Das erfordert mindestens, dass die für die Wählenden verfügbaren Ressourcen (Einkommen, Vermögen usw.) gerecht verteilt sind. Auf der Basis des moralischen Pluralismus kann dies nun nicht mehr bedeuten, dass die Verteilung dem einen einzig wahren Verteilungsprinzip genügt. Unter den Bedingungen des moralischen Pluralismus kann die für eine Gesellschaft gültige Gerechtigkeitsnorm nur diejenige unter den plausiblen Gerechtigkeitsnormen sein, die die Mitglieder der Gesellschaft in einem Akt politischer Selbstbestimmung als für sich gültig angenommen haben. Ausgehend von einer so etablierten und realisierten Gerechtigkeitskonzeption ist eine individuelle Wahl der Absicherungsintensität aus der unparteiischen Perspektive vertretbar, weil diese Wahl eine wichtige Form der Selbstbestimmung ist und weil Selbstbestimmung sicher ein zentrales Gut in einer jeden Konzeption des guten Lebens ist - und damit ein wichtiges Element in einer jeden Moral, die das Wohl der moralischen Subjekte ernst nehmen will. ${ }^{6}$

Bei gerechten Ausgangsbedingungen könnte sich niemand mehr beklagen, dass er früher sterben muss, weil er sich keine bessere Versicherung leisten konnte. Solange die Ressourcen gerecht verteilt sind, mag es bedauerlich sein, dass die Ressourcen der Individuen für ein besseres Gesundheitssystem nicht ausreichen, aber dessen Fehlen ist dann kein Fall von Ungerechtigkeit, sondern einfach Ausdruck des Entwicklungsstandes einer Gesellschaft.

Bei dieser Individualisierung hinsichtlich des Umfangs der gewählten Gesundheitsfürsorge liegt eine zweiteilige Struktur vor:

Zum einen wird auf die Frage der Einkommens- oder Ressourcenverteilung direkt das Gerechtigkeitsprinzip angewandt, für das

6 | Sicher können die Individuen sich in ihren Güterabwägungen irren, aber eine staatliche Agentur zur flächendeckenden Korrektur solcher Fehler wäre vermutlich ein noch größeres Übel als die vorkommenden Fehler der Individuen. 
sich eine Gesellschaft in kollektiver politischer Selbstbestimmung entschieden hat. Und zum anderen kommen hinsichtlich der Frage der medizinischen Absicherungsintensität individuelle Präferenzen zur Geltung.

Diese Zweiteilung ist aus zwei Gründen plausibel:

Erstens: Von der Universalressource Einkommen will jeder lieber mehr als weniger haben. Hier kann man gleichgerichtete Interessen bei allen unterstellen. Und das ist bei dem Gut der Absicherung eben nicht der Fall.

Zweitens: Jede Gesellschaft braucht ein Gerechtigkeitsprinzip, nach dem sie organisiert ist. Man kann nicht zugleich in demselben Bereich nach zwei verschiedenen Gerechtigkeitsprinzipien verfahren. Man kann aber sehr wohl verschiedene Absicherungsniveaus in demselben Bereich haben, ohne dass das zu Widersprüchen führt. Hier ist Pluralismus möglich. Man brauchte schon starke Gründe, ihn nicht zuzulassen.

So charmant das Modell der individuellen Wahl einer Absicherungsintensität ist, es ist mit einer Reihe von Problemen konfrontiert:

1. Heranwachsende oder dauerhaft Hilflose oder Einkommenslose sind von der Entscheidung ihrer Eltern oder von staatlichen Institutionen abhängig. Welche Versicherungsintensität dürfen oder müssen diese für ihre Schutzbefohlenen wählen?

2. Die Wahl der Absicherungsintensität kann nur dann fair sein, wenn die Ausgangsbedingungen gerecht sind. Zwischen den Individuen gibt es aber unverschuldete genetische gesundheitsrelevante Unterschiede. Auch mit diesen muss aus der unparteiischen Perspektive umgegangen werden. D.h. die Wählenden können nicht einfach von ihrer faktischen genetischen Ausstattung ausgehen, sondern müssen sich auch hier vorstellen, in jeder möglichen Lage zu sein, und dann nach einer akzeptablen Lösung suchen.

3. Gesundheit ist partiell ein kollektives Gut. Wenn viele sich aus Kostengründen etwa gegen Schutzimpfungen oder andere Maß- 
nahmen zur Bekämpfung infektiöser Krankheiten entscheiden, dann erhöht das die Risiken auch derer, die solche Maßnahmen befürworten und ggf. auch individuell realisieren. Und umgekehrt würden Nicht-Impfer als Trittbrettfahrer von einer Majorität von Impfern profitieren.

4. Die Auswirkungen des Systems der Gesundheitsfürsorge auf andere Lebensbereiche müssen berücksichtigt werden. So könnten Un- oder Unterversicherte im Notfall zu kriminellem Verhalten neigen (Beschaffungskriminalität). Sie haben dann >nichts mehr $\mathrm{zu}$ verlieren $<$ und werden zur Gefahr für andere.

Diese Probleme rechtfertigen und erfordern auch bei gerechter Ressourcenverteilung einen gewissen staatlich bestimmten Ordnungsrahmen im Gesundheitswesen.

Ich werde auf diese Probleme hier nicht weiter eingehen. Sie sind zwar ernsthaft, aber eben nur die Probleme einer moralischrechtlichen Ideallösung. Von dieser ist jedoch unsere reale gesellschaftliche Situation in einer Weise verschieden, die andere und dringendere Probleme heraufbeschwört: In unserer Gesellschaft ist die Ausgangsposition der Individuen durch ungerechtfertigte ökonomische Ungleichheiten gekennzeichnet. Kaum jemand hält die Ressourcenverteilung in Deutschland für gerecht. Die Einkommenspreizung bei abhängig Beschäftigten etwa hat eine Größenordnung von 1:66o. Wenn die große Mehrheit der Bürger die Vermögensverteilung aber als ungerecht beurteilt, dann sind individuelle Entscheidungen über die gewünschte Absicherungsintensität moralisch defizitär, weil die Ausgangsbasis der individuellen Abwägungen auf ungerechtfertigte Weise ungleich ist. Hier würde bei einer von den individuellen Ressourcen bestimmten Wahl der Versicherungsintensität der Fall eintreten, dass Arme ggf. auch wegen ihrer Armut früher sterben müssen. Es stand ihnen eben nicht frei, eine bessere Absicherungsintensität zu wählen.

Wenn man das für einen moralisch anstößigen Sachverhalt hält, dann ist die für unsere Gesellschaft dringendste Frage: Durch welche Regelungen könnte trotz dieser Anfangsungerechtigkeit we- 
nigstens hinsichtlich der medizinischen Versorgung ein moralisch akzeptables Resultat erreicht werden? Die folgenden Strategien erscheinen erwägenswert:

\subsection{Second-best-Strategien}

Im Folgenden möchte ich nun drei Ansätze diskutieren, die Lösungsversuche innerhalb der genannten Rahmenungerechtigkeit darstellen und insofern als mögliche Second-best-Strategien zu verstehen sind.

1. Die Absicherungsintensität bzw. das Gesamtbudget für medizinische Leistungen wird politisch und für alle bindend rechtsförmig bestimmt. Personen, die eine stärkere Absicherung vorziehen und die Ressourcen $z u$ deren Finanzierung haben, dürften sich dann nicht individuell besser absichern.

Das würde zwar in Hinsicht auf Krankheit und Tod alle gleichstellen, es würde aber zugleich die Individuen in ihrer Selbstbestimmung stark beschneiden. Sie dürfen sich nicht besser absichern als es kollektiv bestimmt wurde. Eine so gravierende Beschneidung der Selbstbestimmung ist aber allenfalls dann vertretbar, wenn sich keine weniger dirigistischen Modelle entwickeln lassen. Ein solcher Dirigismus würde unter heutigen Bedingungen zudem zu einem Krankentourismus der Bessergestellten ins Ausland führen. D.h. der beabsichtigte Effekt, die Herstellung einer Gleichheit vor Krankheit und Tod, ist auf diese Weise nicht erreichbar.

Und umgekehrt würden auch diejenigen, die eine schwächere Absicherung als die gesetzlich vorgeschriebene vorziehen, ein Problem mit der ihnen aufgenötigten Absicherungsintensität haben. 
2. Eine Absicherungsintensität wird politisch-gesetzlich festgelegt und für alle garantiert. Die Individuen dürfen sich darüber hinaus zusätzlich versichern, wenn sie das wünschen und können.

Bei dieser Variante treten jedoch zwei Probleme auf:

Erstens: Wie soll der Umfang der gesetzlichen Basis-Versicherung bestimmt werden? Man könnte diese auf eine Erhaltung und Wiederherstellung der biologischen Kernfunktionalitäten beschränken wollen (vgl. Kersting 2005, S. 148f.). Aber diese Beschränkung löst das zentrale Problem nicht. Denn auch angesichts der Kernfunktionen stellt sich für den Gesetzgeber die Frage, wieviele Ressourcen er zu deren Erhaltung aufwenden soll. Man könnte einräumen, dass es hier gar keine moralisch richtige Entscheidung mehr gibt, dass im politischen Prozeß lediglich die Güterabwägungen einer Mehrheit in Gesetzesform gebracht werden. Aber dann bleibt man mit dem zweiten Problem konfrontiert.

Zweitens: In diesem Modell kann und wird oberhalb des Levels der gesetzlichen Basis-Absicherung das Phänomen auftreten, dass jemand früher sterben muss, weil er sich weitergehende Absicherungen infolge von ungerechtfertigten Vermögensungleichheiten nicht leisten konnte. Und die so verursachte Ungleichheit wird wiederum als Ungerechtigkeit empfunden.

Dieses Modell beschreibt unsere Realität. Wir befinden uns in dieser Situation, obwohl die Bestimmungen des Sozialgesetzbuches jedem lebensbedrohlich Erkrankten alle Behandlungen zugestehen, bei denen eine »nicht ganz entfernt liegende Aussicht auf Heilung « besteht (SGB V, \2, Abs. [1] und [1a]; vgl dazu auch den Beschluss des BVerfG vom 6.12.2005). Eine Beschränkung des Leistungsanspruchs ergibt sich gleichwohl daraus, dass alle zu erbringenden Leistungen unter dem Vorbehalt der Wirtschaftlichkeit stehen (SGB, V, $\mathbb{1} 12$ Wirtschaftlichkeitsgebot). Es hängt deshalb alles davon $\mathrm{ab}$, ob eine lebensverlängernde oder die Lebensqualität verbessernde Maßnahme als wirtschaftlich eingestuft worden ist oder nicht. Diese Einstufung wird zwischen den im Gesundheitswesen arbeitenden Verbänden ausgehandelt und ggf. auch vom behandelnden Arzt oder dem Klinikdirektor vorgenommen. Auf diesen 
Ebenen findet de facto eine vor den Patienten verborgene Priorisierung und Rationierung von Leistungen statt. Wer es sich leisten kann, kann zusätzlich die medizinischen Leistungen kaufen, deren Erfolgswahrscheinlichkeit oder Bedeutung zu gering war, um zu ihrer Anerkennung als >wirtschaftlich < zu führen. Eine zweite Form der Leistungsrationierung ergibt sich daraus, dass etliche hochqualifizierte Ärzte Patienten der gesetzlichen Krankenversicherung (GKV) entweder gar nicht behandeln oder nur einen unverhältnismäßig kleinen Teil ihrer Arbeitszeit auf deren Behandlung verwenden. Dabei ist nicht gewährleistet, dass die schwierigsten Fälle zu den kompetentesten Behandlern gelangen. Es hat dabei keineswegs jeder die gleiche Chance, ein und dieselbe Krankheit zu überleben.

Diese Probleme sollten uns, da wir ja nicht alle verfügbaren Ressourcen in das Gesundheitswesen investieren wollen, geneigt machen, nach einer gerechteren Form der Leistungsbegrenzung zu suchen.

3. Bei dieser Form der Leistungsbegrenzung werden zuerst moralisch akzeptable Absicherungs-Grenzwerte ermittelt. Als Grenzwerte fungieren dabei zwei Absicherungsintensitäten, die individuell bei gerechter Ressourcenverteilung gewählt werden könnten. Die gerechte Verteilung, auf die sich die nachfolgend definierten Grenzwerte beziehen sollen, meint hier wiederum diejenige grundlegende Verteilungsordung, die die Mitglieder einer Gesellschaft politisch für sich angenommen haben.

Der erste Grenzwert entspricht dann derjenigen individuell gewählten (und kollektiv realisierbaren) Absicherungsintensität, die bei gerechter Ressourcenverteilung für die am besten Dastehenden maximal erreichbar wäre. Dieser Wert dient dazu, die Grenze der Höherversicherungserlaubnis in einer Gesellschaft mit ungerechter Einkommensverteilung $\mathrm{zu}$ bestimmen. Ein Höherversicherungsverbot über diesen Wert hinaus beschränkt zwar die Selbstbestimmung der Individuen, es beschränkt sie aber nicht auf eine moralisch unzulässige Weise, weil auch für die höherversicherungswilligen heutigen Reichen unter gerechten Ausgangsbedingungen keine bessere Absicherung erreichbar wäre. Das Verbot einer bes- 
seren Absicherung ist moralisch vertretbar, weil es keinen berechtigten Anspruch gibt, von Ungerechtigkeit profitieren zu können.?

Der zweite Grenzwert entspricht derjenigen Absicherungsintensität, die von den Beziehern des innerhalb des Gerechtigkeitsspektrums geringstmöglichen Einkommens maximal realisiert werden könnte. ${ }^{8}$ Dieser Wert dient dazu, die Versicherungsintensität zu bestimmen, die für jeden Staatsbürger gewährleistet sein muss. Es könnte sich dann niemand beklagen, dass er zu gering versichert ist, weil diese Versicherungsintensität eine ist, die auch unter gerechten Verteilungsbedingungen für viele nicht $\mathrm{zu}$ verbessern wäre.

Auch diese dritte Second-best-Strategie ist mit erheblichen empirischen und philosophischen Problemen konfrontiert:

Zum einen müsste geklärt werden, wie die genannten Grenzwerte bestimmt werden können. Hierzu müßte man zwei Größen kennen: Man müßte a) wissen, für welches Gerechtigkeitsprinzip sich die Bürger eines Staates in freier politischer Selbstbestimmung entscheiden würden. Und man müßte b) wissen, wieviel Prozent der Versicherten unter gerechten Bedingungen wieviel Prozent ihres Einkommens für noch zu spezifizierende medizinische Leistungen ausgeben würden.

Beide Fragen sind empirischer Natur und also philosophisch nicht zu klären. Die Ursache der weitverbreiteten Unzufriedenheit mit dem Zustand unserer Gesellschaft besteht aber genau darin, dass die Frage der Einkommensgerechtigkeit nicht umfassend gestellt wird. Es gibt eine weitgehend einhellige Empörung über die

7 | Natürlich würde es auch bei diesem Höherversicherungsverbot zu einem Krankentourismus der Vermögenderen kommen. Aber der könnte dann nicht mehr - wie unter 1. - als moralisch legitime Reaktion auf eine willkürliche Grenzziehung des Gesetzgebers verteidigt werden.

8 | Mit dem, was jemand tun könnte, können unter moralischer Betrachtung natürlich nur solche Optionen gemeint sein, die jemand realisieren könnte, ohne dafür etwas auf sich zu nehmen, das seinerseits moralisch bedenklich wäre - etwa das Wohnen in einer Wellblechhütte, einen 14-Stunden Arbeitstag oder den Verzicht auf Kinder. 
Höhe von Managergehältern, aber keine klare Vorstellung davon, ab wann ein Gehalt moralisch anstößig ist. Und dasselbe gilt für die geringsten Einkommen. Es gibt einen recht breiten politischen Konsens über Mindesteinkommen. Aber ob die so bestimmten Einkommen auch gerecht sind, ist damit nicht beantwortet. Und eben weil die Frage nach der Gerechtigkeit in der nötigen Klarheit weder gestellt noch beantwortet wird, wird allen Bürgern zum Trost und zur Ruhigstellung die Partizipation an einer staatlich garantierten Basisversicherung geboten. Aber diese Basisabsicherung verliert ihre sedierende Wirkung, je mehr deren Leistungsumfang schrumpft und je offenkundiger die Unterschiede zwischen Basisversorgung und medizinisch sinnvoller Vollversorgung zutage treten. Wir sind Zeugen eines Prozesses, in dem die basale Ungerechtigkeit der Gesellschaft in immer mehr Bereiche des Lebens ausstrahlt - in den Zugang zu medizinischen Leistungen ebenso wie in den Zugang zu guten Schulen und Universitäten. In den USA werden mittlerweile den Vermögenderen in privat betriebenen Gefängnissen bessere Zellen gegen Bezahlung angeboten.

Zum anderen wäre auch zu klären, anhand welcher Spezifizierungskategorien Personen über die von ihnen bevorzugte $\mathrm{Ab}$ sicherungsintensität überhaupt nachdenken könnten. Man müsste dazu wissen, welche Verbesserung einer Erkrankungssituation mit wieviel zusätzlichen Ressourcen erreichbar wäre. Das sind sehr komplexe und stets sich neu herstellende Konstellationen. ${ }^{9}$ Würde man etwa den Einsatz eines neuen Medikaments finanzieren wollen, dass X Euro kostet und das Leben der damit Behandelten um Y Monate verlängert oder auf die-und-die Weise verbessert? Für diese Entscheidungen kann man vermutlich nur eine für alle vorkommenden Fälle gültige Kosten-Nutzen-Relation (KNR) als Grenzwert festlegen. ${ }^{10}$ (Etwa: Man ist bereit, sich für Leistungen zu versichern,

9 | Vergleiche die in diesem Band geschilderten Versuche Berechnungsformeln zu entwickeln.

10 | Hier entsteht jedoch das Problem, den "Nutzen" genau zu definieren. Die in der Debatte verwendeten Größen wie QALY, DALY, HYE usw. bilden 
bei denen die Kosten für jeden gewonnenen Lebenstag nicht höher sind als 100 Euro.) Zugleich wird man aber eine Vorstellung davon haben, wieviel Prozent seines Einkommens man insgesamt für medizinische Absicherungen ausgeben will. Die Entscheidung wird durch eine schwer analysierbare Interferenz dieser beiden Perspektiven erfolgen:

- Weder wird man sagen »Ich will mich für alle Leistungen versichern, bei denen die KNR nicht größer ist als $\mathrm{X}$ « - ohne zu wissen, wieviel Prozent des Einkommens diese Versicherung verbrauchen würde.

- Noch wird man einfach sagen können »Ich bin bereit, Y Prozent meines Einkommens für die Krankenversicherung zu investieren « - ohne zu wissen, welche Optionen (mit welchen KNR) man damit preisgibt.

Schließlich müsste, drittens, geklärt werden, in welchem Umfang es möglich ist, Krankheiten als selbstverschuldet zu identifizieren. Daran schließt sich die Frage an, inwieweit es sinnvoll ist, die Behandlung dieser Krankheiten aus dem allgemeinen Versicherungskatalog herauszunehmen und evtl. durch Zusatzversicherungen versicherbar zu machen.

Viertens ist $\mathrm{zu}$ fragen, wie die Versicherungen finanziert werden? Hier muss man berücksichtigen, dass - voraussetzungsgemäß - die Beiträge heute unter insgesamt ungerechten Verteilungsbedingungen zu erbringen sind. Es wäre deshalb legitim, wenn eine Regierung diese Ungererechtigkeit durch einkommensrelative Beiträge so weit wie möglich (d.h. politisch durchsetzbar) zu mildern versucht. Hier wäre, wie bei der Steuer, auch eine prozentuale Progression vertretbar. Die Beiträge zur Krankenversicherung würden so ein Instrument gerechtfertigter Umverteilung. Dagegen ist die heute etablierte Beitrags-Kappungsgrenze mit Sicherheit ein Ver-

jeweils ein anderes Nutzen-Verständnis ab und sind umstritten. Die Forderung einer KNR ist daher wesentlich komplexer. 
stoß gegen jedes Prinzip der Einkommensverteilung, das realistischerweise von einer Mehrheit der Bevölkerung als gerecht anerkannt werden würde.

Und letztlich wäre zu klären, was mit Individuen ist, die unter gerechten Rahmenbedingungen eine geringere Versicherungsintensität als den unteren Grenzwert wählen würden. Denen würde selbst mit der Versicherung zum unteren Grenzwert eine Absicherungsintensität aufgenötigt, die nicht ihren Präferenzen entspricht. Dürfen die sich schlechter versichern? Ich denke, das sollte in den Grenzen eines vertretbaren Paternalismus, der eine solche Unterversicherung notfalls verhindern könnte, möglich sein. Vertretbar ist ein Paternalismus, der a) erfahrungsbasiert Individuen vor Situationen des Bereuens schützt (»Jetzt, wo ich krank bin und nicht besser behandelt werde, kann ich erst einschätzen, was im Leben wirklich wichtig ist. Ich wollte, ich hätte mich besser versichert.«), der b) vorhersehbare Externalitäten (Kriminalität usw.) verhindert und der c) die Unterschreitung des Grenzwertes nur mündigen Bürgern erlaubt. Heranwachsende und geistig Behinderte sind zum unteren Grenzwert zu versichern.

\section{FazIT}

Mal angenommen, das Grenzwert-Modell (mit einem Unter- und einem Obergrenzwert) ist handhabbar, dann kann man mit seiner Hilfe die untere und für jeden Staatsbürger zu garantierende Versicherungsintensität bestimmen. Dieser Intensität würde eine bestimmte Kosten-Nutzen-Relation entsprechen. Die Finanzierung dieser Absicherung dürfte der Staat - unter Berücksichtigung der gerade erwähnten Ausnahmen und Beitragsstaffelungen - jedem Bürger auferlegen. Das erlaubt zugleich die Bestimmung der moralisch notwendigen Zuschüsse des Gesetzgebers an Personen, die diese Absicherungsintensität heute mit eigenen Mitteln nicht erreichen können. Die dann ebenfalls mögliche Bestimmung der höchsten Absicherungsintensität - sowie der korrespondierenden KNR 
- bestimmt schließlich den Rahmen, innerhalb dessen die Individuen ihre Versicherungsform wählen dürfen. Das wäre eine Verbesserung gegenüber einer nur von Machtverhältnissen oder Intuitionen gesteuerten politischen Bestimmung des Budgets. Es bliebe eine umständliche Second-best-Strategie und zudem eine lediglich lokale Verminderung der Ungerechtigkeit in einer hinsichtlich ihrer Ressourcenverteilung ungerechten Gesellschaft. Aber solange eine durchgreifende Verminderung der gesamtgesellschaftlichen Ungerechtigkeit nicht erreicht ist, bleibt diese Organisation des Krankenversicherungswesens die einzige Form, um jedem Bürger eine faire Selbstbestimmung hinsichtlich der Frage zu ermöglichen, wie er sich gegen solche Bedrohungen seines Lebens schützen will.

In diesem System wird es - ebenso wie in einer Krankenversichung in gerechten Rahmenbedingungen - drei Formen von Rationierung geben:

1. Leistungen mit KNRs, die den oberen Grenzwert übersteigen, werden gar nicht angeboten, auch wenn sie technisch oder pharmakologisch möglich wären. Solche KNR-Limitierungen werden zugleich steuernd auf die Medizinforschung wirken. Es ist für gewinnorientierte Unternehmen sinnlos, Therapiemöglichkeiten zu erforschen, von denen erkennbar ist, dass niemand sie wird bezahlen wollen.

2. Die Versicherten erhalten über die Basisabsicherung hinaus lediglich jene Leistungen, die zum Leistungsangebot der von ihnen gewählten Versicherung gehören.

3. Es darf Versicherungen geben, die die jeweils angebotenen Leistungen nicht denen zur Verfügung stellt, bei denen die KNR altersbedingt einen bestimmten Wert überschreitet. Aus der Perspektive der Individuen kann es eine akzeptable Altersdiskriminierung geben, denn viele werden ihre Ressourcen eben primär für Absicherungen verwenden wollen, von denen sie im Ernstfall länger profitieren würden. ${ }^{11}$ Es ist für viele zentrale Lebensprojekte (Kinder großziehen, berufliche Entwicklung) zudem sehr viel wichtiger,

11 | Vgl. auch die Überlegungen von Norman Daniels (1985). 
überhaupt ein Alter von 60 zu erreichen, als gegen Krankheiten versichert zu sein, die einen erst mit 70 Jahren treffen können.

All diese Abwägungen zur Wahl der gewünschen Versicherungsintensität sind für die Versicherungssuchenden sehr kompliziert. Wer kann sich schon vorstellen, wie schlimm es ist, mit 30, 40 oder 60 Jahren an Magenkrebs zu erkranken und dann abwägen, was er zur Finanzierung welcher der möglichen Therapien jeweils aufzuwenden bereit wäre? Die allgemeine Form solcher Fragen wäre: Was ist die für mich richtige Vorsorge für eine Krankheit mit der Beschädigungsqualität $\mathrm{X}$, die mich mit einer Wahrscheinlichkeit von Y Prozent heimsuchen kann. Bei Lichte besehen überfordern solche Fragen jeden. Wir können nur mit vagen Intuitionen operieren. Das ist unbefriedigend, aber was ist die Alternative zur individuellen Wahl? Sollen die Rationierungsentscheidungen nach dem Kriterium der sogenannten Wirtschaftlichkeit weiterhin von angeblichen Experten hinter verschlossenen Türen ausgehandelt und für alle verbindlich gemacht werden? Wissen die denn besser, was in meinem, in unserem Interesse ist? Das steht nicht zu vermuten. Wie sollten sie es auch besser wissen können? So bleibt uns die Qual der individuellen Wahl nicht erspart - eine Wahl bei unvollständigem Wissen und mit unzureichenden kognitiven Fähigkeiten. Wie meistens im Leben.

Eine solche Umgestaltung des Gesundheitswesens wird vermutlich auf ähnlich starke Widerstände stoßen wie jeder ernsthafte Versuch, die Verteilungsungerechtigkeit zu beseitigen. Dies vor allem deshalb, weil mit dem hier vorgeschlagenen Modell eine Umkehrung der Perspektive verbunden ist. Es ginge nicht länger an, dass sich Experten fragen, wie ein Gesundheitsbudget an die Bürger verteilt werden soll. Vielmehr wären nun die Bürger selbst die bestimmende Kraft. Die Bürger, die sich individuell fragen müssen, wieviel sie für welche Art von medizinischer Dienstleistung auszugeben bereit sind. Bürger, die im selben Atemzug einsehen müssten, dass sie sich nicht auf ein irgendwie gegebenes Recht auf Gesundheitsfürsorge berufen können, sondern nur auf eine von ih- 
nen selbst ins Leben gerufene und finanzierte Absicherung gegen elementare Risiken. Abhängig von diesen Entscheidungen müssten Ärzte und Pharmafirmen ihre Angebote strukturieren und begrenzen. Dabei brauchte sich niemand mehr moralisch zu empören oder zu grämen, wenn er eine durchaus mögliche Leistung nicht erhält oder erbringt, weil die (zu diesem Preis) entweder niemand oder die Mitglieder bestimmter Versicherungsklassen nicht haben wollen. Solche Bürger müssten sich auch mit den folgenden drei Problemen beschäftigen, zu deren Lösung ich hier nur noch ein paar Anregungen geben möchte:

Erstens: Eine Gesellschaft muss sich - im Idealfall durch demokratische Deliberation - auf ein Aggregationsprinzip oder auf eine Kombination derselben festlegen. Warum soll sie dann nicht diesem Prinzip entsprechend auch hinsichtlich der medizinischen Fürsorge eine für alle verbindliche Absicherungsintensität politisch festlegen? ${ }^{12}$

Angenommen, eine Gesellschaft hat sich mit 90-prozentiger Mehrheit für die Akzeptanz des Maximin-Prinzips entschieden, wäre es dann nicht konsequent, wenn sie auch den Medizinsektor nach diesem Prinzip organisierte? Das würde im Fall des Maximin-

12 | Wenn man die Mittelverteilung im Gesundheitswesen direkt von den möglichen Moralprinzipien abhängig macht, gilt: Eine Rationierung ist nur legitim, wenn sie sich von dem in einer Gesellschaft anerkannten Aggregationsprinzip her rechtfertigen lässt. Für das utilitaristische Aggregationsprinzip liegt die Orientierung an der Gesamtsumme der QALYS nahe. Bei der Orientierung an Gleichheit stellt sich die Frage, ob eine Gleichheit hinsichtlich des durch medizinische Maßnahmen Hinzuzugewinnenden oder hinsichtlich des durch die Maßnahmen hergestellten Endzustandes gemeint ist. Beim Optimierungsprinzip geht es um die bestmögliche Einrichtung der schlechtestmöglichen Position. Das wird zu einer Priorisierung von Maßnahmen führen, die das Leben junger Menschen verlängern oder verbessern. Dabei müsste man ggf. große Nutzenverluste bei bereits (von Natur) Bessergestellten in Kauf nehmen (Therapie von Beta Thalassämie bei wenigen versus Therapie von Prostatakrebs bei vielen). 
Prinzips etwa dazu führen, dass Krankheiten, die Menschen in jungen Jahren töten können - etwa Beta-Thalassämie -, prioritär erforscht und behandelt werden und zwar auch dann, wenn daran nur wenige erkranken und die Behandlung vergleichsweise sehr teuer ist. Das würde sich aus dem Maximin-Prinzip ergeben, weil die in jungen Jahren von einer Krankheit Getöteten eben zu den Schlechtestgestellten gehören - sie werden unbehandelt mehr verlieren als jeder verlieren kann, der schon das Erwachsenenalter erreicht hat.

Das scheint jedoch eine verkürzte Perspektive zu sein. Es wird der moralischen Perspektive nicht gerecht, wenn man den Leidenden nur im Leidenszeitpunkt betrachtet. Man muss die Individuen vielmehr in ihrem Bezug auf ihr Leben als Ganzes wahrnehmen. D.h. man muss sie zuerst fragen, wieviel Schutz vor den bekannten Lebensrisiken sie denn haben wollen, d.h. mit welcher Absicherungsintensität sie meinen, so gut wie möglich dazustehen. Die Individuen müssen selbst entscheiden, mit Inkaufnahme welcher ggf. auch genetisch bedingten Lebensrisiken sie insgesamt am besten leben. Ein Staat, der mit Steuergeldern weitere Verbesserungen würde herbeiführen wollen, täte gar nicht mehr das, was im Interesse seiner Bürger ist. Auch wenn die Bürger eine Gerechtigkeitsvorstellung politisch akzeptiert haben, ist damit noch nicht entschieden, welche Güterordnung und damit auch welche Absicherungsintensität sie als für sich optimal befürworten. Auch innerhalb eines jeden Aggregationsprinzips gibt es noch die individuell divergierenden Gütergewichtungen.

Dem allgemeinen Gerechtigkeits-Prinzip der Gesellschaft tut der Staat dadurch genüge, dass er die Schlechtestgestellten hinsichtlich der Universalressource Geld so gut wie möglich stellt. Und auf dieser Basis haben die Individuen dann Entscheidungen über Absicherungsintensitäten getroffen, bei denen auch solche seltenen Krankheiten berücksichtigt werden konnten.

Zweitens: Wie geht man mit angeborenen Erkrankungen oder Risikofaktoren um? Diese Erkrankungsrisiken und deren Behandlungskosten sind bei der Wahl der Versicherungsintensität mit 
zu berücksichtigen. Man muss sich ja dabei auch zu der Tatsache verhalten, dass einen diese Krankheit treffen könnte. Idealerweise müssten die Individuen ihre Entscheidung hinsichtlich der Absicherungsintensität unparteiisch, also ohne Kenntnis ihrer realen gesundheitlichen Konstitution treffen. Das ist natürlich nicht vollständig möglich. In dem Alter, in dem man rational über die Art der Krankenversicherung nachdenken kann, weiß man bereits eine Menge über die eigene Konstitution. Wenn es den Versicherern erlaubt wäre, nach solchen Daten zu fragen und denen entsprechend ein Versicherungsangebot zu kalkulieren, dann würden Personen ggf. lebenslang für ihre Natur bestraft oder belohnt. Das würde man aus einer moralisch-unparteiischen Perspektive nicht gutheißen. Dieses Problem lässt sich dadurch vermeiden, dass alle wählbaren Versicherungspakete die bis zum Zeitpunkt der Entscheidung erkennbaren Erkrankungen enthalten und zu einem Tarif versichern müssen, der ihrer statistischen Häufigkeit entspricht.

Drittens: Führt das nicht zu einer inakzeptablen Diskriminierung infolge von Seltenheit? Zur Behandlung seltener Krankheiten wird von gewinnorientierten Unternehmen naturgemäß wenig geforscht. So bleibt manches unbehandelbar, was vermutlich behandelbar wäre, wenn es nur mehr Erkrankte gäbe. Und selbst wenn eine Behandlung möglich wäre, könnte diese infolge der geringen Zahl der Fälle pro Kopf sehr teuer werden (kleine Produktionschargen usw.). Dieser Umstand könnte die Behandlungskosten über den ansonsten akzeptierten KNR-Wert treiben, so dass die Behandlung nicht versicherbar wäre und also unterbliebe. Ist das akzeptabel oder nicht? Auch diese Frage müsste aus der unparteiischen Perspektive entschieden werden. Aus dieser Perspektive würde man vermutlich befürworten, dass niemand höhere Gesundheitskosten infolge von (genetic) bad luck tragen muss. D.h. man könnte sich für die ggf. anfallenden Behandlungskosten versichern (oder eben auch nicht), aber aus den Kosten müsste zuvor die Verteuerung infolge von Seltenheit herausgerechnet und der Zusatzbetrag staatlich getragen werden. 


\section{ZItIERTE UND WeITERFüHRENDE LITERATUR}

Ach, J.S.; Schöne-Seifert, B.; Buyx, A.M. (Hg.) (2006): Gerecht behandelt? Rationierung und Priorisierung im Gesundheitswesen. Münster: Mentis.

Beckmann, J.P. (2009): Ethische Herausforderungen der modernen Medizin. Freiburg: Alber.

Buddha (1993): Reden des Buddha. Stuttgart: Reclam.

Daniels, N. (1985): Just Health Care. Cambridge: University Press.

Dworkin, R. (1981): What is Equality? Part 2: Equality of Resources. Philosophy and Public Affairs 10: 283-345.

Gethmann-Siefert, A.; Thiele, F. (Hg.) (2008): Ökonomie und Medizinethik. München: Wilhelm Fink.

Kersting, W. (2005): Kritik der Gleichheit. Weilerswist-Metternich: Velbrück.

Lübbe, W. (Hg.) (2003): Tödliche Entscheidung. Allokation von Leben und Tod in Zwangslagen. Münster: Mentis.

Marckmann, G.; Liening, P.; Wiesing, U. (Hg.) (2003): Gerechte Gesundheitsversorgung. Ethische Grundpositionen zur Mittelverteilung im Gesundheitswesen. Stuttgart: Schattauer.

Sass, H.M. (Hg.) (1994): Medizin und Ethik. Stuttgart: Reclam.

Schöne-Seifert, B. (2007): Grundlagen der Medizinethik. Stuttgart: Kröner.

Wallner, J. (2004): Ethik im Gesundheitssystem. Stuttgart: UTB.

Wiesing, U.; Ach, J.S.; Bormuth, M. (Hg.) (2000): Ethik in der Medizin. Ein Reader. Stuttgart: Reclam. 



\section{Anerkennung des Individuums oder des Kollektivs?}

Sozialhygienische Zuteilungskriterien im

Gesundheitswesen bei Alfred Grotjahn,

Max Mosse und Gustav Tugendreich im Vergleich zur heutigen Sozialepidemiologie

Sebastian Kessler

\section{Einleitung: Soziale Ungleichheit Und deR Wert VON KRANKEn IN DER SOZIALEPIDEMIOLOGIE UND IHREN VORLÄUFERN}

Der Zusammenhang von Armut und Krankheit beschäftigt Medizin und Wissenschaft bereits seit einiger Zeit. ${ }^{1}$ Auch in neueren sozialepidemiologischen Arbeiten steht die Frage nach dem Einfluss von gesellschaftlichen Variablen auf Morbidität und Mortalität im Zentrum des Erkenntnisinteresses. Dabei handelt es sich schon längst nicht mehr um einen kleinen Seitenzweig der Epidemiologie. Andreas Mielck trug allein für den Zeitraum zwischen 1950 und 1999 insgesamt 441 deutschsprachige Publikationen zusammen, die das übergeordnete Thema soziale Bedingtheit von Krankheit und Gesundheit aus einer wissenschaftlichen Perspektive in den Blick nehmen (vgl. Mielck 2000, S. 67). Seitdem hat die Veröffent-

1 I Ich danke Uta Bittner, Heiner Fangerau und Kathrin Dengler für ihre Anmerkungen, Vorschläge und Ideen zu diesem Text. 
lichungsrate von Studien über den Einfluss sozialer Faktoren auf beispielsweise kardiovaskuläre Krankheiten (siehe exemplarisch Puska und Keller 2004), Übergewicht im Kindesalter (siehe exemplarisch Kolip 2004) oder die Zahngesundheit (siehe exemplarisch Heinrich-Weltzien et al. 2007) eher zu- als abgenommen.

Die junge Sozialepidemiologie formierte sich in Deutschland als wissenschaftliches Fach vor allem nach der Publikation des richtungsweisenden Black-Reports (Townsend 1982). In deutschsprachigen Texten wird auf der Suche nach einer Tradition aber immer wieder auch auf die Werke von Alfred Grotjahn (1869-1931) (Grotjahn 1977 [1923]), sowie von Max Mosse (1873-1936) und Gustav Tugendreich (1876-1948) (Mosse und Tugendreich 1977[1913]) verwiesen. Beispielsweise schreibt Manfred Pflanz, damaliger Direktor der Abteilung Epidemiologie und Sozialmedizin der Medizinischen Hochschule Hannover, im Vorwort des Reprints von 1977, der Band von Mosse und Tugendreich sei kein Museumsstück. Im Vergleich stelle man fest,

"daß unsere heutige Sozialmedizin, wiederentstanden lange Jahre nach dem 2. Weltkrieg, noch längst nicht wieder den Stand des Wissens und vor allem des Ansetzens an soziale Probleme erreicht hat, der damals selbstverständlich war. Wir sind erst angelangt im Mittelalter und bei der Auffassung, alles Kranksein ist der Sünde Sold, der Sünden des Essens, Rauchens und Trinkens. Soziale Faktoren werden entweder abgewertet oder übermäßig gezeichnet. Arme Menschen, Leute in schlechten Wohnungen und harten Berufen werden heute als Randgruppen abqualifiziert, während sie von den Autoren des Mosse/Tugendreich liebevoll betrachtet werden, als Gruppe, deren natürlicher Anwalt der Arzt ist" (Mosse und Tugendreich 1977[1913], Vorwort Manfred Pflanz).

Die Adelung durch die Zitierung genau dieser Textstelle findet sich in der Folge auch in einigen der heutigen Forschungsstände 
wieder. ${ }^{2}$ So werden die »Arbeiten von Grotjahn (1923) und Mosse u. Tugendreich (1913)« (Schaefer 1996, S. 443) ohne weitere Einschränkung hintereinander weg genannt und als »bahnbrechend « (Schaefer 1996, S. 443) bezeichnet. ${ }^{3}$ Auch in den Überlegungen zur »Entwicklung eines Modells zur Erklärung gesundheitlicher Ungleichheit« (Elkeles und Mielck 1997) wird an die beiden deutschen Autoren zu Beginn des 20. Jahrhunderts erinnert. Die Frage des Einflusses sozialer Faktoren auf Morbidität und Mortalität sei nicht neu. »Die ssoziale Medizin< [habe] sich in Deutschland, insbesondere nach der Jahrhundertwende bis zum Beginn des 1. Weltkriegs, intensiv mit ihr beschäftigt« (Elkeles und Mielck 1997, S. 137). Das Bedürfnis der Erfindung einer eigenen Geschichte geht schließlich sogar so weit, die Tradition der

"sozialen Medizin seit J. P. Frank (1745 -1821). S. Neumann (1819-1908), R. Virchow (1821 -1902). A. Grotjahn (1869 -1931), Mosse und Tugendreich (2). sowie A. Gottstein (1857 -1941)“ (Gostomzyk und Schaefer 1998, S. 4) ${ }^{4}$

als ab 1933 unterbrochen zu bezeichnen. Demnach sei es bedauerlich, dass sich die Neuanfänger in dem Forschungsbereich ab 1961 nicht mit den alten Studien auseinandergesetzt haben. Der Verlust des Wissens über die Sozialhygiene sei auf die starke Neuausrichtung in der Medizin ab der Machtübertragung an die Nationalsozialisten zurückzuführen. Nach der Zeit des Nationalsozialismus, so muss man in der Konsequenz

2 | Vgl. zum Beispiel Mielck 1994, S. 51; Brand und Schmacke 1998; Mielck 2000, S. 127. In späteren Forschungsständen wird auch nicht mehr auf die Originalwerke verwiesen, sondern auf die medizingeschichtliche Darstellung in den Forschungsständen von Kollegen. Vgl. zum Beispiel Trojan 2001, S. 46.

3 | Auch andernorts finden sich entsprechende Verweise auf Grotjahn (vgl. exemplarisch Bardehle 2001, S. 342) sowie Mosse und Tugendreich (vgl. exemplarisch Swart 2001, S. 330)

4 | Vgl. auch Gostomzyk 2001, S. 220-223. 
weiter vermuten, wäre dann das >vor-nationalsozialistische< Wissen um Armut und Krankheit in Vergessenheit geraten. Dabei wird insbesondere Grotjahns »Verhältnis zur Eugenik« (Mielck 1994, S. 38) früh problematisiert. Nicht ganz zehn Jahre vor der Einreihung von Grotjahn, sowie Mosse und Tugendreich in die wissenschaftliche Tradition von Frank bis Gottstein wird von Kaspari festgehalten, dass sich

"die Grundlagen von Grotjahns Eugenik als spezifisches, aber zwanglos in den Rahmen der generellen rassenhygienischen Strömung jener Zeit einzuordnendes Konzept“ (Kaspari 1989a, S. 314-315)

erweisen würden. Auch die Warnung »Vorsicht vor Alfred Grotjahn!« des Direktors des Instituts für Medizinische Soziologie in Frankfurt, Hans-Ulrich Deppe, war zu diesem Zeitpunkt bereits seit 15 Jahren publiziert (vgl. Deppe 1983). ${ }^{5}$ Der Sozialdemokrat Alfred Grotjahn, der nicht nur ab 1920 Ordinarius für Sozialhygiene an der Universität Berlin, sondern auch von 1921-1924 Mitglied des Reichtages war und von 1925-1928 an der hygienischen Sektion des Völkerbunds in Genf mitarbeitete (vgl. Koppitz und Labisch 2007), strebte nach einer >guten < Bevölkerungspolitik in der Weimarer Republik. Eugenische Überlegungen waren ein Kern-

5 | Es darf nicht unterschlagen werden, dass diese sich bereits 1994 findende Problematisierung der Traditionsbeziehung zu den frühen Autoren immer wieder aufgegriffen wird (Gardemann 2001). Es wird zum Beispiel erklärt, dass die Diskussion um den Einfluss von Klima und Rasse in den alten Werken Teil der wilhelminischen Zeit war, und herausgestellt, dass sich Mosse und Tugendreich gegenüber rassenbiologischen Ausleseverfahren kritisch geäußert haben. Die Gesundheitsfürsorge der Weimarer Republik fuße jedoch "im Gedankengut der Sozialhygiene“ (Gardemann 2001, S. 279). In neueren Publikationen finden sich außerdem informierte Einordnungen von Alfred Grotjahns Ansatz in die "Entwicklung von Konzepten und Methoden der Prävention “ (Rosenbrock und Kümpers 2006, S. 414), in der auch seine eugenischen Überlegungen differenziert in Frage gestellt werden. 
bestandteil seines Denkgebäudes über Ungleichheit und Krankheit. Der Internist Mosse und der Pädiater Tugendreich (Seidler 2008, S. 26) richteten ihr Hauptaugenmerk auf die soziale Lage. Auch sie schlossen den Nutzen von den von ihnen als vererbungshygienisch klassifizierten Ansätzen (vgl. Mosse und Tugendreich 1977[1913], S. 21) nicht aus. Für Grotjahn ist das Allgemeinziel der Sozialhygiene »die Fernhaltung der gesundheitlichen Schädigungen in negativer und die Vervollkommnung der Körperkonstitution in positiver Hinsicht« (Grotjahn 1977 [1923], S. 8). Mosse und Tugendreich möchten klären, inwiefern sich allgemeine soziale Einflussfaktoren auf Krankheit auswirken oder wie sich negative soziale Einflüsse beseitigen bzw. vermeiden lassen, um die allgemeine Gesundheit in der Gesellschaft zu stärken. Offensichtlich liegen hier Anknüpfungspunkte für die heutige Literatur, die auf die beiden Werke als historische Vorgänger der eigenen Forschung verweist (siehe oben). Wie in der Forschung Gesunden und Kranken Wert zugeteilt wird, hat sich hundert Jahre nach dem Erscheinen der Werke von Grotjahn, Mosse und Tugendreich zwar sehr verändert, dennoch lohnt sich eine Rückschau auf diese Arbeiten, um Einblicke in die Konstruktion sozialer Wertschätzungen und die Zuteilungskriterien im Gesundheitswesen aus unterschiedlichen sozialen Perspektiven auf Krankheit zu gewinnen.

Diese scheint umso notwendiger, da die Sozialepidemiologie sich hier in eine Traditionslinie stellt, die aus heutiger Sicht nicht vollständig unproblematisch erscheint. In der unter dem Eindruck der eugenischen Bewegung stehenden Literatur aus den ersten Jahrzehnten des 20. Jahrhunderts kommen andere Formen der Anerkennung von >krank oder sgesund zum Tragen. Während in der Eugenik ein Teil der Untersuchungspopulation missachtet wurde und sich ein Bestreben abzeichnete, nur als wertvoll anerkannte Individuen in eine idealisierte Gesellschaftsutopie einzubeziehen, muss die Sozialepidemiologie die Frage stellen, inwiefern ein unproblematisierter Verweis auf frühere Forschungen der Sozialhygiene heutige Normvorstellungen unterwandern kann. Der hier zugrundeliegenden Hypothese folgend bringt die heutige Sozialepi- 
demiologie dem Einzelnen im Gesundheitswesen ein höheres Maß an Anerkennung entgegen als ihre (selbstgewählten) Vorläufer.

Im Folgenden möchte ich daher an exemplarisch ausgewählter Literatur Zuteilungskriterien der Achtung und Missachtung ausarbeiten, die sich entweder am Individuum oder Kollektiv orientieren. ${ }^{6}$ Dafür wird zunächst dargestellt, wie Anerkennungsphänomene wissenschaftliche Überlegungen zu Gesundheit und Krankheit mit impliziten Normen der Zuteilung verknüpfen. Anschließend wird die Anerkennung des Wertes individueller Gesundheit und Krankheit bei Grotjahn sowie bei Mosse und Tugendreich herausgearbeitet. ${ }^{7}$ Wie Heiner Fangerau bereits gezeigt hat waren »Prozesse der Anerkennung und Selbstkonstitution zentral [...] für die eugenische Diskussion und Wirkmächtigkeit ihrer Argumente« (Fangerau 2013, S. 84) im Zeitraum zwischen 1890 und 1945. Diese werden danach mit jenen sozialepidemiologischen Arbeiten verglichen, die sich in ihren Forschungsständen explizit auf die frühen Werke beziehen. Hier soll eine Forschungsfrage um die wissenschaftliche Achtung des Individuums respektive des Kollektivs in der Präventionsforschung entwickelt und exemplarisch getestet werden. Trägt der induktiv erarbeitete Vergleich von Unterschieden der Anerkennung von Gesundheit und Krankheit zwischen den ausgewählten

6 | Hinweise auf historische Veränderungen in der Gesundheitspolitik zwischen kollektiver und individueller Verortung finden sich auch an anderer Stelle. Siehe hierfür beispielsweise: Lengwiler/Madarász 2010, S. 15-16.

7 | Sowohl zu den drei genannten Autoren, als auch zur Sozialhygiene und im weiteren Sinne auch zur Rassenhygiene existiert eine ganze Reihe von Ausarbeitungen, Analysen und Untersuchungen. Da in diesem Text der Fokus auf dem historisch kontingenten Wandel von Anerkennungsstrukturen und Gerechtigkeitsverständnissen liegt, kommen diese Arbeiten hier leider nicht zu der innen gebührenden Geltung. Siehe exemplarisch: Thomann 1979; Weindling 1984; Weindling 1985; Weindling 1987a; Weindling 1987b; Kaspari 1989a; Kaspari 1989b; Labisch 1992; Weingart, Kroll, Bayertz 1992; Fangerau 2001; Mildenberger 2002; Roelcke 2002; Satzinger 2002. 
Arbeiten aus beiden Epochen, soll abschließend ein Ausblick gegeben werden, wie die Differenzierung zwischen der Achtung individueller und kollektiver Normen weiter historisch vergleichend erforscht werden kann. Zunächst soll aber auf den Zusammenhang von Anerkennungsprozessen von Gesundheit und Krankheit mit impliziten Normen eingegangen werden.

\subsection{Anerkennung und Selbstkonstitution: Einfluss wissenschaftlicher Erkenntnis auf die soziale Wertschätzung}

Anerkennung als vollständige Persönlichkeit, aber auch als Teil einer Gemeinschaft, ist Grundlage der Ausformung des eigenen Selbst. Da Wissenschaft heute mit Erkenntnis identifiziert wird (vgl. Habermas 2001, S. 13), spielen moderne Wissenschaften eine besondere Rolle für die Art und Weise, wie in der Gesellschaft Anerkennung zugeteilt wird. So können Personen, die aufgrund einer wissenschaftlichen Rechtfertigung als wertvoll gelten, in die Gemeinschaft eingeschlossen werden. Menschen jedoch, die beispielsweise in der Sozialhygiene wegen $>$ degeneriertem Erbgut $<$ nicht als vollständige Persönlichkeit anerkannt werden, sind in Deutschland entlang dieser Logik mit externalisierter Legitimation aus der Gesellschaft ausgeschlossen worden.

Da das Selbst sich immer »aus der Perspektive seines Gegenübers« (Honneth 2008, S. 119) wahrnimmt, hat Missachtung nicht nur eine Auswirkung auf die Handlung der Gruppe, sondern vermittelt immer auch auf das Individuum. Wird Anerkennung verweigert und auch nicht erfolgreich erkämpft, kann in Folge eines Ausschlusses aus der Gemeinschaft der >Selbst-Wert< des oder der Missachteten sinken. Das dialektische Paar von Anerkennung und Selbstkonstitution ist daher grundlegend, sowohl für die individuelle Persönlichkeitsentwicklung, als auch für Ausformungen kollek- 
tiver Handlungen und Partizipation in der Gesellschaft. ${ }^{8}$ In einem »Kampf um Anerkennung « (Honneth 2008) würden nach Axel Honneth Selbstvertrauen, Selbstachtung und Selbstwertgefühl erstritten, erteilt oder verweigert. Dabei unterscheidet Honneth mit der Anerkennung als eigenständiges Subjekt in der Liebe, der Achtung als Rechtssubjekt und der sozialen Wertschätzung als moralische Person zwischen einer individuellen, einer legalen und einer gesellschaftlichen Ebene. Szientistische Einschätzungen zur Bedeutung einer Person für die Fortpflanzung und gesellschaftliche Reproduktion (etwa weil sie an einer Erbkrankheit leidet oder nicht), können der betroffenen Person soziale Wertschätzung verweigern oder zugestehen. Ähnlich wie es Judith Butler für die Ausbildung und Zuweisung von Geschlechterkategorien zeigt, können historisch kontingente, wissenschaftliche Diagnosekriterien Menschen in unterschiedliche Gruppen, Kategorien und Kohorten einteilen. ${ }^{9}$ In der Folge ist es daher möglich, dass den Menschen, die anhand angeblich >harter< wissenschaftlicher Kriterien in unterschiedliche Gruppen eingeteilt wurden, ein unterschiedlicher sozialer Wert zugestanden wird.

8 Siehe für sozialphilosophische Arbeiten zu Anerkennung: Honneth 2008; Fraser et al. 2003; Honneth 2002. Nach Honneth liegt die Grundlage für Teilhabe an und in der Gesellschaft in der Anerkennung des Subjektes. Konstitution des Selbst und Anerkennung seien demnach in einem Aushandlungsprozess miteinander verbunden, für den Honneth auch die Metapher des "Kampfes um Anerkennung" gebraucht (siehe auch: Habermas 1999; Habermas 1991; Honneth 1990; Honneth 2000).

9 | Nach Judith Butler vollzieht sich die Ausbildung des sozialen Geschlechts mit der ärztlichen Untersuchung eines Kleinkindes. Dieses werde in der Untersuchung "von einem ıesı zu einer ssie` oder einem ıerı (Butler 1997, S. 29). Dem Körper werde so ein weibliches Geschlecht zugeordnet. Die soziale Kategorisierung in unterschiedliche gesellschaftliche Gruppen durch die wissenschaftliche Forschung erscheint mir hier vergleichbar. 


\section{Vom Primat der optimalen medizinischen VERSORGUNG DES IVOLKSKÖRPERS、 \\ - EXEMPLARISCHE ANALYSE DES KRANKHEITSVERSTÄNDNISSES BEI GROTJAHN UND MOSSE/Tugendreich}

Dem oben skizzierten Plan folgend werden im Weiteren die Anerkennungsstrukturen von Gesundheit und Krankheit bei Grotjahn sowie Mosse und Tugendreich herausgearbeitet. ${ }^{10}$ Im Vergleich lassen sich einige Gemeinsamkeiten zwischen den beiden Ansätzen aus der Zeit nach der Wende zum 20. Jahrhundert darstellen. Diese sind auch für die heutigen Analysen zum Teil relevant. Beispielsweise ist in der neueren sozialepidemiologischen Forschungsliteratur der Hinweis auf politischen Handlungsbedarf Teil des Genres. Ähnlich beinhaltet auch die soziale Hygiene nach Grotjahn nicht nur ärztliche, sondern auch politische Maßnahmen. Denn es gehe darum, alle (sozialen) Umwelteinflüsse zu untersuchen, die die Gesundheit des Volkskörpers beeinträchtigen könnten (Vgl. Grotjahn 1977 [1923], S. 5-6). Dabei sei nach Ansicht Grotjahns der Sozialismus eine Art Heilsbringer, der »die während der kapitalistischen Epoche errungene Gesundheitstechnik zum Gemeingut aller werden« (Grotjahn 1977 [1923], S. 6) lasse.

Ähnlich erklären bereits zehn Jahre zuvor Max Mosse und Gustav Tugendreich, dass Krankheiten sowohl von biologischen, als auch von sozialen Faktoren beeinflusst würden. Ihr Fokus liegt dabei auf den sozialen Gründen. Die »Ursache von Entstehung und Verlauf der Krankheiten [sei] ebensowenig etwas Einheitliches [...], wie der Begriff >Soziale Lage« (Mosse und Tugendreich 1977 [1913], S. 3). Es handele sich um komplexe Zusammenhänge, bei denen alle Einflüsse in den Blick genommen werden müssten, die das

10 | Analysiert werden im Folgenden die Reprints der beiden Standardwerke aus den siebziger Jahren, und damit jene Ausgaben, die den Sozialepidemiologien für ihre Literaturstände aller Wahrscheinlichkeit nach zur Verfügung standen. 
Gesellschaftsleben und die Kultur erzeugt habe. Gewöhnlich seien allerdings »beide Gruppen, soziale und natürliche (biologische), gemeinsam am Werke.« (Mosse und Tugendreich 1977[1913], S. 3).

Die Autoren beider Veröffentlichungen interessieren sich folglich nicht für den Einzelfall. Ihrer Meinung nach müsse die Aufmerksamkeit auf die (medizinische) Statistik gelenkt werden. Sie sei sowohl »Grundlage jeder sozialen Pathologie und jeder sozialen Hygiene« (Grotjahn 1977 [1923], S. IV), als auch die zentrale methodische Herangehensweise, um »die Einwirkung der sozialen Lage auf Entstehung und Verlauf von Krankheiten zu studieren « (Mosse und Tugendreich 1977[1913], S. 6). In der Analyse statistischer Daten $\mathrm{zu}$ Gesundheit und Krankheit findet sich so eine weitere Analogie $\mathrm{zu}$ den moderneren sozialepidemiologischen Studien. Letztere haben ebenfalls in der Erhebung quantitativer Daten und in der Auswertung von Variablen zu sozialen Konstrukten sowie von Variablen zur Morbidität und Mortalität der Untersuchungskohorte ein wesentliches Kennzeichen.

\subsection{Zur An- und Aberkennung des Rechts auf individuelle Lebensentwürfe bei Grotjahn}

Wirft man einen Blick auf die Arbeit Alfred Grotjahns, dann fallen im Kontext der Anerkennung von Gesundheit und Krankheit vor allem die Legitimationsstrategien auf, mit denen beispielsweise Forderungen von Zwangsmaßnahmen gegen bestimmte Personengruppen gerechtfertigt werden. Im Vergleich zu Mosse und Tugendreich erscheint er dabei als der radikalere Autor. Ihm geht es nicht nur um die Rechte, sondern auch um die »Pflichten des einzelnen dem Volksganzen gegenüber« (Grotjahn 1977 [1923], S. 7). Die in seiner Lesart zu Anfang des 20. Jahrhunderts propagierte soziale Hygiene setze als ihr »Endziel nichts mehr und nichts weniger als die ewige Jugend der eigenen Nation« (Grotjahn 1977 [1923], S. 9). Es seien daher

"Maßnahmen abzuleiten, die einem Volke, das in Sitte, Gesetz und Verwaltung diesen Regeln folgt, mit Sicherheit ermöglichen, die körperliche 
Grundlage seiner Kultur, seine Volkskraft, dauernd unversehrt zu erhalten “ (Grotjahn 1977 [1923], S. 9).

Alfred Grotjahn macht deutlich, dass es ihm nicht um Wohltätigkeit ginge, wenn er das Wort ssozial gebrauche, sondern um die Betonung des Gemeinschaftlichen (vgl. Grotjahn 1977 [1923], S. 10-11).

In seiner Auffassung stellt zudem Rasse einen wichtigen Einflussfaktor für das dar, was er mit >Sozialpathologien< bezeichnet. Zeige doch beispielsweise die wissenschaftliche Beschäftigung mit der Entstehung des Alkoholismus:

„im weitesten Sinne, daß einerseits Zustände des inneren Menschen, wie psychopathische Konstitution oder epileptische Anlage, und andererseits Zustände der Außenwelt, wie Klima, Rassenzugehörigkeit, Gestaltung des geselligen und öffentlichen Lebens, Form der Spirituosenproduktion und soziale Umwelt in ganz verschiedener Weise als Komponenten wirken können, um als Resultante dann den Alkoholmißbrauch als Massenerscheinung zu erzielen“ (Grotjahn 1977 [1923], S. 17).

Wie bereits einleitend erklärt, war Grotjahns Hauptanliegen eine von ihm als >gut < verstandene Bevölkerungspolitik auf wissenschaftlichen Grundlagen zu entwerfen. Insofern seine Sorge dem Geburtenrückgang galt, setzte er sich als sozialdemokratischer Reichstagsabgeordneter im Bereich des $₫ 218$ gegen Abtreibungen ein und außerdem gegen den Einsatz von Verhütungsmitteln und -methoden. Wichtig war ihm außerdem eine Verbesserung des Genbestands. Er teilte die Bevölkerung in die »zur Fortpflanzung völlig geeigneten, >die bedingt geeigneten< sowie die >Belasteten« (Kaspari 1989b, S. 218). Während die Geeigneten zur Fortpflanzung verpflichtet werden müssten, sollten die Belasteten von dieser ausgeschlossen werden.

Diese Einteilung in >Belastete $<$ und >Geeignete $<$ bildet den Kern von Grotjahns Anerkennungsansatz von gesunden bzw. kranken Menschen. Aus dieser Einteilung zog er normative Schlüsse für die Zu- bzw. Aberkennung von Rechten und Ansprüchen: Wer in diesem Raster an die falsche Stelle geriet, dem solle die Fortpflan- 
zung verboten oder per Zwangsmaßname unmöglich gemacht werden. An anderer Stelle erklärte Grotjahn, der Staat müsse, je nach Schwere des Falls, zwischen Asylierung, Verordnung >leichter< Präventivmittel, Schwangerschaftsabbruch oder Sterilisierung wählen (vgl. Kaspari 1989a, S. 310-311). In dem dialektischen Gerüst sozialer Wertschätzung und Missachtung unterscheidet der Sozialhygieniker aber ferner zwischen >Depravation< auf der einen Seite und >Degeneration< auf der anderen. Damit räumt er, wiederum ähnlich wie Max Mosse und Gustav Tugendreich, sozialen Umständen einen wichtigen Einfluss auf die Gesundheit ein. Als Depravation sei eine erworbene »körperliche Minderwertigkeit« (Grotjahn 1977 [1923], S. 17) aufgrund von Umwelteinflüssen zu bezeichnen, welche zwar zum Beispiel wegen Zuchthaushaft die >Rüstigkeit< vermindern und so zum Siechtum führen könne, welche sich aber nicht auf eine schlechte Erbanlage zurückführen lasse. Für die Gemeinschaft gefährlich hingegen sei die >Degeneration<. Denn, wenn:

"jemand unter den hygienisch und erzieherisch denkbar besten Bedingungen aufwächst und trotzdem ein Schwächling bleibt, so handelt es sich um eine ererbte konstitutionelle Minderwertigkeit, die man am besten als 'Degeneration ، von der oben erwähnten Depravation unterscheidet" (Grotjahn 1977 [1923], S. 17-18).

Somit fallen vor allem Wohlhabende und Reiche in seinen Fokus. >Degenerierte< würden:

"durch ungünstige Verhältnisse der Außenwelt schnell dahingerafft werden, während eine günstige Umwelt die Schwächlinge sorgfältig erhält und sie womöglich sich noch fortpflanzen läßt» (Grotjahn 1977 [1923], S. 18).

Implizit beinhaltet das sozialhygienische Konzept somit ein Verständnis von sozialer Auswahl, wobei die größte Gefahr für die Bevölkerung möglicherweise eher bei den Bessergestellten, als bei den Armen zu vermuten wäre, die wegen ihrer schwierigen sozialen Lage ohnehin schon stärker gesiebt würden, so der Denkansatz 
Grotjahns. Es ist zu betonen, dass Alfred Grotjahn sich stark vom Sozialdarwinismus, genauso wie vom Antisemitismus oder von arischem Gedankengut distanzierte. Er räumte zwar dem Darwinismus in der Auslegung etwa von Schallmayer oder Ploetz ein gewisses wissenschaftliches Recht ein. Dennoch hielt er diesen für eine deduktive Theorie, die eine induktive Einteilung in sungeeignet< und >rüstig « nicht ersetzen könne (vgl. Grotjahn 1977 [1923], S. 469470; siehe auch: Kaspari 1989a, S. 307 und S. 316-319).

Denn auf Grundlage von Degeneration müsse Entartung befürchtet werden, die sich als »körperliche oder geistige Verschlechterung der Nachkommen im Vergleich zu dem als vollkommen oder doch wenigstens nach dem Durchschnitt gemessen als im wesentlich fehlerfrei vorgestellten Vorfahren « (Grotjahn 1977 [1923], S. 468) verstehen lasse. Entsprechend müsse man im Sinne einer $>$ Fortpflanzungshygiene « die Minderwertigen durch die Maßnahmen der Geburtenprävention an der Erzeugung von unerwünschten Nachkommen« (Grotjahn 1977 [1923], S. 477) hindern.

Doch auch trotz der Unterscheidung zwischen Depravation und Degeneration führt Grotjahn eine lange Liste von Personen, denen er aus >fortpflanzungshygienischen $<$ Gründen die Zeugung von Nachkommen verwehren will.

„Folgende Gruppen benannte (in der Originalwortwahl) Grotjahn als für die Fortpflanzung gänzlich ungeeignet: Astheniker, Schwachsinnige, Epileptiker, Geisteskranke, an M. Basedow Leidende, Kranke mit Chorea Huntington, Trunksüchtige, sexuell Abartige, Taubstumme, erblich Augenkranke, Zuckerkranke, Konduktorinnen der Bluterkrankheit, chronisch Nierenkranke. Eine spätere, eugenisch interessiertere Zeit solle ihr Augenmerk auch auf die Träger ,verdeckter Anlagen r richten und nur noch die 'Vollrüstigen، zur Fortpflanzung zulassen." (Kaspari 1989a, S. 311)

Aufforderungen zum Ausschluss aus der Fortpflanzungsgemeinschaft finden sich nicht nur in anderen Arbeiten, sondern auch in dem von der heutigen Literatur immer wieder zitierten Werk. So gelte etwa für die Basedowsche Krankheit, dass die Kranken 
"[w]ie bei den meisten erblich begründeten Krankheitszuständen, [...] imstande [seien], entweder die Erkrankung selbst oder doch wenigstens eine psychische Minderwertigkeit ihren Nachkommen weiterzuvererben. Die mit Basedowscher Krankheit, auch in ihrer leichtesten Form, behafteten Frauen sind also für die Fortpflanzung unerwünscht und blieben besser davon ausgeschlossen“ (Grotjahn 1977 [1923], S. 310).

In dem szientistischen Gerüst von Alfred Grotjahn wird somit nach Überlegungen, welche bereits nach der Machtübertragung an die Nationalsozialisten datieren, etwa einem Drittel der Bevölkerung (vgl. Rüdin 1934, S. 15) Anerkennung mit Bezug auf wissenschaftliche Begründungsstrategien vorenthalten. Die sozialhygienisch begründete Missachtung von Menschen eskaliert bis hin zur Forderung von Zwangsmaßnahmen gegenüber Kranken. >Degenerierte< Individuen, die durch ihre Fortpflanzung die >Volksgemeinschaft entarten< könnten, müssten mit den Mitteln der Geburtenprävention kontrolliert werden. Zwar lehnte er Euthanasie scharf ab, sprach sich aber dennoch dafür aus, dass der Gesetzgeber Zwangssterilisierungen einführen dürfe (vgl. Kaspari 1989b, S. 221). Die Konzentrierung auf den Volkskörper kann dabei als ein Indiz für eine am aggregierten Kollektivwohlergehen (unter bewusstem Ausschluss bzw. Diskriminierung bestimmter Bevölkerungsgruppen) orientierte zugrundeliegende Vorstellung von Gerechtigkeit gewertet werden. Von dieser lassen sich Ansprüche auf Gesundheitsleistungen, aber auch staatliche Rechte $\mathrm{zu}$ medizinischen Interventionen am Einzelnen (wie etwa Zwangssterilisationen) ableiten. Die Konzentrierung auf den Volkskörper ging einher mit einer stark pointierten Missachtung jener Kranken, die im wissenschaftlichen Raster der Sozialhygiene als degeneriert klassifiziert wurden. In der Arbeit Alfred Grotjahns zeichnet sich damit eine starke Betonung von Normen ab, die sich am Kollektiv orientieren bei gleichzeitiger Missachtung der Rechte des Individuums. 


\subsection{Soziale Lage und gesellschaftliche (Miss-)Achtung im Werk von Mosse und Tugendreich}

Während sich bei Alfred Grotjahn Legitimationsstrategien für Zwangsmaßnahmen finden lassen, steht in der folgenden Betrachtung des Werkes von Mosse und Tugendreich deren Definition von >sozialer Fürsorge< im Vordergrund, sowie deren wissenschaftliches Verhältnis zu Alfred Grotjahn. Wie bereits erwähnt, erklären auch Max Mosse und Gustav Tugendreich, dass sowohl die soziale Lage als auch kulturelle Faktoren neben der natürlich-biologischen Seite die Gesundheit beeinflussten. Sie bedienen sich ebenso wie Grotjahn statistischer Datensätze für ihre Analysen. Genauso wie dieser distanzieren sie sich stark von sozialdarwinistischem Gedankengut. Ihrer Ansicht nach verfälsche soziale Fürsorge nicht einen natürlichen Ausleseprozess; überhaupt seien hier unterschiedliche Kräfte am Werk. Es müsse

"mit aller Deutlichkeit gesagt werden, dass die durch die soziale Lage herbeigeführte Siebung in keiner Hinsicht gleichzusetzen ist der auslesenden Wirkung der natürlichen Einflüsse" (Mosse und Tugendreich 1977[1913], S. 19).

Nach Mosse und Tugendreich dürfe man daher nicht davon ausgehen, dass soziale Fürsorge zu einer »Dekadenz des Volkes und der Rasse« (Mosse und Tugendreich 1977[1913], S. 19) führe.

Im Vergleich zu Grotjahn müssen aber auch die Unterschiede zu Mosse und Tugendreich (Seidler 2008, S. 26) betont werden. Grotjahns Blick ist auf das »generative Konglomerat« (Kaspari 1989a, S. 316; vgl. auch Weindling 1984) gerichtet. Ihm geht es damit um die Vererbung von genetischen Bestimmungsfaktoren auf die nächste Generation. Dabei betont er immer wieder die allgemeingültige Wertfreiheit seiner Theorie. Im Prinzip ließen sich seine Vorschläge auf jegliche Gruppierung von Menschen anwenden. Mosse und Tugendreich hingegen legen den Fokus der Arbeit in 
dem von ihnen herausgegebenen Sammelwerk klar auf soziale Einflüsse. In der Sprache Grotjahns würden sie sich ausschließlich mit der >Depravation< beschäftigen. Während Mosse und Tugendreich erklären, aus der Statistik ${ }^{11}$ gehe ohne weiteres hervor, dass »beispielsweise bei der Tuberkulose, die soziale Lage im höchsten Masse infektionsbefördernd einwirkt (Mosse und Tugendreich 1977[1913], S. 4), sieht auch Grotjahn den sozialen Einfluss auf die Tuberkulose, möchte aber dennoch Tuberkuloseverdächtige von Heirat und Fortpflanzung ausschließen (vgl. Grotjahn 1926, S. 189).

Faktoren wie Klima und Rasse sind jedoch auch für Mosse und Tugendreich eindeutig Einflussfaktoren auf die Gesundheit, die den Einfluss der sozialen Lage verschleiern oder verdecken könnten (Mosse und Tugendreich 1977[1913], S. 4-5). Die Annahme, dass »Rasse und Klima überhaupt Einfluss haben, [könne, Deklination in den Konjunktiv durch SK] allerdings kaum bestritten werden.« (Mosse und Tugendreich 1977[1913], S. 5). Als Beweis setzen sich die beiden Autoren mit Studien >wohlverstandener Rassentheorie< (vgl. Mosse und Tugendreich 1977[1913], S. 5) auseinander. So zitieren sie beispielsweise Sofers Studie über die jüdische Widerstandskraft gegen Tuberkulose. In New York, London und Chicago hätten die

m'Juden trotz der ungünstigen hygienischen Verhältnisse und des Schwitzsystems doch eine erhöhte Widerstandskraft gegen Tuberkulose “ (Sofer, zitiert nach: Mosse und Tugendreich 1977[1913], S. 5)

gezeigt. ${ }^{12}$ Dabei möchten sie allerdings auch diese rassischen Einflüsse zum Teil durch ihr Konzept der sozialen Lage einhegen. Es dürfe

$1 \mathbf{1}$ | Leider erklären die beiden Autoren an dieser Stelle nicht, auf welche Statistik sie sich beziehen. Relevant erscheint mir hier auch weniger der geführte Nachweis der Behauptung, als vielmehr die allgemeingültige Wahrheit beanspruchende Rhetorik.

12 | Siehe für den Originaltext von Sofers Studie: Sofer 1910. 
"nicht übersehen werden, dass bestimmte Klimata und auch Rassen oft bestimmte Berufe oder bestimmte Lebensweisen begünstigen, so dass dann die Eigentümlichkeiten der Morbidität und Mortalität unter diesem Klima, bei dieser Rasse nicht diesen selbst zuzuschreiben sind, sondern dem durch sie bedingten sozialen Milieu." (Mosse und Tugendreich 1977[1913], S. 5)

Dennoch wird auch in dieser Einschränkung deutlich, dass für Mosse und Tugendreich die >Rasse< eine wissenschaftliche Kategorie ist, deren Einfluss auf Gesundheit und Krankheit durchaus anerkannt werden müsse. Dies wird auch klar, wenn sie im Anschluss an ein langes Zitat aus einem frühen Werk von Alfred Grotjahn, der sich bereits 1904 von sozialdarwinistischer Rassentheorie distanziert, von »Bestrebungen vernünftiger Vererbungshygieniker« (Mosse und Tugendreich 1977[1913], S. 21) sprechen. ${ }^{13}$ Solche Vererbungshygieniker, zu denen ihrer Meinung nach Alfred Grotjahn zu zählen ist, zielten

"nicht auf Verschärfung der Vitalauslese, sondern nur auf Verschärfung und Vervollkommnung der Fortpflanzungsauslese. Die Sozialhygiene soll also aus rassebiologischen Gründen nicht eingeschränkt, sondern ergänzt werden durch Massnahmen, welche die Fortpflanzung minderwertiger Eigenschaften verhüten." (Mosse und Tugendreich 1977[1913], S. 21)

Während sich Mosse und Tugendreich ebenso wie Grotjahn gegen Euthanasie aussprechen, äußern sie sich hier eindeutig bejahend zu dessen Vorstellungen über Geburtenplanung. Sie nehmen letzten Endes Zwangsmaßnahmen bis hin zur Sterilisierung von Menschen mit >minderwertigen< Erbanlagen in Kauf.

Christoph Kaspari spricht von einem eugenischen Konzept, welches bereits vor 1933 in der Medizin und Hygiene entstanden sei. An diesem sieht er Alfred Grotjahn beteiligt (vgl. Kaspari 1989a, S. 325). Eine ähnliche Auffassung vertritt Paul Weindling, wenn er erklärt, dass die Eugenik sich bereits vor 1933 entwickelt habe, um

13 | Siehe für das von Mosse und Tugendreich zitierte Werk: Grotjahn 1904. 
dann ein wesentlicher Bestandteil der sozialen Medizin zu werden (vgl. Weindling 1985). Beide Werke stellen damit Teil einer Entwicklung hin zum nationalsozialistischen Präventionsverständnis dar, das »ideologisch verbrämt Krankheit weitgehend negierte (Madarász 2010, S. 162).

Die soziale Lage ist für Mosse und Tugendreich ein Kernbestandteil des Zusammenhangs unterschiedlicher Häufigkeiten von Gesundheit und Krankheit in der Gesellschaft. Sie verteidigen ihre Idee sozialer Fürsorge, die nicht aufgrund eines falsch verstandenen Sozialdarwinismus verhindert werden dürfe. Doch auch wenn Mosse und Tugendreich selbst die soziale Lage in den Mittelpunkt stellen, schließen sie eugenische Überlegungen zur Geburtenprävention nicht explizit aus. Im Gegenteil erklären sie unter anderem den Faktor >Rasse $<$ zum wichtigen Einflussfaktor, selbst wenn für sie die konkreten Ausformungen von Gesundheit und Krankheit jeweils immer auch über die soziale Lage gedeutet werden müsse. Mit der klaren Positionierung für den sozialdemokratischen Sozialhygieniker aus Berlin zeigen sie sich erheblich von dem eugenischen Konzept im Sinne Kasparis beeinflusst. Allerdings verweigern sie selbst Kranken, die aufgrund ihrer Erbanlage erkrankt sind, nicht die Anerkennung als vollständige Person in einem Honnethschen Sinne. Dass jedoch eine Einteilung in >belastete $<$ und für die >Fortpflanzung geeignete< Bevölkerungsteile durchaus Sinn machen könnte, scheint den beiden Autoren einzuleuchten. Implizit kommt so auch bei ihnen eine Norm der Maximierung des Kollektivnutzens zum Tragen, die Menschen mit Anlagen für genetische Erkrankungen zugunsten eines >Gesamtwohls < missachtet; die Rechte der Individuen auf selbstbestimmte Lebensvollzüge treten bisweilen hinter die Rechte und Interessen des Kollektivs zurück. 


\section{Von der Optimierung des Kollektivs ZUR FOKUSSIERUNG AUF DIE INDIVIDUELLE GESUNDHEIT? EXEMPLARISCHE ÜBERLEGUNGEN Zum Bedeutungswandel des Rechts auf GESUNDHEITLICHE LEISTUNGEN IN historischer Perspektive}

Während die soziale Hygiene in Anlehnung an Grotjahn aus heutiger Sicht stark nationalistisch aufgeheizt erscheint, wie Grotjahn schrieb, zielte sie auf die »ewige Jugend der eigenen Nation « (Grotjahn 1977 [1923], S. 9), kann meines Erachtens nicht von einem >völkischen< Erkenntnisinteresse der sozialepidemiologischen Forschung gesprochen werden. Besonders nach dem Zweiten Weltkrieg entwickelte sich der Trend, in vergleichenden Statistiken unterschiedliche Variablen als soziale Einflüsse auszuwerten. Die Vielfalt der untersuchten sozialen Faktoren hat zur Einführung der Unterscheidung von shorizontaler< sozialer Ungleichheit auf der einen Seite geführt, wie zum Beispiel Alter, Geschlecht oder Nationalität. Auf der anderen Seite wird das Spektrum zwischen Armut und Reichtum in Form von Variablen wie etwa Einkommen, Gehalt oder Vermögen als svertikale< soziale Ungleichheit erhoben (vgl. exemplarisch Lampert et al. 2002). Eine Fokussierung auf den >Volkskörper< in diesem Rahmen erfolgt nicht mehr. Damit verliert auch gleichzeitig die Norm des Volkskörpers als Kollektiv, dessen Nutzen (im Hinblick auf die nächstfolgenden Generationen) es im Sinne eines aggregierten Gesamtnutzens zu maximieren gilt, an Bedeutung. Dadurch wird Raum eröffnet, um beispielsweise die Perspektive auf individuelle Ansprüche und Lebensentwürfe aufnehmen zu können.

In den Forschungsdesgins der hier zugrundeliegenden Publikationen finden sich induktive Hinweise auf beide Verständnisse wieder. Darüber hinaus gibt es eine Reihe von Publikationen, die sich nicht einer der beiden dichotomen Ausprägungen zuordnen lassen, sondern die eine hybride Auffassung vertreten, in welcher sich sowohl Elemente individueller als auch kollektiver Argumentationen zeigen. 
Schaefer führt beispielsweise 1996 unterschiedliche Sterblichkeiten in verschiedenen sozioökonomischen Schichten $\mathrm{zu}$ einem bedeutenden Teil auf verschiedene Lebensstile zurück. Diese können entweder ein höheres oder ein geringeres Gesundheitsrisiko mit sich bringen. Schichtspezifische Ungleichheiten der Gesundheit seien »zu einem guten Teil von den Risikofaktoren des Verhaltens « (Schaefer 1996, S. 445) abhängig. Schaefer lässt sich daher in eine Logik gesundheitsrelevanten Verhaltens einordnen. ${ }^{14}$ Für diese lässt sich die Frage stellen, ob bzw. inwiefern dem Individuum heute ein höherer Wert anerkannt wird als in den älteren Werken. Man kann argumentieren, dass hier die Gesundheit der gesamten Bevölkerung nur noch vermittelt über die Gesundheit des Individuums verbessert werden soll. In dieser Richtung sozialepidemiologischer Forschung verursachen somit nicht mehr gesellschaftliche Verhältnisse Krankheiten. Vielmehr tritt an die Stelle von Ursachenüberlegungen im Kontext der Vererbungshygiene die Verantwortung des Einzelnen für seine Gesundheit. Spitzt man die Grundannahmen $\mathrm{zu}$, dann soll sich hier das Individuum gegenüber seinem zukünftigen Selbst >fair< verhalten. Das tue es, indem es eine bewusste gesundheitsrelevante Verhaltensweise an den Tag lege und so lebe, dass es auch in der Zukunft gesund bleibe. Der Einzelne, so die Interpretation, ist entsprechend primär für sich selbst und seine Gesundheit verantwortlich.

Des Weiteren gibt es Autoren, die kollektive Pflichten und Bedürfnisse stärker achten. So werden beispielsweise die öffentlichen Gesundheitsdienste in die Verantwortung genommen, um gemeinsam mit der Sozialepidemiologie sozio-ökonomische Unterschiede in der Gesundheit zu reduzieren (vgl. Gardemann 2001). Auch Brand und Schmacke lassen sich meines Erachtens nach der Achtung von Normen, welche sich am Kollektiv orientieren zuordnen (Brand und Schmacke 1998). Sie erklären, dass der öf-

14 | Vergleiche exemplarisch: Karmaus 1981; Blane, Bartley, und Smith, 1997; Gostomzyk und Schaefer 1998; Steinkamp 1999; Richter und Mielck 2000; Swart 2001; Rosenbrock und Kümpers 2006. 
fentliche Gesundheitsdienst die Chancenungleichheit für Gesundheit immer wieder zum Thema machen müsse. Sie beziehen sich dabei ausdrücklich auf die Thesen Wilkinsons, nach dem soziale Benachteiligungen mit gesundheitlichen Ungleichheiten einhergingen. In seinem, für das Fach zentralen Artikel, bezieht Richard Wilkinson die nationale Mortalitätsrate auf die Weite der Armutsschere (Wilkinson 1992). Konsequenterweise lässt sich eine geringere Mortalitätsrate bei einer gleicheren Einkommensverteilung erwarten. Eine bessere Gesundheit der Gruppe ließe sich demnach durch eine gemeinschaftliche Umverteilung und nicht durch die individuelle Verantwortung zum gesundheitsrelevanten Verhalten erreichen. Während dieser Argumentation ebenfalls der Gedanke einer Maximierung eines Kollektivwohls zugrundeliegt, zeigt sich jedoch im Gegensatz zu einer Rechtfertigung über den kollektiven Volkskörper keine Missachtung einzelner Kranker zugunsten des Volkskörpers als Ganzem. Vielmehr wird das Individuum durch eine Verbesserung der individuellen Position in der Gesellschaft gesund erhalten.

Eins der wichtigsten Mittel zur Angleichung der Morbiditäten in der Gesellschaft läge nach der Ansicht von Autoren, die sich diesem kollektivistischen Verständnis zuordnen lassen, an der den Betroffenen gegenüber ausgesprochenen Anerkennung. Ungleichheiten aufgrund sozialer Benachteiligung würden durch emotionale Unsicherheiten in der frühkindlichen Entwicklung verschlimmert.

"Es sei insofern von größter Bedeutung, Menschen glaubhaft das Gefühl zu vermitteln, daß sie wertgeschätzt werden und zur Gesellschaft zugehörig betrachtet werden" (Brand und Schmacke 1998, S. 630).

Somit läge bei ihnen die Lösung sozialer Ungleichheiten nicht in der Missachtung kranker Individuen. Publikationen, die sich diesem neueren kollektiven Normverständnis zuschreiben lassen, brächten vielmehr über gemeinschaftliche Maßnahmen einzelnen Individuen ihre Achtung zum Ausdruck. 
Schließlich lassen sich einige Ansätze als Vertreter hybrider Verständnisse deuten, in denen sich sowohl Merkmale für die Achtung des Individuums, als auch des Kollektivs finden lassen (vgl. Gostomzyk 2001, S. 226-227; Trojan 2001 S. 44; Rosenbrock und Kümpers 2006). Prägnant schlagen beispielsweise Elkeles und Mielck ein systematisiertes Modell zur Erklärung gesundheitlicher Ungleichheiten vor (Elkeles und Mielck 1997). Soziale Ungleichheit präge eine Reihe von Gesundheitsrisiken, die auf der Ebene des Individuums anzusiedeln sind, wie dem Risikoverhalten. Sozial ungleich verteilte Gesundheitsrisiken finden sich aber auch auf der Ebene gemeinschaftlicher Güter, in etwa unterschiedliche gesundheitliche Belastungen bei der Arbeit oder in der Wohnungsumgebung, divergierende Ressourcen zur Bewältigung von Krankheitsrisiken oder Unterschiede in der gesundheitlichen Versorgung (vgl. Elkeles und Mielck 1997, S. 140). Kollektive Einflüsse auf die Gesundheit führen in diesem Modell aber zu Veränderungen im gemeinschaftlichen Rahmen, etwa einer Verbesserung der Versorgungslage und nicht zu individuellen Konsequenzen, wie dies bei Alfred Grotjahn der Fall war. Die Missachtung individueller Rechte aus dem ersten Viertel des 20. Jahrhunderts findet sich somit in dieser neueren $\mathrm{Pu}$ blikation nicht wieder. Im Gegensatz stehen sie für die Anerkennung des individuellen Rechts auf Gesundheit durch beispielsweise eine bessere Verteilung im Gesundheitswesen.

\section{FAZIT UND WEITERFÜhRENDE ÜBERLEGUNGEN ZUR ANALYSE DER ANERKENNUNG VON KRANKHEIT UND GeSUNDhEIT IM HISTORISCHEN VERGLEICH}

Sowohl Max Mosse und Gustav Tugendreich als auch Alfred Grotjahn grenzen sich stark von sozialdarwinistischen und antisemitischen Strömungen und Überlegungen ab. In beiden Werken wird Patientenmord und Euthanasie abgelehnt, in beiden Büchern finden sich aber auch eine mehr oder weniger starke Billigung von staatlichen Zwangsmaßnahmen gegenüber Kranken, im Sinne der von 
den Autoren vertretenen Bevölkerungspolitik. Alfred Grotjahn kategorisiert die von ihm untersuchten Populationen als >geeignet< oder sungeeignet für die Fortpflanzung. Er spricht klare Empfehlungen aus, wer von der Fortpflanzung besser ausgeschlossen bliebe und fordert, dass bestimmte Patientinnen zur Not sterilisiert werden müssten, um einer >Entartung des Volkskörpers< vorzubeugen. Als gefährlich für die Gemeinschaft sieht er vor allem jene schwachen und kranken Menschen, die sozial unter besten Umständen aufwachsen würden, weil ihre Mängel nicht auf ein erworbenes, sondern auf ein ererbtes Defizit zurückzuführen seien.

Demgegenüber versuchen Max Mosse und Gustav Tugendreich, den Einfluss von Krankheit und Gesundheit mit der sozialen Lage zu erklären. Auch den Einfluss von Klima und Rasse möchten sie als einen über die soziale Lage vermittelten Einfluss verstehen. Dennoch erklären sie sich einverstanden mit den Forderungen »vernünftiger Vererbungshygieniker« (Mosse und Tugendreich 1977[1913], S. 21), zu denen sie Alfred Grotjahn offensichtlich zählen.

In unterschiedlichem Ausmaße erkennen die Autoren der beiden Publikationen so nur bestimmte Teile der Bevölkerung vollständig an. Anderen Bevölkerungsgruppen wird das individuelle Recht bzw. der Anspruch auf Fortpflanzung abgesprochen und zudem Zwangsmaßnahmen wie beispielsweise Zwangssterilisationen befürwortet. Die Autoren fordern sogar, dass solchen Personengruppen die Ehe (und eigene Kinder) zu verbieten sei. Einen Wert für den >Volkskörper< haben letzten Endes nur jene, die als >rüstig< eingeschätzt werden. Sie schließen damit große Teile der Bevölkerung von der öffentlichen Partizipation in Politik und Gesellschaft aus, aber auch von individuellen Lebensentwürfen (wie etwa Familiengründung). Hier herrschte eine Fokussierung auf das Kollektiv vor, nach der sich spezifische Einzelinteressen dem Gesamtwohl unterzuordnen hatten und keine Berücksichtigung mehr fanden. Diese allein auf das Kollektivwohl ausgerichtete Sichtweise erscheint aus der Perspektive der sozialepidemiologischen Literatur als eine eklatante Missachtung bzw. Diskriminierung von Menschen mit z.B. Anlagen für Erbkrankheiten. 
Vergleicht man diese Formen der Achtung von Krank und Gesund mit jenen in der sozialepidemiologischen Literatur seit den 196oer Jahren, zeigen sich Veränderungen in der impliziten Achtung der Rechte, Bedürfnisse und Pflichten des Individuums sowie des Kollektivs. Während Anfang des 20. Jahrhunderts das Recht auf Gesundheit eingeschränkt werden konnte, scheint dies heute in dieser Form nicht mehr möglich zu sein. Die Veränderung liegt zum Teil im Wandel des Adressaten, gegenüber welchem man sich gerecht verhalten solle. Es steht nicht mehr der kollektive Volkskörper im Mittelpunkt.

In neuerer Forschung tritt dem Gedanken einer solchen einzig am Kollektivwohl orientierten Position zumindest die Achtung dem kranken Individuum gegenüber hinzu. Darüber hinaus finden sich Hinweise auf eine stärkere Anerkennung der Rechte und Ansprüche dem zukünftigen Individuum gegenüber. In Arbeiten mit dem Fokus auf das gesundheitsrelevante Verhalten wird paradigmatisch die individuelle Verantwortung für die eigene Gesundheit in den Vordergrund gerückt. Schließlich zeigen sich auch hybride Formen, in denen Argumentationen, die sich am aggregierten Gesamtwohl einer Bevölkerung orientieren, mit solchen ergänzt werden, die für eine stärkere Berücksichtigung der Perspektive und Ansprüche der Individuen sprechen. In den hier analysierten sozialepidemiologischen Publikationen wurde also trotz des Interesses an für die Untersuchungskohorte allgemeingültigen Erkenntnissen die Stellung des Individuums deutlich gestärkt.

Wie bereits oben ausgeführt, baut das hier vorgeschlagene Konzept der Unterscheidung zwischen >Maximierung des Kollektivwohls< und >Maximierung des Wohl des Individuums< auf einer induktiven Analyse eines begrenzten Teils eines größeren Textkorpus auf. Zur weiterführenden Arbeit müsste die Analyse um eine Diskussion der Arbeiten von anderen Sozialhygienikern wie Adolf Gottstein (1857-1941) und um die Schriften von Rassenhygieniker, von denen sich Grotjahn distanziert hatte, wie Friedrich Wilhelm Schallmayer (1857-1919), Alfred Ploetz (1860-1940), Ernst Rüdin (1874-1952) ergänzt werden. Dennoch lässt sich bereits festhalten, 
dass der heutigen Sozialepidemiologie neben dem Verweis auf die frühe Auswertung von Statistiken in Hinblick auf soziale Einflussfaktoren auf Krankheit bei Alfred Grotjahn, Max Mosse und Gustav Tugendreich ein weiterer Verweis offensteht. Nicht nur haben sich Autoren bereits zu Beginn des 20. Jahrhunderts mit der Korrelation von Armut und Krankheit befasst, heutige sozialepidemiologische Forschungen grenzen darüber hinaus nicht über einen konstruierten Szientismus ganze Bevölkerungsteile wegen ihrer Krankheit aus der Gesellschaft aus.

\section{LITERATUR}

Bardehle, D. (2001): Sozial-Epidemiologische Indikatoren in der Gesundheits- und Sozialstatistik. In: Mielck, A. und Bloomfield, K. (Hg.): Sozial-Epidemiologie. Eine Einführung in die Grundlagen, Ergebnisse und Umsetzungsmöglichkeiten. Weinheim/München: Juventa-Verlag, 341-357.

Brand, H.; Schmacke, N. (1998): Soziale Ungleichheit und Gesundheit: Die Rolle kommunaler Gesundheitsdienste in Deutschland. Das Gesundheitswesen 6o(11): 626-631.

Deppe, H.U. (1983): Vorsicht vor Alfred Grotjahn! Sozialhygiene und Eugenik. Demokratisches Gesundheitswesen (5): 23.

Elkeles, T.; Mielck, A. (1997): Entwicklung eines Modells zur Erklärung gesundheitlicher Ungleichheit. Das Gesundheitswesen 59(3): 137-143.

Fangerau, H. (2001): Etablierung eines rassenhygienischen Standardwerkes 1921-1941. Der Baur-Fischer-Lenz im Spiegel der zeitgenössischen Rezensionsliteratur. Frankfurt a.M.: Lang (u.d.T.: Fangerau, Heiner: Das Standardwerk zur menschlichen Erblichkeitslehre und Rassenhygiene von Erwin Baur, Eugen Fischer und Fritz Lenz im Spiegel der zeitgenössischen Rezensionsliteratur 1921 1941, Univ. Diss. Bochum 2000).

Fangerau, H. (2013): Armut, Arbeit, Menschenwert: Anerkennung und Selbstkonstitution als Schlüsselkategorien in der eugeni- 
schen Bewegung des frühen 20. Jahrhunderts. In: Fangerau, H. und Kessler, S. (Hg.): Achtung und Missachtung in der Medizin. Anerkennung und Selbstkonstitution als Schlüsselkategorien zur Deutung von Krankheit und Armut. Freiburg/München: Verlag Karl Alber, 69-97

Fraser, N.; Honneth, A.; Wolf, B.; (2003): Umverteilung oder Anerkennung? Eine politisch-philosophische Kontroverse. Orig.-Ausg., 1. Auflage. Frankfurt a.M.: Suhrkamp.

Gardemann, J. (2001): Kommunikation zwischen Sozial-Epidemiologie und dem öffentlichen Gesundheitsdienst (ÖGD). In: Mielck, A. und Bloomfield, K. (Hg.): Sozial-Epidemiologie. Eine Einführung in die Grundlagen, Ergebnisse und Umsetzungsmöglichkeiten. Weinheim/München: Juventa-Verlag, 277-285.

Gostomzyk, J.G. (2001): Sozialepidemiolgoie in der Sozialmedizin. In: Mielck, A. und Bloomfield, K. (Hg.): Sozial-Epidemiologie. Eine Einführung in die Grundlagen, Ergebnisse und Umsetzungsmöglichkeiten. Weinheim/München: Juventa-Verlag, 219-230.

Gostomzyk, J.G.; Schaefer, H. (1998): Gegenwart und Zukunft der Sozialmedizin. Das Gesundheitswesen 6o(1): 3-12.

Grotjahn, A. (1904): Soziale Hygiene und Entartungsproblem. In: Weyl, T. (Hg.): Handbuch der Hygiene. Soziale Hygiene. Vierter Supplement-Band. Jena: Verlag von Gustav Fischer, 727-790.

Grotjahn, A. (1926): Die Hygiene der menschlichen Fortpflanzung. Versuch einer praktischen Eugenik. Berlin/Wien: Urban \& Schwarzenberg.

Grotjahn, A. (1977 [1923]): Soziale Pathologie. Versuch einer Lehre von den sozialen Beziehungen der Krankheiten als Grundlage der sozialen Hygiene. Berlin: Springer.

Habermas, J. (1991): Moralbewusstsein und kommunikatives Handeln. 4. Auflage. Frankfurt a.M.: Suhrkamp.

Habermas, J. (1999): Die Einbeziehung des Anderen. Studien zur politischen Theorie. 1. Auflage. Frankfurt a.M.: Suhrkamp.

Habermas, J. (2001): Erkenntnis und Interesse. Mit einem neuen Nachwort. 13. Auflage. Frankfurt a.M.: Suhrkamp. 
Habermas, J. (2009): Theorie des kommunikativen Handelns. [Nachdr.]. Frankfurt a.M.: Suhrkamp (Band 1: Handlungsrationalität und gesellschaftliche Rationalisierung. Band 2: Zur Kritik der funktionalistischen Vernunft).

Heinrich-Weltzien, R.; Kühnisch, J.; Goddon, I.; Senkel, H.; Stößer, L. (2007): Zahngesundheit deutscher und türkischer Schüler Ein 10-Jahresvergleich. Das Gesundheitswesen 69(2): 105-109.

Honneth, A. (1990): Die zerrissene Welt des Sozialen. Sozialphilosophische Aufsätze. 1. Auflage. Frankfurt a.M.: Suhrkamp.

Honneth, A. (2000): Das Andere der Gerechtigkeit. Habermas und die ethische Herausforderung der Postmoderne. In: Honneth, A.: Das Andere der Gerechtigkeit. Aufsätze zur praktischen Philosophie. 1. Aufl., Orig.-Ausg. Frankfurt a.M.: Suhrkamp, 133-170.

Honneth, A. (2002): Kommunikatives Handeln. Beiträge zu Jürgen Habermas Theorie des kommunikativen Handelns, erw. u. aktualisierte Auflage. Frankfurt a.M.: Suhrkamp.

Honneth, A. (2008): Kampfum Anerkennung. Zur moralischen Grammatik sozialer Konflikte. 1. Auflage, [Nachdr.]. Frankfurt a.M.: Suhrkamp.

Karmaus, W. (1981): Präventive Strategien und Gesundheitsverhalten. Das Argument: Zeitschrift für Philosophie und Sozialwissenschaften (Sonderband, 64): 7-26.

Kaspari, C. (1989a): Der Eugeniker Alfred Grotjahn (1869-1931) und die »Münchner Rassenhygieniker«. Der Streit um »Rassenhygiene oder Eugenik?«. Medizinhistorisches Journal 24(3/4): 306332.

Kaspari, C. (1989b): Alfred Grotjahn (1869-1931). Leben und Werk. Inaugural-Dissertation zur Erlangung des Doktorgrades der Hohen Medizinischen Fakultät der Rheinischen-Friedrich-Wilhelms-Universität zu Bonn. Bonn: Dissertationsdruck M. Wehle.

Kolip, P. (2004): Der Einfluss von Geschlecht und sozialer Lage auf Ernährung und Übergewicht im Kindesalter. Bundesgesundheitsblatt - Gesundheitsforschung - Gesundheitsschutz 47(3): 235-239. 
Koppitz, U.; Labisch, A. (2007): Grotjahn, Alfred. In: Gerabek, W.; Haage, B.; Keil, G.; Wegner, W. (Hg.): Enzүklopädie Medizingeschichte. Berlin: Walter De Gruyter, 513.

Labisch, A. (1992): Homo hygienicus. Gesundheit und Medizin in der Neuzeit. Frankfurt a.M.: Campus.

Lampert, T.; Schenk, L.; Stolzenberg, H. (2002): Konzeptualisierung und Operationalisierung sozialer Ungleichheit im Kinder- und Jugendgesundheitssurvey. Das Gesundheitswesen 64(1): 48-52.

Lengwiler, M.; Madarász, J. (2010): Präventionsgeschichte als Kulturgeschichte der Gesundheitspolitik. In: Lengwiler, M.; Madarász, J. (Hg.): Das präventive Selbst. Eine Kulturgeschichte moderner Gesundheitspolitik. Bielefeld: transcript, 11-28.

Madarász, J. (2010): Die Pflicht zur Gesundheit. Chronische Krankheiten des Herzkreislaufssystems, zwischen Wissenschaft, Populärwissenschaft und Öffentlichkeit, 1918-1945. In: Lengwiler, M.; Madarász, J. (Hg.): Das präventive Selbst. Eine Kulturgeschichte moderner Gesundheitspolitik. Bielefeld: transcript, 137-167.

Mielck, A. (1994): >Soziale Medizin «. Die Diskussion zu Beginn des 20. Jahrhunderts. In: Mielck, A. (Hg.): Krankheit und soziale Ungleichheit. Opladen: Leske + Budrich, 35-52.

Mielck, A. (2000): Soziale Ungleichheit und Gesundheit. Empirische Ergebnisse, Erklärungsansätze, Interventionsmöglichkeiten. 1. Auflage. Bern/Göttingen/Toronto/Seattle: Huber.

Mildenberger, F. (2002): Auf der Spur des »scientific pursuit«. Franz Josef Kallmann (1897-1965) und die rassenhygienische Forschung. Medizinhistorisches Journal 37(2): 183-200.

Mosse, M.; Tugendreich, G. (1977[1913]): Krankheit und soziale Lage. München: Lehmanns.

Puska, P.; Keller, I. (2004): Primärprävention von nicht übertragbaren Krankheiten. Erfahrungen von bevölkerungsbasierten Interventionen in Finnland bis zur globalen Arbeit der WHO. Zeitschrift für Kardiologie 93(2): 37-42.

Richter, M.; Mielck, A. (2000): Strukturelle und verhaltensbezogene Determinanten gesundheitlicher Ungleichheit. Journal of Public Health 8(3): 198-215. 
Roelcke, V. (2002): Programm und Praxis der psychiatrischen Genetik an der Deutschen Forschungsanstalt für Psychiatrie unter Ernst Rüdin: Zum Verhältnis von Wissenschaft, Politik und Rasse-Begriff vor und nach 1933. Medizinhistorisches Journal 37: 21-55.

Rosenbrock, R.; Kümpers, S. (2006): Zur Entwicklung von Konzepten und Methoden der Prävention. Psychotherapeut 51(6): 412420.

Rüdin, E. (Hg.) (1934): Erblehre und Rassenhygiene im völkischen Staat. München: Lehmanns.

Satzinger, H. (2002): Krankheiten als Rassen. Politische und wissenschaftliche Dimensionen eines biomedizinischen Forschungsprogramms von Cécile und Oskar Vogt zwischen Tiflis und Berlin (1919-1939). Medizinhistorisches Journal 37(3-4): 301350.

Schaefer, H. (1996): Beruf und Krankheit. Das Gesundheitswesen 58(8-9): 442-446.

Seidler, E. (2008): » ... die glückliche Spezialität der Kinderheilkunde«. 125 Jahre deutsche Gesellschaft für Kinder- und Jugendmedizin. In: Historische Kommission der DGKJ (Hg.): Gesellschaft für Kinderheilkunde 1883-2008. Berlin: H. Heenemann, 17-35.

Sofer, L. (1910): Beiträge zur Rassenphysiologie und Rassenpathologie. Politisch Anthropologische Revue 9.

Steinkamp, G. (1999): Soziale Ungleichheit in Morbidität und Mortalität. Oder: Warum einige Menschen gesünder sind und länger leben als andere. In: Schlicht, W. und Dickhuth, H.H. (Hg.): Gesundheit für alle. Fiktion oder Realität? Schorndorf: Hoffmann, 101-154.

Swart, E. (2001): Sozial-Epidemiologie in der Lehre. In: Mielck, A. und Bloomfield, K. (Hg.): Sozial-Epidemiologie. Eine Einführung in die Grundlagen, Ergebnisse und Umsetzungsmöglichkeiten. Weinheim/München: Juventa-Verlag, 330-340.

Thomann, K.D. (1979): Die Zusammenarbeit der Sozialhygieniker Alfred Grotjahn und Alfons Fischer. Medizinhistorisches Journal 14(4): 251-274. 
Townsend, P. (1982): Inequalities in health. The Black report. Harmondsworth: Penguin.

Trojan, A. (2001): Soziale Stadtentwicklung und Armutsbekämpfung als Gesundheitsförderung. Das Gesundheitswesen 63 (Sonderheft 1): 43-47.

Weindling, P. (1984): Soziale Hygiene: Eugenik und medizinische Praxis. Der Fall Grotjahn. Das Argument 119: 6-20.

Weindling, P. (1985): Weimar Eugenics: The Kaiser Wilhelm Institute for Anthropology, Human Heredity and Eugenics in Social Context. Annals of Science 42: 303-318.

Weindling, P. (1987a): Die Verbreitung rassenhygienischen/eugenischen Gedankengutes in bürgerlichen und sozialistischen Kreisen in der Weimarer Republik. Medizinhistorisches Journal 22: 352-368.

Weindling, P. (1987): Medical practice in imperial Berlin. The casebook of Alfred Grotjahn. Bulletin of the History of Medicine 61(3): 391-410.

Weingart, P.; Kroll, J.; Bayertz, K. (1992): Rasse, Blut und Gene. Geschichte der Eugenik und Rassenhygiene in Deutschland. Frankfurt a.M.: Suhrkamp.

Wilkinson, R.G. (1992): Income distribution and life expectancy. British Medical Journal 304: 165-168. 


\section{Werthaltungen und ihre kritische Validierung}





\section{Konzeptionelle und methodische Probleme der Lebensqualitätsmessung als Grundlage der Bewertung der Ergebnisse medizinischer Maßnahmen}

Reinhold Kilian

\section{Einleitung}

Die Idee, Lebensqualität (LQ) zu messen und die Ergebnisse dieser Messung als Indikatoren für den Erfolg gesundheitsbezogener Interventionen $\mathrm{zu}$ verwenden, erscheint aus heutiger Perspektive so unmittelbar einleuchtend, dass die tatsächliche Entstehung dieser Idee in der Mitte des 20. Jahrhunderts auf den ersten Blick erstaunlich verspätet anmutet. Noch erstaunlicher, als das späte Auftauchen des Lebensqualitätsbegriffs in der Medizin, sind allerdings die Begleitumstände der Etablierung von Lebensqualität als wissenschaftliches Konzept und seiner rasanten Verbreitung in der medizinischen Wissenschaft.

Der Lebensqualitätsbegriff verdankt seine Geburt als wissenschaftliches Konzept nicht allein, wie vielfach kolportiert, einer Wahlkampfrede von Lyndon B. Johnson (Präsident der USA von 1963-1969), in der dieser seine Ziele als künftiger Präsident der USA mit den Worten beschrieb:

"The purpose of protecting the life of our Nation and preserving the liberty of our citizens is to pursue the happiness of our people. Our success in that pursuit is the test of our success as a Nation. For a century we labored to 
settle and to subdue a continent. For half a century we called upon unbounded invention and untiring industry to create an order of plenty for all of our people. The challenge of the next half century is whether we have the wisdom to use that wealth to enrich and elevate our national life, and to advance the quality of our American civilization." (Johnson 1964, S. 704-707) (zu finden unter: http://coursesa.matrix.msu.edu/ hst306/documents/ great.html, letzter Zugriff 27.06.2013)

Die Einführung des Lebensqualitätskonzeptes in die Wissenschaft erfolgte vielmehr durch den Ökonomen John Kenneth Galbraith, der als politischer Berater den Wahlkampf Johnsons unterstützte (Galbraith 1964, S. 117-123). In diesem Artikel, erschienen in der Zeitschrift Science unter dem Titel Economics and the Quality of Life (Galbraith 1964), zeichnet Galbraith das Bild einer Gesellschaft, die durch eine dramatische Steigerung ihrer ökonomischen Produktivität den Zwängen einer Priorisierung ökonomischer Ziele entwachsen ist und sich statt dessen der Steigerung ihrer Lebensqualität widmen kann. Lebensqualität im Sinne Galbraiths bedeutet dabei hauptsächlich die Förderung der Befriedigung kultureller und ästhetischer Bedürfnisse sowie die Emanzipation des Individuums vom Zwang der Unterordnung unter ökonomische Notwendigkeiten.

"Escape from the commitment to economic priority has, it will be clear, a broadly emancipating role. It enables us to consider a range of new tasks from the beautification of our cities the cleaning up of roadside commerce and advertising, the enlargement of cultural opportunity, the redemption of mass communications to the suppression of the influence of weapon makers on foreign policy“ (Galbraith 1964, S. 122).

Auch die Übernahme des Lebensqualitätsbegriffs durch die Medizin vollzog sich vor dem Hintergrund der Erkenntnis, dass mit der rapiden Entwicklung der medizintechnischen Behandlungsmöglichkeiten in der zweiten Hälfte des 20. Jahrhunderts eine ebenso dramatische Zunahme von Entscheidungsproblemen, sowohl für die behandelnden Ärzte als auch für die für die Allokation von 
Ressourcen verantwortlichen gesellschaftlichen Institutionen einher ging (Elkinton 1966). Ähnlich wie für die Ökonomie, zeigt sich auch in den Begründungen für die Notwendigkeit einer stärkeren Berücksichtigung der Lebensqualität durch die Medizin die Einschätzung, dass der technische Fortschritt die Leistungsfähigkeit der medizinischen Versorgung zwar deutlich gesteigert hat, dass jedoch dabei das eigentliche Ziel der Medizin aus dem Blick geraten ist (Elkinton 1966). Als einer der ersten Mediziner, die den Begriff der Lebensqualität in die medizinische Literatur eingeführt haben, verweist Elkinton dabei auf Francis Bacon:

"'The office of medicine`, said Francis Bacon, ,is but to tune this curious harp of man's body and reduce it to harmony. Almost 4 centuries later, in the accelerating scientific revolution that Francis Bacon fathered, medicine is doing the tuning with unprecedended skill but is having trouble with the harmony." (Elkinton 1966, S. 711)

Die von Francis Bacon als Aufgabe der Medizin betrachtete Herstellung der Harmonie wird von Elkinton unmittelbar in den Begriff der Lebensqualität überführt »What is the harmony within a man, and between man and his world - the quality of life - to which the patient, the physician and the society aspires?« (Elkinton 1966, S. 711)

Bereits bei Elkinton wird allerdings die Schwierigkeit deutlich, den Begriff der Lebensqualität ontologisch und epistemologisch so $\mathrm{zu}$ verorten, dass er als wissenschaftliches Konstrukt operationalisierbar wird. Am Ende seines Artikels delegiert Elkinton die Aufgabe dieser Definition zurück an die Ärzte:

"Just what constitutes this quality of life for a particular patient, and the therapeutic pathway to it, often is extremely difficult to judge and must lie with the conscience of the physician, but surely such quality of life is the harmony of which Francis Bacon spoke." (Elkinton 1966, S. 713)

Möglicherweise hätte sich Elkinton mit der Definition des Lebensqualitätsbegriffs in Anlehnung an Bacon leichter getan, wenn er 
diesen nicht an dessen metaphorischen humoralpathologisch gefärbten Harmoniebegriff, sondern an seine konkrete Kritik der Medizin seiner Zeit geknüpft hätte. So war Bacon keineswegs ein Kritiker des medizinischen Fortschritts, sondern betrachtete das fehlende Interesse der Mediziner an einer wissenschaftlich fundierten Verbesserung ihrer Behandlungsmethoden als eines der zentralen Defizite. Gravierender als dieses fehlende Interesse am wissenschaftlichen Fortschritt war für Bacon aber die Tatsache, dass die Mediziner seiner Zeit die Beschränkungen ihrer Behandlungsmethoden als naturgegeben betrachteten und Krankheiten, die sie nicht behandeln konnten, als »unheilbar« erklärten. Darüber hinaus wurde die vermeintliche Unheilbarkeit von Erkrankungen als Rechtfertigung dafür verwendet, sich um die Leiden dieser als unheilbar erklärten Patienten nicht länger zu kümmern (Boss 1978).

Ausgehend von dieser Kritik sah Bacon die Aufgabe der Medizin nicht nur in der Anwendung vorhandener Kenntnisse zur Heilung von Krankheiten

"... but also the extension of that knowledge, and this not only for reasons of principle [...] but also because of the personal responsibility of the physician towards the patient. The aims of $m$ edicine must also include, beside the restoration of health, the relief of pain and suffering, not only when it contributes to recovery but even at the approach to death" (Boss 1978, S. 215).

Mit dieser Definition der Ziele der medizinischen Behandlung übernimmt Bacon eine Idee, die bereits Thomas More ein Jahrhundert vorher in seinem 1516 erschienen Werk Utopia formuliert hat: Es gehört zum Wesen einer zivilisierten Gesellschaft, nicht nur die Kranken, bei denen Heilungsaussichten bestehen, zu behandeln und zu pflegen, sondern auch diejenigen, für die es keine Behandlungsmöglichkeiten gibt, zu trösten und ihnen bei der Linderung ihrer Leiden zu helfen (Kilian und Angermeyer 1999). So lässt More den Erzähler seines fiktiven Reiseberichts über die Behandlung von Kranken durch die Bewohner von Utopia sagen: 
"I have already told you with what care they look after their sick, so that nothing is left undone that can contribute either to their ease or health: and for those who are taken with fixed and incurable diseases, they use all possible ways to cherish them, and to make their lives as comfortable as possible. They visit them often, and take great pains to make their time pass off easily." (More 1901, S. 68) (zu finden unter: http://oregonstate.edu/instruct/phl302/texts/more/utopia-slaves.html, letzter Aufruf 27.06.2013).

Sowohl bei More als auch bei Bacon sind nicht nur Grundlagen für die moderne Palliativmedizin, sondern auch grundlegende Aspekte der Definition des Konzeptes der Lebensqualität in der Medizin erkennbar und zwar die Reduzierung des mit dem Auftreten von Erkrankung verbundenen Leids unabhängig von den Heilungsmöglichkeiten. Allerdings wird Lebensqualität hier nicht im Sinne eines messbaren Konstrukts beschrieben, sondern als ontologisches Prinzip, das die Freiheit von Schmerz und Leid als Voraussetzung für ein menschenwürdiges Leben ansieht.

Ontologisch ließe sich ein Lebensqualitätsbegriff, der in der Tradition von More und Bacon als Reduzierung von krankheitsbedingtem Leiden definiert wird, als epikureisch bzw. hedonistisch und, in seiner staatsphilosophischen Anwendung, als utilitaristisch verorten (Henderson und Knight 2012). Auch wenn es im Einzelfall schwierig sein mag, das individuelle, durch eine Erkrankung verursachte Ausmaß an Leid zu bestimmen, scheint ein hedonistisch definierter Lebensqualitätsbegriff einer Messung relativ leicht zugänglich zu sein. Wenn ein Leben mit weniger Leid ein besseres Leben ist als ein Leben mit viel Leid, gilt es im Sinne der Messtheorie von Stevens (Stevens 1946), nur noch einen Weg zu finden, die Summe individuellen Leids einer Zahl zuzuordnen. Dabei wäre es unerheblich, ob diese Zuordnung die Anforderung eines metrischen oder »nur« eines ordinalen Messniveaus erfüllt, es müsste lediglich sichergestellt sein, dass ein höherer Zahlenwert immer entweder mehr oder weniger Leid, und damit mehr oder weniger Lebensqualität, repräsentiert als ein niedrigerer (Stevens 1946). 
Zentral für ein hedonistisch verortetes Lebensqualitätskonzept ist dabei die subjektive Perspektive des Individuums. Leid ist ebenso wie Freude per definitionem eine subjektive, allerdings nicht notwendigerweise idiosynkratische Erfahrung. Die Messung von Leid oder Freude, und demzufolge einer auf diesen Erfahrungen basierenden Lebensqualität, kann nicht unmittelbar objektiv, sondern nur mittelbar über die Äußerungen des Individuums erfolgen (Diener 2000).

Einer derartigen eindeutigen ontologischen Verortung des Lebensqualitätsbegriffs widerspricht allerdings die Tatsache, dass Menschen zu allen Zeiten bereit waren, für die Verwirklichung höherer Ideale wie z.B. Tugend, Tapferkeit, Gerechtigkeit, Wahrheit, Freiheit oder Religion, den Verzicht auf Lust und Freude oder das Erleben von Leid und Schmerz in jeglicher Form in Kauf zu nehmen. Offensichtlich bildet das Streben nach Lust oder nach einer Vermeidung von Leid nur eine Dimension der menschlichen Existenz, während eine andere Dimension in dem Streben nach der Erfüllung einer gattungsspezifischen Bestimmung liegt (Bayertz 2010; Henderson und Knight 2012). In der antiken Philosophie wurde insbesondere von Aristoteles das Streben nach dem guten, d.h. tugendhaften Leben, als Bestimmung des Menschen und damit als Grundlage für die Erreichung eines glücklichen Lebens angesehen (Bayertz 2010; Henderson und Knight 2012). Eine ontologische Verortung des Lebensqualitätsbegriffes auf der Basis eines derartigen, als eudämonisch bezeichneten, teleologischen Prinzips würde beinhalten, dass Lebensqualität grundsätzlich einer objektiven Messung zugänglich wäre. Gleichzeitig würde sie im Gegensatz zum hedonistischen Lebensqualitätsbegriff grundsätzlich die Möglichkeit implizieren, dass Menschen ihre eigene Lebensqualität falsch beurteilen, oder zumindest die eigene Einschätzung ihrer Lebensqualität von der Einschätzung ihrer Lebensqualität durch andere Menschen abweicht (Bayertz 2010).

Die vermeintlich objektive Beurteilung der Lebensqualität eines Menschen ist allerdings nur unter der Maßgabe allgemein geteilter Vorstellungen darüber möglich, was letztlich ein gutes Leben ausmacht (Henderson und Knight 2012). Da zumindest in den westlich geprägten modernen Gesellschaften eine der zentralen Wertvorstel- 
lungen in der Anerkennung des Pluralismus von Ansichten über die Voraussetzungen eines guten Lebens besteht, erweist sich die moderne Definition der Zielkriterien eudämonischer Lebensqualitätskonzepte als ziemlich komplex.

"This led to the emergence of a variety of theories and approaches, with personal growth and meaning in life often emphasized as core eudaimonic components. [...] Other constructs that have also been linked to the eudaimonic perspective include purpose, autonomy, competence, self-realization, mindfulness, self-acceptance, authenticity, values congruence and social connectedness" (Henderson und Knight 2012, S. 198).

Angesichts eines derartig breiten Spektrums an theoretischen Konstrukten mit diesen inhärenten definitorischen Variationsmöglichkeiten erweist sich die epistemologische Basis für eine objektive Messung der eudämonischen Lebensqualität in modernen Gesellschaften als äußerst fragil.

Ziel dieses Beitrags ist es, eine kurze Übersicht darüber zu geben, in welcher Weise versucht wurde, das Problem der Definition und der Operationalisierung des Lebensqualitätskonstruktes als Ergebniskriterium für die Bewertung medizinischer Maßnahmen zu lösen und welche methodischen Probleme sich hierbei ergeben haben. Angesichts der Fülle der Veröffentlichungen zu der Thematik erhebt der Beitrag keinen Anspruch auf Vollständigkeit, sondern versucht lediglich, einige zentrale Entwicklungslinien aufzuzeigen und im Hinblick auf ihre Bedeutung für die aktuelle Anwendung des Lebensqualitätskonzeptes in der Medizin zu diskutieren.

\section{Ansätze zUr Messung von Lebensqualität}

In der modernen Lebensqualitätsforschung wird das Problem der ontologischen Verortung des Lebensqualitätsbegriffes sehr unterschiedlich gehandhabt. In der unter anderem auf die Argumentation von Galbraith zurückgehenden sozialwissenschaftlichen Le- 
bensqualitätsforschung wurden seit den 1970 er Jahren zunächst in den USA qualitative Untersuchungen zu der Frage durchgeführt, was Menschen unter Lebensqualität verstehen und welche Aspekte ihres Lebens für ihre Lebensqualität von Bedeutung sind (Andrews und Withey 1976). Aus den Ergebnissen dieser Untersuchungen entwickelten Andrews und Withey (1976) eine 7-stufige Likert-Skala, auf der Befragte verschiedene Dimensionen ihres Lebens beurteilen sollten (Andrews und Withey 1976). Diese nach den Extremkategorien der Antwortskala als »Delighted to Terrible« bezeichnete Skala wurde in zahlreiche Sprachen übersetzt und international zur Erfassung der Lebensqualität in der Allgemeinbevölkerung eingesetzt.

Während die sozialwissenschaftlich orientierte Lebensqualitätsforschung eher auf eine möglichst vollständige Repräsentation aller für die Lebensqualität eines Menschen relevanten Lebensbereiche abzielte, versuchten Vertreter einer eher an psychologischen Konzepten ausgerichteten LQ-Forschung kognitive und emotionale Dimensionen des menschlichen Erlebens in Modellen subjektiven Wohlbefindens zu verknüpfen (Cummins 1996; Cummins 2000; Cummins et al. 2002; Cummins 2005; Diener 1984; Diener et al. 1985; Diener 2000). Auch diese Verfahren bedienen sich in der Regel der Messung über Ratingskalen, wie z.B. der Satisfaction with Life Scale (Diener et al. 1985; Diener 2000).

In der Ökonomie findet sich demgegenüber eine spezifische Form des hedonistischen Lebensqualitätskonzeptes, welche auf utilitaristisch geprägten wohlfahrtstaatlichen Prinzipien basiert und die Erfassung des subjektiven Nutzens als zentrales Kriterium der Lebensqualitätsmessung betrachtet (Schöffski et al. 1998; von Neumann und Morgenstern 1953). Die nutzentheoretisch fundierte Lebensqualitätsmessung basiert auf der Spieltheorie ökonomischen Verhaltens, die von Neumann und Morgenstern (von Neumann und Morgenstern 1953) entwickelt wurde. Nach dieser Theorie lässt sich subjektive Lebensqualität ausschließllich über die Erfassung von Entscheidungspräferenzen messen: 
"It is clear that every measurement - or rather every claim of measurability - must be ultimately based on some immediate sensation, which possibly cannot and certainly need not be analyzed any further. In the case of utility the preference - of one object or aggregate of objects as against another provides this basis" (von Neumann und Morgenstern 1953, S. 16).

Abgeleitet aus diesem allgemeinen messtheoretischen Prinzip wird Lebensqualität aus dieser Perspektive als der Nutzwert betrachtet, den ein Mensch seiner aktuellen Lebenssituation beimisst. Die Bestimmung dieses Nutzwertes erfolgt durch die Erfassung des Präferenzverhaltens von Menschen in hypothetischen Entscheidungssituationen (Schöffski et al. 1998).

\section{Ansätze ZUR LebensqualitätSMESSUNG IN DER MEDIZIN}

Trotz der oben bereits diskutierten definitorischen Unklarheiten wurde die Lebensqualität als Kriterium für die Bewertung medizinischer Leistungen innerhalb von nur zwei Jahrzehnten in allen medizinischen Teildisziplinen weitgehend akzeptiert (Bowling 2001; Krupinski 1980; Ware, Jr. 1984; Ware, Jr. 1991; Ware, Jr. 2003). Die Zahl und die inhaltliche Vielfalt der Methoden zur Messung gesundheitsbezogener Lebensqualität ist mittlerweile so unüberschaubar groß (Angermeyer et al. 2000; Bowling 2001), dass an dieser Stelle nur einige wichtige Entwicklungslinien aufgezeigt werden können.

So lassen sich zum einen Ansätze zur generischen Erfassung gesundheitsbezogener Lebensqualität wie z.B. der Short Form (SF) 36 (Ware, Gandek, the IQOLA Project Group 1994) und der World Health Organization Quality of Life (WHOQOL) 100 (Angermeyer et al. 2000; The WHOQOL Group 1998b) von erkrankungsspezifischen Verfahren z.B. für Patienten mit Krebs (Aaronson et al. 1993), Diabetes (Jacobson 1997; Jacobson et al. 1994) oder psychischen Erkrankungen (Lehman 1997; Lehman 2006) unterscheiden. 
Ein weiteres Unterscheidungsmerkmal bildet die Fokussierung auf die durch die Erkrankung bedingte Beeinträchtigung körperlicher Funktionen wie z.B. im SF36 (Ware et al. 1994) oder auf die Zufriedenheit mit bzw. die Bewertung von Lebensbedingungen wie z.B. in den WHOQOL Instrumenten (Angermeyer et al. 2000; The WHOQOL Group 1998a; The WHOQOL Group 1998b).

Schließlich lassen sich alle bisher genannten, auf einer psychometrischen Messtheorie basierenden Verfahren von Verfahren abgrenzen, die auf der Basis der oben dargestellten nutzentheoretischen Messtheorie entwickelt wurden und die primär für gesundheitsökonomische Analysen verwendet werden (Schöffski et al. 1998). Hier ist insbesondere der EuroQol (EQ5D) zu nennen (Brooks et al. 2003).

Das oben skizzierte Problem der ontologischen Verortung des Lebensqualitätskonzeptes wird im Zusammenhang mit der Entwicklung von Verfahren zur Erfassung der gesundheitsbezogenen Lebensqualität eher selten diskutiert. Allerdings lässt sich aus der inhaltlichen Ausrichtung einiger Instrumente eine entsprechende Verortung durchaus entnehmen. So entspricht eine weitgehende Fokussierung auf Aspekte der funktionalen Beeinträchtigung, wie z.B. im SF-36 eher einer eudämonischen Definition des Lebensqualitätskonzeptes, während Instrumente, die sich eher auf Aspekte des subjektiven Wohlbefindens und der subjektiven Bewertung von Lebensbedingungen richten, wie z.B. die WHOQOL-Instrumente, aber auch die nutzentheoretisch fundierten Instrumente, eher einem hedonistischen Lebensqualitätskonzept entsprechen.

Im Hinblick auf eine Verwendung des Lebensqualitätskonzeptes als Kriterium für die Bewertung medizinischer Maßnahmen gewinnt das Problem der ontologischen Verortung des Lebensqualitätsbegriffs seine praktische Bedeutung dadurch, dass mit der gewählten Perspektive jeweils sowohl spezifische Erkenntnismöglichkeiten aber auch spezifische methodische und konzeptionelle Probleme verbunden sind.

So ermöglicht es die auf dem eudämonischen Prinzip basierende Messung funktionaler Beeinträchtigungen, z.B. mit dem SF-36, 
Aussagen darüber zu machen, welche Auswirkungen eine bestimmte Form der Behandlung, z.B. eine Chemotherapie bei Brustkrebs, nicht nur auf die physische Befindlichkeit der Patientin, sondern auch auf ihr psychisches Wohlbefinden und die Qualität ihrer sozialen Beziehungen hat. Mit dem Fokus auf die funktionale Beeinträchtigung bleibt der Ansatz des SF-36 und vergleichbarer Instrumente aber der Vorstellung verhaftet, dass die Qualität menschlichen Lebens an die Fähigkeit zur Erfüllung körperlicher, geistiger und sozialer Funktionen gebunden ist. Aus dieser Verknüpfung von Lebensqualität und Funktion ergibt sich allerdings die notwendige Konsequenz, dass jede Einschränkung funktioneller Kapazitäten zwangsläufig mit einer reduzierten Lebensqualität verbunden ist und dass dort, wo Krankheit zu einem endgültigen Verlust funktionaler Kapazitäten führt, keine Verbesserung der Lebensqualität mehr möglich ist. Ein derartiges Verständnis widerspricht jedoch nicht nur den bereits von More und Bacon formulierten Vorstellungen über die Aufgaben der Medizin bzw. der zivilisierten Gesellschaft, sondern auch modernen rehabilitations- und palliativmedizinischen Konzepten. So basiert die Neufassung der Internationalen Klassifikation Funktionsfähigkeit, Behinderung und Gesundheit (ICF) (World Health Organization 2001) noch dezidierter als ihre Vorläufer auf einer Unterscheidung zwischen funktionalen Beeinträchtigungen, Fähigkeiten und sozialer Partizipation. Grundlage dieser Differenzierung ist die Vorstellung, dass Beeinträchtigungen körperlicher Funktionen erst im Zusammenwirken mit Faktoren der sozialen und physikalischen Umwelt zur Beeinträchtigung von Aktivitäten und sozialer Partizipation führen. Damit wird der Überzeugung Rechnung getragen, dass eine zentrale Aufgabe zivilisierter Gesellschaften darin besteht, Lebensbedingungen zu schaffen, die auch bei einer Beeinträchtigung funktionaler Kapazitäten nicht zwangsläufig zu einem Verlust von Lebensqualität führen. Für die Definition und die Messung von Lebensqualität ergibt sich aus diesem Anspruch die Notwendigkeit einer Trennung funktionaler Dimensionen von Dimensionen des subjektiven Erlebens und der subjektiven Bewertung (Angermeyer et al. 2000; The WHOQOL Group 
1998b). Konzepte und Operationalisierungen von Lebensqualität, die diese Anforderung erfüllen, lassen sich überwiegend eher einer hedonistischen Lebensqualitätsdefinition zurechnen (Henderson und Knight 2012). So definiert Diener (Diener 2000) das von ihm als Synonym für subjektive Lebensqualität verwendete Konzept des subjektiven Wohlbefindens explizit in Abgrenzung zu einem eudämonischen Lebensqualitätsbegriff:

"For millenia thinkers have pondered the question, what is a good life? They have focused on criteria such as loving others, pleasure, or self-insight as the defining characteristics of quality of life. Another idea of what constitutes quality of life, however, is that it is desirable for people themselves to think that they are living good lives. This subjective definition of quality of life is democratic in that it grants to each individual the right to decide whether his or her life is worthwhile. It is this approach to defining the good life that has come to be called subjective well-being, (SWB) and in colloquial terms is sometimes labeled happiness. SWB refers to people's evaluations of their lives - evaluations that are both affective and cognitive" (Diener 2000, S. 34).

Auch die den WHOQOL-Instrumenten zur Lebensqualitätsmessung zugrundeliegende Lebensqualitätsdefinition der WHO betrachtet die subjektive Bewertung der eigenen Lebenssituation als zentral:

"Lebensqualität ist die individuelle Wahrnehmung der eigenen Lebenssituation im Kontext der jeweiligen Kultur und des jeweiligen Wertesystems und in Bezug auf die eigenen Ziele, Erwartungen, Beurteilungsmaßstäbe und Interessen" (Angermeyer et al. 2000, S. 11).

Im Unterschied zu dem Modell des subjektiven Wohlbefindens beinhaltet der WHOQOL-Ansatz allerdings neben der subjektiven Bewertung sowohl funktionale als auch materielle Grundlagen subjektiven Wohlbefindens und stellt damit den Versuch einer Integration hedonistischer und eudämonischer Lebensqualitätskonzepte 
dar (Angermeyer et al. 2000; Henderson und Knight 2012; Power et al. 1999; Skevington 2002; The WHOQOL Group 1998a; The WHOQOL Group 1998b).

Auch nutzentheoretisch fundierte Instrumente zur Erfassung der Lebensqualität beinhalten den Versuch einer Kombination hedonistischer und eudämonischer Konzepte. So werden mit dem EuroQol (EQ-5D) Fragebogen krankheitsbedingte funktionale Beeinträchtigungen als Gesundheitszustände (health states) auf fünf Dimensionen erfasst und in einem zweiten Schritt im Hinblick auf die durch sie verursachten Nutzwertverluste bewertet (Szende et al. 2007). Die Bewertung erfolgt dabei entweder über eine visuelle Analogskala oder über spezielle Verfahren, in denen Befragte ihre Präferenzen für das Erreichen eines guten Gesundheitszustandes gegen das Risiko eines vorzeitigen Todes abwägen müssen (Brooks et al. 2003; Schöffski et al. 1998; Szende et al. 2007). Nutzentheoretisch fundierte Instrumente zur Lebensqualitätsmessung werden nahezu ausschließlich zur Bestimmung qualitätsadjustierter Lebensjahre (QALYs) im Rahmen gesundheitsökonomischer Untersuchungen eingesetzt.

Mit der vollständigen oder teilweisen Loslösung des Lebensqualitätsbegriffs von Aspekten der funktionalen Kapazität ermöglichen es Konzepte wie das des subjektiven Wohlbefindens grundsätzlich zu untersuchen, wie sich medizinische Maßnahmen unabhängig von ihrer klinischen Wirksamkeit auf die Lebensqualität von Menschen auswirken. In der empirischen Umsetzung dieses Potenzials zeigt sich jedoch bereits sehr früh, dass Menschen offenbar über sehr weitreichende Fähigkeiten zur Anpassung ihres individuellen Anspruchsniveaus an ihre äußeren Lebensbedingungen einschließlich ihrer biologischen Körperfunktionen verfügen (Angermeyer und Kilian 2006; Cummins 2000; Cummins et al. 2002; Cummins und Nistico 2002; Cummins 2005; Diener und Diener 1996). So zeigen zahlreiche Untersuchungen, dass Menschen auch dann ein hohes Maß an subjektivem Wohlbefinden berichten, wenn sie mit extrem schlechten Lebensbedingungen, wie z.B. schweren 
Erkrankungen, konfrontiert sind (Angermeyer und Kilian 2006; Diener und Diener 1996).

Während dieses Phänomen aus methodischer Sicht unter dem Begriff »response-shift« als methodisches Artefakt behandelt wird (Schwartz und Sprangers 1999; Sprangers und Schwartz 1999; Wilson 1999), sind verschiedene Autoren in ihrer theoretischen Aufarbeitung der diesbezüglichen Forschungsergebnisse zu dem Schluss gekommen, dass das Erleben subjektiven Wohlbefindens Ergebnis eines homöostatischen Prozesses bildet, durch den Menschen ihre emotionale Stabilität unter dem Einfluss wechselnder Umweltbedingungen regulieren (Angermeyer und Kilian 2006; Cummins 2000; Cummins et al. 2002; Cummins und Nistico 2002; Cummins 2005).

Die Grundlage dieses homöostatischen Prozesses sehen viele Autoren darin, dass die Aufrechterhaltung eines bestimmten Maßes an subjektivem Wohlbefinden ein allgemeines menschliches Bedürfnis darstellt. Diener und Diener sehen in dieser positiven menschlichen Grundtendenz sogar einen phylogenetischen Vorteil:

"[...] it is important for motivational reasons that people not be in a negative mood most of the time. Approach tendencies must prevail in behavior for people to obtain food, shelter, social support, sex, and so forth. Because positive moods energize approach tendencies, it is desirable that people on average be in a positive mood. Human approach tendencies are manifest in the rapid exploration and settlement of new frontiers and in the unremitting invention of new ideas and institutions throughout human history. Thus, not only might humans' large brains and opposable thumbs be responsible for the rapid spread of humanity across the globe, but positive emotions might also be an important factor. Finally, a positive set point may motivate human sociability, drive-free exploration, and creativity, and produce a strong immune response to infections" (Diener und Diener 1996, S. 184).

Was in Diener und Dieners Argumentation fehlt, ist allerdings der Hinweis darauf, dass sich dieser phylogenetische Vorteil nur entfalten kann, wenn die dauerhafte Aufrechterhaltung subjektiven Wohl- 
befindens nicht vollständig gelingt, da ansonsten jede Motivation zur Veränderung von Umweltbedingungen erlöschen würde. Dementsprechend zeigen die von den Autoren zur Unterstützung ihrer Hypothese herangezogenen Studienergebnisse, dass Menschen ihr subjektives Wohlbefinden zwar meistens positiv bewerten, dass die Bewertung jedoch nur selten den Zustand vollständigen Wohlbefindens erreicht. Auf einer Skala zwischen o für die niedrigste und 100 für die höchste Ausprägung subjektiven Wohlbefindens liegen die in internationalen Studien ermittelten Durchschnittswerte zwischen 54 und 79, wobei die Werte im Zeitverlauf nur wenig schwanken (Diener und Diener 1996).

Es spricht also durchaus einiges dafür, dass es ein Basisniveau menschlichen Wohlbefindens gibt, welches niedrig genug ist, um die Motivation zur Verbesserung der Lebensbedingungen aufrecht zu erhalten, welches aber gleichzeitig hoch genug ist, um die Hoffnung aufrecht zu erhalten, dass Aktivitäten zur Verbesserung der Lebensbedingungen auch erfolgreich sind (Cummins et al. 2002; Angermeyer und Kilian 2006). Dieses Basisniveau menschlichen Wohlbefindens ist allerdings keineswegs als statische Größe zu verstehen, sondern vielmehr als Steuerungsmechanismus (ähnlich wie die Körpertemperatur) eines homöostatischen Systems, dessen Ziel in der Aufrechterhaltung einer möglichst guten Balance zwischen menschlichen Wünschen und Ansprüchen und den Möglichkeiten ihrer Erfüllung besteht (Cummins et al. 2002).

Voraussetzung eines derartigen homöostatischen Prozesses ist die menschliche Fähigkeit, eigene emotionale Reaktionen in bestimmtem Umfang durch kognitive Aktivitäten zu beeinflussen. Die Erkenntnis der Bedeutung der Fähigkeit des Menschen zur Anpassung seiner Wünsche und Ziele an das Erreichbare bzw. an das Unvermeidbare ist keineswegs neu, sondern wird bereits in der Antike durch die Stoiker als bester Weg zur Erreichung eines glücklichen Lebens angesehen. In allegorischer Form findet sich diese Perspektive z.B. in der Äsop zugeschriebenen Fabel vom Fuchs, der, 
als er die von ihm begehrten Trauben nicht erreichen kann, sich mit der Annahme tröstet, diese seien wahrscheinlich sowieso sauer.

Für die Anwendung als Ergebniskriterium zur Bewertung medizinischer Maßnahmen ergibt sich aus dem Phänomen des »response-shift« bzw. der Lebensqualitätshomöostase das Problem, dass unklar ist, inwieweit ein Zustand, der primär über individuelle kognitive Prozesse gesteuert wird, durch äußere Einflüsse überhaupt beeinflusst werden kann. Wenn Menschen also einerseits dazu in der Lage sind, ein bestimmtes Niveau subjektiven Wohlbefindens auch unter dem Einfluss negativer Umwelteinflüsse (wie z.B. Krankheit) aufrechtzuerhalten, wie sollte es dann möglich sein, das subjektive Wohlbefinden durch eine Veränderung dieser Umweltbedingungen (z.B. durch eine medizinische Behandlung) zu steigern. Es stellt sich somit die Frage, ob die Messung der Wirksamkeit medizinischer Interventionen mit dem Konzept der subjektiven Lebensqualität überhaupt möglich bzw. sinnvoll ist (Pukrop 2002).

Antworten auf diese Frage liefern genauere Analysen des Prozesses der Wohlbefindenshomöostase, wie sie von der Arbeitsgruppe des australischen Psychologen Robert A. Cummins durchgeführt wurden. So bestätigen die Untersuchungsergebnisse von Cummins, dass die meisten Menschen über eine weitreichende Fähigkeit verfügen, ihr subjektives Wohlbefinden durch Anpassung ihres Anspruchsniveaus unter wechselnden Umweltbedingungen aufrecht zu erhalten. Allerdings weist das Niveau subjektiven Wohlbefindens dabei immer eine Variationsbreite auf, die sich im Erleben reduzierten bzw. gesteigerten Wohlbefindens äußert:

"The general idea oft the proposed homeostatic system is that SWB [subjective well being; ergänzt durch RK] is managed, for each individual, within a set point range، (Cummins 2000). That is, each individual has an in-built set point for their normal level of SWB [...] and their perceived SWB is normally held within a narrow range around this setting. This idea also involves the concept of threshold, as previously discussed, which we propose exists at the margins of the set-point range. That is, as SWB approaches these 
margins the homeostatic system resists further change and, if the threshold is exceeded, the homeostatic system then works to bring SWB back to lie within the normal range for the individual person" (Cummins et al. 2002, S. 12).

D.h. das System der Wohlbefindenshomöostase bietet durchaus Spielraum für das Erleben einer Beeinträchtigung bzw. Steigerung des subjektiven Wohlbefindens, allerdings nur im Rahmen einer bestimmten Variationsbreite, die Cummins et al. im Bereich von 2 Standardabweichungen ansiedeln (Cummins et al. 2002).

Für die Frage der Verwendbarkeit von subjektiver Lebensqualität als Ergebniskriterium bedeutet dies, dass unter dem Einfluss von Krankheitssymptomen durchaus mit einer Beeinträchtigung der subjektiven Lebensqualität zu rechnen ist, dass diese Beeinträchtigung jedoch in der Regel nicht dauerhaft unter ein bestimmtes Niveau fällt. Gleichzeitig kann damit gerechnet werden, dass eine Linderung oder Beseitigung der Krankheitssymptome zur Wahrnehmung einer dauerhaften Steigerung der subjektiven Lebensqualität nur bis zu einem bestimmten Niveau führt.

Allerdings verweisen Cummins et al. auch darauf, dass das System der menschlichen Wohlbefindenshomöostase durch extreme Umweltanforderungen auch überfordert werden kann.

"However, all homeostatic systems have their limitations an so it would also be expected that SWB recovery would be contingent on the residual discomfort or lost functional status not being overly severe" (Cummins et al. 2002, S. 12).

Als Beispiel führen die Autoren eine Untersuchung bei Paraplegiepatienten an, bei denen sich die Aufrechterhaltung eines »normalen« Niveaus subjektiven Wohlbefindens nur dann feststellen ließ, wenn die selbständige Atemfunktion der Patienten erhalten blieb, während ein dauerhafter Verlust der selbständigen Atemfunktion auch zu einer dauerhaften Beeinträchtigung des subjektiven Wohlbefindens führte (Cummins et al. 2002). 
Aus diesen Ergebnissen lässt sich schließen, dass extreme Umweltanforderungen, wie z.B. schwere Erkrankungen die menschliche Fähigkeit zur Aufrechterhaltung eines bestimmten Niveaus subjektiven Wohlbefindens überfordern können und dass demzufolge die Linderung dieser Umweltanforderungen durch medizinische Behandlung dazu beitragen kann, Menschen wieder in die Lage $\mathrm{zu}$ versetzen, ihr Wohlbefindensniveau selbst $\mathrm{zu}$ regulieren.

Subjektive Lebensqualität kann damit sehr wohl als Indikator für die Schwere einer gesundheitlichen Beeinträchtigung als auch für die Wirksamkeit einer medizinischen Behandlung herangezogen werden. Allerdings ergeben sich aus dem Phänomen der Wohlbefindenshomöostase sowohl theoretische als auch methodische Anforderungen an die Konzeption einschlägiger Untersuchungen. So erfordert die Verwendung subjektiver Lebensqualität als Indikator für die Schwere gesundheitlicher Beeinträchtigungen die Einbeziehung von Überlegungen zur Rolle zeitlicher und personeller Faktoren, welche den Prozess der Wohlbefindenshomöostase beeinflussen können. So lässt sich grundsätzlich davon ausgehen, dass homöostatische Prozesse umso mehr Zeit benötigen, je größer die jeweilige Anpassungsleistung ist, die erbracht werden muss. So ist bei plötzlich eintretenden gesundheitlichen Beeinträchtigungen, wie z.B. nach einem Unfall, kurzfristig mit einer stärkeren Beeinträchtigung des subjektiven Wohlbefindens zu rechnen als bei einer sich im Verlauf einer chronischen Erkrankung allmählich steigernden Beeinträchtigung. Gleichzeitig liegen Hinweise darauf vor, dass der Prozess der Wohlbefindenshomöostase durch Persönlichkeitsmerkmale beeinflusst wird (Cummins et al. 2002). Theoretische Modelle zu den Auswirkungen von medizinischen Behandlungen auf die subjektive Lebensqualität müssen dabei immer den systemischen, d.h. durch dynamische Wechselwirkungen geprägten, Charakter der Beziehungen zwischen den verschiedenen Einflussfaktoren der jeweils untersuchten Wirkungsbeziehung berücksichtigen.

Aus methodischer Sicht sind für die Überprüfung derart komplexer dynamischer Wechselwirkungsbeziehungen Längsschnitt- 
studien mit in der Regel mehr als drei Messzeitpunkten und den sich daraus ergebenden Datenstrukturen angemessene statistische Auswertungsmethoden, wie z.B. mixed-effects oder growth-curve Regressionsmodelle notwendig (Bollen und Curran 2006; Kilian et al. 2004; Kilian et al. 2012; Langle et al. 2012).

\section{Schlussbetrachtung}

Obwohl die Idee der Erfassung subjektiver Lebensqualität als Kriterium für die Bewertung medizinischer Maßnahmen erst in der zweiten Hälfte des 20. Jahrhunderts entwickelt wurde, reichen die theoretischen Wurzeln dieser Idee bis in die Antike. In diesen frühen theoretischen Grundlagen bereits auftretende Kontradiktionen und die sich daraus ergebenden konzeptionellen und methodischen Probleme sind bis heute nicht zufriedenstellend gelöst.

So steht der Anspruch, Lebensqualität auf der Grundlage subjektiver individueller Bewertungsmaßstäbe zu messen, der Anforderung gegenüber, aus diesen Messungen intersubjektive Aussagen über die objektive Wirksamkeit von medizinischen Behandlungsmethoden ableiten zu können. Die menschliche Fähigkeit zur Anpassung des Anspruchsniveaus und das daraus resultierende Phänomen des »response-shift « macht eine eindeutige Interpretation der Ergebnisse von Lebensqualitätsmessungen äußerst schwierig. Ob die von einigen Autoren geforderte und in einigen der dargestellten Ansätze zumindest teilweise verwirklichte Integration ursprünglich kontradiktorischer ontologischer Konzepte zur Überwindung dieser Probleme beiträgt, lässt sich gegenwärtig nur schwer beurteilen, da diese Probleme in der Mehrzahl von Untersuchungen nicht thematisiert werden.

Demgegenüber zeigt die jährlich steigende Zahl von Publikationen zum Thema Lebensqualität im Zusammenhang mit medizinischer Behandlung, dass die Lebensqualität als Zielkriterium zur Bewertung des Erfolgs medizinischer Maßnahmen mittlerweile in nahezu allen Bereichen der medizinischen Versorgung fest etabliert 
ist. ${ }^{1}$ Dieser anhaltende Erfolg lässt eine weitergehende Beschäftigung mit den konzeptionellen und methodischen Problemen der Lebensqualitätsforschung äußerst lohnenswert erscheinen. Anknüpfungspunkte ergeben sich dabei zum einen im Hinblick auf die Integration unterschiedlicher theoretischer Konzepte und zum anderen im Hinblick auf die Weiterentwicklung von Mess- und Auswertungsverfahren.

\section{LITERATUR}

Aaronson, N.K.; Ahmedzai, S.; Bergman, B.; Bullinger, M.; Cull, A.; Duez, N.J.; Filiberti, A.; Flechtner, H.; Fleishman, S.B.; de Haes, J.C.; Kaasa, S.; Klee, M.; Osoba, D.; Razavi, D.; Rofe, P.B.; Schraub, S.; Sneeuw, K.; Sullivan, M.; Takeda, F. (1993): The European Organization for Research and Treatment of Cancer QLQ-C30: a quality-of-life instrument for use in international clinical trials in oncology. Journal of the National Cancer Institute 85(5): $365-376$.

Andrews, F.M.; Withey, S.B. (1976): Social Indicators of Well-being. America's Perception of Life Quality. New York: Plenum Press.

Angermeyer, M.C.; Kilian, R. (2006): Theoretical Models of Quality of Life for Mental Disorders. In: Katschnig, H.; Freeman, H.; Sartorius, N. (Hg.): Quality of Life in Mental Disorders. Second Edition. Chicester: John Wiley \& Sons, 21-32.

Angermeyer, M.C.; Kilian, R.; Matschinger, H. (2000): WHOQOL-100 und WHOQOL-BREF. Handbuch für die deutschsprachige Version der WHO Instrumente zur Erfassung von Lebensqualität. Göttingen/Bern/Toronto/Seattle: Hogrefe Verlag.

Bayertz, K. (2010): Eine Wissenschaft vom Glück. Erster Teil: Was ist Glück? Zeitschrift für philosophische Forschung 64(3): 410-429.

1 | Zur Verteilung bzw. Nutzung des Konzepts der Lebensqualität in gesundheitsökonomischen Bewertungen siehe auch den Beitrag von Kathrin Dengler in diesem Band. 
Bollen, K.A.; Curran, P.J. (2006): Latent Curve Models. A Structural Equation Perspective. Hoboken: John Wiley \& Sons.

Boss, J. (1978): The Medical Philosophy of Francis Bacon (1561-1626). Medical Hypotheses 4(3): 208-220.

Bowling, A. (2001): Measuring Disease. Buckingham: Open University Press.

Brooks, R.; Rabin, R.; de Charro, F. (2003): The Measurement and Valuation of Health Status Using EQ-5D. A European Perspective. Evidence from the EuroQol BIO MED Research Program. New York: Springer.

Cummins, R.A. (1996): The domains of Life Satisfaction: An Attempt to Order Chaos. Social Indicators Research 38: 303-328.

Cummins, R.A. (2000): Objective and Subjective Quality of Life: An Interactive Model. Social Indicators Research 52: 55-72.

Cummins, R.A.; Gullone, E.; Lau, A.L.D. (2002): A Model of Subjective Well-being Homeostasis: The Role of Personality: In: Gullone, E.; Cummins, R.A. (Hg.): The Universality of Subjective Well-being Indicators: A Multi-disciplinary and Multi-national Perspective. New York: Springer, 7-46.

Cummins, R.A.; Nistico, H. (2002): Maintaining Life Satisfaction: The Role of Positive Cognitive Bias. Journal of Happiness Studies 3: 37-69.

Cummins, R.A. (2005): Moving from the Quality of Life Concept to a Theory. Journal of Intellectual Disability Research 49(10): 699706.

Diener, E.; Diener, C. (1996): Most People Are Happy. Psychological Science 7: 181-185.

Diener, E. (1984): Subjective Well-being. Psychological Bulletin 95(3):542-575.

Diener, E. (2000): Subjective Well-being. The Science of Happiness and a Proposal for a National Index. American Psychologist 55(1): 34-43.

Diener, E.; Emmons, R.A.; Larsen, R.J.; Griffin, S. (1985): The Satisfaction With Life Scale. Journal of Personality Assessment 49(1): 71-75. 
Elkinton, J.R. (1966): Medicine and the Quality of Life. Annals of Internal Medicine 64(3): 711-714.

Galbraith, J.K. (1964): Economics and the Quality of Life. Science 145(3628): 117-123.

Henderson, L.W.; Knight, T. (2012): Integrating the Hedonic and Eudaimonic Perspectives to More Comprehensively Understand Wellbeing and Pathways to Wellbeing. International Journal of Wellbeing 2(196): 221.

Jacobson, A.M. (1997): Quality of Life in Patients With Diabetes Mellitus. Seminars in Clinical Neuropsychiatry 2(1): 82-93.

Jacobson, A.M.; de Groot, M.; Samson, J.A. (1994): The Evaluation of Two Measures of Quality of Life in Patients with Type I and Type II Diabetes. Diabetes Care 17(4): 267-274.

Johnson, L.B. (1964): The Great Society. In: Public Papers of the Presidents of the United States, Lyndon B. Johnson. Book I (1963-64) Washington DC: Government Printing Office, 704-707.

Kilian, R.; Angermeyer, M.C. (1999): Quality of Life in Psychiatry as an Ethical Duty: From the Clinical to the Societal Perspective. Psychopathology 32: 127-134.

Kilian, R.; Dietrich, S.; Toumi, M.; Angermeyer, M.C. (2004): Quality of Life in Persons with Schizophrenia in Out-patient Treatment with First- or Second-generation Antipsychotics. Acta Psychiatrica Scandinavica 110(2): 108-118.

Kilian, R.; Lauber, C.; Kalkan, R.; Dorn, W.; Rossler, W.; Wiersma, D.; van Buschbach, J.T.; Fioritti, A.; Tomov, T.; Catty, J.; Burns, T.; Becker, T. (2012): The Relationships Between Employment, Clinical Status, and Psychiatric Hospitalisation in Patients with Schizophrenia Receiving either IPS or a Conventional Vocational Rehabilitation Programme. Social Psychiatry and Psychiatric Epidemiologie 47(9): 1381-1389.

Krupinski, J. (1980): Health and Quality of Life. Social Science and Medicine 14A: 203-211.

Langle, G.; Steinert, T.; Weiser, P.; Schepp, W.; Jaeger, S.; Pfiffner, C.; Frasch, K.; Eschweiler, G.W.; Messer, T.; Croissant, D.; Becker, T.; Kilian, R. (2012): Effects of Polypharmacy on Outcome 
in Patients with Schizophrenia in Routine Psychiatric Treatment. Acta Psychiatrica Scandinavica 125(5): 372-381.

Lehman, A.F. (1997): Instruments for Measuring Quality of Life in Mental Disorders. In: Katschnig, H.; Freeman, H.; Sartorius, N. (Hg.): Quality of Life in Mental Disorders. Chichester: John Wiley \& Sons, 79-94.

Lehman, A.F. (2006): Instruments for Measuring Quality of Life in Mental Disorders. I: up to 1996: In: Katschnig, H.; Freemann, H.; Sartorius, N. (Hg.): Quality of Life in Mental Disorders. Chicester: John Wiley \& Sons, 71-84.

More, T. (1901): New York: Ideal Commenwealth. P.F. Collier \& Son. The Colonial Press.

Neumann, J.v.; Morgenstern, O. (1953): Theory of Games and Economic Behavior. Third Edition. Princeton: Princeton University Press.

Power, M.; Harper, A.; Bullinger, M.; The WHOQOL Group (1999): The World Health Organization WHOQOL-10o: Test of the Universality of Quality of Life in 15 Different Cultural Groups Worldwide. Health Psychology 18: 495-505.

Pukrop, R. (2002): Subjektive Lebensqualität. Kritische Betrachtung eines modernen Konstrukts. Der Nervenarzt 74: 48-54.

Schöffski, O.; Glaser, P.; Schulenburg, J.M.v.d. (1998): Gesundheitsökonomische Evaluationen. Grundlagen und Standortbestimmungen. Berlin: Springer.

Schwartz, C.E.; Sprangers, M.A. (1999): Methodological Approaches for Assessing Response Shift in Longitudinal Health-related Quality-of-life Research. Social Science and Medicine 48(11): 15311548 .

Skevington, S.M. (2002): Advancing Cross-cultural Research on Quality of Life: Observations Drawn from the WHOQOL Development. Quality of Life Research 11:135-144.

Sprangers, M.A.; Schwartz, C.E. (1999): Integrating Response Shift into Health-related Quality of Life Research: A Theoretical Model. Social Science and Medicine 48(11): 1507-1515. 
Stevens, S.S. (1946): On the Theory of Scales and Measurement. Science 103: 677-680.

Szende, A.; Oppe, M.; Devlin, N. (2007): EQ-5D value sets. Inventory, Comparative review and User Guide. Dordrecht: Springer.

The WHOQOL Group (1998a): Development of the World Health Organization WHOQOL-BREF Quality of Life Assessment. Psychological Medicine 28: 551-558.

The WHOQOL Group (1998b): The World Health Organization Quality of Life Assessment (WHOQOL): Development and General Psychometric Properties. Social Science and Medicine 46: $1569-1585$.

Ware, J.E.; Gandek, B.; The IQOLA Project Group (1994): The SF36 Health Survey: Development and Use on Mental Health Research and the IQOLA Project. International Journal of Mental Health 23: 49-73.

Ware, J.E. Jr. (1984): Methodology in Behavioral and Psychosocial Cancer Research. Conceptualizing Disease Impact and Treatment Outcomes. Cancer 53(10 Suppl): 2316-2326.

Ware, J.E. Jr. (1991): Conceptualizing and Measuring Generic Health Outcomes. Cancer 67(3 Suppl): 774-779.

Ware, J.E. Jr. (2003): Conceptualization and Measurement of Healthrelated Quality of Life: Comments on an Evolving Field. Archives of Physical Medicine and Rehabilitation 84(4 Suppl 2): 43-51.

Wilson, I.B. (1999): Clinical understanding and clinical implications of response shift. Social Science and Medicine 48(11): 1577-1588.

World Health Organization (2001): Internationale Klassifikation der Funktionsfähigkeit, Behinderung und Gesundheit (ICF). Deutsche Ausgabe 2005. Genf: Deutsches Institut für Medizinische Dokumentation und Information. 


\section{Wert und Werthaltungsbegründung}

Zur Vereinbarkeit von moralischen, ökonomischen, rechtlichen und medizinischen Werten

Günter Fröhlich

Die Rede von Werten ist weitgehend heterogen. Je nach Sachzusammenhang verstehen wir darunter offenbar etwas Verschiedenes. In der Frage nach Zuteilungskriterien im Gesundheitssystem wollen wir aber die unterschiedlichen Bewertungsfaktoren medizinisch, rechtlich, ökonomisch und moralisch aufeinander beziehen. Es macht daher Sinn, zuerst danach zu fragen, was Werte überhaupt sind, oder wie solche innerhalb der verschiedenen Bereiche der Begriffsverwendung verstanden werden, um im Anschluss daran das Problem der Vielfalt ethischer Begründungstypen zu beleuchten, die sich in der philosophischen Tradition herausgebildet haben, sich aber auch - unterschiedlich gesättigt - in Alltagswerthaltungen oder in politischen Konzeptionen auffinden lassen. Ein solches Vorgehen impliziert die Behauptung, dass sich letztlich auch medizinische, rechtliche oder ökonomische Bewertungen ethisch ausweisen lassen müssen. Im anderen Fall könnten wir aber gar keine Vergleichskriterien feststellen, welche es ermöglichen, die Bewertungssachlagen aufeinander zu beziehen.

Dass wir Zustände, Vorgehensweisen, Zuteilungskriterien usf. überhaupt bewerten, liegt, so die These dieses Beitrages, an Werthaltungen von Personen. In Bezug auf das Gesundheitssystem besteht das ethisch entscheidende Kriterium im Wohl und Willen des Patienten sowie in seiner wohlverstandenen, d.h. durch ihn selbst 
definierten und bewerteten Lebensqualität. Es handelt sich dabei um Wertorientierungen, die offensichtlich in den derzeitigen Diskussionen im Gesundheitswesen keine Rolle spielen, weil diese von medizinischen, rechtlichen und vor allem ökonomischen Bewertungen dominiert werden. Zwar führen die an der Diskussion Beteiligten (z.B. Vertreter der politischen Parteien, von Ärzteverbänden, von Versicherungen) die Patientenselbstbestimmung sowie dessen Wohl ständig im Munde, tatsächlich aber findet der Patient in der politischen Auseinandersetzung über die medizinische Versorgung keine ausreichende Berücksichtigung, ${ }^{1}$ und das, obwohl die Rechtslage durch die Selbstbestimmungsrechte des Grundgesetzes und die entsprechenden richterlichen Entscheidungen dies eindeutig fordern (vgl. Fröhlich 2012a). Zugegebenermaßen mag in der einzelnen Beziehung von Arzt und Patient eine Orientierung an den Bedürfnissen und Wünschen des Patienten mitunter einmal vorkommen; das ist dann aber eher zufällig bzw. findet statt, weil die Behandelnden sich einem traditionellen Fürsorgeethos verpflichtet fühlen, das unter modernen Gesichtspunkten zuweilen auch die Wünsche des Patienten berücksichtigt, um so einem zu einseitigen Paternalismus zu entgehen, der das vermeintliche Wohl des Patienten über seinen Willen stellt.

\section{Die Frage nach dem Wert}

Was ein »Wert« ist, kann niemand exakt sagen (vgl. Schönrich 2008, S. 97; vgl. Kutschera 2010, S. 11ff.; vgl. Tappolet 2009, S. 459; vgl. für das Folgende auch Fröhlich 2011, S. 17f., 21ff.). Die ontologische Bestimmung davon, was Werte sind, wird immer umstritten

1 Der Patient erscheint sozusagen nur noch als Empfänger von Gesundheitsleistungen. Zwar gibt es in den einschlägigen Gremien durchaus Patientenvertreter, diese haben aber eine äußerst schwache Stellung. Die Interessen der beteiligten Parteien sind in den allermeisten Fällen mit den Interessen der Patienten unvereinbar. 
bleiben. Die Rede von Werten ist zudem auch noch extrem kontrovers. Offenbar aber ist, dass, um von Werten zu sprechen, ein Subjekt vorausgesetzt werden muss (vgl. Hügli et al. 2004, S. 557, 599ff.; vgl. Kuhn 1975, S. 345). Das heißt aber nicht, dass das, was einen Wert ausmacht, nur davon abhängt, was das Subjekt sich darunter vorstellt oder konstruiert, also sozusagen ohne objektive Außenhalte. Vielmehr sind mit Subjekten nur die Bedingungen gesetzt, bestimmte Aspekte der Welt werthaft zu betrachten, um dann »Werte« in den Umgebungen menschlichen Handelns auch zu entdecken. Damit würde sich die Rede von Werten auf bestimmte Eigenschaften von Objekten richten, die selbst in einem bestimmten Sinn für objektiv gehalten werden können. ${ }^{2}$

Nun sind so genannte Subjekte selbst schon komplexe Gebilde, die einen Willen und Bedürfnisse haben bzw. die zum Handeln getrieben werden. Deswegen entwerfen Subjekte Ziel- und Zwecksetzungen, die sie in höherwertige oder niedrigerwertige einteilen. Für die Neuzeit stammt der Begriff des Werts aus der Ökonomie. Adam Smith bestimmte einen Tauschwert, wenn der Besitz eines Guts gegen den Besitz eines anderen Guts eingetauscht werden kann, und einen Gebrauchswert, der in der Nützlichkeit einer Sache liegt (vgl. Smith 2001, S. 27). Immanuel Kant nimmt einen absoluten Wert an, der nicht, wie der Preis, austauschbar ist und Hermann Lotze (im Übrigen ein Arzt) setzt im 19. Jahrhundert bereits ein Gefühl für Werte an (vgl. Lotze 1896, S. 275 sowie Lotze 1905, S. 314). Lotze meinte aber auch, dass Werte nicht nur danach beurteilt werden können, wie diese in uns entstehen, sondern dass wir

2| Hermann Lotze bindet den "Wert" fast vollständig an das Fühlen von Subjekten, obwohl er umgekehrt versucht, eine "allgemeine Gültigkeit der Wertungen zu sichern“ (Hügli et al. 2004, S. 559). Ähnlich argumentiert heute Franz von Kutschera, nach dem wir von der "Objektivität von Werttatsachen “ nicht absehen können. In Frage steht die Deutung dieses Befunds: Sind Werte nur subjektive Präferenzen oder kommt innen selbst Objektivität zu (vgl. Kutschera 2010, S. 19f.)? Zum Objektivitätscharakter von Werten vgl. auch Harth 2008, S. 24. 
auch fragen müssen, was sie sind (vgl. Lotze 1912, S. 612, 615). Das ist weiterhin für ihn eine moralphilosophische Frage. Unter diesen Voraussetzungen hat sich der Begriff des Werts weit verzweigt.

Nach Franz von Kutschera müssen wir zuerst zwischen »klassifikatorischen« (wie nützlich - nutzlos), »komparativen« (wertvoller oder ebenso wertvoll) und »metrischen« (die in Zahlen ausgedrückten Nutzwerte) Wertbegriffen unterscheiden. Daneben gibt es Werte für verschiedene Bereiche wie Ethik, Ästhetik, Sport oder Wissenschaft. Werte, wie z.B. »gut«, können sich als Prädikate aber auch auf einen rein deskriptiven Sinn beziehen: Indem wir z.B. von einer Sache sagen, diese sei gut, meinen wir, dass sie die Bedingungen, die wir von ihr erwarten, erfüllt. Ein gutes Messer ist eines, das schneidet, sonst können wir es nicht seinem Gebrauch gemäß benutzen. Dass ein gutes Messer einen Funktionswert hat und dass ein gutes Messer scharf ist, sind unterschiedliche Aspekte. Allerdings können sich solche deskriptiven Prädikate mit wertenden mischen. Das ist bei moralischen oder ästhetischen Aussagen häufig der Fall (vgl. Kutschera 2010, S. 12-15).

Nach Helmut Kuhn sieht man dem Wertbegriff »seine Herkunft aus dem Bereich der Wirtschaft« (Kuhn 1975, S. 343) an. Im Grunde rezipiert die Wertphilosophie im ausgehenden 19. Jahrhundert aber schon Traditionen aus der Antike (vgl. Galewicz 1990). Auch wenn mit der Ökonomie ein bestimmter Wertbegriff aufkommt (vgl. Hügli et al. 2004, S. 556; Lichtblau 2004, S. 586), sind die meisten Begriffe der Ökonomie philosophischen Ursprungs (vgl. Vossenkuhl 2006, S. 30of.; vgl. Hemel 2007). Schon Aristoteles diskutiert in seiner Nikomachischen Ethik Preisbindung und gerechten Tausch (vgl. Aristoteles NE V, 8). Auch dem Begriff des ökonomischen Tauschs liegt eine Theorie der Gerechtigkeit zugrunde, nach der es notwendig wird, das »Selbstverständnis des Lebens überhaupt« (Kuhn 1975, S. 350) zu klären und eine allgemeine Theorie des Guten zu entwickeln (vgl. ebd., S. 354-358). Der ökonomische Wert als Preisäquivalent ist ein Spezialfall allgemeiner philosophischer Begriffsbildungen. Ich nehme mit Peter Ulrich an, dass es keine 
»wertfreie ökonomische Theorie« geben kann. ${ }^{3}$ Dann besteht mit Max Weber aber die Verpflichtung einer Wissenschaft, ihre Werthaltungen zu explizieren (vgl. Weber 1968). Das gilt für das Recht genauso wie für die Medizin, in den Grundlagen wie in den Anwendungen.

Eine weitere Differenzierung ergibt sich, wenn wir fragen, was wir bewerten: die Dinge, die Ziele (die antizipierten oder die tatsächlichen Folgen), die Mittel, ${ }^{4}$ die Prinzipien, den Willen, die zentralen Motivationen, die Haltungen, die Begründungen der Handlung, das Verfahren zum Handlungsentwurf oder allgemein die Werthaltungen. ${ }^{5}$ Dadurch und durch die möglichen Vermischungen von Wertprädikaten in ihren Klassen der Moral, der Medizin, des Rechts und der Ökonomie sowie in ihren deskriptiven und normativen Dimensionen wird jede Einzelfallentscheidung im klinischen Kontext auch immer moralisch hoch komplex. Neue therapeutische Möglichkeiten, v.a. zur Lebensverlängerung, werden also nicht nur in technisch-medizinischer Sicht immer komplizierter, sondern sie bedingen gleichermaßen neue Unübersichtlichkeiten der ethischen Bewertung. Dies ist nicht zuletzt deshalb der Fall, weil auch die Moral ihre Entwicklung hat, die sich jeweils in den Handlungssubjektiven absättigen muss, was dazu führt, dass unsere moralischen Intuitionen dem rasanten technischen Fortschritt hinterher hinken.

3 | Nach Peter Ulrich enthält die Ökonomie ein normatives Programm, das sich "aspektbezogen als allgemeine Theorie menschlichen Verhaltens sowie der gesellschaftlichen Institutionen schlechthin" (Ulrich 2002, S. 1) versteht. Der Grundwert der heutigen Wirtschaftstheorie liegt nach Ulrich im Prinzip der Gewinnmaximierung.

4 | Vgl. zum "Ethos der Mittel“ Fröhlich und Rogler 2005, S. $7 f$.

5 | Manfred Harth z.B. fasst Werte als "Informationen “ auf: „Ethische Wahrheit ist stabile Rechtfertigbarkeit vor dem Hintergrund bestimmter invariabler ethischer Informationen, d.h. bestimmter ethischer Prinzipien, Wertvorstellungen oder moralischer Empfindungen, die eine Person, Gemeinschaft oder Kultur zu einem bestimmten Zeitpunkt vertritt bzw. hat" (Harth 2008, S. 169f.; vgl. auch ebd., S. 171, 177ff., 205ff.). 
Ethik als »reflexives Nachdenken über das Gute« (vgl. Fröhlich 2006) hat freilich eine Orientierungsfunktion zu leisten, die komplexitätsreduzierend für das Handeln sein soll. ${ }^{6}$ Dieser Umstand kann nicht gegen die Ethik verwendet werden, indem gesagt wird, dass ethische Überlegungen über das richtige Handeln die Entscheidungsspielräume so verengen, dass situationsbezogen gar nicht mehr geurteilt werden könne. ${ }^{7}$ Wir können also nicht beides haben, eine begründete und bestimmende Orientierung, die uns Handlungsregeln für umgrenzte Fallsituationen angibt, und die totale Handlungsoffenheit, die sich zuletzt bei Einzelfallentscheidungen nicht mehr rechtfertigen lassen wird. Die Anwendung von ethischen Maßstäben schränkt die Optionen des Handelns zwangsläufig ein, das ist aber gerade ihr Sinn. Wie diese inhaltlich aussehen, ist damit aber noch nicht festgelegt.

Eine letzte, auf Aristoteles zurückgehende Unterscheidung kann uns ein weiteres Problem erhellen: »Ethik « ist per definitionem Nachdenken des einzelnen über seine Handlungen (vgl. Fröhlich 2006). Den öffentlichen Sektor bezeichnet Aristoteles als »Politik«. Der Begriff ist erheblich weiter zu verstehen, als wir das heute üblicherweise tun. Die Klammer zwischen den beiden Handlungsarten ist aber wieder der einzelne, nur der Bezug der betroffenen Situationsumstände, auf Privates (»idiotisch«) oder Öffentliches (»politisch«) ist ein anderer. Auch öffentliches Handeln vollzieht sich in Handlungen von Einzelsubjekten. Ebenso sind von solchen Entscheidungen nicht »Gruppen « betroffen, sondern wiederum immer

6 | Das hat auf begrifflicher Ebene natürlich zur Folge, dass die Reflexion über das Gute höhere Komplexitätsgrade annimmt. Die Vermittlung zwischen den komplexen begründungstheoretischen Strukturen der Ethik und der Alltagswelt ist umgekehrt ebenso eine Aufgabe der Ethik.

7 | Dies geschieht implizit teilweise zum Beispiel in der Kritik an Bewertungsinstrumenten zur Evaluation von Gesundheitsleistungen, wenn gesagt wird, dass sie dem Einzelfall nicht genügen. Vgl. auch die Dimension $10 \mathrm{im}$ Beitrag von Dengler und Fangerau zu den Prämissen von gesundheitsökonomischen Bewertungsverfahren. 
konkrete und einzelne Personen. Dass wir diese in beliebiger Weise zu Gruppen, Kohorten, Klassen oder was sonst zusammenfassen können, um statistische Evaluationen zu gewinnen oder Fallpauschalen zu generieren, ändert daran naheliegenderweise nichts. ${ }^{8}$ Jede therapeutische Intervention richtet sich zuletzt immer auf den einzelnen, betroffenen Patienten.

Der Begriff des Werts, als Objekt gemeinsam gefällter Werturteile (auf derselben Ebene!), überbrückt, wie ich behaupten möchte, dieses Bezugsproblem des Öffentlichen - und das unabhängig davon, wie der einzelne sein Werturteil begründet. ${ }^{9}$ Die Zielorientierung öffentlichen Handelns von einzelnen Individuen, sei es auf das größtmögliche Wohlergehen, auf Rechtssicherheit, auf den Standard der Medizin lege artis, auf das Klima im Team oder den sozialen Frieden, auf Gerechtigkeit, darauf, jedem, das ihm Zukommende zu gewähren, auf das Arzt-Patienten-Verhältnis usf., beruht vollständig auf solchen gemeinsamen Werthaltungen, die freilich faktisch durch das Moment der politischen Durchsetzungsfähigkeit in ihrer inhaltlichen Ausrichtung verschoben werden können. ${ }^{10}$ Das lässt sich immer dann beobachten, wenn auch nach langer und abwägender, öffentlicher Diskussion Maßnahmen beschlossen werden, welche zuletzt nicht die erhoffte Wirkung zeigen. Sollte sich die zunehmende, zahlenbasiert-ökonomische Ausrichtung im Gesundheitswesen als inadäquat für die sachliche Einholung der Patientenbedürfnisse erweisen lassen, wird die ausschließliche An-

8| Mit dieser Kritik sei ausdrücklich auch nichts über den Sinn solcher Zusammenstellungen gesagt.

9 | Das setzt freilich voraus, dass der einzelne sich auch ein Werturteil bildet, und dass er das als solches expliziert. Eine auf diesem Umstand aufbauende Theorie der Ethikberatung im klinischen Kontext wird derzeit vom Verfasser entwickelt und soll 2013 erscheinen. Einige Grundüberlegungen hierfür finden sich in Fröhlich 2012c.

10 | Carmen Kaminski will dagegen in pragmatischen Diskursen festlegen, was öffentlich zu gelten hat; die oberste Norm ist dabei die "politische Durchsetzbarkeit» (vgl. Kaminski 2005; vgl. hierzu auch Fröhlich 2009). 
wendung ökonomischer Methoden zwangsläufig solche Fehlsteuerungen generieren.

Eine Rekonstruktion der unterschiedenen Wertzuordnungen und die klare Differenzierung ihres jeweiligen Sachgehalts ist die notwendige Voraussetzung, die verschiedenen relevanten Werte und die vorgebrachten Werthaltungen in einer Entscheidungssituation aufeinander $\mathrm{zu}$ beziehen und $\mathrm{zu}$ gewichten, um daraus ein begründetes Urteil abzuleiten. Die Handlungsorientierung ist damit noch nicht vorgeprägt, denn angesichts der Komplexität von Wertverteilungen ist es jederzeit möglich, in Bezug auf die Situation, die Ziele oder die zu ergreifenden Mittel diese umzugewichten, um dadurch zu neuen Handlungsalternativen zu kommen. Jede Differenzierung auf einer solchen Grundlage wird immer sehr schnell einsichtig machen, auf welcher Ebene und wie Werte miteinander konfligieren. Dem Verfahren liegt letztlich noch ein Retorsionsproblem zugrunde, indem die Gewichtung der Handlungsoptionen freilich wieder auf Werthaltungen fundiert ist. Diese werden aber erst aufgrund einer Evaluation und einer Analyse der Fundierungsund Konsequenzzusammenhänge der unterschiedlichen beteiligten Werthaltungstypen sichtbar. Nach der Analyse können diese dann allerdings als solche expliziert und diskutiert werden, woraus sich in der Folge auch neue Lösungswege, die für alle Beteiligten und Betroffenen gangbar sind, öffnen dürften.

\section{Werthaltungen Und ethische BEGRÜNDUNGSTYPEN}

Nach einer klassischen Einteilung werden Wertbegründungstypen vielfach nach einer tugendbasierten, einer deontologischen und einer konsequentialistischen Ethik differenziert. ${ }^{11}$ So sinnvoll eine

11 | Vgl. zum Beispiel auch die von Dengler und Fangerau in ihrem Beitrag zu den Prämissen gesundheitsökonomischer Bewertungsverfahren beschriebene Dimension 10 "Einengung von Entscheidungsspielräumen", 
solche Klassifizierung der Übersicht halber ist, und so sehr diese in der Literatur und in den Bewertungsmaßstäben zur Zuteilung von Gesundheitsleistungen rezipiert wird, so bestehen dabei nicht unerhebliche, und zum Teil auch ungesehene Probleme, die vielfach $\mathrm{zu}$ Missverständnissen und Schwierigkeiten führen. ${ }^{12}$

Es scheint dabei durchaus korrekt zu sein - wie der Beitrag von Dengler und Fangerau in diesem Band belegt -, dass die derzeit zugrunde gelegten Maßstäbe der Gesundheitsleistungszuteilung aus

wenn hier in kritischer Hinsicht betont wird, dass konsequentialistische Ansätze unzureichend oder gar nicht auf Individuen oder konkret gegebene Situationen bezogen werden können.

12 | Das Argument, die Philosophie könne in Diskursen der öffentlichen Moral nicht mitreden, weil den Philosophen sowohl die Kompetenz für die einzelnen Bereiche als auch für die Komplexität der Sachlagen abgeht (vgl. Kaminski 2005), ist meines Erachtens nicht stichhaltig (vgl. Fröhlich 2009). Wenn es um moralische Orientierung geht und dahingehend um eine ethische Expertise, so gehört das freilich in den Bereich der Philosophie. Dabei ist das Vorurteil abzulegen, eine ethische Analyse würde zweifelsfrei feststellen, worin das richtige Handeln liegt, die Vorstellung also, dass durch die Moralphilosophie Lösungen generiert werden, an die der Handelnde sich zu halten hat. Wichtig ist in diesem Zusammenhang auch der Umstand, dass Werthaltungen, auch wenn diese öffentlichkeitswirksam werden, nicht einfach der modernen Welt entspringen. Diese gehen vielmehr aus der philosophischen Tradition und der allgemeinen Ideengeschichte hervor, und schlagen sich dann in unterschiedlichen Verzweigungen in den Werthaltungen der öffentlichen Akteure und ihren politischen Beobachtern (Bürger, Journalisten, Wissenschaftler) nieder. Trotz der öffentlichen Relevanz der Entscheidungen werden solche von einzelnen Personen getroffen und zum Teil auch verantwortet. Die Komplexität der modernen Institutionen spielt freilich für die Personenverhältnisse und der in diesen vernetzten Akteuren eine Rolle, sodass weitreichende öffentlich wirksame Entscheidungen sicher nicht allein von Einzelpersonen getroffen werden. Aber dieses Problem entsteht schon auf der Ebene relativ überschaubarer Teams wie z.B. auf einer klinischen Station. 
einem bestimmten Typ konsequentialistischer Spielart resultieren, die sich unter einer ökonomischen Rigide zusätzlich verkürzt, indem fast ausschließlich monetäre Werte berücksichtigt werden. ${ }^{13}$ Dabei enthält die Fokussierung des Beitrags zu den Prämissen gesundheitsökonomischer Bewertungssysteme bei der Ermittlung der Bewertungsdimensionen, was unser Problem angeht, drei Beurteilungstendenzen: Erstens, dass diese konsequentialistisch-monetäre Einseitigkeit nicht durch deontologische Programme, sondern durch tugendethische Ansätze im Anschluss an Aristoteles zu beheben sind; zweitens, dass eine individualethische Ausrichtung, vor allem am Arzt-Patienten-Verhältnis, die rein monetär basierte Folgenabschätzung von Zuteilungsprinzipien am Maßstab der Betrachtung des Gesundheitssystems, von den Durchschnittskosten und der Verteilung bestimmter Indikationen innerhalb der Bevölkerung verhindern würde; und drittens, dass einzelne Werte durch verschiedene Begründungstypen fundiert werden müssen, also z.B. die Selbstbestimmung des Patienten durch die deontologische Ethik oder das Arzt-Patienten-Verhältnis durch den tugendethischen Ansatz.

Strategisch (das heißt ja der Einfachheit halber) soll hier so vorgegangen werden, dass im Rekurs auf die drei moralbegründungstheoretischen Tendenzen der konsequentialistische Ansatz verteidigt, der deontologische in sein Recht und der tugendethische in seinen problematischen Ausrichtungen pointiert werden soll. Das soll einen Ausgleich schaffen, der auf den Ausführungen im zitierten Beitrag zu den Prämissen gesundheitsökonomischer Bewertungssysteme dahingehend basiert, als dessen Einschätzungen

13 | Dabei werden offenbar auch außermonetäre Werte entweder wieder auf eine monetäre Basis oder wenigstens auf ein quantitatives Verhältnis gebracht, was angesichts der mathematischen Methoden, welche erst eine Berechnung und damit einen direkten Vergleich durch Korrelationen der Bezugsgrößen erlauben, nicht verwunderlich ist (vgl. zu den Grundlagen von Korrelationen von definierten Zielbereichen bei mathematischen Funktionen Fröhlich 2012b). 
durchweg nachvollziehbar sind. Dennoch wird dadurch stellenweise nur eine sehr verkürzte Lesart der wertbasierten Orientierungen traditioneller Ethikbegründungen geboten. Ich will mich hier allerdings auf Grundsätzliches beschränken, vor allem weil die pointierte Darstellung der Ausräumung von Vorurteilen besser dient als eine sehr viel weitergehende philosophische Analyse und Interpretation der jeweiligen Textgrundlagen.

\subsection{Der Utilitarismus}

Die zwingende Gleichsetzung von Folgeabschätzungen mit monetären Bewertungen ist trivialerweise falsch für den Utilitarismus. Allerdings hat Jeremy Bentham mit seinem Vorschlag zum Glückskalkül, der Auffassung also, dass sich die Glücksmenge berechnen lässt, eine solche Ausrichtung gewiss vorbereitet, und die neuen Möglichkeiten zur mathematischen Formalisierung haben den Bereich für Berechnungen erheblich ausgedehnt. Hinzu kommt die Verkürzung von Glück auf die Befriedigung von Interessen und Bedürfnissen im Utilitarismus. John Stuart Mill, meines Erachtens einer der maßgeblichen Vertreter, hat aber immer betont, dass es in erster Linie um die Interessen der Gemeinschaft geht (vgl. Fröhlich 2010, S. 237, 240, 245ff., 256f.). Und er nahm an, dass der Mensch verschiedene Bedürfnisse hat, und eben ausdrücklich auch geistige (vgl. ebd., S. 241, 247ff., 263). ${ }^{14}$

Ein anderer Punkt liegt in der Frage nach den Prinzipien im Utilitarismus und in der Bestimmung des Verhältnisses von Individuum und Gemeinschaft. Es bestehen die Vorurteile, dass im Utilitarismus keine moralischen Grundsätze gelten können, und dass ein Individuum, sollte es der Gemeinschaft nützen, selbstverständlich geopfert werden dürfe. Mill leitet dagegen eine Reihe von Prinzipien aus der allgemeinen Erfahrung ab, an die wir uns halten

14 | Eine anregende Diskussion um diese Probleme unterschiedlicher Qualitäten von Lust bei Mill findet sich bei Henry Sidgwick 1909 im ersten Band S. 46ff., 96ff., 109, 180 sowie im zweiten Band S. 274ff. 
sollten, weil diese sich bewährt haben (vgl. Fröhlich 2010, S. 243, 246, 261f.) - eine ähnliche Position finden wir z.B. im Rechtspositivismus bei der Begründung der Menschenwürde. Mill ist freilich auch ein Regelutilitarist, gehört also der Richtung des Utilitarismus an, die annimmt, dass es erfahrungsbasierte Prinzipien gibt, deren Beachtung das Gemeinwohl besser fördern als ihre Aussetzung in vermeintlich anders gelagerten Einzelfällen (vgl. ebd., S. 232f., sowie 237, 243).

Was die individuellen Rechte angeht, so plädiert Mill eindeutig dafür, diese zu schützen, wenn auch aus Nützlichkeitsgründen (vgl. ebd., S. 257f.). Gesetze, allgemeine Regeln, tradierte Verhaltensweisen und gesellschaftliche Umgangsformen geben uns Sicherheit. Wenn wir ständig bedroht sind, belogen und betrogen zu werden, wenn auch nur im Einzelfall und wohlbegründet durch den allgemein gesellschaftlichen Vorteil, können wir nicht zufrieden leben. Deswegen gelten solche Prinzipien auch für Mill absolut. Ihre Untergrabung kann auf keinen Fall das allgemeine Wohl befördern. Wer z.B. einen Freund belügt, lädt Schuld auf sich, auch wenn dadurch viele einen Vorteil gehabt haben sollten. Es gibt eine Tendenz bei Mill dazu, dass sich der moralisch Handelnde für die Gemeinschaft opfern sollte (vgl. ebd., S. 238f.), aber das muss er auch bei ihm immer selbst entscheiden können. Die Autonomie der Entscheidung für das eigene Wohl und Wehe kann also nicht durch einen Mehrheitsentscheid der übrigen Mitglieder der Gesellschaft ersetzt werden.

\subsection{Die deontologische Ethik}

Der Pflichtenethik wird vorgeworfen, dass ihr Ansatz erstens rigoros und insofern nicht mit anderen Positionen vermittelbar ist, und - zweitens - kaum auf Einzelfallentscheidungen angewendet werden kann, da die Prinzipienfundierung kein Augenmerk auf die konkrete Handlungssituationen richtet. Allenfalls wenn es um absolute Begründungen wie die Menschenrechte oder die Selbstbestimmung des Menschen (Autonomie) geht, behalte diese ihr 
relatives Recht. Es sieht dann so aus, als ob alternative Ansätze generell nicht in der Lage sind, solche Prinzipien zu begründen, oder allenfalls zu schwach, sodass diese im Einzelfall immer zur Disposition stehen. Umgekehrt scheint die Deontologie zwar die wichtigsten Grundprinzipien unserer moralischen Orientierung sicher begründen zu können, die Anwendung der Prinzipien dagegen ist anscheinend unmöglich, weil der absolute Wert nie auf die konkrete Handlungssituation passen kann.

Nun ist speziell die Kantische Moral als eine Maximenethik entworfen. Maximen sind bekanntlich subjektive Grundsätze. ${ }^{15}$ Kant unterscheidet objektive, praktische Gesetze und eben subjektive Regeln (Maximen), die nur für das Subjekt gültig sind. Solche Maximen gehen aus einer Vermittlung zwischen den Bedürfnissen des Subjekts und seiner Handlungssituation hervor. Sie stehen aber unter dem praktischen Gesetz, das die Vernünftigkeit des subjektiven Grundsatzes fordert. Die Vernünftigkeit besteht in zwei formalen Voraussetzungen: Der Handlungsgrundsatz muss widerspruchsfrei sein, das heißt, es dürfen in ihm keine Bedingungen stecken, welche in contradictio zueinander stehen, und das Subjekt muss die Folgen, welche sich aus der allgemeinen Anwendung des

15 | So heißt es schon in der Kritik der reinen Vernunft: „Praktische Gesetze, so fern sie zugleich subjektive Gründe der Handlungen, d.i. subjektive Grundsätze werden, heißen Maximen. Die Beurteilung der Sittlichkeit, ihrer Reinigkeit und Folgen nach, geschieht nach Ideen, die Befolgung ihrer Gesetze nach Maximen" (Kant KrV B840/A 812); und der locus classicus in der Kritik der praktischen Vernunft lautet freilich: "Praktische Grundsätze sind Sätze, welche eine allgemeine Bestimmung des Willens enthalten, die mehrere praktische Regeln unter sich hat. Sie sind subjektiv, oder Maximen, wenn die Bedingung nur als für den Willen des Subjekts gültig von inm angesehen wird; objektiv aber, oder praktische Gesetze, wenn jene als objektiv, d.i. für den Willen jedes vernünftigen Wesens gültig erkannt wird " (Kant KpV A 35, vgl. auch A 52). Vgl. auch Fröhlich 2011, S. 39, Fußnote 20, S. 56ff., $74 \mathrm{ff}$. 
Grundsatzes ergeben würden, auch wollen können (vgl. Fröhlich 2011, S. 60, 69, 77). ${ }^{16}$

Die Folgen von Handlungen werden also vom Kantischen Gesetz thematisiert, auch wenn es mögliche Folgen sind, denn tatsächliche Folgen können wir im Handeln gar nicht anzielen, allenfalls »verhoffte« (vgl. Kant GMS AB 13f.), da wir alle Bedingungen der Folgen und performativ dann diese auch selbst durch unser Handeln gar nicht in der Hand haben. Was Kant vom Moralischen ausschließt, ist nur, dass wir uns in unserem Willen von den inhaltlichen Vorzügen der Folgen motivieren lassen. Moralisch ist nach Kant nur, was den Willen autonom bestimmt und damit nicht heteronom durch Interessen, welche aus unserem Sinnen- und Begehrungsvermögen kausal resultieren. Das heißt aber nicht, dass wir in den luftleeren Raum hinein handeln. Ein Prinzip wie die Menschenwürde ist deswegen so allgemein verbindlich, weil sich keine Bedingungen denken lassen, unter denen diese nicht greift. Faktisch kann die Menschenwürde freilich verletzt werden, aber das ist dann unter allen denkbaren Umständen eben eine Verletzung. Was Kant im Gegensatz zum Utilitarismus für die Moral nicht interessiert, sind die tatsächlichen und kontingenten Folgen, ob diese nun das Wohl befördern oder nicht. Umgekehrt haben wir nach Kant ein Bedürfnis danach, unser eigenes Wohl und das der ganzen Menschheit zu befördern. Dieses subjektive Interesse lässt sich aber auch bei Kant dahingehend objektivieren, als es vernünftig ist, sich um sich selbst wie um die anderen zu kümmern. Die Kantische Moral schließt nur die unmittelbare Orientierung an den Interessen aus, weil die-

16 | Die zweite Bedingung wird häufig dadurch konterkariert, dass die Maxime, z.B. sich auf einen bestimmten Stuhl zu setzen, nicht gefasst werden kann, weil ich annehmen muss, bzw. sich aus dem Universalisierungsgebot der Vernunft ergibt, dass sich alle anderen dann auch auf den Stuhl setzen mögen. Das ist freilich von vorne herein schon keine Entscheidung moralischer Relevanz. Unabhängig davon werde ich wohl, wenn tatsächlich alle den Wunsch hätten, sich auf einen bestimmten Stuhl zu setzen, von meinem Wunsch absehen. 
se kontingent sind, und sich allenfalls ihre Ansprüche, nicht aber ihre konkreten Inhalte, universalisieren lassen. Das schließt aber die Berücksichtigung von Handlungsfolgen und von den Interessen der Betroffenen nicht vollständig von der Handlungsorientierung aus. Die Grundfrage für Kant ist eine der Begründung, nicht der sinnlich vermittelten Inhalte.

\subsection{Deontologie und Utilitarismus}

Wir müssen, um die Positionen der Deontologie und des Utilitarismus miteinander in Beziehung zu setzen, unterschiedliche Blickwinkel auf das Konsequenzproblem einnehmen: Der Regelutilitarismus setzt seinen Fokus auf die tatsächlichen Folgen, er meint aber ausdrücklich alle Folgen. Das willkürliche Opfern einzelner $\mathrm{zu}$ Gunsten des Gemeinwohls ist keine sinnvolle Option, weil die damit verbundene Bedrohung jedes einzelnen, auch irgendwann zu den Opfern zu gehören, das generelle Wohl niemals fördern kann. Deswegen ist es zweckmäßig, Minderheiten zu schützen und Grundrechte zu definieren. Der Utilitarist, der diese Orientierung von Mill teilt, ist sich wohl bewusst, dass die Freiheit des Handelns auf Bedingungen aufruht, die erst etabliert werden müssen. Um alle Individuen ins gleiche Recht zu setzen, fordert der Utilitarist sozialen Ausgleich, allgemein garantierte Bildung, gleichen $\mathrm{Zu-}$ gang zu Gesundheitsleistungen usf. Die durchschnittliche Hebung des Wohlstandes um jeden Preis - die ebenso gut einigen wenigen überproportional zugute kommen kann (im Gegensatz zum ökonomistischen Grundsatz, dass von der durchschnittlichen Hebung des Wohlstandes zwangsläufig alle profitieren werden, was als Kausalsatz sicher falsch ist und als empirische Behauptung zwar richtig sein kann, sich aber nicht ergeben muss) - gehört nicht zum utilitaristischen Grundprogramm. Die Regeln, welche wir befolgen, und die freilich unser Handeln einschränken, sind aber für Mill nicht absolut zu begründen, sondern eben nur aus ihren Folgen. Was da richtig und falsch ist, ist eine Sache der Erfahrung und der Tradition. 
Für Kant und die deontologische Moral dagegen ist auf die intendierten Folgen zu achten, ob diese konsistent sind. Die tatsächlichen Folgen haben wir nicht in der Hand, weil die Wirklichkeit kontingent ist. Um uns nicht von den zufälligen Erfahrungen abhängig zu machen, können wir mit Hilfe der Vernunft Handlungsprinzipien entwerfen, welche nicht aus den Inhalten unserer Interessen folgen, sondern durch die darin enthaltenen allgemein vermittelten Ansprüche begründet werden. ${ }^{17}$ Diese beruhen freilich auch wieder auf Erfahrung, weil ich sonst nicht wissen kann, was ich mir wünsche und was passieren würde, wenn sich alle Beteiligten bestimmte Grundsätze vornehmen. Die Vernunft bringt also nichts Neues in der Moral hervor, sie verallgemeinert nur. Die Rolle der Vernunft und ihr Verfahren zur Bestimmung von Verpflichtungsgründen müsste freilich noch intensiver diskutiert werden.

Im Utilitarismus sind die Regeln unsicher, die Folgen geben das Maß; in der Deontologie sind die Folgen unsicher, also geben die Regeln das Maß für das gute Handeln. Was wir tatsächlich tun sollen, muss also, ob wir es utilitaristisch oder deontologisch begründen, inhaltlich gar nicht verschieden sein - und ist es faktisch auch zumeist nicht, weil wir uns alle auf die gleiche Welt beziehen (wenigstens im Idealfall). Der Unterschied zwischen den beiden Positionen findet sich also allein darin, wie die Handlung oder ihr Prinzip rational-ethisch begründet werden oder in der Frage, wie wir zu Handlungsgrundsätzen kommen. Die Handlungsausrichtung wird aber in den meisten Fällen die gleiche sein, auch wenn zugegeben werden muss, dass es in einzelnen Fällen dennoch zu inhaltlichen Konflikten kommen kann, es also nicht immer für die konkreten Handlungsregeln gleichgültig ist, welche Begründung

17 | Um hier nicht Grund zur Annahme zu geben, ich deute die Kantische Ethik im Sinn der Diskursethik, sei darauf hingewiesen, dass in der Diskursethik die Grundlagen der Handlungsorientierung aus dem Prozess einer Vermittlung der Handlungsbeteiligten und Handlungsbetroffenen resultiert, während bei Kant die Vernunft des jeweils einzelnen hinreicht, unterschiedliche Interessen auszugleichen. 
jemand wählt. In den meisten Fällen jedoch wird sich eine hohe Übereinstimmung in den Prinzipien und Handlungsregeln ergeben; und im Fall einer Forderung nach der Ausrichtung von Handlungsorientierungen im Gesundheitswesen auf den Patienten ist das aber sicher keine Frage.

Zu den inhaltlichen Dichotomien kommt es erst, wenn die Positionen verkürzt werden, wie z.B. in den Sätzen: Der Pflichtethiker kümmert sich nicht um die Wirklichkeit. Oder: Der Utilitarist beurteilt moralisches Verhalten nur nach dem monetären Gewinn, weil das die einzigen Folgen sind, welche uns interessieren können. ${ }^{18}$

\subsection{Die Tugendethik}

Der tugendethische Ansatz, wie er von Aristoteles begründet wurde (vgl. für das Folgende: Fröhlich 2006, S. 18ff., sowie Fröhlich 2013), ist zuerst ebenso wie der Utilitarismus ein konsequentialistischer Ansatz, der allerdings mehrere Elemente in ihrem Stellenwert etwas verschiebt. Die Zielgröße ist wie im Utilitarismus das »Glück«, allerdings erstens individuell ausgerichtet (der Staat kommt erst in einem zweiten Schritt hinzu, die Individuen haben aber als Bürger die Bedingungen für den Staat, unter dem sie leben, zu gestalten), zweitens ist die eudaimonia bei Aristoteles kein Zustand, sondern

18 Im Übrigen ist das Geld für Mill ein relativer Wert für sich selbst. Was wie ein Widerspruch klingt, meint jeweils eine andere Ebene: Gegenüber dem Glück steht es relativ neben anderen Gütern oder Tugenden. Da wir aber die Erfahrung gemacht haben, dass Geld uns Sicherheit gibt, und die wiederum einen Teil unserer Zufriedenheit und damit auch unseres Glücks ausmacht, erstreben wir das Geld irgendwann auch um seiner selbst. Das gilt genauso für andere Vorzüge: das Glück ist demnach "kein abstrakter Begriff, sondern ein konkretes Ganzes, und dies [Tugend, Geld, Ehre, Ruhm, Liebe zur Musik, Wunsch nach Gesundheit usf.; GF] sind einige Teile dieses Ganzen [des Glücks; GF]“ (Mill 2006, S. 113; vgl. auch Fröhlich 2010, S. 248). 
ein Strebensziel, das - konsequent betrachtet - gar nicht erreichbar ist. Zwar kennt Aristoteles die Bedingungen für ein individuelles, soziales und politisches Wohlergehen, die eudaimonia ist aber wesentlich dynamisch durch das menschliche Handeln selbst geprägt. Die Bedingungen für das Handeln sind dann nicht nur in den äußeren Verhältnissen, sondern auch in der inneren Haltung fundiert. Ethisches Verhalten resultiert in der Tugendethik nicht so sehr aus der Handlung, sondern aus der inneren Haltung, auch wenn diese sich auf die Handlung überträgt. Tugenden sind Eigenschaften der menschlichen Seele, durch die wir, wenn wir diese angesichts der Verhältnisse, die Aristoteles mit seinem »wann man soll und wobei man es soll und wem gegenüber und wozu und wie« charakterisiert, ${ }^{19}$ bestmöglich einsetzen, auch das ethisch Geforderte erfüllen und damit die eudaimonia erreichen können. Das Ziel des tugendethischen Ansatzes, die eudaimonia, liegt aber in der Handlung selbst und nicht in ihrem Ergebnis, das schon gar nicht nur in einem Zustand des Wohlbefindens besteht. Eine solche Lesart schließt der aristotelische Text definitiv aus. ${ }^{20}$

19 | Die bekannte Stelle aus der Nikomachischen Ethik lautet: "Denn sie [die ethische Tugend; GF] befaßt sich mit den Leidenschaften und Handlungen, und an diesen befinden sich Übermaß, Mangel und Mitte. So kann man mehr oder weniger Angst empfinden oder Mut, Begierde, Zorn, Mitleid ohne überhaupt Freude und Schmerz, und beides auf eine unrichtige Art; dagegen es zu tun, wann man soll und wobei man es soll und wem gegenüber und wozu und wie, das ist die Mitte und das Beste, und dies kennzeichnet die Tugend. Ebenso gibt es auch bei den Handlungen Übermaß, Mangel und Mitte. Die Tugend wiederum betrifft die Leidenschaften und Handlungen, bei welchen das Übermaß ein Fehler ist und der Mangel tadelnswert, die Mitte aber das Richtige trifft und gelobt wird. Und diese beiden Dinge kennzeichnen die Tugend. So ist also die Tugend ein Mittelmaß, sofern sie auf die Mitte zielt" (NE II,5 1106b 16-28).

20 | Bevor Zweifel aufkommen, ob der Aristotelische Ansatz noch als konsequentialistisch aufgefasst werden kann, sei Folgendes klar gestellt: Aristoteles legt den Ausgangspunkt seiner ethischen Überlegungen in die 
Wie der Utilitarismus ist die aristotelische Tugendethik ebenso eine Traditionsmoral, die auf den überkommenden Verhaltensweisen aufbaut, die dabei zugrunde liegenden Prinzipien aber nicht begründen kann. Der Schwerpunkt der Moralbegründung liegt auf der Bestimmung des richtigen Strebensziels für die Lebensführung. Die Tugenden sind nur die Weisen, wie es dem Menschen möglich ist, das korrekte Ziel im Handeln auch anzustreben. Eine besondere Verstehensschwierigkeit des aristotelischen Ansatzes liegt in der Vorstellung, dass wir das Strebensziel des menschlichen Handelns nicht als Ergebnis aus den Folgen der Handlung betrachten dürfen, sondern schon im Handeln selbst realisieren (vgl. Fröhlich 2013).

\section{ZUR VEREINBARKEIT VON MORALISCHEN, ÖKONOMISCHEN, RECHTLICHEN UND MEDIZINISCHEN WERTEN}

Keine dieser drei Moralbegründungstypen gibt exakt an, was wir unter allen möglichen Bedingungen zu tun haben. Es ist klar nachvollziehbar, dass ein solches Unterfangen auch definitiv zum Scheitern verurteilt wäre. Alle Versuche achten aber darauf, die konkreten Handlungsbedingungen in den Überlegungen zum guten Handeln $\mathrm{zu}$ berücksichtigen, und alle wissen um die Vielfalt der Bedingungen und die unterschiedlichen Aspekte der Folgen unserer Hand-

Frage, was wir als Ziel des Handelns erstreben, insofern ist das eine Ausrichtung auf die Konsequenzen unserer Handlungen, also ist der Ansatz als konsequentialistisch einzustufen. Seine Analyse ergibt dann, dass das Ziel des menschlichen Handelns im Vollzug eines bestimmten und voraussetzungesreichen Handlungstyps besteht, den er als eudaimonia bezeichnet. Vollzug und Handlungsziel sind damit identisch. Daraus geht schon hervor, dass Aristoteles einen ganz anderen und anspruchsvolleren Handlungsbegriff vertritt, sich aber auch der Reduktion von Handlungen auf nur positiv feststellbare Ergebnisse ("nur was hinten rauskommt, zählt») nicht schuldig macht. 
lungen. Was vielleicht keiner der Typen recht zum Thema macht, ist, dass die Wünsche des Menschen durchaus in ganz verschiedene Richtungen gehen, sich ändern, überlagern, umstrukturieren usf. Auch der Interessens- und Bedürfnisbegriff im Utilitarismus, der einer Berücksichtigung von Wünschen noch am nächsten kommt, ist viel zu grob und zu formal. Eine Verbindung zwischen den Tatsachen in der Welt und den unterschiedlichen Bedürfnissen des Menschen versucht kaum einer der Ethiktypen wirklich zu entwickeln.

Dennoch ergeben sich, wenn wir diese Begründungen auf die Bereiche der Medizin, des Rechts und der Ökonomie anwenden, um so eine Verhältnisbestimmung der Ethik zu diesen Bereichen zu versuchen, unterschiedliche Schwerpunktsetzungen. Die Bereiche selbst beinhalten schon verschiedene Wertsetzungen, die sich in Wertsetzungsgruppen bei den Vertretern der Bereiche auffinden lassen. Aus dem instrumentellen Vernunftbegriff des Utilitarismus und seiner Ausrichtung auf die Handlungsfolgen, respektive des Nutzens, gründet sich die ethische Verantwortung in erster Linie auf die Gesellschaft, aber im ärztlichen Handeln eben auch auf den einzelnen Patienten, ${ }^{21}$ in dessen Gesundung das Ziel des Handelns besteht, während sich in der Pflichtethik, in der die Vernunft für die Prinzipienstruktur verantwortlich ist, bestimmte Regelstrukturen im Arzt-Patienten-Verhältnis etablieren müssen. Die Tugendethik mit ihrem universellen Vernunftbegriff setzt den Schwerpunkt dagegen in erster Line auf das ärztliche Ethos, das gegenüber dem Patienten Respekt, Empathie, Redlichkeit und Fürsorge entwickelt.

Auch in der Bewertung ökonomischer Zusammenhänge sind sich diese ethischen Theorien weitgehend einig: Geldäquivalente dienen in erster Linie der Erfüllung der dem System gestellten Aufgaben. Der am ehesten ökonomisch argumentierende Utilitarismus hält Wachstumsgrenzen an Kapitalakkumulationen ohnehin für

21 Hier ist klarerweise festzustellen: Es geht dabei in erster Linie um den Nutzen des Patienten, nicht um den des Arztes, seines Chefs, der Klinik, der Gesundheitssysteme oder der in Gesundheitsfragen involvierten Vertreter, ob diese aus der Politik, den Verbänden oder den Versicherungen kommen. 
notwendig, da solche niemals dem Allgemeinwohl dienen werden; vor allem aber wenn es darum geht, nicht-ökonomische Fortschritte - und das Gesundheitssystem wird durch seine anders gelagerte Aufgabenstellung im Wesentlichen als ein nicht-ökonomisches System bestimmt werden müssen - zu erzielen, also z.B. in der Patientenversorgung, können ökonomische Größen keine Rolle mehr spielen, schon gar nicht die entscheidende.

Ein ganz ähnlicher Befund ergibt sich für die Rechtsverhältnisse. Das Recht, so fordern diese Moralsysteme allesamt, dient der einheitlichen Regelung bestimmter Handlungsvollzüge, und bietet insofern Sicherheit, sich auf bestimmte Verfahren verlassen zu können. Diese Verfahren werden wieder durch die Aufgabenstellung des betrachteten Bereichs inhaltlich aufgeladen. Dass die Ökonomie die rechtlichen Regelungen dominiert, würde ein absolut sachfremdes Regelungsvorgehen zur Folge haben, weil die Ökonomie nur Teil des Systems, aber nicht deren zentrale Aufgabe darstellt. Sehr viel schwieriger gestaltet sich die Einordnung naheliegenderweise bei der Verhältnisbestimmung von Recht und Medizin, weil therapeutische Maßnahmen und die Möglichkeit, diese auch durchzuführen, den Kernbereich des Gesundheitssystems ausmachen. Daran, dass rechtliche Regelungen bestimmte Eingriffe als illegal qualifizieren, haben wir uns im Grunde aber schon gewöhnt. Es bedürfte einer eingehenderen Diskussion, wie solche Verbote begründet werden können, es besteht aber der Verdacht, dass diese wiederum moralische Gehalte aufweisen.

Insgesamt sind diese Orientierungen also nicht so weit von einander entfernt, wie häufig angenommen wird - und sie thematisieren in ihren ethischen Forderungen alle sehr stark eine Ausrichtung auf die Belange von Patienten. Die folgende Übersicht (Tabelle 4) auf der nächsten Seite kann das eben Ausgeführte noch verdeutlichen: ${ }^{22}$

22 | Erweiterte Fassung der tabellarischen Übersicht aus Fröhlich, Rogler 2005. 


\begin{tabular}{|c|c|c|c|c|c|}
\hline & 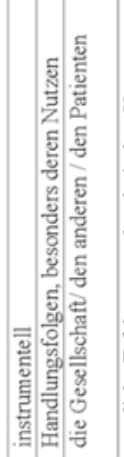 & 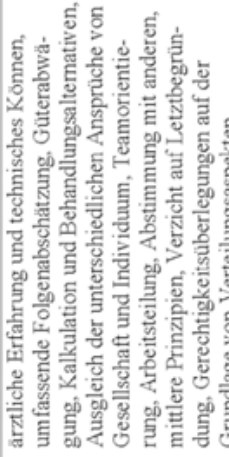 & 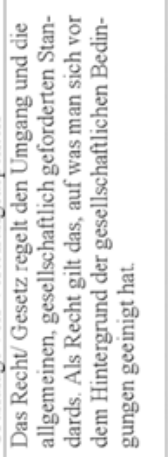 & 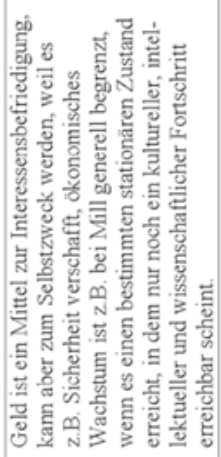 & \\
\hline & 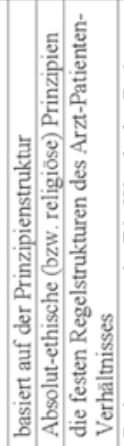 & 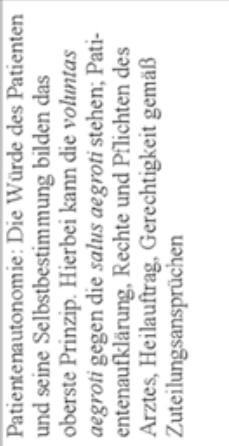 & 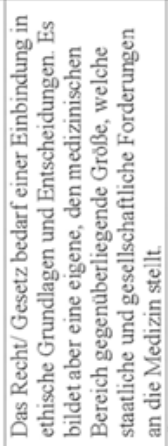 & 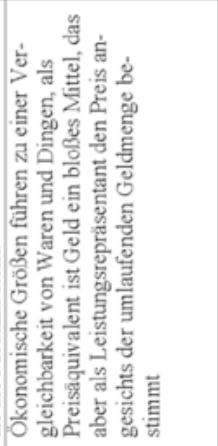 & \\
\hline & 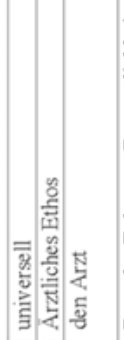 & 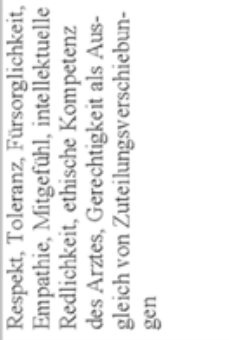 & 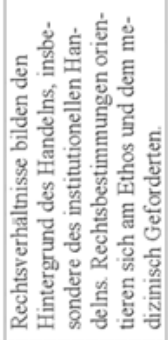 & 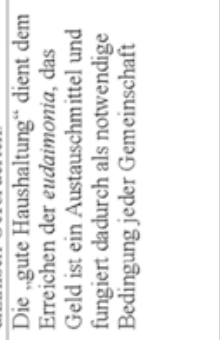 & \\
\hline & 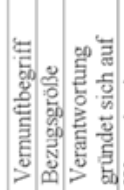 & & 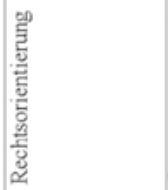 & 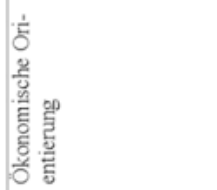 & \\
\hline
\end{tabular}

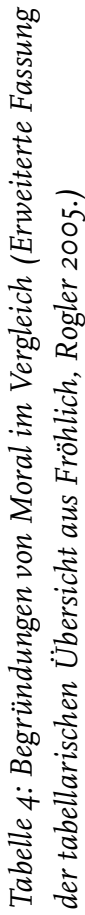


Für die Bereichsstrukturen der Medizin empfiehlt sich also die geradezu universal zu verstehende Ausrichtung auf das Arzt-PatientenVerhältnis. Vor allem nachdem die neuere Gesetzeslage verstärkt die Autonomie des Patienten betont, ist nicht einzusehen, warum die Ökonomie dies konterkarieren sollte. Der sinnvolle Einsatz der Mittel ist ein Gebot, das ebenso aus jedem ethischen Ansatz folgt, aber die Zielgröße, wenn es um die Zuteilung von Gesundheitsleistungen gehen soll, darf nicht von außen, also durch die Ökonomie, allein definiert werden, sondern von dem jeweiligen Aufgabenbereich, und der liegt in der Medizin in der Versorgung von Patienten - wie in allen ethischen Ansätzen im Grunde gefordert wird.

Diese Individualbetrachtung ist freilich nicht ohne Schwierigkeiten, wenn wir bedenken, dass unter der gesellschaftlichen Dimension die Mittel nicht an einzelne zugewiesen werden (obwohl das zuletzt durch das Handeln des Arztes doch geschehen sollte), sondern in globalen Budgets, nach Krankheiten sortiert, nach Durchschnitts- oder Mindestliegezeiten usf. festgelegt werden sollen. Das wird auch immer ein Problem bleiben. Aber die Ausrichtung der ganzen Organisation auf ökonomische Größen statt auf die Aufgabe des Systems, Leidenden zu helfen, wird dieses Problem in keiner Weise lösen können.

Eine derart radikale Konzentration der Ethik auf die individuelle Entscheidung hätte den klaren Vorteil, die Verantwortungsbezüge des Handelns besser einholen und zuordnen zu können. Es entsteht dabei aber die Frage, wie vor einem solchen Hintergrund das institutionelle Handeln, wie es bei politischen Entscheidungen gang und gäbe ist, überhaupt eingeholt werden kann. Umgekehrt wird es allerdings auch nicht von der Hand zu weisen sein, dass Institutionen keine anonymen Größen sind, sondern dass auch im institutionellen Kontext Entscheidungen von einzelnen Akteuren getroffen werden, auch wenn dabei die Abhängigkeiten der jeweiligen Stellung des Akteurs, die Relation zu anderen verantwortlichen Akteuren, die potentielle Teilung der Verantwortung und wohl noch einiges mehr bedacht werden muss. 
Institutionelle Entscheidungen sind zumeist mit unterschiedlicher Regelungsdichte bureaukratisiert. Der Sinn eines solchen Aufwands liegt in der inhaltlichen Absicherung und der Kontrolle von Handlungsabläufen. Die Frage ist, ob solche formal und inhaltlich mehrstufigen Entscheidungskomplexe vollständig automatisiert werden können (was häufig als Wunsch oder Ziel bureaukratischer Eingriffe erscheint) oder ob im Kontext menschlicher Handlungen auf Entscheidungsspielräume nicht verzichtet werden kann, ob faktisch nicht, weil wir immer mit unvorhersehbaren Situationen rechnen müssen, in denen wir erwarten, dass überlegende Personen mit den Problemen adäquat (das heißt sachlich und verantwortungsvoll) umgehen, oder weil es gar nicht gewünscht wird. In solchen Bezügen wird auch die Verantwortung für die jeweilige Entscheidung angemahnt, ohne dass näher thematisiert werden würde, ob diese dann auch eine ethische Komponente beinhaltet.

Im Grunde entspricht das individualethische Programm auch dem Terminus der Ethik bei Aristoteles, der das öffentliche Handeln unter dem Begriff der Politik fasst, auch wenn er darunter in erster Linie das versteht, was wir heute als Verfassung oder Grundordnung bezeichnen würden, ohne das er etwas vom Recht wüsste, das erst die Römer erfunden haben. ${ }^{23}$ Im ethischen Kontext unterscheidet Aristoteles die Gerechtigkeit von der Tugend. Zwar gilt: »Beide sind dasselbe, aber ihr Begriff ist nicht derselbe, sondern sofern sie sich anderen gegenüber betätigt, ist sie die Gerechtigkeit; sofern sie ein bestimmtes Verhalten schlechthin ist, ist sie die Tugend « (NE V,3 1130a 12-14). Daraus geht hervor, dass schon Aristoteles Schwierigkeiten hatte, den individual-ethischen Begriff vom öffentlich-politischen klar zu scheiden. Das »Betätigen anderen gegenüber« aber ist hier der Kern - und das kann nur die in erster

23 | Der griechische Begriff nomos umfasst das göttliche Recht, das Naturrecht und freilich auch das öffentliche Recht; letzteres ist aber nicht in unserem Sinne zu verstehen, sondern ist in letzter Hinsicht ein Blutrecht, in dessen Rahmen die Ahndung von Rechtverletzungen Sache der Familie oder der Sippe ist. 
Linie von den Maßnahmen und Regelungen Betroffenen meinen, also die Patienten.

\section{LITERATUR}

Aristoteles (2002): Die Nikomachische Ethik, übersetzt und mit einer Einführung und Erläuterungen versehen v. Olof Gigon, 4. Auflage. München: dtv (im Text abgekürzt mit NE).

Fröhlich, G.; Rogler, G. (2005): Das Regensburger Modell zur Ausbildung in Klinischer Ethik. Protokoll der Blockwoche für das 4 . Klinische Semester zur Ausbildung in Ethik der Medizin nach der neuen ÄAppO vom 27. Juni 2002 an der Medizinischen Fakultät der Universität Regensburg. In: Medizinethische Materialien des Zentrums für Medizinische Ethik der Ruhr-Universität Bochum, hg. v. Burkhard May, Hans-Martin Sass, Herbert Viefues, Heft 157, 2. Auflage.

Fröhlich, G. (2006): Nachdenken über das Gute. Ethische Positionen bei Aristoteles, Cicero, Kant, Mill und Scheler. Göttingen: Vandenhoeck \& Ruprecht.

Fröhlich, G. (2009): Ethische Beratung oder Therapie? Die konsiliatorische Grundstellung der Praktischen Philosophie und die Frage nach der Angewandten Ethik. psycho-logik. Jahrbuch für Psychotherapie, Philosophie und Kultur, Bd. 4: Lebensethik, 158175 .

Fröhlich, G. (2010): John Stuart Mill: Utilitarismus. In: Huber, H. (Hg.): Klassische Werke zur Philosophischen Ethik. München, Freiburg: Alber, 230-265.

Fröhlich, G. (2011): Form und Wert. Die komplementären Begründungen der Ethik bei Immanuel Kant, Max Scheler und Edmund Husserl. Würzburg: Königshausen und Neumann.

Fröhlich, G. (2012a): Ethische Überlegungen zur ärztlichen Versorgung in der Fläche. In: Baden-Württemberg aktuell. Newsletter des Hartmannbundes Landesverband Baden-Württemberg 2 (Juni 2012), 7 . 
Fröhlich, G. (2012b): Analyse und Funktion - Die Frage nach der Leitwissenschaft und die Philosophische Anthropologie. In: Fröhlich G (Hg.): Anthropologische Wege. Ulmer Stadthausvorträge. Nordhausen: Traugott Bautz GmbH, 185-225.

Fröhlich, G. (2012c): Fühlen - Handeln - Denken. Das Problem der Motivation und seine Bedeutung für die Ethik und die Klinische Ethikberatung. In: Frewer, A.; Bruns, F.; Rascher, W. (Hg.): Jahrbuch Ethik in der Klinik: Emotion und Ethik in der Medizin 5. Würzburg: Königshausen und Neumann, 21-41

Fröhlich, G. (2013): Die aristotelische eudaimonia und der Doppelsinn vom guten Leben. Archiv für Begriffsgeschichte 54: 21-44.

Galewicz, W. (1990): Wert und Gut. Zum phänomenologischen Wert-Pluralismus. Archiv für Begriffsgeschichte 33: 270-277.

Harth, M. (2008): Werte und Wahrheit. Paderborn: Mentis.

Hemel, U. (2007): Wert und Werte. Ethik für Manager - ein Leitfaden für die Praxis, 2. überarb. u. erweiterte Auflage. München: Hanser.

Hügli, A.; Schlotter, S.; Schaber, P.; Rust, A.; Roughley, N. (2004): Wert. In: Ritter, J.; Gründer, K.; Gabriel, G. (Hg.): Historisches Wörterbuch der Philosophie, Bd. 12: T-Z. Darmstadt: Wissenschaftliche Buchgesellschaft, 556-583.

Kaminsky, C. (2005): Moral für die Politik. Eine konzeptionelle Grundlegung der Angewandten Ethik. Paderborn: Mentis.

Kant, I. (2009): Kritik der praktischen Vernunft. In: Weischedel, W. (Hg.): Kant, Immanuel, Werkausgabe, Bd. 7, 19. Auflage. Frankfurt a.M.: Suhrkamp, 103-302. (im Text abgekürzt mit KpV).

Kant, I. (2009): Grundlegung zur Metaphysik der Sitten. In: Weischedel, W. (Hg.): Kant, Immanuel, Werkausgabe, Bd. 7, 19. Auflage. Frankfurt a.M.: Suhrkamp, 7-102 (im Text abgekürzt mit GMS).

Kant, I. (2012): Kritik der reinen Vernunft. In: Weischedel, W. (Hg.): Kant, Immanuel, Werkausgabe in zwölf Bänden, Bd. 3/4, 19. Auflage, Frankfurt a.M.: Suhrkamp. (im Text abgekürzt mit KrV). 
Kuhn, H. (1975): Werte - eine Urgegebenheit. In: Gadamer, H.G. und Vogler, P. (Hg.): Philosophische Anthropologie, Zweiter Teil (Neue Anthropologie Band 7). Stuttgart: Georg Thieme, 343-373.

Kutschera, F. von (2010): Wert und Wirklichkeit. Paderborn: Mentis.

Lichtblau, K. (2004): Wert/Preis In: Ritter, J.; Gründer, K.; Gabriel, G. (Hg.): Historisches Wörterbuch der Philosophie, Bd. 12: T-Z. Darmstadt: Wissenschaftliche Buchgesellschaft, 586-591.

Lotze, H. (1896): Mikrokosmus. Ideen zur Naturgeschichte und Geschichte der Menschheit. Versuch einer Anthropologie, Erster Band: Der Leib, die Seele, das Leben. 5. Auflage. Leipzig: Hirzel.

Lotze, H. (1905): Mikrokosmus. Ideen zur Naturgeschichte und Geschichte der Menschheit. Versuch einer Anthropologie, Zweiter Band: 4. Der Mensch. 5. Der Geist. 6. Der Welt Lauf. 5. Auflage. Leipzig: Hirzel.

Lotze, H. (1912): Metaphysik. Drei Bücher der Ontologie, Kosmologie und Psychologie (System der Philosophie II), hg. v. Georg Misch. Leipzig: Felix Meiner.

Mill, J.S. (2006): Utilitarianism/Utilitarismus. engl./dt., übers.u. hg. v. Dieter Birnbacher. Stuttgart: Reclam.

Schönrich, G. (2008): Option für eine philosophische Werttheorie. Allgemeine Zeitschrift für Philosophie 33(2): 97-124.

Sidgwick, H. (1909): Die Methoden der Ethik. Leipzig: Klinkhardt.

Smith, A. (2001): Der Wohlstand der Nationen. Eine Untersuchung seiner Natur und seiner Ursachen, aus dem Engl. v. Horst Claus Recktenwald, 9. Auflage. München: C.H. Beck.

Tappolet, C. (2009): Emotionen und die Wahrnehmung von Werten. In: Döring, S.A. (Hg.): Philosophie der Gefühle. Frankfurt a.M.: Suhrkamp, 439-461.

Vossenkuhl, W. (2006): Die Möglichkeit des Guten. Ethik im 21. Jahrhundert. München: C.H. Beck.

Weber, M. (1968): Die »Objektivität« sozialwissenschaftlicher und sozialpolitischer Erkenntnis. In: Weber, M.: Methodologische Schriften. Studienausgabe, mit einer Einführung von Johannes Winckelmann. Frankfurt a.M.: Fischer, 1-64. 



\section{Ressourcenzuteilung im Gesundheitswesen}

Zur Logik der Leistungssteigerung und

Effizienzmaximierung und ihren ethischen Grenzen

Arne Manzeschke

\section{Ressourcenallokation eine ethische Frage?}

Die Debatte um eine optimale Ressourcenallokation im Gesundheitswesen ist auf den ersten Blick eine rein ökonomische. Es ist nämlich die Ökonomie, welche im Rahmen ihrer Theoriebildung (oft auch Ökonomik genannt) und im Rahmen des praktischen Handelns der Aufgabe nachkommen muss, die Verwendung von Personal, Zeit oder Geld so zu gestalten, dass bei einem gesetzten Input ein maximaler Output bzw. bei gesetztem Output ein minimaler Input das optimale Verhältnis von Kosten und Nutzen bestimmt. Geld spielt hierbei die Rolle eines tertium comparationis ${ }^{1}$,

1 So schreibt Aristoteles in seiner Nikomachischen Ethik: "Daher muß alles seinen Preis haben; denn so wird immer Austausch und somit Verkehrsgemeinschaft sein können. Das Geld macht also wie ein Maß alle Dinge kommensurabel und stellt dadurch eine Gleichheit unter innen her. Denn ohne Austausch wäre keine Gemeinschaft und ohne Gleichheit kein Austausch und ohne Kommensurabilität keine Gleichheit. In Wahrheit können freilich Dinge, die so sehr voneinander verschieden sind, nicht kommensurabel sein, für das Bedürfnis ist es aber ganz gut möglich. Es muß also ein Eines geben, welches das gemeinsame Maß vorstellt, und zwar kraft positi- 
das den Vergleich zwischen eingesetzten Mitteln und erzielten Effekten objektiv widerspiegelt. Jenseits der Frage, ob Geld in diesen gesellschaftlichen Transaktionen nicht auch noch anders als nur als Wertmesser fungiert - z.B. als Zirkulationsmittel und als Mittel der Akkumulation $^{2}$ (vgl. Marx 1972, S. 143ff.) -, kommt mit der Frage der Ressourcenallokation dann aber auch eine moralische Dimension in den Blick: Die Entscheidung für die Option A und gegen die Option B basiert nicht allein auf dem rechnerischen Kalkül, dass der Nutzen von A größer als der von B ist, oder der Mitteleinsatz bei A geringer und effizienter als bei B ist; die Entscheidung für A basiert auch auf einer diesem Kalkül zugrunde liegenden Überzeugung, dass es vorzugswürdig ist, die nützlichere oder effizientere Variante zu wählen. ${ }^{3}$ Mit dem komparativen Prädikat nützlicher

ver Übereinkunft vorstellt, weshalb es auch Nomisma heißt, gleichsam ein vom Gesetz, Nomos, aufgestelltes Wertmaß. Denn alles wird nach inm gemessen " Aristoteles NE 1133b 16, S. 114.

2 | So schreibt Marx: "Da dem Geld nicht anzusehn, was in es verwandelt ist, verwandelt sich alles, Ware oder nicht, in Geld. Alles wird verkäuflich und kaufbar. Die Zirkulation wird die große gesellschaftliche Retorte, worin alles hineinfliegt, um als Geldkristall wieder herauszukommen. Dieser Alchemie widerstehen nicht einmal Heiligenknochen und noch viel weniger minder grobe res sacrosanctae, extra commercium hominum [...]. Der Trieb der Schatzbildung ist von Natur maßlos. Qualitativ oder seiner Form nach ist das Geld schrankenlos, d.h. allgemeiner Repräsentant des stofflichen Reichtums, weil in jede Ware unmittelbar umsetzbar. Aber zugleich ist jede wirkliche Geldsumme quantitativ beschränkt, daher auch nur Kaufmittel von beschränkter Wirkung. Dieser Widerspruch zwischen der quantitativen Schranke und der qualitativen Schrankenlosigkeit des Geldes treibt den Schatzbildner stets zurück zur Sysiphusarbeit der Akkumulation. Es geht inm wie dem Welteroberer, der mit jedem neuen Land nur eine neue Grenze erobert" (Marx 1972, S. 145, 147).

3 | Dieser These entspricht die Tatsache, dass von ökonomischer Seite immer wieder das Argument geäußert wird, dass es doch runethisch، sei, Ressourcen zu verschwenden. Auch wenn dieser Argumentation bisweilen 
bzw. effizienter sind keine rein ökonomischen Kategorien aufgerufen, sondern Vorstellungen von einem guten Leben, das mehr als nur den einen Menschen betrifft ${ }^{4}$, der diese Entscheidung trifft wenn es nicht überhaupt komplexere Strukturen sind, in den Entscheidungen von mehr als einem Menschen getroffen werden. ${ }^{5}$

Die lange Zeit etwas unkritisch vorgetragene Annahme, dass ökonomische Rationalität dafür sorge, dass die immer knappen Ressour-

eine immunisierende Funktion zukommen mag, mit der Ökonomen sich die Ein- und Ansprüche von Ethikern vom Leib halten, so ist inr zumeist nur intuitiv geäußerter Kern doch zutreffend. "Unwirtschaftliches Verhalten bedeutet in der Konsequenz die Verschwendung von knappen Ressourcen. Verschwendung in der Gesundheitswirtschaft bedeutet jedoch letztendlich unethisches Handeln“, Kölking 2007, S. 20.

4 | Vgl. hierzu Sedlácek 2012. Zur Frage nach Nutzen und Nützlichkeit im homo-oeconomicus-Modell vgl. Manzeschke 2010b. Dem individuellen Nutzen unterliegt eine moralische Wertsetzung, die aktuell schwer erkennbar scheint, weil in der ökonomischen Theorie allein noch die causa finalis, also der Nutzen, und inre Wirkursache, causa efficiens, übrig geblieben sind, während Zweck, causa formalis, und Materie, causa materialis, keine Berücksichtigung mehr finden; vgl. Brodbeck 1998, S. $156 \mathrm{ff}$.

5 | Damit ist ein wesentlicher Grund genannt, warum aktuell die Organisationsethik, die starke Bezüge zur Wirtschaftsethik aufweist, aber von ihr noch mal zu unterscheiden ist, solchen Aufschwung erfährt: Im Gegenzug zu einer primär vom handeInden Individuum her konzipierten Ethik besteht jene darauf, dass die institutionellen Bedingungen stärker als Konstituenten moralischer Entscheidungen berücksichtigt werden (vgl. hierzu grundlegend Krobath und Heller 2010; Krainer und Heintel 2010; Jäger und Coffin 2011; Hug 2009). Es kann an dieser Stelle nicht der Frage weiter nachgegangen werden, ob die Organisationsethik eine Fortentwicklung der Sozialethik der 1960er bis 1980er Jahre darstellt, oder ob sie ein eigenes und anderes Anliegen treibt, das sich besonders dem Umstand verdankt, dass Organisationen des Sozialsektors, die lange vom Ethos des Einzelnen bzw. dem Ethos einer bestimmten Berufsgruppe her ihre Entscheidungen rekonstruierten, hier an theoretische wie praktische Grenzen gestoßen sind. 
cen einer effektiven und effizienten Verwendung zugeführt werden und so ein Mechanismus gefunden sei, der die Gesellschaft an entscheidender Stelle von Reflexion und Entscheidung entlasten könnte, scheint sich zumindest in Teilen als unzutreffend herauszustellen. Die zyklisch wiederkehrenden Wirtschaftskrisen und zuletzt die Finanzkrise von 2008 haben die Rückfrage provoziert, ob »sich auf den Schauplätzen der internationalen Finanzwirtschaft ein effizientes Zusammenspiel vernünftiger Akteure oder ein Spektakel reiner Unvernunft vollzieht. Es ist jedenfalls nicht ausgemacht, ob der darin beschworene kapitalistische >Geist< verlässlich und rational oder schlicht verrückt operiert« (Vogl 2010, S. 7). ${ }^{6}$

Der ökonomische Mechanismus, Ressourcen über eine Preisbildung durch Angebot und Nachfrage zwischen den informierten Akteuren zu verteilen ${ }^{7}$, scheint zumindest für einige Sphären gesellschaftlichen Zusammenlebens eher dysfunktional zu sein.

Dem prinzipiellen Argument, warum die Frage der Ressourcenallokation nicht der Ökonomie allein überlassen werden kann, sollen im Folgenden Beobachtungen zur Seite gestellt werden, mit denen für eine weitergehende Berücksichtigung ethischer Aspekte plädiert wird. Gerade für den Bereich der gesundheitlichen Versor-

6 | Man mag einwenden, die Probleme des Finanzmarktes seien nicht identisch mit denen des Güter- oder Arbeitsmarktes, deshalb dürfe von der Dysfunktionalität des einen nicht auf die der anderen geschlossen werden. Das ist prinzipiell richtig, ebensowenig darf jedoch verkannt werden, dass vom Finanzmarkt ein starker Sog auf alle anderen Märkte ausgeht, denen sich diese nicht entziehen können. Bemerkbar macht sich dieser Sog zum Beispiel an der enormen Beschleunigung, die daraus resultiert, dass Transaktionen am Finanzmarkt sehr viel schneller durchgeführt werden können als anderswo, und an den Renditeerwartungen, die auch auf andere Märkte übertragen werden bzw. die Richtung der Kapitalströme diktieren. Vgl. zum Problem der Regulierung der Finanzmärkte Roubini und Minm 2010.

7 | Das Problem besteht auch dann noch, wenn man, wie in der Neuen Institutionenökonomik von asymmetrischen Beziehungen und unvollständigen Informationen ausgeht; vgl. Manzeschke und Brink 2010. 
gung in einer liberalen, demokratischen Gesellschaft, die sich an der prinzipiellen Gleichheit ihrer Glieder orientiert, ist es offenbar nicht zuträglich, Ökonomie und Ethik zu weit auseinander treten zu lassen.

\section{Marktliche Dynamik und Kostendämpfung}

Die politische Steuerung des Gesundheitswesens steht seit vielen Jahrzehnten vor dem Problem, dass eine allgemeine, gleiche und zeitnahe Versorgung der Bürgerinnen und Bürger mit Leistungen, die auf der Höhe des medizinisch-technischen Fortschritts sind, der Quadratur des Kreises gleichkommt. Einerseits sollen alle am medizinischen Fortschritt teilhaben können und bei Bedarf in den Genuss der entsprechenden Leistungen kommen. Auf der anderen Seite generieren solche Ankündigungen permanent steigende Erwartungen, Ansprüche - und Kosten. Wenn dann noch - durch den demographischen Wandel bedingt - die Zahl der Sorgebedürftigen steigt und deren Multimorbidität die Komplexität, Dauer und Kosten der Versorgung steigert, gleichzeitig aber die jüngeren Generationen als Beitragszahler und Fachkräfte für die Sorge ${ }^{8}$ zahlen-

8 | Ich wechsle hier bewusst zwischen den Begriffen "Versorgung" und "Sorge". Während der erste den sprachlichen Gewohnheiten der Branche folgt und vor allem den logistischen, organisationalen und auch ökonomischen Aspekt betont, stellt der zweite Begriff stärker auf das zwischenmenschliche Geschehen und die leibliche Dimension ab. Beide Aspekte sind wichtig und dürfen nicht gegeneinander ausgespielt werden. Ein Problem der aktuellen Diskussion ist es jedoch, dass sie vor allem den produktions- und distributionslogischen Aspekt der Versorgung thematisiert und darüber vergessen macht, dass es um die gut organisierte Sorge für Menschen geht, die in Teilen oder in Gänze nicht (mehr) für sich selbst sorgen können. Diese grundsätzliche Dimension darf nicht aus dem Blick geraten, will man Menschen als Menschen gerecht werden und sie nicht zu Objekten eines Produktionsprozesses degradieren. 
mäßig schrumpfen, dann ist auf den ersten Blick einsichtig, dass diese Konstruktion nicht aufgehen wird. Eine Absenkung der Leistungen anzukündigen, ist politisch ebenso unpopulär wie eine Steigerung der Beitragssätze - was zudem als volkswirtschaftlich problematisch angesehen wird, weil mit steigenden Beitragssätzen auch die Arbeitskosten ansteigen und somit die Konkurrenzfähigkeit auf einem globalen Markt gefährdet werden könnte. ${ }^{9}$ Stattdessen erscheint gegenwärtig eine konsequente Ökonomisierung des Gesundheitswesens als Ansatz, um zwischen der Skylla einer Verteuerung der Leistungen und der Charybdis einer Reduktion der Leistungen hindurchzusteuern. Ökonomisierung bedeutet danach die Gestaltung von Organisationen und Versorgungsprozessen nach effizienzorientierten, rationalen Kalkülen. Dabei lässt sich unterscheiden zwischen einer sachgemäßen und ethisch verantwortbaren Ökonomisierung, die neben dem ökonomischen Kalkül auch die Sachlogik des Handlungsfeldes als das Sachgemäße respektiert und ihr deshalb sogar Vorrang gewährt - und einer Form der Ökonomisierung, welche die Sachlogik zugunsten des ökonomischen Interesses nach maximaler Effizienz oder maximalem Profit in den Hintergrund treten lässt. Es mutet paradox an, dass ausgerechnet die >Ökonomisierung des Gesundheitssystemsく, die zu größerer Transparenz, Kostenkontrolle und Wirtschaftlichkeit beitragen soll, offenbar eine Ausweitung der Kosten und damit eine Verschärfung der Situation herbeiführt.

Ökonomisierung ist hier in zweifacher Hinsicht zu denken. ${ }^{10}$ Erstens bezeichnet sie einen Prozess, bei dem das Handeln im Be-

9 | Auch wenn die Steigerung der Versicherungsbeiträge für die Arbeitgeber gedeckelt wurde und diese Debatte auf den ersten Blick an Schärfe verIoren hat, bleibt das Problem in unserem Versicherungssystem bestehen, weswegen eine Maxime der politischen Steuerung bleibt, den Krankenversicherungssatz nicht oder nur minimal steigen zu lassen

10 | Der Begriff ,Ökonomisierung، schillert: Er wird einerseits von Ökonomen in programmatischer und positiver Konnotation verwendet, um die Notwendigkeit zu signalisieren, in praktisch allen Lebensbereichen ökono- 
reich der Gesundheitsversorgung als eine ökonomische Transaktion konzeptualisiert wird, die es ökonomisch rational zu gestalten gilt. Bis in die 1990 er Jahre galt der Soziale Sektor zumindest in weiten Teilen als Feld für staatliche Subventionen, auf dem unternehmerisch nichts zu lukrieren ist (vgl. Buestrich und Wohlfahrt 2008). Dass nun auf einem >Gesundheitsmarkt $<$ Renditen zu erwarten sind, und Unternehmen sowie Investoren sich hier betätigen, ist nur auf der Grundlage eines Paradigmenwechsels möglich. ${ }^{11}$ Dem liegt die Vorstellung zugrunde, dass sich die diagnostischen, therapeutischen und palliativen Tätigkeiten, ihre Akteure wie auch alle dabei eingesetzten Heil- und Hilfsmittel als Elemente eines Leistungserstellungsprozess verstehen lassen. Für diesen gilt - wie für alle anderen Produktionsprozesse auch -, dass er ökonomisch rational, d.h. effizient gestaltet werden muss. Auch wenn es Fragen des Grenznutzens gibt, kann man sich aus ökonomischer Sicht prinzipiell eine permanente Steigerung der Effizienz vorstellen. Zwar ist das Gesundheitswesen stark reguliert und entspricht damit gerade nicht einem echten Markt (vgl. Dietz 2011, bes. S. 291ff.), und doch lässt sich über die letzten Jahre sehr deutlich erkennen, wie die Ökonomisierung dazu beigetragen hat, das Leistungsvolumen aus-

mische Rationalität walten zu lassen, also eine rationale Kalkulation des Verhältnisses von Aufwand und Nutzen bei Transaktionen durchzuführen. Der Begriff wird andererseits aber auch in kritisch-ablehnender Weise verwendet, um genau diese Verfremdung einzelner Lebensbereiche oder Tätigkeiten durch ein ökonomisches Kalkül abzuwehren. Dazwischen hat sich in den vergangenen Jahren eine sozialwissenschaftliche Verwendung des Begriffs etabliert (vgl. hierzu Manzeschke 2011; Kettner und Koslowski 2011; Schimank und Volkmann 2008).

11 | Vgl. hierzu exemplarisch Nefiodow 2000. Nefiodow prognostiziert einen sechsten Kondratieffzyklus, der vor allem durch die Bedürfnisse und Basisinnovationen im Bereich der Gesundheitsversorgung, der Wellness und der ganzheitlichen Lebensführung verursacht werden wird. Aus gesundheitsökonomischer Perspektive vgl. Oberender et al. 2010. 
zuweiten. ${ }^{12}$ Neben der Kostenausweitung treten aber noch weitere Probleme auf: Niedergelassene Ärzte, die sog. Individuelle Gesundheitsleistungen (IGeL) ihren Patienten anbieten, tragen zur prinzipiellen Verunsicherung bei, welche Leistungen aus medizinischer Notwendigkeit und welche aus ökonomischer Opportunität >angeboten< werden. In Krankenhäusern lassen sich ähnliche Entwicklungen beobachten. ${ }^{13}$ Die medizinische Evidenz bei Therapieentscheidungen weicht zusehends der kundenorientierten Präferenz, wobei diese nicht unwesentlich durch den Rat der Professionellen mitbestimmt wird.

Auch im stationären Sektor lassen sich die Veränderungen beobachten, die zusehends als problematisch bewertet werden. Im September 2008 wurde von einer prominenten deutschen Tageszeitung mit Berufung auf staatsanwaltschaftliche Ermittlungen der Verdacht formuliert, das Gesundheitswesen sei »systematisch korrupt« (Bohsem 2009). Anlass waren 3.800 erfasste Betrugsfälle, in denen niedergelassene Ärzte ihre Patienten an Krankenhäuser bei der Einweisung >verkauft< hatten. Die Zahl benenne lediglich die Spitze des Eisberges, so die Ermittler. Es sei ein Geschäft auf

12 | Eine Gesundheitsbranche, die - politisch gewollt - als Jobmotor und Quelle der Wertschöpfung installiert wird, kann an ihrer eigenen Begrenzung kein Interesse haben; d.h. sie wird sich beständig ausweiten und weitere Umsätze, aber auch Kosten generieren, was dem politischen Ziel, diese Kosten im Gesundheitswesen einzudämmen, stracks zuwiderläuft. Berechnungen des Statistischen Bundesamtes zufolge waren es im Jahr 2010287 Milliarden Euro (www.bmg.bund.de/gesundheitssystem/gesundheitswirt schaft/bedeutung-der-gesundheitswirtschaft.html; Zugriff: 10. 12. 2012), darin eingerechnet sind noch nicht die sogenannten OTC-Produkte und gesundheitsnahen Ausgaben, die von den Bürgerinnen und Bürgern jenseits der Kranken- und Pflegeversicherung zusätzlich aufgebracht werden; Schätzungen nennen hier 400 Milliarden Euro Umsatz (www.abendblatt. de/ratgeber/gesundheit/article2329392/400-Milliarden-Euro-UmsatzDie-Gesundheitsbranche-boomt.html; Zugriff: 10. 12. 2012).

13 | Vgl. Heubel 2011; Braun et al. 2010; Dietz et al. 2007. 
Gegenseitigkeit zu beobachten, bei dem einweisende Ärzte von den Krankenhäusern bezahlt würden, weil diese >Kunden müssten. Umgekehrt entlassen die Krankenhäuser ihre Patienten an bestimmte Ärzte zur Nachsorge, was sich für diese finanziell auszahle, in Summe aber die Krankenkassen belaste, so der damalige Präsident der Bundesärztekammer Jörg-Dietrich Hoppe.

Betrachtet man die jüngste Berichterstattung (vgl. exemplarisch Faller und Grefe 2012) $)^{14}$, so wird man die inkriminierten Fälle nicht als statistische Ausreißer oder als notwendiges Durchgangsstadium betrachten dürfen, sondern man wird konzedieren müssen, dass ein strukturelles Problem besteht, und die ökonomischen Anreizsysteme - bei einzelnen sinnvollen Verbesserungen - insgesamt zu einer Ausweitung der Kosten einerseits und andererseits zu einer Erosion des professionellen Ethos und damit auch zu Einbußen in der Qualität beigetragen haben. Dieser Befund wird allenfalls diejenigen überraschen, die eine schlichte Übertragbarkeit industrieller Fertigungslogik auf den Gesundheitssektor für möglich, sinnvoll und sachgerecht erachtet haben.

So richtig es ist, die ökonomische Dimension bei allen Tätigkeiten im Blick zu behalten, so problematisch wird es doch, wenn diese Dimension dominiert und vergessen macht, dass sich im Fall gesundheitlicher Sorge nicht Leistungsanbieter und Leistungsempfänger als Agenten eines Warentausches begegnen. So richtig es ist, Strukturen und Prozesse im Gesundheitswesen daraufhin zu durchleuchten, ob und in welchem Maße hier Ineffizienzen, Fehlallokationen oder gar Korruption ${ }^{15}$ herrschen, und diese dann $\mathrm{zu}$ beseitigen, so problematisch ist es andererseits, wenn dabei die spe-

14 | Ich nenne hier nur diesen einen Zeitungsbericht, weil er meines Erachtens sehr gut ein Dilemma in die breite Öffentlichkeit transportiert, das von der Forschung schon sehr viel länger thematisiert wird - allerdings ohne dass dies zu sachlichen Änderungen geführt hätte (vgl. Braun et al. 2010; Heubel et al. 2010; Huster 2011).

15 | Transparency International hat in seinem Jahresbericht 2006 zur Korruption im deutschen Gesundheitswesen festgestellt, dass etwa $10 \%$ des 
zifische Fachlichkeit der Gesundheitsberufe umdirigiert wird, eher ökonomischen Interessen zu folgen als der sachlich-fachlichen Notwendigkeit.

Zweitens ist Ökonomisierung zugleich als ein Prozess zu verstehen, bei dem, angetrieben durch eine kapitalistische Zinsdynamik, eine beständige Ausweitung dieses Prozesses entsteht, der Markt also sich nur erhalten kann, wenn er sich beständig vergrößert (vgl. Deutschmann 2009; Polanyi 1978). Diese Dynamik ist kein Naturgesetz, dem sich menschliche Gesellschaften sachzwanghaft ausgesetzt sehen müssen, sondern ein von diesen Gesellschaften selbst mehr oder minder bewusst gesetzter Handlungsrahmen, der sich dann sehr wohl verselbständigen und quasi-objektiv den Akteuren gegenübertreten kann. ${ }^{16}$ Entscheidend ist, dass unterschieden wird zwischen echten Sachgesetzlichkeiten, die deshalb bestehen, weil man haushalten muss, und solchen Sachzwängen, die durch eine bestimmte Wirtschaftsweise erzeugt werden, die aber auch geändert werden können.

\section{Ethos und Ökonomie bei den Professionellen im GesundheitsWesen}

Professionelle, die im Gesundheitswesen arbeiten und kranke, behinderte, pflegebedürftige Menschen umsorgen, tun dies im Idealfall nicht wegen exorbitanter Verdienstchancen, sondern weil sie >mit Menschen zu tun haben bzw. ihnen helfen wollen<. Der - insbesondere in der Pflege - nicht üppige Verdienst ${ }^{17}$ wird jedoch von

jährlichen Budgets im Gesundheitswesen (2005: 240 Milliarden Euro) in Korruptionskanäle fließen (Transparency International 2006).

16 | Vgl. exemplarisch Ulrich 2008, 2010; und die Ausführungen zu Arthur Rich weiter unten.

17 | Eine Ausnahme hinsichtlich Verdienst und fachlicher Anerkennung stellt die Intensivpflege dar (vgl. Deutsches Institut für Pflegeforschung 2012). Zur Anerkennung der Pflegearbeit vgl. Kumbruck et al. 2010. Zur 
einer anderen >Kapitalsorte flankiert, die offenbar immer noch einen wichtigen Anreiz liefert, in diesem Sektor zu arbeiten: Sich dem Menschen zuzuwenden und ihm und damit der ganzen Gesellschaft in gewisser Weise $z \mathfrak{u}$ >dienen< (vgl. hierzu den erhellenden Essay von Schuh 2007), d.h. eine sinnvolle Arbeit zu verrichten, findet seine Motivation für viele Beteiligte in besonderer Weise darin, dass kleine Gesten der Dankbarkeit, Fortschritte in der Genesung oder auch das gemeinsame Aushalten existentieller Momente (Geburt, Sterben, schwere Diagnosen) als sinnvoll erlebt werden. Es ist wohl nicht zuletzt diese Dimension sinnhaften Handelns, die von vielen Professionellen angegeben wird, wenn sie trotz einer belastenden Tätigkeit, ungünstiger Arbeitszeiten und zum Teil vergleichsweise geringer Löhne in diesem Beruf arbeiten (vgl. hierzu DGB Index).

Sinnhafte, selbstwirksame und dem Patienten zugewandte Tätigkeit, so lässt sich zusammenfassend formulieren, sind wesentlich motivierende Faktoren, wenn Menschen sich in diesem Bereich beruflich engagieren. Diese Motivation erfährt jedoch starke Einschränkungen in dem Maße, wie patientenferne Tätigkeiten (z.B. Dokumentation) und allgemeiner Zeitmangel den Kontakt zum Patienten einschränken. Diese Situation führt nicht nur zu Qualitätseinbußen in der Versorgung, weil für eine ausreichende Beobachtung des Patienten nicht genügend Zeit bleibt (vgl. Isfort und Weidner 2010), sie resultiert auch in einer sinkenden Motivation der Professionellen, weil ihre Tätigkeit zunehmend von ökonomischen Erwägungen strukturiert wird, und diese oft genug gegen die fachlichen Erwägungen zu stehen kommen. Was Freidson einmal das >Versprechen< einer Profession genannt hat (Freidson 1979), nämlich der Gesellschaft gegenüber als Garant fachlicher Expertise für die durch Krankheit oder Unfall in Not und Abhängigkeit Geratenen zu agieren, wird zunehmend fraglich. In dem Maße, in dem die Professionellen des Gesundheitswesens in einen Interessenkonflikt

Arbeitsbelastung unter Klinikärzten vgl. Kiesel et al. 2010; zur Arbeitszufriedenheit unter niedergelassenen Ärzten vgl. Johnson und Sattler 2005. 
geraten zwischen dem Wohl der Patienten und den ökonomischen Interessen ihrer Institutionen, in dem Maße wird das professionelle Versprechen unglaubwürdig - ein Problem, mit dem sich dann nicht nur die einzelnen Patienten oder Professionellen konfrontiert sehen. Zunehmend wird dieses Problem auch in der Gesellschaft als Problem der Gesellschaft erkannt. Die Forderung nach einer gerechten Ressourcenverteilung ist meines Erachtens auch ein Indiz dafür, dass die Verunsicherung in der Gesellschaft gewachsen ist, ob es in diesem System überhaupt gerecht zugeht.

Der Ökonomisierung der ärztlichen und pflegerischen Tätigkeiten liegt zunächst eine völlig plausible Überlegung zugrunde: Prozesse und Strukturen sollen so organisiert werden, dass die Professionellen ihre genuinen Fachkompetenzen möglichst effektiv einsetzen können. Diesem Anliegen wird man - auch ohne weitere Kenntnis des Gesundheitsbetriebs - prinzipiell zustimmen können. Ein näherer Blick auf das System zeigt, dass Ineffizienzen durch unangemessenen Personaleinsatz (z.B. hochqualifizierte Pflegekräfte übernehmen Transportdienste im Krankenhaus) oder unzureichende Prozessorganisation (z.B. bei der Auslastung von Operationssälen) immer wieder anzutreffen sind und eine Ökonomisierung im Sinne eines sachgemäßen effektiven und effizienten Mitteleinsatzes berechtigt erscheinen lassen. Problematisch wird in meinen Augen diese Effektivitäts- und Effizienzsteigerung dort, wo insbesondere letztere dazu führt, dass ökonomisch interessante, therapeutisch aber fragwürdige Maßnahmen ergriffen werden bzw. notwendige Maßnahmen aus ökonomischen Erwägungen unterlassen werden. In beiden Fällen soll das Personal - auch gegen die eigene fachliche Kenntnis - ökonomisch orientiert arbeiten.

Die Zunahme patientenferner Tätigkeiten, die es den Professionellen erschwert, für ihre Patienten da zu sein und aufgrund der regelmäßigen Beobachtung therapeutisch angemessen $\mathrm{zu}$ agieren, ist das eine Moment, das die Motivation der Professionellen absenkt. Das andere Moment ist die kognitive und auch emotionale Dissonanz, in der sich Professionelle erleben, wenn sie am Patienten gegen ihre eigene fachliche Expertise arbeiten müssen. 
Es kommt nun noch ein drittes Moment hinzu, dass ich als >pervertierte Supererogation < bezeichne (Manzeschke 2010a). Damit ist das Phänomen gemeint, dass Professionelle im Gesundheitsbereich sich >für< ihre Klientel (Patientin, Bewohner, Pflegebedürftige u.a.) auch über das gebotene Maß hinaus einsetzen. Damit sind nicht nur die vielen Überstunden gemeint ${ }^{18}$, sondern viel mehr noch die Bereitschaft, jenseits der vertraglich vereinbarten und moralisch forderbaren Leistungen mehr für diese Klientel zu tun. Die Möglichkeit, sich selbstbestimmt und freiwillig über das gebotene Maß hinaus für die Patienten zu engagieren, wird für die Professionellen durch die permanente Steigerung der Effizienz jedoch immer mehr eingeengt - man könnte hier in Analogie von einem ,crowding-out-Effekt< sprechen: Das organisationale Bemühen um maximale Effizienz der Strukturen und Prozesse unterläuft die Möglichkeiten des individuellen Akteurs, sich über das forderbare Maß zu engagieren. Die positive Intention des Ökonomisierungsprozesses zeitigt ab einem nicht eindeutig zu bestimmenden Punkt einen kontraproduktiven Effekt. Der Versuch, das gesamte Potenzial der Mitarbeitenden im Sinne eines effizienten Ressourceneinsatzes abzurufen, führt zu dem gegensätzlichen Effekt, dass immer weniger Freiraum bleibt für ein Engagement über das vertraglich vereinbarte Maß hinaus. Damit werden aber gerade nicht alle >Effizienzreserven mobilisiert, sondern es werden im Gegenteil vorhandene, aber organisational nicht stratifizierbare Potenziale ausgesteuert. So wenig sich ein überobligates Engagement moralisch einfordern lässt, so wenig lässt es sich durch Controlling- oder Anreizsysteme ökonomisch herbeisteuern. Je mehr dies versucht wird, desto stär-

18 | Die physische und psychische Arbeitsbelastung der Pflegekräfte im Krankenhaus ist seit Einführung der DRG deutlich gestiegen. Dies ist nicht allein auf einen starken Abbau von Pflegekräften aufgrund von Überkapazitäten zurückzuführen, sondern auch durch Arbeitsverdichtung und -vermehrung. 2006 zählte das Deutsche Institut für Pflegeforschung 9 Millionen Überstunden, die etwa 5.000 Vollzeitäquivalenten entsprechen (Isfort und Weidner 2007, S. 5). 
ker schwindet der Freiraum, in dem solches Engagement überhaupt möglich wäre. Schwindet der Freiraum, so sinkt die Motivation und die Möglichkeit der Mitarbeitenden, sich selbstbestimmt und selbstwirksam in die Arbeit einzubringen. Darüber hinaus erweist sich eine solche permanente Effizienzsteigerung als dysfunktional, weil sie die Mitarbeitenden von ihrer Klientel räumlich entfernt, ihnen nicht-patientenbezogene Tätigkeiten auferlegt und im schlechtesten Fall die Belastbarkeit der Mitarbeitenden nicht berücksichtigt (Manzeschke und Brink 2010).

Es gibt also hinreichend viele Hinweise dafür, dass das Gesundheitswesen, so wie es derzeit strukturiert ist, weder ökonomisch noch ethisch wirklich befriedigende Ergebnisse hervorbringt. Es zeigt sich außerdem, dass das Ethos der Professionellen ebenso von ökonomischen Anreizstrukturen ausgesteuert wird wie auch deren Fachlichkeit, und die moralischen Erwartungen der Gesellschaft auf eine gerechte und sachgemäße Gesundheitsversorgung werden enttäuscht. In einem letzten Schritt soll nun überlegt werden, inwiefern ethische Überlegungen einer dem Handlungsfeld angemessenen Ökonomie dienen können.

\section{Ethische Kriterien: SAchgemäsS UNd Menschengerecht (ARthur Rich)}

In der deutschsprachigen Wirtschaftsethik hat der Schweizer Theologe Arthur Rich (1910-1992) bereits früh nach allgemeinen Kategorien gesucht, mit denen sich eine Einbettung ökonomischer Transaktionen in gesellschaftliche Zusammenhänge sowohl theoretisch begründen wie auch praktisch durchführen lässt (vgl. bereits Wünsch 1927, S. 388ff.). ${ }^{19}$ Rich erkannte im Sachgemäßen und

19 | Wünsch betont aus einer wertethischen Perspektive die "dienende" Funktion der Wirtschaft, sie habe eine "teleologische Autonomie" und sei so anderen, höheren Werte ein- und zugeordnet. Eine Verabsolutierung des wirtschaftlichen Wertes führe zu einer Umkehrung der Werte. Die „Dämonie 
im Menschengerechten die einander irreduzibel ergänzenden Momente zur Evaluation und Normierung ökonomischer Handlungen (Rich 1991, S. 76ff.).

Das Sachgemäße hat nach Rich den Charakter des Objektiven. So ist z.B. die Funktionstüchtigkeit einer Brücke objektiv abhängig von der Berücksichtigung physikalischer, genauer statischer Gesetze. Um eine Brücke gut bauen bzw. die Güte einer Brücke beurteilen zu können, müsse man keine ethischen Kategorien heranziehen, sondern fragen, ob die richtige, d.h. sachgemäße Anwendung der hier geltenden Gesetze stattgefunden habe. Das Sachgemäße ist so gesehen ethisch indifferent. Gleichwohl sei es nötig, das sachgemäße Handeln auch ethisch zu verhandeln; denn ein nicht sachgemäßes Handeln könne negative Folgen für Menschen und Umwelt haben.

"Man kann also sagen, daß hier das wirklich Sachgemäße trotz seinem prinzipiell nichtethischen Charakter, dem zu entsprechen vermag, was auch die Ethik als Verpflichtung zum Menschengerechten will« (Rich 1991, S. 76).

So sehr eine ethische Betrachtung von Sachverhalten die jeweiligen sachgemäßen Gesichtspunkte berücksichtigen müsse, dürfe sie sich davon jedoch nicht primär leiten lassen. Das Sachgemäße müsse stets an das Menschengerechte gebunden bleiben, und dieses könne nicht aus jenem abgeleitet werden. Es gehe also nicht darum, »ob ein so oder anders strukturiertes gesellschaftliches Konzept funktionsfähig oder effizient sei, sondern vor allem, wie es bei

des Wirtschaftswertes“ (Wünsch 1927, S. 389) bedürfe einer begriffsgeschichtlichen Aufklärung über die Grenzen des Wirtschaftlichen und seiner Autonomie. Das werde, so Wünsch, in der neueren Nationalökonomie, die er mit den Namen Max Weber, Karl Diehl, Othmar Spann, Gustav Cassel und Theodor Brauer verbindet, auch geleistet. Hier habe man erkannt, "wie sehr die Nationalökonomie, um praktisch fruchtbar zu werden, der Ethik oder Weltanschauung, bedarf bei allerdings scharfer methodischer Trennung der beiderseitigen Sachgebiete" (Wünsch 1927, S. 390). 
der Verwirklichung mit der Qualität des Menschlichen stehe, ob sie dadurch gemehrt oder gemindert werde« (Rich 1991, S. 73). Man könne also, um es mit Max Weber zu formulieren, auf der Seite des Sachgemäßen sehr wohl wertfrei argumentieren, jedoch komme man bei der Gesamtbetrachtung eines Phänomens aus ethischer Perspektive nicht um ein wertendes Moment umhin, das mit dem Kriterium des Menschengerechten aufgegeben sei.

Bezogen auf die Mittelverteilung im Gesundheitswesen lässt sich sagen, dass Effizienz allein kein zureichendes Kriterium darstellt. Es muss ebenso um die Sachgemäßheit im Sinne der fachlichen Expertise gehen und drittens um das Menschengerechte.

Lässt sich das Sachgemäße als deskriptiv verstehen, sofern mit ihm die sachlichen Momente eines Phänomens allgemeingültig beschrieben werden, so hat das Menschengerechte demgegenüber den Charakter des Normativen oder Präskriptiven. Es beschreibt, was sein soll; z.B. das friedliche und gerechte Zusammenleben der Menschen, oder - bezogen auf das Gesundheitswesen - eine Versorgung, die auf die Not des Menschen sachgemäß eingeht und diese Not nicht ausnützt. Kriterien des Menschengerechten sind Normen, die präskriptiv sagen, »was im prinzipiellen Sinne ethisch zu gelten hat«. Maximen des Menschengerechten sind Normen, die sowohl präskriptiv sind, weil sie sich an die Kriterien des Menschengerechten halten, als auch explikativ, »sofern sie sich im Interesse des Sachgemäßen an sozialwissenschaftlichen Erkenntnissen orientieren« (Rich 1991, S. 77). Eine Maxime des Menschengerechten könnte folglich lauten, dass Organisationen des Gesundheitswesens ihren Mitarbeitenden keine Anreize bieten sollten, die dazu führen, dass die sachgemäße Sorge für Patienten durch sachfremde, insbesondere ökonomische Interessen unterlaufen würde. Das spricht nicht gegen eine Berücksichtigung wirtschaftlicher Kriterien bei der Versorgung, aber es spricht gegen einen Vorrang von Gewinninteressen. Hier ließe sich nun argumentieren, dass ein gewisses Gewinninteresse für die Organisation unabdingbar ist, um die Investitionen, Tarifsteigerungen usw. finanzieren zu können - je hö- 
her die Investitionen, um so höher der Kapitalbedarf und so auch die Renditeerwartung der Kapitalgeber.

Die Gewinnerwartung, die im deutschen Krankenhauswesen zunächst einmal nur an Häuser in privater Trägerschaft gerichtet wird, die auch Gewinnentnahmen tätigen, entfaltet ihre Wirkung auch auf Häuser in kommunaler und freigemeinnütziger Trägerschaft, die zwar keine Kapitalgeber mit Renditen bedienen müssen, deren Leistungsfähigkeit aber an der von privaten Häuser gemessen wird. So geht von diesen gewinnorientierten Häusern und ihrer Wirtschaftsweise eine Sogwirkung auf die anderen aus, die das ganze System in Richtung Gewinninteresse dynamisiert. Diese Dynamik ist jedoch keine Sachgesetzlichkeit, die zu beachten ähnlich sachgerecht ist wie das Berücksichtigen der Gesetze der Statik beim Brückenbau. Vielmehr handelt es sich im Fall der zunehmenden Gewinnerwartungen im Gesundheitswesen um einen Sachzwang, der zwar im Prozess des Wirtschaftens begegnet, aber nicht den Charakter einer echten Sachgesetzlichkeit hat:

"Die ,Objektivität، solcher Zwänge ist jedoch prinzipiell von anderer Natur als die Objektivität der Naturgesetze, eben keine von Menschen unabhängig entstandene, sondern eine durch inn gewordene, darum geschichtliche und so auch letztlich von inm zu verantwortende Objektivität. Kurzum: der Mensch selbst setzt diese Objektivität und unterstellt sich ihr." (Rich 1991, S. 77)

Aus ethischer Perspektive ist es deshalb geboten, a) Sachgesetzlichkeiten von Sachzwängen zu unterscheiden, und b) letzteren kritisch $\mathrm{zu}$ begegnen und danach zu fragen, wie man sich von ihnen frei machen kann. Freiheit im wirtschaftlichen Handeln zu gewinnen, ist so gesehen nicht nur ein ökonomisches Interesse, sondern auch ein ethisches. Aus ethischer Perspektive besteht die Freiheit gerade darin, sogenannte Eigengesetzlichkeiten des Wirtschaftens nicht als quasi naturgesetzliche sondern als selbstgemachte Sachzwänge $\mathrm{zu}$ identifizieren und sie nicht normativ werden zu lassen, also sich ihnen zu unterwerfen. 
"Man muß aus diesen Gründen zwischen wirklichen und vermeintlichen Sachgesetzlichkeiten unterscheiden, zwischen Sachgesetzlichkeiten, die in der Rationalität des Wirtschaftens selber gründen, wie Effizienz, Wettbewerb, Planung und zwangsläufigen Folgen, die auf der strukturbestimmenden Dominanz gewisser fehlgeleiteter Grundanliegen basieren, sei es beispielsweise das extremisierte Erwerbsprinzip in der 'kapitalistischen. oder sei es das parteistaatliche Machtmaximierungsprinzip in der 'kommunistischen . Wirtschaft." (Rich 1991, S. 80)

Rich erkennt also ausdrücklich die Effizienz als innere Sachnotwendigkeit des Wirtschaftens an; sie ist demnach als sachgerecht $\mathrm{zu}$ berücksichtigen. Liegt hier nicht ein Widerspruch zu der eben vorgetragenen Argumentation vor, dass eine Berücksichtigung der Effizienz gerade kontraproduktiv sei? Gegen einen effizienten Einsatz des Personals und der Mittel ist nichts zu sagen, er ist ausdrücklich zu begrüßen, weil so eine Ressourcenverschwendung vermieden wird. ${ }^{20}$ Allerdings ist dies dann nicht mehr gegeben, wenn der effiziente Einsatz von Mittel und Personal sich nicht mehr primär an seinem Sachziel, der Versorgung von kranken, hilfebedürftigen Personen, orientiert sondern einem fremden Zweck dient: dem der maximalen Gewinnsteigerung. Sachgemäß ist der Personal- und Mitteleinsatz auch dann nicht mehr, wenn er nur einseitig der Effizienz dient und die Nachhaltigkeit beim Einsatz der Ressourcen

20 | Man kann natürlich das (bürgerliche) Axiom, unser Leben und Haushalten beruhe auf dem rationalen Umgang mit knappen Ressourcen, prinzipiell in Frage stellen, wie das etwa Georges Bataille (2001) getan hat. Dann ist nicht der effiziente Umgang mit den Ressourcen das Gebot der Stunde, sondern gerade die exzessive Verausgabung. Diese ,Antiökonomier würde den Menschen in eine zwar riskante Freiheit führen, die wenig mit den bürgerlichen Sicherheitsvorstellungen gemein habe, aber den Menschen in Einklang bringen mit der kosmischen Tendenz, alles zu verausgaben (vgl. bes. Bataille 2001, S. 289ff.). Diese Diskussion soll hier nicht geführt werden, wenngleich der Stachel der Bataille'schen Argumentation sehr viel tiefer im Fleisch der kapitalistischen Diskurse sitzt, als diese oft wahrhaben wollen. 
nicht berücksichtigt. Dies ist dann der Fall, wenn das Personal permanent über die Leistungsgrenzen hinaus gefordert wird - der Krankenstand und die Burn-out-Raten im Gesundheitswesen sind hier ein klarer Indikator (vgl. Wegner et al. 2009; Balzer 2012). Diese Situation ist unverkennbar kontraproduktiv und müsste sich allein aus ökonomischen Erwägungen schon verbieten. Dass sie dennoch auftritt - und das keineswegs sporadisch - lässt darauf schließen, dass hier andere Ursachen wirksam sind. An dieser Stelle lässt sich erkennen, dass ein starkes Gewinninteresse das Handeln der Organisationen antreibt, auch wenn sie selbst gar keine Gewinne anstreben. Es ist das, was Rich den »Erwerbswillen« nennt, der den Charakter einer Sachgesetzlichkeit annimmt und auf diese Weise einen objektiven Zwang entfaltet:

"Der Erwerbswille vermag ja ungeheure Produktivkräfte freizulegen [...]. Wird nun dieses Grundinteresse zum beherrschenden Strukturprinzip, dann entstehen wieder Notwendigkeiten oder Zwänge, die aber nicht mit der rationalen Struktur des Wirtschaftens selbst zusammenhängen, sondern mit einer bestimmten, eben durch die Dominanz des Erwerbsmotivs geprägten Gestalt oder Ordnung der Wirtschaft." (Rich 1991, S. 79).

Hier besteht die ethische Aufgabe vorrangig darin, die Fehlsteuerung allein unter Effizienzgesichtspunkten als ein selbstgemachtes Problem zu erkennen und ihm zu begegnen. Es ist nicht die Wirtschaft selbst, die uns dazu zwingt, Mitarbeitende über die Grenzen ihrer physischen und psychischen Leistungsfähigkeit hinaus zu fordern. Es ist auch nicht ein Sachzwang der Ökonomie per se, dass Patienten Leistungen erhalten, die sie aus medizinischer oder pflegerischer Sicht gar nicht brauchen, während umgekehrt anderen Patienten Leistungen vorenthalten werden, weil die Leistungserbringung sich für die Organisation nicht lohnt. Es sind dies vielmehr Effekte, die sich einer vorrangig auf Effizienz ausgerichteten, an der Nachhaltigkeit jedoch wenig interessierten Wirtschaftsweise >verdankt<. Diese als selbstgemachten Sachzwang zu identifizieren 
und Freiheit für Alternativen zu gewinnen, ist die vornehmste Aufgabe der Ethik an dieser Stelle.

\section{LiteratuR}

Aristoteles (1995): Nikomachische Ethik. In: Aristoteles. Philosophische Schriften, in sechs Bänden, Band 3. Hamburg: Meiner.

Balzer, R. (2012): Krankenhäuser im Burnout - Herausforderungen an die Unternehmensführung in diakonischen Unternehmen. In: Stockmaier, J.; Giebel, A.; Lubatsch, H. (Hg.): Geistesgegenwärtig pflegen. Existenzielle Kommunikation und spirituelle Ressourcen im Beruf. Band 1: Grundlegungen und Werkstattberichte. Neukirchen-Vluyn: Neukirchener Verlag, 31-40.

Bataille, G. (2001): Die Aufhebung der Ökonomie, 3. erw. Aufl. München: Matthes \& Seitz.

Bohsem, G. (2009): Systematisch korrupt. Medizinisch-Industrieller Komplex. In: Süddeutsche Zeitung Online 03.09.2009, zu finden unter: www.sueddeutsche.de/geld/medizinisch-industriellerkomplex-systematisch-korrupt-1.37323, Zugriff: 14.12.2012.

Buestrich, M.; Wohlfahrt, N. (2008): Die Ökonomisierung der Sozialen Arbeit. Aus Politik und Zeitgeschichte, H. 12-13: 17-24.

Braun, B.; Buhr, P.; Klinke, S.; Müller, R.; Rosenbrock, R. (2010): Pauschalpatienten, Kurzlieger und Draufzahler - Auswirkungen der DRGs auf Versorgungsqualität und Arbeitsbedingungen im Krankenhaus. Bern: Huber.

Brodbeck, K.H. (1998): Die fragwürdigen Grundlagen der Ökonomie. Eine philosophische Kritik der modernen Wirtschaftswissenschaften. Darmstadt: Wissenschaftliche Buchgesellschaft.

Deutsches Institut für Pflegeforschung (2012): Pflegethermometer 2012. Eine bundesweite Befragung von Leitungskräften zur Situation der Pflege und Patientenversorgung auf Intensivstationen im Krankenhaus. Köln: dip. 
Deutschmann, C. (2009): Geld und kapitalistische Dynamik. In: Nissen, S.; Vobruba, G. (Hg.): Die Ökonomie der Gesellschaft. Wiesbaden: Verlag für Sozialwissenschaften, 57-71.

DGB-Index »Gute Arbeit« für die Bereiche Krankenpflege und Altenpflege, zu finden unter: www.verdi-gute-arbeit.de/upload/ m49d5c6613279f_verweis1.pdf, Zugriff: 10.12. 2012 sowie zu finden unter: www.verdi-gute-arbeit.de/upload/m49d5c6613279f_ verweis2.pdf, Zugriff: 10. 12. 2012.

Dietz, A. (2011): Gerechte Gesundheitsreform? Ressourcenvergabe in der Medizin aus ethischer Perspektive. Frankfurt a.M./New York: Campus.

Dietz, A.M.; Charbonnier, R.; Manzeschke, A. (Hg.) (2007): Aktiengesellschaft Krankenhaus. Bestimmen ökonomische Ziele medizinisches Handeln? (Schriften zur Bayreuther Gesundheitsökonomie Bd. 6). Bayreuth: P.C.O.-Verlag.

Freidson, E. (1979): Der Ärztestand. Berufs- und wissenschaftssoziologische Durchleuchtung einer Profession. Stuttgart: Enke.

Faller, H.; Grefe, C. (2012): Krankenhäuser Geld oder Leben. Die Zeit Nr. 39 vom 20. 9. 2012.

Heubel, F.; Kettner, M.; Manzeschke, A. (Hg.) (2010): Die Privatisierung von Krankenhäusern. Ethische Perspektiven. Wiesbaden: VS-Verlag.

Heubel, F. (2010): Therapeutische Interaktion und Funktionslogik des Marktes. In: Hebel. F.; Kettner, M.; Manzeschke, A. (Hg.): Die Privatisierung von Krankenhäusern. Ethische Perspektiven. Wiesbaden: VS-Verlag, 165-194.

Hug, S. (2009): Handeln in Organisationen - Zwischen Struktur und Kultur. In: Baumann-Hölzle, R.; Arn, C. (Hg.): Handbuch Ethik im Gesundheitswesen, Bd. 3: Ethiktransfer in Organisationen. Basel: Schwabe, 17-30.

Huster, S. (2011): Soziale Gesundheitsgerechtigkeit. Sparen, umverteilen, vorsorgen? Berlin: Wagenbach.

Isfort, M.; Weidner, F.; Pflege-Thermometer (2007): Eine bundesweite repräsentative Befragung zur Situation und zum Leistungsspektrum des Pflegepersonals sowie zur Patientensicherheit im Krankenhaus, 
hg. von Deutsches Institut für angewandte Pflegeforschung e.V. Köln: dip.

Isfort, M.; Weidner, F.; (2010): Pflege-Thermometer 2009. Eine bundesweite Befragung zur Situation der Pflege und Patientenversorgung im Krankenhaus, hg. von Deutsches Institut für angewandte Pflegeforschung e.V. Köln: dip.

Jäger, W.; Coffin, A.R. (2011): Die Moral der Organisation. Beobachtungen in der Entscheidungsgesellschaft und Anschlussüberlegungen $z$ u einer Theorie der Interaktionssysteme. Wiesbaden: VS-Verlag.

Johnson, L.; Sattler, D. (2005): Studie zur Berufssituation von Ärzten. Im Auftrag der Kassenärztlichen Vereinigung Schleswig-Holstein, Bad Segeberg.

Kettner, M.; Koslowski, P. (Hg.) (2011): Ökonomisierung und Kommerzialisierung der Gesellschaft. Wirtschaftsphilosophische Unterscheidungen. München: Wilhelm Fink.

Kiesel, J.; Broding, H.C.; Lederer, P.; Kötter, R.; Schmid, K.; Drexler, H. (2010): Arbeitszufriedenheit, selbsteingeschätzter Gesundheitszustand und Beanspruchungen von Mitarbeitern eines Universitätsklinikums im Vergleich zu anderen Betrieben. Unterschiede in der Bewertung von Klinikärzten/innen und Pfleger/ innen. Arbeitsmedizin, Sozialmedizin, Umweltmedizin o7: 416422.

Kölking, H. (2007): Entwicklung der Gesundheitswirtschaft in Deutschland. In: Kölking, H. (Hg.): DRG und Strukturwandel in der Gesundheitswirtschaft. Stuttgart: Kohlhammer, 19-31.

Krobath, T.; Heller, A. (Hg.) (2010): Ethik organisieren. Handbuch der Organisationsethik. Freiburg/Brsg.: Lambertus.

Krainer, L.; Heintel, P. (2010): Prozessethik. Zur Organisation ethischer Entscheidungsprozesse. Wiesbaden: Springer VS.

Kumbruck, C.; Rumpf, M.; Senghaas-Knobloch, E. (2010): Unsichtbare Pflegearbeit. Fürsorgliche Praxis auf der Suche nach Anerkennung. Münster: Lit.

Manzeschke, A. (2011): Ökonomisierung - Zur Erklärungskraft und Klärungsbedürftigkeit eines Begriffs. In: Kettner, M. und Koslowski, P. (Hg.): Ökonomisierung und Kommerzialisierung der Ge- 
sellschaft. Wirtschaftsphilosophische Unterscheidungen. München: Wilhelm Fink, 67-93.

Manzeschke, A. (2010a): Transformation der Pflege - Ethische Aspekte eines subtilen und zugleich offenkundigen Wandels. In: Kreutzer, S. (Hg.): Transformation pflegerischen Handelns. Institutionelle Kontexte und soziale Praxis vom 19. bis 21. Jahrhundert. (= Schriftenreihe zur Pflegewissenschaft und Pflegebildung Bd. 5). Göttingen: Vandenhoeck \& Ruprecht unipress, 175-193.

Manzeschke, A. (2010b): Eigeninteresse und Verantwortung - Zu einer notwendigen Revision des homo-oeconomicus-Modells. In: Manzeschke, A. (Hg.): Sei ökonomisch. Prägende Menschenbilder zwischen Modellbildung und Wirkmächtigkeit. Münster: Lit, 131-162.

Manzeschke, A.; Brink, A. (2010): Versprechen, Vertrag und Supererogation in Nonprofit-Organisationen. In: Theuvsen, L.; Schauer, R.; Gmür, M. (Hg.): Stakeholdermanagement in Non-Profit-Organisationen. Linz: Trauner, 125-141.

Marx, K. (1972): Das Kapital. Kritik der politischen Ökonomie. Berlin: Dietz (=Marx-Engels-Werke Band 23).

Nefiodow, L.A. (2000): Der sechste Kondratieff. Wege zur Produktivität und Vollbeschäftigung im Zeitalter der Information. 4. Aufl. Sankt Augustin: Rhein-Sieg-Verlag.

Oberender, P.; Hebborn, A.; Zerth, J. (2010): Wachstumsmarkt Gesundheit. 3. Aufl. Stuttgart: Lucius \& Lucius.

Polanyi, K. (1978): The Great Transformation. Politische und ökonomische Ursprünge von Gesellschaften und Wirtschaftssystemen. Frankfurt a.M.: Suhrkamp.

Rich, A. (1991): Wirtschaftsethik. Band I: Grundlagen in theologischer Perspektive. 4. Aufl. Gütersloh: Gütersloher Verlagshaus.

Roubini, N.; Mihm, S. (2010): Das Ende der Weltwirtschaft und ihre Zukunft. Crisis Economics. Frankfurt a.M.: Campus.

Schimank, U.; Volkmann, U. (2008): Ökonomisierung der Gesellschaft. In: Maurer, A. (Hg.): Handbuch der Wirtschaftssoziologie. Wiesbaden: VS, 382-393. 
Transparency International (2006): Jahrbuch Korruption 2006: Schwerpunkt: Gesundheitswesen: Schwerpunkt: Korruption im Gesundheitssektor. Berlin: Parthas.

Schuh, F. (2007): Hilfe! Ein Versuch zur Güte. Graz: Styria.

Sedlácek, T. (2012): Die Ökonomie von Gut und Böse. Darmstadt: Büchergilde Gutenberg.

Ulrich, P. (2008): Integrative Wirtschaftsethik. Grundlagen einer lebensdienlichen Ökonomie. 4. Aufl. Bern/Stuttgart/Wien: Haupt.

Ulrich, P. (2010): Zivilisierte Marktwirtschaft. Eine wirtschaftsethische Orientierung. Bern/Stuttgart/Wien: Haupt.

Vogl, J. (2010): Das Gespenst des Kapitals. Zürich: diaphanes.

Wegner, R.; Kostova, P.; Poschadel, B.; Manuwald, U.; Baur, X. (2009): Belastung und Beanspruchung von Krankenhausärzten/-innen. Ergebnisse einer aktuellen Erhebung. Arbeitsmedizin, Sozialmedizin, Umweltmedizin 44(7):389-399.

Wünsch, G. (1927): Evangelische Wirtschaftsethik. Tübingen: J. C. B. Mohr. 


\title{
Autorenverzeichnis
}

\author{
Kathrin Dengler \\ Kathrin Dengler ist Mitarbeiterin am Institut für Wirtschaftswis- \\ senschaften der Universität Ulm. Forschungsschwerpunkte: Ver- \\ haltensökonomie und experimentelle Ökonomie sowie ethische \\ Komponenten in der Entscheidungsfindung. Zuvor war sie Mit- \\ arbeiterin am Institut für Geschichte, Theorie und Ethik der Medi- \\ zin der Universität Ulm.
}

\section{Heiner Fangerau}

Prof. Dr. Heiner Fangerau ist Direktor des Institutes für Geschichte, Theorie und Ethik der Medizin der Universität Ulm. Forschungsschwerpunkte: Geschichte und Ethik der Biomedizin, Geschichte und Ethik der Psychiatrie, Entwicklung der medizinischen Diagnostik, Evolution medizinischen Wissens und Handelns.

\section{Günter Fröhlich}

PD Dr. Günter Fröhlich war von 2009 bis 2012 Gastprofessor für Philosophie am Humboldt-Studienzentrum der Universität Ulm und geht jetzt wieder seinen Lehrverpflichtungen in Regensburg nach. Forschungsschwerpunkte: Praktische Philosophie (systematisch und historisch), klinische Ethik, Philosophische Anthropologie, philosophische Methoden.

\section{Sebastian Kessler}

Sebastian Kessler ist Wissenschaftlicher Mitarbeiter am Institut für Geschichte, Theorie und Ethik der Medizin der Universität Ulm. 
Forschungsschwerpunkte: Geschichte und Ethik sozialer Ungleichheit, Psychiatriegeschichte, Global Health.

\section{Reinhold Kilian}

Prof. Dr. Reinhold Kilian ist Leiter der Sektion Gesundheitsökonomie und Versorgungsforschung der Abteilung Psychiatrie und Psychotherapie II der Universtität Ulm. Forschungsschwerpunkte: Soziale und ökonomische Grundlagen psychiatrischer Versorgung, gesundheitsökonomische Evaluation, Lebensqualitätsforschung, Empowerment, Methodenentwicklung.

\section{Christian Lenk}

PD Dr. Christian Lenk ist Geschäftsführer der Ethikkommission der Universität Ulm. Forschungsschwerpunkte: Enhancement, Ethikkommissionen, Forschungsethik, Gerechtigkeit, Technikfolgenabschätzung, Wissenschaftstheorie.

\section{Arne Manzeschke}

PD Dr. theol. habil. Arne Manzeschke ist Leiter der Fachstelle für Ethik und Anthropologie im Gesundheitswesen am Institut Technik - Theologie - Naturwissenschaften (TTN) an der Ludwig-Maximilians-Universität München. Forschungsschwerpunkte: Wirtschafts-, Technik- und Bioethik, Ethik und Anthropologie, Mensch-Technik-Interaktion.

\section{Walter Pfannkuche}

Prof. Dr. Walter Pfannkuche lehrt Praktische Philosophie an der Universität Kassel. Er leitet ein Forschungsprojekt zur Ethik der Globalisierung. 


\section{Register}

A

Absicherungsintensität $\quad 120 \mathrm{ff}$.

Aggregationsprinzip 117, 134f., Allokationskriterien 86

Anerkennung 12, 15, 18, 63, $66,127,139,143 f f ., 177,232$

Anreizsysteme 231, 235

Anspruchsniveau 183, 186, 189

Armut 97, 124, 139, 142, 157, 163

Arzt-Patient-Beziehung, Arzt-

Patient-Verhältnis 68

Ästhetik 198

\section{B}

Begründungstypen 19, 195,

202, 204, 213

Behandlungen 59, 82ff., 98f., 102ff., 126, 188

Medizinisch indizierte

Behandlungen 84

Behinderung, Grad der

Behinderung 14, 43, 181

Bewertungsverfahren 17 ,

$27 \mathrm{ff} ., 45,51,55,62 \mathrm{ff} ., 67 f .$,

200, 202
Bruttoinlandsprodukt, BIP 27,

$$
\text { 44, } 89 f \text {. }
$$

Budget, Globalbudget $\quad 66 f$., 69, 99f., 104, 113, 125, 132f., 217, 232

C

Chemotherapie 181

D

Degeneration $15 \circ$.

Deontologie 19, 80, 207, 209f.

Depravation 15of., 154

disability-adjusted life years,

$$
\text { DALY } 40,44,46,54,129
$$

E

Effektivität $\quad 32,37,70,90$,

$$
104 \text { ff., } 234
$$

Effizienz 19, 32, 53, 88, 95, 104, 113, 223, 228f., 231, 234ff., 238, 24 of.

Egalitaristen 117

Einkommenspreizung 124

Entscheidungsfindung 42,

69, 71 
Einzelfallentscheidung 199f., 206

Entscheidungspräferenzen 178 Epidemiologie 18, 139f., 143, $147,158,163$

Erbgut 145

Ethik

Antike 97

Christliche 97

deontologisch 19, 202,

204, 206, 210

Ethiktypen 214

Konsequentialistische 19,

68, 202f.

Maximenethik 207

Tugendbasierte 202

Eudaimonia 211ff. eudämonisch 176f., 180, $182 f$.

EuroQol 180, 183

Euthanasie $152,155,160$

Evaluationsverfahren 17, 28f., 6of., 66, 69, 71

gesundheitsökonomische

Evaluationsverfahren 66

Evidenz, wissenschaftliche 230

F

Fehlallokationen 9of., 106, 231

Fehlsteuerung 202, 241

Folgenabschätzungen 204, 248

Fortpflanzung 146, 149, 151f., 154ff., 161
Freiheit 15, 118, 153, 175f., 209, 239f., 242

Freude 176, 212

Fürsorge 113ff., 118, 121f., 124, 133f., 142, 153, 156, 196, 214 Soziale 153

G

Gebrauchswert 197

Geldeinheiten 36f., 41, 46

Gemeinwohl 97, 206, 209, 215

Gerechtigkeit

Theorie der Gerechtigkeit 96,198

Gesetze 8, 100, 126, 196, 206f., 217, 237, 239

Gesundheitssystem 27f., 34, $36,38,52,65,83,85,89$ ff., 101, 105, 107, 122, 195, 204, 214f., 228, 230

Gesundheitszustände 11f., 41f., 55,183

Gewinninteresse 238f., 241

Gewinnsteigerung 240

Glück $6_{4}, 67,81,87$, 117ff., $176,185,205,211$

Gossensche Gesetze 10of.

Grenznutzen 99ff., 229

Gut, Güter

Güterknappheit 92f., 96f. kontingent knappe Güter 94 kontingent nicht knappe Güter 94, 100 
Lebensnotwendige Güter
80
notwendig knappe Güter
94
notwendig nicht knappe
Güter 94

Gutes Leben 176

$\mathbf{H}$

Handlungsoffenheit 200

Handlungsalternativen 202

Handlungsfolgen 209, 214

Handlungsgrundsätze 210

Handlungsprinzipien 210

Handlungsregeln 200, 210f.

Health Utilities Index, HUI

88

Healthy Year Equivalents, HYE 39ff., 46, 54, 6off., 129

Hedonistisch 175f., 178, 180, $182 f$.

Homöostase $186 f f$.

Lebensqualitätshomöostase 186

Hygiene, Soziale 147f., 157

\section{I}

Individualisierung $121 f$.

Ineffizienzen 113, 231, 234

Interessen 42f., 54, 68, 116ff., $123,156,161,182,196,205$, 208ff., 214, 232ff., 238

Internationale Klassifikation

Funktionsfähigkeit,
Behinderung und

Gesundheit, ICF 181

K

Kantische Moral $207 f$.

Kaufentscheidung 85

Knappheit 84, 92ff., 101, $107 f$.

Kollektives Gut 123

Konkurrenzfähigkeit $\quad 228$

Körperfunktionen 183

Korruption 231f.

Kosten

Direkte Kosten 30 Indirekte Kosten 30

Kostenbegriff 30

Kostenanalyse $34,45,54$

Kostenausweitung 230

Kosten-Effektivitäts-Analyse 37

Kosten-Kosten-Analyse 33ff., 46,54

Kosten-Nutzen-Analyse (cost-benefit analysis, benefit-cost analysis) 36f., 46, 54

Kosten-Nutzwert-Analyse 17 , 32, 38f., 41, 43, 54

Kostenvergleichsrechnung 35 Krankenversicherung 8,83f., 118f., 121, 127, 130, 132, 136 .

228

Gesetzliche, GKV 83,103, 127

Private, PKV 83 
L

Lebenserwartung 31,38ff., 44, 46, 61, 89ff.

Lebensqualität

Beurteilung der

Lebensqualität 176

Lebensqualitätsmessung 19, 171, 178f., 182f., 189

Lebensqualitätsforschung $177 f$., 190

Lebensspanne, verbleibende

Lebensspanne 86

Lebensverlängerung 83,199

Leid 15f., 47, 83, 135, 151, 174ff., 217

Leistungserbringer 29

Lust, Verzicht auf Lust 176 , 205

M

Mehrheitsentscheid 206

Menschenwürde 61,107,206, 208

Messtheorie 175,180

Mittel

Mittelallokation 88

Mitteleinsatz 28, 33, 35,

$224,234,240$

Moralsysteme 215

Morbidität 105f, 139, 141, 148, 155, 159, 227

Mortalität 106, 139, 141,148,

155,159
N

Naturgesetz 232, 239

Nutzen

Individueller Nutzen 67 ,

82

Ökonomischer Nutzen 83

Nutzentheorie, nutzentheoretisch 178 , 180,183

Nützlichkeit,

Nützlichkeitsgründe 81 ,

197, 206, 225

Nutzwert 17, 29, 31f., 38ff., 47,

$51,54 f ., 57,179,183,198$

Nutzwertverluste 183

O

Ökonomisierung 107, 228f., 232, $234 \mathrm{f}$.

Ökonomisierung des

Gesundheitssystems 228

Outcome 3of., 41f.

P

Palliativmedizin $\quad 175,181$

Partizipation, soziale 129,146 , 161,181

Patient

Wünsche des Patienten

196

Patientenversorgung 215

Personaleinsatz 234

Persönlichkeit 145,188

Pflege 29, 174, 232, 234ff. 
Pluralismus $\quad 65,107,119,122 f ., \quad S$

177

Präferenzen 16, 19, 41ff., 45f.,

$54,57,65,79,121,123,131$, $178,183,197$

Prinzipien 18, 78, 81, 103f., $107,116,178,199$, 205ff., 211, $213 f$.

Priorisierung 52,57, 62, 69f., 81, 102f., 105f., 108, 127, 134, 172

Produktivität $30,80,95,172$

Prozessorganisation 234

Q

Quality-of-Wellbeing-Skala, QWB 88

QALY 39ff., 54ff., 58ff., 67, 82, 86ff., 101, 103, 105, 108, 114, $129,134,183$

\section{$\mathbf{R}$}

Rasse, Rassentheorie 142,149 , $153 \mathrm{ff} ., 161$

Rationalisierung 52, 113, 177

Rationierung $18,52 f ., 56,113$, 127, $132 \mathrm{ff}$.

Rechtssicherheit 201

Regeln 13, 28, 78, 148, 207, 209f.

Response shift 60

Ressourcen

Ressourcenknappheit 84 , 97

Ressourcenverzehr 29

Retorsionsproblem 202
Sachgesetz/

Sachgesetzlichkeiten 232, 239 ff.

Sachlogik 228

Sachzwang 232, 239, 241

Satisfaction with Life Scale 178

Save Young Lives Equivalents, SAVE $40,42 f ., 46,54,58$, 60

Schmerz 13, 30, 38, 84, 175f., 212

Selbstbestimmung 122f., 125, 127f., 132, 196, 204, 206

Selbstkonstitution $144 \mathrm{f}$.

$\mathrm{SF}_{3} 6180$

Sorge 149, 227, 231, 238

Sozialdarwinismus, sozialdarwinistisch 151, 153, 155f., 160

Sozialepidemiologie 18, 139f., $143,147,158,163$

Sozialmedizin 140

Sphären 77ff., 107, 226

Spieltheorie 178

Standard Gamble (SG)

Verfahren 42

Subjekte 92f., 117, 122,146, 197,200

\section{$\mathbf{T}$}

Tapferkeit 176

Tausch 6o, 65, 81, 97, 197f., 223,231

Teleologisch 176, 236 
Transzendentales Gut 120

Tugend 57, 202, 204, 211ff., 218

Tugendethik 19, 211ff.

U

Umweltbedingungen 147ff., 181,184

Unparteilichkeit, unparteiisch 115ff., 121ff., 136

Utilitarismus/Utilitaristische

Ethik 19, 67, 82, 86f., 117, 205f., $208 \mathrm{ff}$., $213 \mathrm{f}$.

Regelutilitarismus 209

V

Vererbungshygieniker 155,161

Vermögensverteilung 124

Versicherungspflicht 99

Versicherungssystem 85,106, 228

Versorgungssystem 28, 113

Volkskörper $147,152,157,159$, $161 f$.

Volkswirtschaft $27,29,34,45$, 82,228

W

Wachstumsgrenze 214

Wahrheit 154, 176, 199, 223

Wert

Monetäre Werte 204

Wertbegriff 198

Werthaltung 16,19, 195,

199, $201 f f$.
Werthaltungstypen 202

Wertphilosophie 198

Wertprädikate 199

Wertverteilungen 202

Wertvorstellungen 199

Wertzuordnungen 202

Wertschätzung 42, 45, 143,

145 f., 150

Soziale $145 \mathrm{f}$.

Wertschöpfung 28, 230

WHO 10, 44, 179ff.

Wirksamkeit 31f., 37ff., 45f., 51,

$54,103,183,186,188 \mathrm{f}$.

klinische Wirksamkeit 32

Wohlbefinden

Subjektives Wohlbefinden

$185 f$.

Wohl des Patienten 196

Wohlergehen $31,68,107,152$,

201, 212

Wohltun 31

World Health Organization

Quality of Life, WHOQOL 179ff.

\section{$\mathrm{Z}$}

Zuzahlungsregeln 85

Zwangsmaßnahme 18, 148, 152f., $155,160 f$.

Zwangssterilisation $\quad 152,161$ 


\section{KörperKulturen}

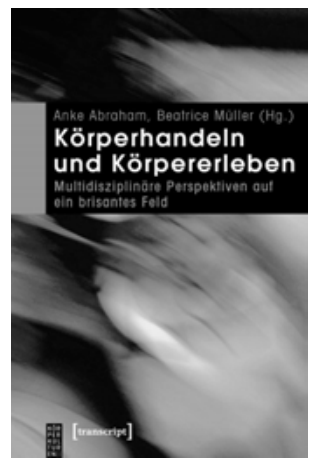

Anke Abraham, Beatrice Müller (Hg.)

Körperhandeln und Körpererleben

Multidisziplinäre Perspektiven

auf ein brisantes Feld

2010, 394 Seiten, kart., zahlr. z.T. farb. Abb., $32,80 €$, ISBN 978-3-8376-I227-I

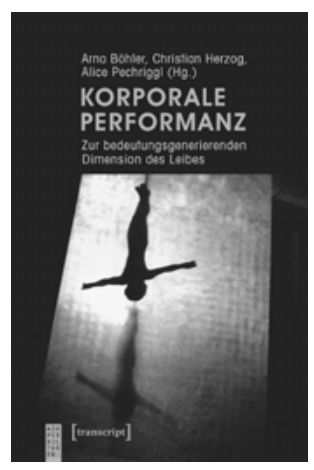

Arno Böhler, Christian Herzog, Alice Pechriggl (Hg.)

Korporale Performanz

Zur bedeutungsgenerierenden Dimension des Leibes

Oktober 2013, 274 Seiten, kart., 29,99€, ISBN 978-3-8376-2477-9

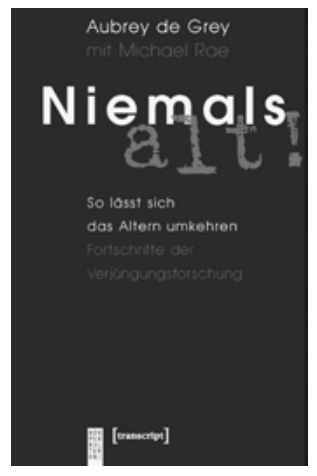

Aubrey de Grey, Michael Rae

Niemals alt!

So lässt sich das Altern umkehren.

Fortschritte der Verjüngungsforschung

20I0, 396 Seiten, kart., 2I, $80 €$,

ISBN 978-3-8376-I336-0

Leseproben, weitere Informationen und Bestellmöglichkeiten finden Sie unter www.transcript-verlag.de 


\section{KörperKulturen}

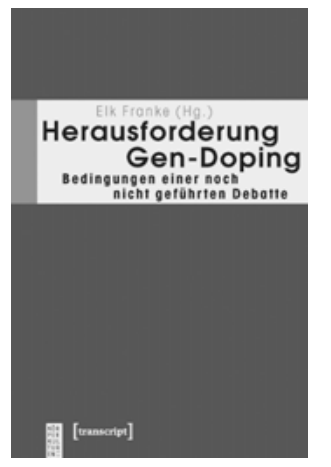

ElK Franke (Hg.)

Herausforderung Gen-Doping

Bedingungen einer noch nicht geführten Debatte

Dezember 20I3, ca. 270 Seiten, kart., I9,80€,

ISBN 978-3-8376-I380-3

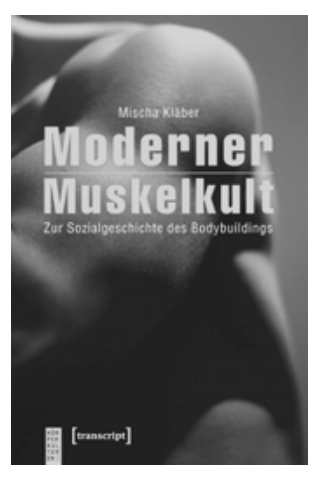

MisCha KLÄBER

Moderner Muskelkult

Zur Sozialgeschichte des Bodybuildings

April 2013, 276 Seiten, kart., $28,80 €$, ISBN $978-3-8376-2376-5$

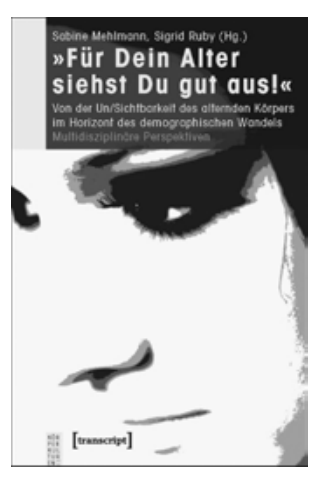

Sabine Mehlmann, Sigrid Ruby (Hg.)

»Für Dein Alter siehst Du gut aus!«

Von der Un/Sichtbarkeit des alternden Körpers im Horizont des demographischen Wandels.

Multidisziplinäre Perspektiven

20Io, 278 Seiten, kart., zahlr. Abb., 26,80€,

ISBN 978-3-8376-I32I-6

Leseproben, weitere Informationen und Bestellmöglichkeiten finden Sie unter www.transcript-verlag.de 


\section{KörperKulturen}

Karl-Heinrich Bette

Sportsoziologische Aufklärung

Studien zum Sport der modernen

Gesellschaft

20II, 260 Seiten, kart., $28,80 €$,

ISBN 978-3-8376-I725-2

Karl-Heinrich Bette,

Felix Kühnle, Ansgar Thiel

Dopingprävention

Eine soziologische Expertise

20I2, 232 Seiten, kart., zahlr. Abb., 25,80€, ISBN 978-3-8376-2042-9

Franz Bockrath (HG.)

Anthropotechniken im Sport

Lebenssteigerung durch

Leistungsoptimierung?

20II, 264 Seiten, kart., zahlr. Abb., 27,80€, ISBN 978-3-8376-1868-6

\section{Julia DiekäMPER}

Reproduziertes Leben

Biomacht in Zeiten der

Präimplantationsdiagnostik

20II, 4I6 Seiten, kart., 35,80€,

ISBN 978-3-8376-I8II-2

Karen Ellwanger,

Heidi Helmhold,

Traute Helmers,

Barbara Schrödl (Hg.)

Das »letzte Hemd «

Zur Konstruktion von Tod

und Geschlecht in der

materiellen und visuellen Kultur

20Io, 360 Seiten, kart.,

zahlr. z.T. farb. Abb., $35,80 €$,

ISBN 978-3-8376-1299-8
Nicholas Eschenbruch,

Dagmar HÄNEL,

Alois Unterkircher (Hg.)

Medikale Räume

Zur Interdependenz von Raum,

Körper, Krankheit und Gesundheit

20I0, 254 Seiten, kart., zahlr. Abb., 26,80 €,

ISBN 978-3-8376-I379-7

Nino FERRIN

Selbstkultur und mediale Körper

Zur Pädagogik und Anthropologie neuer Medienpraxen

Juli 20I3, 246 Seiten, kart., 29,80€, ISBN 978-3-8376-2505-9

\section{Robert GugutZer}

Verkörperungen des Sozialen

Neophänomenologische Grundlagen und soziologische Analysen

20I2, 256 Seiten, kart., 28,80 €,

ISBN 978-3-8376-I908-9

Charlotte Ullrich

Medikalisierte Hoffnung?

Eine ethnographische Studie zur reproduktionsmedizinischen Praxis 2012, 356 Seiten, kart., 33,80€, ISBN 978-3-8376-2048-I

HeInZ-JÜrgen Voss

Making Sex Revisited

Dekonstruktion des Geschlechts aus biologisch-medizinischer Perspektive (3., unveränderte Auflage 20II)

20Iо, 466 Seiten, kart., $34,80 €$,

ISBN 978-3-8376-I329-2

Karen Wagels

Geschlecht als Artefakt

Regulierungsweisen in

Erwerbsarbeitskontexten

Januar 2013, 276 Seiten, kart., 29,80 €, ISBN 978-3-8376-2226-3 
